\title{
Development of Real-time Optimal Control Strategy of Hybrid Transit Bus Based on Predicted Driving Pattern
}

Wei Qi

Follow this and additional works at: https://researchrepository.wvu.edu/etd

\section{Recommended Citation}

Qi, Wei, "Development of Real-time Optimal Control Strategy of Hybrid Transit Bus Based on Predicted Driving Pattern" (2016). Graduate Theses, Dissertations, and Problem Reports. 6459.

https://researchrepository.wvu.edu/etd/6459

This Dissertation is protected by copyright and/or related rights. It has been brought to you by the The Research Repository @ WVU with permission from the rights-holder(s). You are free to use this Dissertation in any way that is permitted by the copyright and related rights legislation that applies to your use. For other uses you must obtain permission from the rights-holder(s) directly, unless additional rights are indicated by a Creative Commons license in the record and/ or on the work itself. This Dissertation has been accepted for inclusion in WVU Graduate Theses, Dissertations, and Problem Reports collection by an authorized administrator of The Research Repository @ WVU.

For more information, please contact researchrepository@mail.wvu.edu. 


\title{
Development of Real-time Optimal Control Strategy of Hybrid Transit Bus Based on Predicted Driving Pattern
}

\author{
Wei Qi \\ Dissertation submitted to the \\ Benjamin M. Statler College of Engineering and Mineral Resources \\ at West Virginia University \\ in partial fulfillment of the requirements for the degree of \\ Doctor of Philosophy In \\ Mechanical Engineering \\ Hailin Li, Ph.D., Chair \\ Nigel N. Clark, Ph.D. \\ Yu Gu, Ph.D. \\ Natalia A. Schmid, Ph.D. \\ W. Scott Wayne, Ph.D. \\ Department of Mechanical and Aerospace Engineering
}

Morgantown, West Virginia

2016

Keywords: Hybrid Transit Bus, Control Strategy, Dynamic Programming, Equivalent Consumption Minimization Strategy, Adaptive ECMS Copyright 2016 Wei Qi 


\section{Abstract \\ Development of Real-time Optimal Control Strategy of Hybrid Transit Bus Based on Predicted Driving Pattern \\ Wei Qi}

The control strategy of a hybrid electric vehicle (HEV) has been an active research area in the past decades. The main goal of the optimal control strategy is to maximize the fuel economy and minimize exhaust emissions while also satisfying the expected vehicle performance. Dynamic programming (DP) is an algorithm capable of finding the global optimal solution of HEV operation. However, DP cannot be used as a real-time control approach as it requires pre-known driving information. The equivalent consumption minimization strategy (ECMS) is a real-time control approach, but it always results in local optima due to the non-convex cost function.

In my research, a ECMS with DP combined model (ECMSwDP) was proposed, which was a compromise between optimality and real-time capability. In this approach, the optimal equivalent factor $(\lambda)$ for a real-time ECMS controller can be derived using ECMSwDP for a given driving condition. The optimal $\lambda$ obtained using ECMSwDP was further processed to derive the $\lambda$ map, which was a function of vehicle operation and driving information. Six $\lambda$ maps were generated corresponding to the developed representative driving patterns. At each distance segment of a drive cycle, the suitable $\lambda$ value is available from one of the six $\lambda$ maps based on the identified driving pattern and current vehicle operation.

An adaptive ECMS (A-ECMS) model with a driving pattern identification model is developed to achieve the real-time optimal control for a HEV. A-ECMS was capable of controlling the ratio of power provided by the ICE and battery of a hybrid vehicle by selecting the $\lambda$ based on the identified $\lambda$ map. The effect on fuel consumption of the control strategies developed using the rule-based controller, ECMSwDP, A-ECMS, and DP was simulated using the parallel hybrid bus model 
developed in this research. The control strategies developed using A-ECMS are able to significantly improve the fuel economy while maintaining the battery charge sustainability. The corrected fuel economy observed with A-ECMS with a penalty function and the average $\lambda$ of RDPs was $5.55 \%, 13.67 \%$, and $19.19 \%$ gap to that observed with DP when operated over the Beijing cycle, WVU-CSI cycle, and the actual transit bus route, respectively. The corrected fuel economy observed with A-ECMS with $\lambda$ maps of the RDPs was $4.83 \%, 10.61 \%$, and $14.33 \%$ gap to that observed with DP when operated on the Beijing cycle, WVU-CSI cycle, and actual transit bus route, respectively.

The simulation results demonstrated that the proposed A-ECMS approaches have the capability to achieve real time suboptimal control of a HEV while maintaining the charge sustainability of the battery. 


\section{Acknowledgments}

I would like to express my deep gratitude to my advisor, Dr. Hailin Li. His guidance, patience, and encouragement were invaluable through my doctoral study at West Virginia University. His passion for research will affect me during my future academic life. Thank him for his advice and help in the past several years. I really wouldn't be here without him, thanks so much for everything he has done for me.

I would also like to extend my thanks to my advisory committee members, Dr. Nigel N. Clark, Dr. Yu Gu, Dr. Natalia A. Schmid, and Dr. W. Scott Wayne for their important suggestion and kindly help during the course of this research work.

I appreciate my colleagues and friends who have helped and collaborated with me: Jingang Liu, Xinyou Lin, Shiyu Liu, Pingen Chen, Yu Li, Lijuan Wang, Ahmed AI-Samari, Zhenhua Zhu, Jairo A. Sandoval León, Clay Bell and Garrett Rinker. Without their help, it is impossible for me to have a smooth and joyful life in WVU. Thank all my friends, here and in China, for your constant support and care. Special thanks to Dr. Rui Wang (North Carolina State University) for patiently addressing the many questions I had about algorithm. Thanks to West Virginia University, MAE department, and CAFEE for their financial support.

I offer my deepest thanks and gratitude to my family members, especially parents and parents-inlaw for their love and support. Lastly, I would like to express my thanks to my dearest wife, Yuejuan Li, my daughter, Ivy Qi and my son, Leo Qi. They are the greatest source of strength, inspiration and happiness. Without them, I cannot imagine my life. I am truly thankful to everyone who has contributed to make this research possible. 


\section{Table of Contents}

$\begin{array}{lr}\text { Abstract } & \text { ii } \\ \text { Acknowledgments } & \text { iv } \\ \text { Table of Contents } & \text { v } \\ \text { List of Figures } & \text { xvi } \\ \text { List of Tables } & \text { xviii } \\ \text { Nomenclature } & \text { 1 } \\ 1 \quad \text { Introduction } & \end{array}$

1.1 Background __ 1

1.2 Motivation _ 3

1.3 Objectives of Thesis__ 4

1.4 Thesis Outline 5

2 Literature Review

2.1 Hybrid Electric Bus _ 7

2.2 Hybrid Electric Bus Powertrain __ 8

2.2.1 Charge Sustaining Hybrid Electric Powertrain __ 8

2.2.2 Charge Depleting Hybrid Electric Powertrain __ 12

2.2.3 The Integrated Starter Generator Hybrid Electric Powertrain __ 13

2.3 Hybrid Vehicle Control Strategies___ 14

2.3.1 Common Rule-Based Control Strategy __ 14

2.3.2 Optimization Based Control Strategies__ 17

2.3.3 Optimal Predictive Control Strategies _ 20

2.4 Summary _ 23 
3.1 Modeling of the Hybrid Electric Powertrain __ 25

3.1.1 Engine Model __ 25

3.1.2 Electric Machine Model __ 27

3.1.3 Battery Model __ 29

3.1.4 Transmission Model and Torque Coupler Model___ 30

3.1.5 Chassis Model___ 31

3.1.6 The Rule-Based Control Strategy ___ 31

3.2 Validation of Hybrid Bus Model ___ 32

3.3 Summary __ 35

4 DP and ECMS _ 36

4.1 Dynamic Programming ___ 36

4.1.1 The Application of DP in Developing Hybrid Vehicle Control Strategies __ 37

4.1.2 DP Simulation Result ___ 41

4.1.3 The Potential of DP in Improving Cost Function ___ 45

4.1.4 The Trade-off between FE and $\mathrm{NO}_{\mathrm{x}}$ Emissions ___ 46

4.2 Equivalent Consumption Minimization Strategy____ 49

4.2.1 Pontryagin's Minimum Principle ___ 49

4.2.2 Application of ECMS in Developing Hybrid Vehicle Control Strategies __ 50

4.2.3 ECMS Simulation Result __ 53

4.2.4 ECMS with Penalty Function __ 56

4.3 Summary _ 61

5 ECMSwDP 62 
5.1 The ECMSwDP Algorithm __ 63

5.2 Simulation Results and Discussions___ 67

5.2.1 The Extraction of Time-Variant $\lambda(t)$ using the ECMSwDP Model ___ 67

5.2.2 The Study of ECMS with the Extracted $\lambda(t) \_69$

5.2.3 The Study of ECMS Based on the Average of the $\lambda$ Extracted Using ECMSwDP 71

5.2.4 Effect of Road Grade on the ECMSwDP 74

5.2.5 The Effect of $\lambda$ Range on the ECMSwDP 78

5.3 Summary — 81

6 Driving Pattern Identification Model __ 83

6.1 Driving Pattern Features___ 83

6.1.1 The Key Driving Pattern Features for the Description of the Driving Pattern _ 84

6.2 Representative Driving Pattern Development __ 93

6.2.1 The Development of RDP from the 54 Heavy-duty Driving Cycles ___ 93

6.3 The Short Term Prediction for Driving Pattern Features___ 95

6.3.1 The Short Term Prediction Based on Distance Segment ___ 96

6.3.2 The Wavelet Neural Network__ 98

6.3.3 Case Study of the Future Driving Feature Prediction__ 100

6.3.4 Driving Pattern Identification Model __ 101

6.4 Summary _ 103

7 Adaptive ECMS Based on ECMSwDP with Driving Pattern Identification Model_ 104

7.1 Structure of the Proposed Controller _ 104

7.2 Adaptive ECMS _ 105

7.2.1 The A-ECMS Based on $\lambda$ Maps from the Vehicle Operation Data __ 107 
7.2.2 The Study of A-ECMS Based on Different $\lambda$ Map Functions 109

7.3 The A-ECMS Based on $\lambda$ Maps with Driving Pattern Identification 113

7.3.1 The $\lambda(v, a)$ Maps Corresponding to the RDPs 114

7.3.2 The Study of A-ECMS Based on $\lambda$ Maps with Driving Pattern Identification _ 115

7.3.3 The A-ECMS with or without a Penalty Based on the Average of the $\lambda$ Extracted with Driving Pattern Identification 117

7.3.4 The Evaluation of the Control Approaches Examined in This Research 120

7.4 Simulation with a Real-World Route 122

7.5 Summary 125

8 Conclusion and Future Work 127

8.1 Conclusion 127

8.2 Future Work 129

9 Bibliography__ 131

10 Appendix 141

Appendix A: Simulation Results between Rule-Based and DP over the WVU-CSI Cycle 141 Appendix B: The Comparison between Optimal ECMS and DP Simulation Results over the WVU-CSI Cycle 144

Appendix C: The Extracted $\lambda$ Values Obtained by ECMSwDP and the Comparison of ECMS with or without a Penalty $\left(\lambda=\lambda_{0}=\lambda_{\mathrm{ave}}=2.115\right)$ and DP Simulation Results over the WVU-CSI Cycle 146 Appendix D: The Obtained $\lambda$ Maps and the Simulation Results of A-ECMS Based on $\lambda$ maps as a Function of SOC, v, $\mathrm{P}_{\text {req }}$ and an Obtained by ECMSwDP over the WVU-CSI Cycle 148 Appendix E: Simulation Results of A-ECMS Based on $\lambda$ Maps with Driving Pattern Identification over the WVU-CSI Cycle 151 
Appendix F: Simulation Results of A-ECMS with or without a Penalty based on the Average of the $\lambda$ Extracted with the Driving Pattern Identification over the WVU-CSI Cycle 152 


\section{List of Figures}

Figure 2.1 Configuration of series hybrid vehicles

Figure 2.2 Configuration of parallel hybrid vehicles 10

Figure 2.3 Configuration of complex (power split) hybrid vehicles 11

Figure 2.4 Configuration of ISG hybrid vehicles 13

Figure 2.5 Thermostat control strategy for a series HEV (Source: Autonomie vRev12) 15

Figure 2.6 SOC trajectories with the variation of $\lambda$ (Beijing cycle) 20

Figure 2.7 GPS and data logger unit 22

Figure 2.8 Classification and comparison of control strategies 24

Figure 3.1 Topology of the full parallel hybrid electric powertrain 25

Figure 3.2 BSFC map (data from Chen 2010) 27

Figure 3.3 $\mathrm{NO}_{\mathrm{x}}$ emissions map (data from Chen 2010) 27

Figure 3.4 Efficiency map of the EM (data from Chen 2010) 28

Figure 3.5 Internal resistance of the NiMH battery as a function of SOC at $40{ }^{\circ} \mathrm{C}$ (Chen 2010)

Figure 3.6 Internal OCV of the NiMH battery as a function of SOC at $40{ }^{\circ} \mathrm{C}$ (Chen 2010) 29

Figure 3.7 Measured versus simulated vehicle speed and gear number over the Beijing cycle_ 33

Figure 3.8 Simulation of SOC over the Beijing cycle (Initial SOC=51\%) 33

Figure 3.9 Distribution of power source operating points over the Beijing cycle 34

Figure 4.1 Calculation process of DP applied to HEV 40

Figure 4.2 Comparison of trajectories between rule-based and DP over the Beijing cycle 42

Figure 4.3 Distribution of the (a) demand power, (b) rule-based engine power, (c) DP engine power, (d) rule-based battery power and (e) DP battery power over the Beijing cycle. 43 
Figure 4.4 Rule-based and DP power source operation points distribution maps over the Beijing cycle

Figure 4.5 The simulated results of the rule-based controller and DP with an improved cost function over the Beijing cycle 46

Figure 4.6 The trade-off between $\mathrm{FE}$ and $\mathrm{NO}_{\mathrm{x}}$ emissions simulated using DP 47

Figure 4.7 The simulated $\mathrm{NO}_{\mathrm{x}}$ emissions with or without an improved cost function over the Beijing cycle simulated using DP

Figure 4.8 The comparison between DP $(\alpha=\beta=\gamma=0)$ and ECMS with optimal $\lambda$ simulation results over the Beijing cycle

Figure 4.9 DP $(\alpha=\beta=\gamma=0)$ and ECMS with optimal $\lambda$ power source operation points distribution in maps (Beijing cycle) 56

Figure 4.10 Penalty weight used in ECMS to correct the SOC deviation 57

Figure 4.11 The effect of the exponential coefficient on $\mathrm{FE}_{\mathrm{corr}}$ and $\Delta \mathrm{SOC}$ 58

Figure 4.12 Comparison of SOC trajectories obtained by DP and ECMS w/ or w/o penalty in the Beijing cycle $(n=1)$

Figure 4.13 Comparison of $\lambda$ trajectories obtained by ECMS w/ or w/o penalty in the Beijing cycle $(n=1)$ 60

Figure 5.1 The benefit from the combination of DP and ECMS 62

Figure 5.2 The $\lambda$ corresponding databases of the ECMS cost (Beijing cycle) 65

Figure 5.3 Flow chart of ECMSwDP procedures 66

Figure 5.4 The extracted time-variant $\lambda(t)$ obtained by ECMSwDP with or without the improved cost function over the Beijing cycle 68

Figure 5.5 The comparison between the ECMS with the extracted $\lambda(\mathrm{t})$ and DP without the improved cost function over the Beijing cycle

Figure 5.6 ECMS with the extracted $\lambda(\mathrm{t})$ and DP power source operation points distribution in maps (Beijing cycle) 
Figure 5.7 The variation in SOC, $\lambda, \mathrm{P}_{\mathrm{e}}, \mathrm{P}_{\mathrm{b}}$ over the Beijing cycle simulated with ECMS w/ and w/o penalty function, and DP

Figure 5.8 Beijing cycle sin wave function and elevation $G_{m}=3 \% D_{p} / D_{t}=0$ 75

Figure 5.9 Synthetic constant speed with sine wave maximum grade from $1 \%$ to $10 \%$ 76

Figure 5.10 Comparison of SOC trajectories obtained by ECMSwDP over the synthetic driving cycle 76

Figure 5.11 The relationship between the minimum SOC and the grade by ECMSwDP over synthetic driving cycle 77

Figure 5.12 Comparison of $\lambda$ trajectories obtained by ECMSwDP over a sine wave with a maximum grade from $1 \%$ to $10 \%$ and a cruising speed of $14 \mathrm{mpg}$ 78

Figure 5.13 Effect of $\lambda$ range on the $\mathrm{FE}$ and $\mathrm{NO}_{\mathrm{x}}$ Emissions 79

Figure 5.14 Comparison of the different $\lambda$ ranges obtained by ECMSwDP over the Beijing cycle 80

Figure 6.1 The eigenvalues associated with a component 86

Figure 6.2 Flow chart of extracting key driving pattern features 90

Figure 6.3 Validation of the models under different variables 92

Figure 6.4 Three-dimensional contour plot of the HERPI with variables average acceleration and speed 93

Figure 6.5 Six representative standard driving cycles 95

Figure 6.6 The distance based segment 96

Figure 6.7 Illustration of the distance based segment prediction diagram 97

Figure 6.8 The architecture of the WNN model 99

Figure 6.9 The average speed predicted using different distance segment 101

Figure 6.10 The architecture of the driving pattern identification model 102

Figure 6.11 The identified driving patterns of one session from Rt6 102 
Figure 7.1 Flow chart of real-time optimal control strategy of hybrid transit bus based on predicted driving patterns 105

Figure 7.2 The schematic diagram of SOC trajectories derived using ECMS and A-ECMS _ 106

Figure 7.3 A-ECMS based on SOC feedback___ 107

Figure 7.4 A-ECMS based on $\lambda$ map ___ 107

Figure 7.5 The $\lambda$ maps as a function of SOC, $v, P_{r e q}$ and $a$ derived using ECMSwDP over the Beijing cycle 109

Figure 7.6 The comparison between A-ECMS based on $\lambda$ maps as a function of $(S O C, v)$, $\left(S O C, P_{r e q}\right)$ and $(S O C, a)$ obtained and DP simulation results over the Beijing cycle _ 110

Figure 7.7 The comparison between A-ECMS based on $\lambda$ maps as a function of $\left(v, P_{\text {req }}\right),\left(P_{\text {req, }}\right.$ a) and $(v, a)$ obtained and DP simulation results over the Beijing cycle 111

Figure 7.8 A-ECMS based on $\lambda$ maps with driving pattern identification 113

Figure $7.9 \lambda$ maps of the six RDPs

Figure 7.10 The comparison between A-ECMS based on $\lambda$ maps with the driving pattern identification and ECMSwDP simulation results over the Beijing cycle 116

Figure 7.11 A-ECMS based on the average of the $\lambda$ values extracted with driving pattern identification

Figure 7.12 The comparison between A-ECMS with or without a penalty based on the average of the $\lambda$ extracted with the driving pattern identification and ECMSwDP simulation results over the Beijing cycle

Figure 7.13 Transit bus Route 6 in the SEAT district of Preston, CT

Figure 7.14 The actual Rt6 cycle sampled in SEAT

Figure 7.15 The comparison of simulation results from the proposed A-ECMS based on $\lambda$ maps of the predicted RDPs, the A-ECMS with a penalty based on the average of the $\lambda$ extracted with driving pattern identification, rule-based, and DP over Rt6 in SEAT 124

Figure A. 1 WVU-CSI cycle 
Figure A. 2 The operation parameters obtained with rule-based and DP $(\alpha=\beta=\gamma=0)$ in the WVU-CSI cycle

Figure A. 3 Rule-based and DP $(\alpha=\beta=\gamma=0)$ power source operation points distribution in maps (WVU-CSI cycle)

Figure A. 4 Distribution of the (a) demand power, (b) rule-based engine power, (c) DP engine power, (d) rule-based battery power and (e) DP battery power during the WVU-CSI cycle

Figure B. 1 The operation parameters obtained with ECMS $\left(\lambda_{\text {opt }}=2.655\right)$ and DP $(\alpha=\beta=\gamma=0)$ (WVU-CSI cycle)

Figure B. 2 Optimal ECMS $\left(\lambda_{\text {opt }}=2.655\right)$ and DP $(\alpha=\beta=\gamma=0)$ power source operation points distribution in maps (WVU-CSI cycle)

Figure C. 1 The $\lambda$ values extracted using ECMSwDP over the WVU-CSI cycle

Figure C. 2 The operation parameters obtained using ECMS with or without penalty function $\left(\lambda_{\mathrm{ave}}=2.115\right)$ and ECMSwDP $(\alpha=\beta=\gamma=0)$ simulation results (WVU-CSI cycle)

Figure D. 1 The $\lambda$ maps as a function of $S O C, v, P_{r e q}$ and $a$ obtained by ECMSwDP over the WVU-CSI cycle

Figure D. 2 The operation parameters obtained with The operation parameters obtained with The operation parameters obtained with A-ECMS based on $\lambda$ maps as a function of $S O C, v, P_{r e q}$ and $a$ obtained by ECMSwDP and DP simulation results over the WVUCSI cycle

Figure D. 3 The comparison between A-ECMS based on $\lambda$ maps as function of $v, P_{r e q}$ and $a$ obtained by ECMSwDP and DP simulation results over the WVU-CSI cycle

Figure E. 1 The comparison between A-ECMS based on $\lambda$ maps with driving pattern identification and ECMSwDP simulation results over the WVU-CSI cycle 
Figure F. 1 The comparison between A-ECMS with or without a penalty based on the average of the $\lambda$ extracted with the driving pattern identification and ECMSwDP simulation results over the WVU-CSI cycle 


\section{List of Tables}

Table 3.1 Basic specifications of the parallel hybrid transit bus (Zeng 2005) __ 26

Table 3.2 Validation results of the hybrid bus model (Zeng 2005, Chen 2010)___ 35

Table 4.1 Variables and grids of DP algorithm ___ 41

Table 4.2 DP and rule-based simulation results for Beijing cycle ___ 45

Table 4.3 DP with or without penalty and rule-based simulation results for the Beijing cycle _ 49

Table 4.4 DP $(\alpha=\beta=\gamma=0)$ and ECMS with optimal $\lambda$ simulation results over the Beijing cycle 54

Table 4.5 Comparison of corrected FE obtained by DP and ECMS w/ or w/o penalty in the Beijing cycle

Table 5.1 Variables and grids of ECMSwDP algorithm___ 64

Table 5.2 Comparison of the ECMS with the extracted $\lambda(t)$ and DP $(\alpha=\beta=\gamma=0)$ simulation results in the Beijing cycle

Table 5.3 The simulation results of the ECMS with or without penalty function and DP in the Beijing cycle

Table 5.4 The simulation results of the ECMS with or without penalty function and DP in the WVU-CSI cycle _ 74

Table 5.5 Simulation results of different $\lambda$ range obtained by ECMSwDP over the Beijing cycle 80

Table 6.1 The driving pattern features 84

Table 6.2 The driving features of the current 54 heavy-duty drive cycles collected 87

Table 6.3 Component matrix of PCA 89

Table 6.4 Stepwise regression coefficients 91

Table 6.5 Cluster membership and the distance 94

Table 6.6 Identification features of six RDPs 94 
Table 7.1 The fuel economy and changes in SOC observed using A-ECMS with $\lambda$ maps as a function of two of the four parameters including $S O C, v, P_{r e q}$ and $a$ over the Beijing cycle

Table 7.2 The fuel economy and changes in SOC observed using A-ECMS with $\lambda$ maps as a function of two of the four parameters including $S O C, v, P_{r e q}$ and $a$ over the WVUCSI cycle

Table 7.3 The simulation results of A-ECMS based on its own $\lambda$ maps corresponding to the six RDPs

Table 7.4 Simulation results of A-ECMS based on $\lambda$ maps with the driving pattern identification over the Beijing cycle

Table 7.5 Simulation results of A-ECMS based on $\lambda$ maps with the driving pattern identification over the WVU-CSI cycle

Table 7.6 The average of the $\lambda$ values extracted corresponding to the six RDPs

Table 7.7 Simulation results by A-ECMS with or without a penalty based on the average of the $\lambda$ extracted with the driving pattern identification over the Beijing cycle

Table 7.8 Simulation results of A-ECMS with or without a penalty based on the average of the $\lambda$ extracted with the driving pattern identification over the WVU-CSI cycle

Table 7.9 Comparison of fuel economy and the change in SOC simulated using different control approaches over the Beijing cycle

Table 7.10 Comparison of fuel economy and the change in SOC simulated using different control approaches over the revised WVU-CSI cycle

Table 7.11 Comparison of fuel economy and the change in SOC for different control approaches over the actual Rt6 operational data

Table A. 1 DP $(\alpha=\beta=\gamma=0)$ and rule-based simulation results for the WVU-CSI cycle

Table B. 1 DP $(\alpha=\beta=\gamma=0)$ and Optimal ECMS $(\lambda \circ p t=2.655)$ simulation results for the WVUCSI cycle 


\section{Nomenclature}

\section{$\underline{\text { Abbreviations }}$}

\begin{tabular}{|c|c|}
\hline A-ECMS & Adaptive Equivalent Consumption Minimization Strategy \\
\hline AER & All Electric Range \\
\hline ARIMA & Auto Regressive Integrated Moving Average \\
\hline ARMA & Autoregressive Moving Average \\
\hline ANL & Argonne National Laboratory \\
\hline Beijing & Beijing Cycle \\
\hline CAN bus & Vehicle Controller Area Network \\
\hline $\mathrm{CD}$ & Charge Depleting \\
\hline $\mathrm{CS}$ & Charge Sustaining \\
\hline CUB & China's Urban Bus cycle \\
\hline DP & Dynamic Programming \\
\hline DPI & Driving Pattern Identification \\
\hline ECMS & Equivalent Consumption Minimization Strategy \\
\hline ECMSwDP & ECMS with DP Combined Model \\
\hline $\mathrm{ECU}$ & Engine Control Unit \\
\hline EM & Electric Machine \\
\hline ESS & Energy Storage System \\
\hline $\mathrm{EV}$ & Electric Vehicle \\
\hline $\mathrm{FC}$ & Fuel Consumption \\
\hline $\mathrm{FE}$ & Fuel Economy \\
\hline GA & Genetic Algorithm \\
\hline GHG & Greenhouse Gas \\
\hline GIS & Geographic Information System \\
\hline GPS & Global Positioning System \\
\hline HEBs & Hybrid Electric Buses \\
\hline $\mathrm{HEV}$ & Hybrid Electric Vehicle \\
\hline HERPI & Hybrid Energy Recovery Potential Index \\
\hline
\end{tabular}




$\begin{array}{ll}\text { ICE } & \text { Internal Combustion Engine } \\ \text { ISG } & \text { Integrated Starter Generator } \\ \text { ITS } & \text { Intelligent Transportation Systems } \\ \text { MPG } & \text { Mile per Gallon } \\ \text { NN } & \text { Neutral Network } \\ \text { NaN } & \text { Not-a-Number } \\ \text { NO }_{x} & \text { Oxides of Nitrogen } \\ \text { PHEVs } & \text { Plug-in Hybrid Electric Vehicles } \\ \text { PKE } & \text { Positive Acceleration Kinetic Energy } \\ \text { PMP } & \text { Pontryagin's Minimum Principle } \\ \text { PTBI } & \text { Potential to the Best Improvement } \\ \text { RDP } & \text { Representative Driving Pattern } \\ \text { SEAT } & \text { Southeast Area Transit } \\ \text { SOC } & \text { State of Charge } \\ \text { V2I } & \text { Vehicle to Infrastructure } \\ \text { V2V } & \text { Vehicle to Vehicle } \\ \text { WNN } & \text { Wavelet Neural Network } \\ \text { WVU } & \text { West Virginia University } \\ \text { WVU-CSI } & \text { Combined the WVU City cycle, WVU Suburban cycle and WVU } \\ & \text { Interstate cycle }\end{array}$

\section{$\underline{\text { Symbols }}$}

$\begin{array}{ll}C_{k} & \text { Predicted driving pattern, Equation } 6.9 \\ D_{t} & \text { Total distance of drive cycle, Equation } 5.1 \\ D_{P} & \text { The phase shift of the wave, Equation } 5.1 \\ E_{B R} & \text { The potential braking energy, Equation } 0.1 \\ E_{t r a c} & \text { The total tractive energy, Equation } 0.2 \\ G_{m} & \text { Maximum/minimum road grade, Equation } 5.1 \\ H_{f} & \text { The lower heating value of the fuel, Equation } 4.5 \\ I_{b} & \text { The current of NiMH battery }\end{array}$




\begin{tabular}{|c|c|}
\hline$J$ & The cost-to-go function of DP, Equation 4.2 \\
\hline$k$ & The discrete time index of DP \\
\hline$L$ & The state grid of DP \\
\hline M & The control grid of DP \\
\hline$\dot{m}_{f u e l / N O_{x}}$ & Fuel and $\mathrm{NO}_{\mathrm{x}}$ rate, Equation 3.1 \\
\hline$N$ & The time grid of DP \\
\hline$P$ & Equivalent factor grid in ECMSwDP \\
\hline$P_{a c c}$ & Inertial Load in road load equation \\
\hline$P_{g d}$ & Elevation Load in road load equation \\
\hline$P_{b}$ & Battery power \\
\hline$P_{e}$ & Engine power \\
\hline$P_{e m}$ & Required electrical power \\
\hline$P_{r}$ & Rolling resistance \\
\hline$P_{w d}$ & Aerodynamic drag in road load equation \\
\hline$P T B I_{F E_{c o r r}}$ & Potential to the best improvement \\
\hline$Q_{b}$ & Nominal battery capacity \\
\hline$R_{i}$ & Internal resistance of NiMH battery \\
\hline$T_{e m}$ & Electric machine torque \\
\hline$T_{\text {em,req }}$ & Requested electric machine torque \\
\hline$T_{i c e}$ & Engine torque \\
\hline$T_{\text {req }}$ & Vehicle request torque \\
\hline$u(k), x(k)$ & The control and state vector of DP, Equation 4.1, Equation 4.2 \\
\hline$V_{o c}$ & Open-circuit voltage of NiMH battery \\
\hline$v_{\text {req }}$ & Vehicle request speed \\
\hline$\alpha, \beta, \gamma$ & $\begin{array}{l}\text { The weighting factor of battery current, difference engine on or off status } \\
\text { and } \mathrm{NO}_{\mathrm{x}} \text { emissions in DP cost function, Equation } 4.11\end{array}$ \\
\hline$\gamma_{c}, \gamma_{f}, \gamma_{t}$ & Gear ratio of coupling, final drive and transmission, Equation 3.8 \\
\hline$\eta_{e m}$ & Electric machine efficiency \\
\hline$\eta_{\text {path }}$ & $\begin{array}{l}\text { The efficiency of the drivetrain used in regenerating/discharging the } \\
\text { battery }\end{array}$ \\
\hline
\end{tabular}




$\begin{array}{ll}\omega_{\text {em }} & \text { Electric machine speed } \\ \omega_{\text {ice }} & \text { Engine speed } \\ \lambda & \text { Equivalent factor of ECMS } \\ \lambda_{\text {ave }, w / i} & \text { The extracted averages of } \lambda \text { with improved cost function } \\ \lambda_{\text {ave }, w / o i} & \text { The extracted averages of } \lambda \text { without improved cost function } \\ \lambda_{\text {opt }} & \text { Optimal equivalent factor } \\ \lambda_{0} & \text { Initial equivalent factor in ECMS with penalty } \\ \sigma & \text { Mass coefficient considering inertia effect. } \\ \Delta E_{\text {on } / o f f} & \text { The difference engine on or off status in DP cost function, Equation } 4.11 \\ \Delta S O C & \text { The change in SOC of battery } \\ \tau & \text { Unit convert parameter, Equation } 4.31\end{array}$

\section{$\underline{\text { Subscripts }}$}

$\begin{array}{ll}\text { ave } & \text { Average value } \\ \text { corr } & \text { Corrected } \\ \text { em } & \text { Electric machine } \\ \text { eqv } & \text { Equivalent } \\ \min & \text { Minimum value } \\ \max & \text { Maximum value } \\ r e f & \text { Reference value, equation } \\ w / & \text { With } \\ w / o & \text { Without }\end{array}$




\section{Introduction}

\subsection{Background}

The ever increasing fuel consumption (FC) and emissions of pollutants, as well as continuous concern for greenhouse gas (GHG) emissions have led to the development of more efficient and cleaner transportation technologies. Past researches have focused on technologies capable of significantly improving the engine thermal efficiency, application of after-treatment systems, and further reduction of vehicle energy consumption through better design of vehicle dynamics. A more holistic approach in reducing the FC and GHG emissions of on-road vehicles is the development and application of hybrid vehicle technologies. The application of hybrid technology decreases FC by recovering and reusing energy that, for conventional vehicles, is lost during the vehicle braking process (Sundstrom and Guzzella 2009). FC is reduced further through hybrid technology by shifting the engine operation from a low to high efficiency zone, storing electrical energy in a relatively large battery (which acts as an energy buffer), downsizing the engine, the electrification of the auxiliary system, and the automatic start/shutoff of the engine during vehicle stop and go mode (Panday and Bansal 2014).

Hybrid vehicles can be categorized as mild hybrid vehicles, or full hybrid vehicles. A mild hybrid vehicle is essentially a conventional vehicle that has been modified to allow for electrification of the auxiliary system, which makes it possible to shut down the internal combustion engine (ICE) during coasting and idle periods, and to restart it when propulsion power is requested such as when acceleration is needed. In comparison, a full hybrid vehicle, sometimes called a strong hybrid, is a vehicle that can run on either the ICE, the electric motor only, or a combination of both. A full hybrid bus usually can recovery the vehicle's kinetic energy during the braking process, convert it to electrical energy, and 
store it in the battery for vehicle propulsion or powering the auxiliary system. The electric motor of a hybrid vehicle can either power the vehicle by itself or work together with the ICE to provide the energy needed for vehicle operation. Traditionally, a hybrid electric vehicle (HEV) can be classified as one of three types: series, parallel, and combination.

i. Series hybrid vehicle

The series hybrid vehicle is driven by an electric motor, which is powered by the battery and electricity produced by the onboard ICE-alternator system. In a series hybrid vehicle, the ICE is not mechanically connected to the propulsion wheels but run as an onboard generator.

ii. Parallel hybrid vehicle

In a parallel hybrid vehicle, both the ICE and electric motor are mechanically connected in parallel with the wheels. A parallel hybrid vehicle can operate with the electric motor only, ICE only, or both. A parallel hybrid vehicle generally falls into two types classified by the layout of motor: pre-transmission and post-transmission.

iii. Combination hybrid vehicle

Combination hybrid vehicle incorporates the features of both a series and parallel HEV. An additional mechanical connection exists between the ICE and transmission compared with the series hybrid, and an additional generator is installed between the ICE and power converter compared with the parallel hybrid.

A hybrid vehicle usually achieves its best performance when operated with frequent acceleration, decelerations, stop and goes, and long idle periods, which are characteristics of a vehicle that operates run in heavily populated urban areas. The high cost of hybrid vehicles makes them more suitable for vehicles which run for a long time each day. The operation of a vehicle with a fixed route through urban areas makes the hybrid vehicle achieve its best potential in reducing FC and GHG because of the frequent start-stops, 
accelerations and deceleration operations. Among the vehicles considered, transit buses are recognized as the one most suitable for hybridization. Hybrid electric buses (HEBs) are defined as transit buses carrying at least two sources of motive energy on board and using electrical energy to provide partial or complete drive power to the vehicle's wheels (Salmasi 2007). There is an infinite number of HEB driveline configurations based on a combination of 4 critical components: fuel converters (including ICEs, generators, and electric motors), drive-trains, auxiliaries, and elements for the storage of energy regenerated during the braking process. The physical configuration is only one of the important factors which influences the fuel economy and emissions. The energy management strategy also can have significant impact on FC and emissions. The availability of two different energy storage systems, two power systems, an electrified auxiliary system, different operation modes, and management of the battery charging or discharging, makes the control of the hybrid system much more complex than that of traditional vehicles. This requires the development and application of more advanced HEV control strategies than those of traditional vehicles.

\subsection{Motivation}

The control strategy of a HEV has been an active research area in the past decades. The main goal is to maximize the vehicle fuel economy (FE) rated in miles per gallon (mpg) and minimize emissions while also satisfying the expected driving performance. The control strategies can be categorized as one of the following three types: (1) The heuristic rule-based controllers, which are widely implemented in current HEVs but cannot guarantee the optimal result (Pisu and Rizzoni 2007); (2) The global optimality method of the dynamic programming (DP) (Sundstrom and Guzzella 2009) controller can obtain the global optimal solution, but it requires the pre-known driving cycle information and there is a heavy calculation cost. Accordingly, the DP controller traditionally cannot be used in 
real-time control, which is often implemented as a benchmark for the development of the energy management controller; (3) The equivalent consumption minimization strategy (ECMS) (Paganelli, et al. 2002), which is a compromise between the optimal and real-time control approaches. The ECMS is considered to be a local optimization control approach and is widely used in HEV energy management, but ECMS only uses a randomly selected constant equivalent factor $(\lambda)$, which can't guarantee charge sustainability of the entire driving cycle. In order to apply in real-time controllers, some researchers proposed the adaptive equivalent consumption minimization strategy (A-ECMS) (Onori, Serrao and Rizzoni 2010), which changes $\lambda$ based on the state of charge (SOC) of battery and maintains the SOC around the reference variation. However, A-ECMS cannot guarantee the near-optimal and desired SOC of battery under active driving conditions such as the up/down hill driving phase (Han, Park and Kum 2014). The optimal control strategy is performed over a fixed driving cycle, by assuming the future driving conditions are known. However, the driving pattern of a transit bus operated on a fixed route is influenced by the traffic volume, road surface, local activities, unexpected environments, extreme weather, and the operation of the accessory system which largely depends on the ambient conditions. As reported by Ericsson, prior knowledge of the driving pattern and environment can make a significant impact on the potential of the hybrid vehicle in reducing fuel consumption and exhaust emissions if the control strategies can be adjusted to address the future driving patterns.

\subsection{Objectives of Thesis}

The objective of this study is to develop the real-time optimal control strategies of a parallel HEB. The following are the main tasks to be completed:

- To develop a HEB model using Autonomie as a platform and validate it against experimental data; 
- To develop the code for DP and ECMS and examine the potential of DP and ECMS in improving the performance of a simulated parallel HEB;

- To develop a model combining ECMS with DP for the extraction of the optimal $\lambda$, which might be a function of battery SOC, vehicle operation features, route information, etc.;

- To develop a model capable of predicting the operation features of HEBs operated on a fixed route, as well as a model capable of identifying the representative driving patterns with the known or predicted driving features;

- To develop an A-ECMS based on the $\lambda$ extracted with the current vehicle operation data and the predicted future operation features;

- To numerically compare the impact of the control strategies developed using rulebased control, ECMS, ECMSwDP, and A-ECMS on the fuel economy of a simulated parallel HEB.

\subsection{Thesis Outline}

There are 8 chapters in this thesis. The current chapter briefly introduces the outline of hybrid vehicles, the motivation, and the main objectives of this research.

Chapter 2 presents the literature review relating to HEVs and control technologies, and identifies the need for developing real-time optimal control strategies for hybrid vehicles. Chapter 3 introduces the development of a parallel HEB model and its validation against experimental data reported in the literature. This model is used as the platform for the development and examination of the control strategies to be discussed and developed in this research. 
Chapter 4 presents the DP and ECMS algorithms and examines the impact of the control strategies developed using DP and ECMS in improving the fuel economy of a parallel HEB operated over the Beijing cycle and WVU-CSI cycle.

Chapter 5 develops an algorithm combining ECMS with DP, noted as ECMSwDP. The main outcome of ECMSwDP is the optimal $\lambda(t)$ or $\lambda$ map for real time adaptive-ECMS.

Chapter 6 reports the development of a driving feature prediction model, and a driving pattern identification model.

Chapter 7 proposes the development of an A-ECMS model capable of generating the robust, real-time and suboptimal control strategies for HEBs. The impact of control strategies developed using ECMS, ECMSwDP and A-ECMS on the fuel economy will be examined and discussed.

Chapter 8 concludes this dissertation with a brief summary of work completed, major contributions made, and conclusions drawn based on the results obtained in this research. The future research work that should be conducted is recommended. 


\section{Literature Review}

\subsection{Hybrid Electric Bus}

Improvement of the public transportation system has been recognized as one of the possible solutions to the ever-increasing traffic congestion and air pollution in metropolitan areas such as Washington D.C., USA and Beijing, China. The diesel-fueled transit buses with conventional drivetrain are known to be high in fuel cost and emissions. Transit buses in the USA consumed approximately 2,233 million gallons of diesel fuel in 2014 (U.S. Department of Energy 2014). Further reducing the FC and exhaust emissions from transit buses has been the focus of transit agencies.

Transit buses have been identified as one platform that is suitable for hybridization (Williamson, Wirasingha and Emadi 2006).

- The large space of HEBs make it possible to integrate the battery size and electric powertrain system.

- With high frequency start-stops and deceleration driving conditions, HEBs can recover more energy through a regenerative braking system than vehicles operated at a relatively constant speed.

- Operated at fixed route and fixed schedule, which makes its operation features more recognizable and predictable than other vehicles. This makes it possible to develop specific control strategies unique to a bus route or transit bus agency.

- A transit bus usually operates 8 hours or more each day, and 5 or more days a week which makes it possible for transit agencies to reduce $\mathrm{FC}$, as well as $\mathrm{GHG}$ emissions at reduced operation cost. Transit buses are usually designed and fabricated to serve for 12 years or more in US. This makes it easier to have a reasonably short payback period. 
- The energy storage system (ESS) can be used as a buffer for extremely transient driver power demanded HEBs, which can make the vehicle operate with a smaller size engine with less transient modes on its more efficient zones. This is desirable but usually difficult to achieve with a conventional bus.

\subsection{Hybrid Electric Bus Powertrain}

The charge sustaining (CS) and charge depleting (CD) configurations are in a full hybrid, the engine and motor offer comparable levels of propulsion power and can work independently or in conjunction with each other. The ISG configuration is a mild hybrid which has an oversized starter motor that makes it possible to turn off the engine when the bus is coasting, braking or stopped, then restarts the motor quickly and seamlessly when vehicle needs power for actions such as acceleration. The application of the ISG configuration makes it possible to shut down engine during vehicle coasting and stop (Eick 2012, C. Chan 2002).

\subsubsection{Charge Sustaining Hybrid Electric Powertrain}

In a CS hybrid powertrain, to avoid costly battery replacements during the expected life of the vehicle, there must be enough mechanical power produced by the engine to drive the bus over a reasonable route and the batteries must endure an extended period of vehicle operation.

\section{Series Hybrid Electric Powertrain}

The wheels of a series hybrid electric powertrain are propelled by a traction motor powered by electrical energy stored in the battery or generated by the ICE. The electrical energy recovered during the braking process is also stored in the battery for future application in powering the traction motor and the electrical auxiliary system. Since the ICE is not 
directly mechanically connected to the wheels, it can operate at an engine load and speed independent of vehicle power request, speed and gear ratio, which makes it possible for the ICE to operate at the better efficiency. The ICE can even be switched off for short periods of time for an engine free operation of the bus. Figure 2.1 shows the series hybrid electric powertrain configuration.

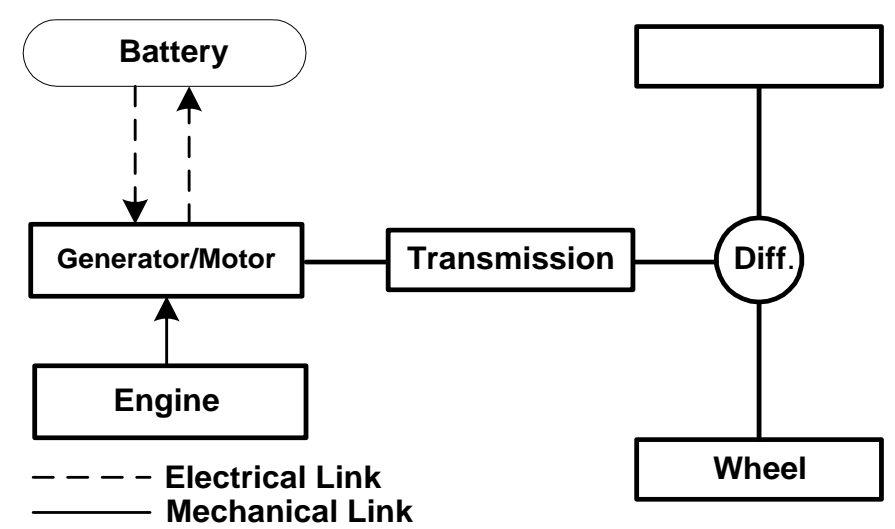

Figure 2.1 Configuration of series hybrid vehicles

However, as the electric machine is the only drive mechanism connected to the wheels, a large traction motor is necessary. Another weakness of the series hybrid configuration is that energy is first converted from mechanical power to electricity by a generator and later on converted to mechanical work by a motor. The combined efficiency of the generatorbattery-motor system is about $80 \%$ (Eick 2012), indicating a loss of $20 \%$ of the mechanical work to waste heat in ESS. The dissipation of thermal energy generated by the electrical system, especially the battery, consumes energy and requests the development of complex thermal management systems. Some examples of companies that manufacture series hybrid buses include BAE Systems, ISE and E-bus which sell 40-foot and 22-foot series hybrid electric transit buses in the U.S. market (County 2005). 


\section{Parallel Hybrid Electric Powertrain}

The parallel hybrid vehicle is referred to in powertrain technology as having the engine and electric motor mechanically connected in parallel to the transmission. Parallel hybrid vehicles can operate on engine only mode, electric only mode, or both.

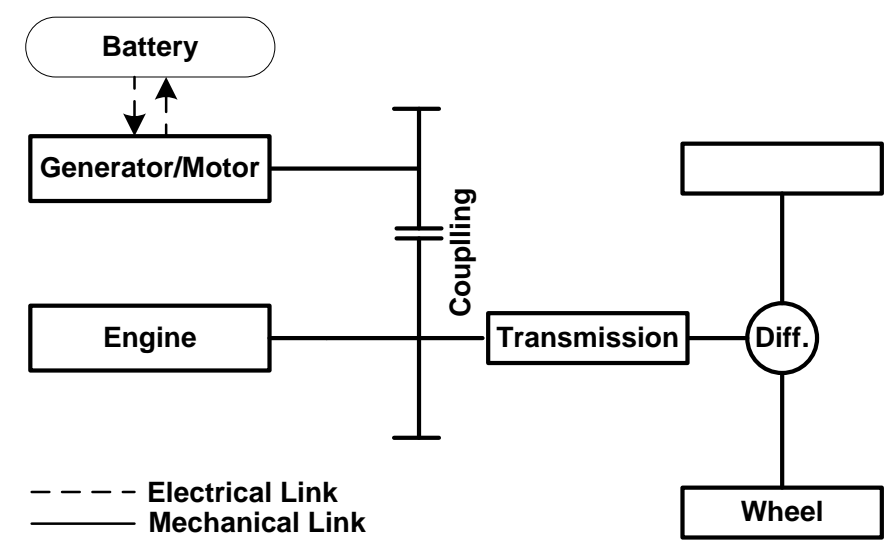

A

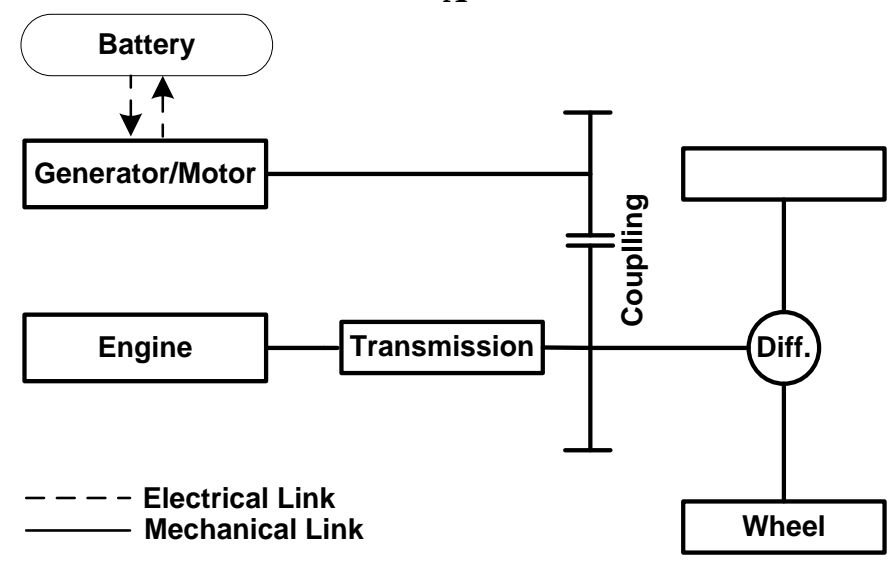

B

Figure 2.2 Configuration of parallel hybrid vehicles

The ICE and electric machine of a parallel hybrid electric powertrain are mechanically connected to the transmission. This makes the parallel system more complex but more efficient than a series hybrid configuration during most operating conditions. This is due to the elimination of the mechanical work to electricity, and electricity to mechanical work conversion loop. 
There are two types of parallel hybrid vehicles, which are classified by the layout of the motor: pre-transmission and post-transmission parallel hybrid vehicle. A pre-transmission motor, shown in Figure 2.2-A, operates over a smaller speed range than a post-transmission motor, and it can effectively provide more torque to the drive wheels at low speed. However, the motor power of a post-transmission configuration (Figure 2.2-B) doesn't need to pass through the transmission, so it can get a higher efficiency for driving the wheels or recapturing braking energy.

Eaton manufactures parallel hybrid systems for medium/heavy-duty trucks and 40 to 60foot transit buses. The chassis configuration used for simulation studies in this thesis is the pre-transmission parallel hybrid electric powertrain.

\section{Complex Hybrid Electric Powertrain}

The complex hybrid architecture is to some extent a combination of the series and parallel hybrid bus. Compared to the series configuration, a mechanical link between the generator and the electric motor is added. A complex hybrid has one more generator than a parallel hybrid. With this design, it is possible to combine the advantages of both the series and parallel HEV configurations, but the design and control are much more intricate.

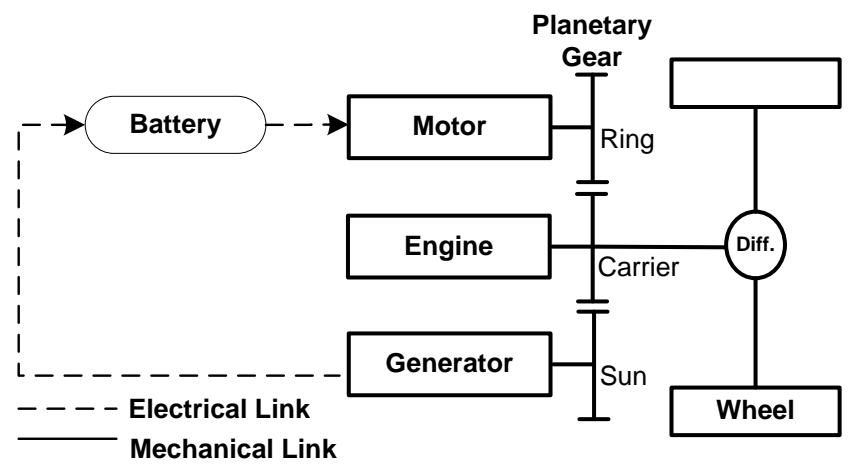

Figure 2.3 Configuration of complex (power split) hybrid vehicles

The Allison buses currently on the market employ a planetary system with a range shift, which has been termed a split parallel (Clark, Zhen and Wayne 2009). In the light-duty 
market, the Toyota Prius has demonstrated the best FE with a planetary system, as shown in Figure 2.3.

\subsubsection{Charge Depleting Hybrid Electric Powertrain}

A charge depleting hybrid system refers to a mode of vehicle operation powered mainly by the electrical energy stored in the battery pack. Battery electric vehicles operate solely in this mode. Most plug-in hybrid electric vehicles (PHEVs) operate in CD mode at startup, consume electricity only, and later on switch to CS mode after the battery has reached its minimum SOC threshold, and exhausted the vehicle's all electric range (AER) (Markel 2006).

The electrical energy stored in the battery pack of a PHEV is supplied from an off-vehicle source when the vehicle is parked, while the electricity recovered during the braking process can also be stored in the battery but only contribute to a very small portion of the total energy stored in battery. PHEV typically utilizes the stored power to drive the vehicle in electric only mode for a certain distance, so the battery is normally larger than that of a CS hybrid powertrain (Zhang, Mi and Zhang 2011).

\section{Series Plug-in Hybrid Electric Powertrain}

A series plug-in hybrid electric powertrain basically consists of an electric motor to propel the vehicle. When the battery charge is depleted, the onboard generator system provides electrical power for charging the battery or powering the motor to propel the vehicle. In a series plug-in powertrain, there is no direct mechanical connection between the engine and drive wheels, so the ICE can work at its high efficient zone when powering the generator. Ebus Inc. has produced series PHEV transit buses that utilize a diesel powered micro turbine to drive the on-board generator and provide additional operation range once the primary charge in the battery pack is depleted (Vyas, Santini and Johnson 2009, Eick 2012). 


\section{Parallel Plug-in Hybrid Electric Powertrain}

In the parallel plug-in hybrid electric powertrain, there is a mechanical linkage from the ICE to the drive wheels that can assist the electric motor for aggressive acceleration or high speeds. Unlike the parallel HEVs, parallel PHEVs normally have a significantly greater AER before the ICE needs to be turned on. Azure Dynamics provides parallel plug-in hybrid powertrains for Ford's E-Series and F-Series truck chassis (Eick 2012).

Parallel and Series powertrain configurations are the two primary options for PHEVs. As these types of PHEV operate by drawing power from the battery, the PHEV is considered to be a CD mode. When operating in CD mode without any assistance from the ICE, the PHEV is operating as an electric vehicle. When the ICE is turned on, the PHEV operates on CS mode, similar to traditional hybrid vehicles.

\subsubsection{The Integrated Starter Generator Hybrid Electric Powertrain}

The ISG hybrid electric powertrain is primarily an electric motor machine mounted on the crankshaft between the ICE and transmission. The ISG architecture is categorized into parallel and mild hybrid configurations because of its relatively small size of motor adopted (Mao, et al. 2008), illustrated in Figure 2.4.

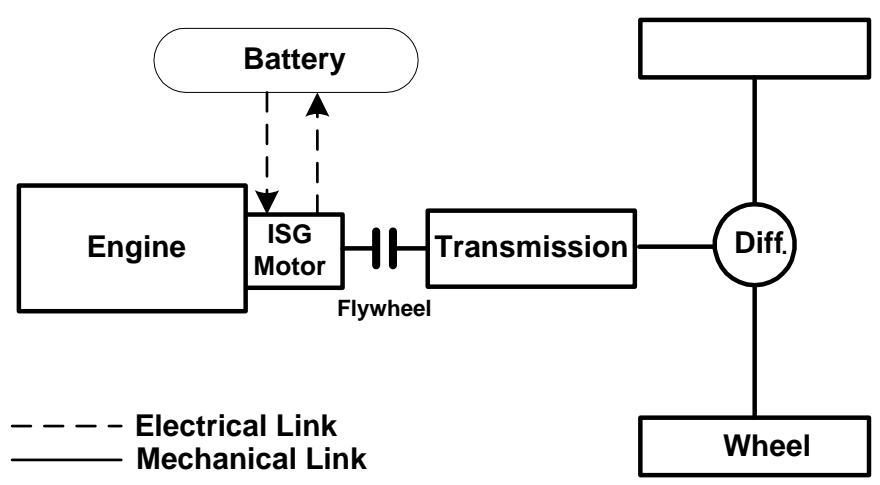

Figure 2.4 Configuration of ISG hybrid vehicles

This mild hybrid, or stop-start system, shuts off the engine when the vehicle is at rest in order to save fuel, and automatically restarts it when the driver requests acceleration. This 
is typically accomplished through an electric motor/generator that can assist the ICE during acceleration and recover electrical energy and send it to the battery pack during deceleration, but does not have enough power to propel the vehicle on its own. Mild hybrids typically achieve FC savings of 8 to 12 percent if designed properly and operate on frequent stop and go operation (Eick 2012).

\subsection{Hybrid Vehicle Control Strategies}

A major challenge for the improvement of hybrid vehicles is the coordination of multiple energy sources to minimize FC and exhaust emissions, to sustain battery energy, and to achieve the drivability and operational ability expected by the driver. The actions taken by the control strategies play a critical role in determining the performance and efficiency of a hybrid vehicle (Onori and Serrao 2011, Sundstrom and Guzzella 2009, Paganelli, et al. 2002, Pisu and Rizzoni 2007). Hybrid powertrain control strategies can be classified in accordance of properties optimization (global/local optimality), computational burden (online/offline control), and the use of prediction model for the forgoing operation features with the prior knowledge of routes (Salmasi 2007). Typically, there are three types of control strategies: common rule-based control strategy, optimization based control strategies, and optimal predictive control strategies.

\subsubsection{Common Rule-Based Control Strategy}

The common rule-based control strategy selects the operation modes according to the predefined rules designed based on heuristics, intuition and human expertise (engineering knowledge), without prior knowledge of the route (Wirasingha and Emadi 2011, Tie and Tan 2013, Panday and Bansal 2014). The rule-based control strategies for hybrid vehicles can be classified into the deterministic rule-based method and fuzzy rule-based method. 


\section{The Deterministic Rule-Based Methods}

Deterministic rule-based controllers select certain operation modes such as electric vehicle (EV) mode, engine mode, engine charge mode, power split mode and performance limited mode based on a set of rules and instantaneous inputs of driving condition that have been defined and implemented prior to actual operation (Tie and Tan 2013). Deterministic rulebased methods consist of thermostat (on/off) strategy, power follower (baseline) control strategy, modified power follower strategy and state machine based strategy (Bayindir, Gözüküçük and Teke 2011, Wirasingha and Emadi 2011).

The simplest deterministic rule-based controller would be the thermostat controller as shown in Figure 2.5. It operates on the simple principle that the ICE will be turned on or off based on the SOC and the torque request of the vehicle. The controller has been successful used in a series hybrid drive train, where the electric motor provides all the propulsion, and the ICE is required to sustain a defined SOC. The thermostat control strategy is robust, simple and easy to control but leads to high frequency on and off (charging and discharging) cycles of the engine and battery.

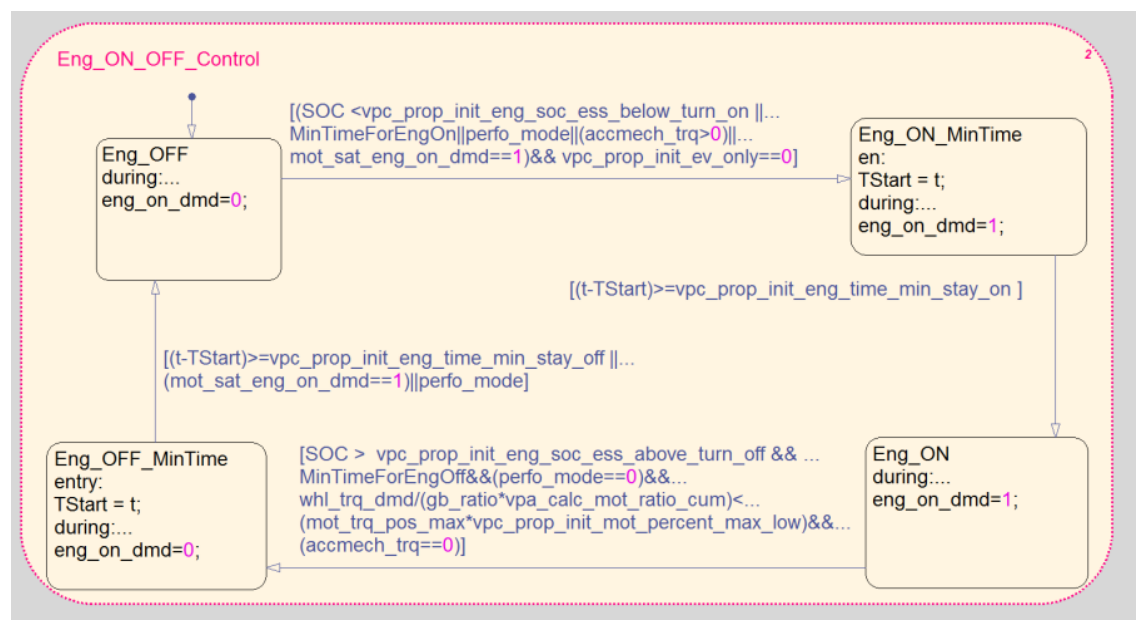

Figure 2.5 Thermostat control strategy for a series HEV (Source: Autonomie vRev12)

In power follower (baseline) control strategy, the ICE is the primary source of power, and the electric powertrain system provides the additional power or sustains a charge in the 
ESS. This controller is used in parallel and split powertrain hybrid vehicle, such as Honda Insight and Toyota Prius HEVs. The disadvantage is that the controller doesn't consider the powertrain component efficiency and the reduction in exhaust emissions (Rajamani 2011). The modified power follower strategy is an improved baseline control strategy, which is based on energy usage and emissions through a cost function representing overall FC and emissions at all candidate operating points. Although this strategy has helped to some extent in solving the problems associated with the former approach, repeating calculation of all candidates operating points is not desirable for online implementation (Johnson, Wipke and Rausen 2000). Another state machine based controller is composed of a set of operation modes, where mode selection is determined by considering a change in driver demand. This strategy cannot guarantee the vehicle will be able to obtain the optimized energy usage and emissions (Sorrentino, Rizzo and Arsie 2011).

\section{The Fuzzy Rule-Based Control Strategy}

Fuzzy logic is ideal for multi-domain, nonlinear, and time-varying hybrid electric powertrain system since it is robust, adaptable to variations, and easily adjustable. The fuzzy rule-based methods include conventional fuzzy strategy, fuzzy adaptive strategy and fuzzy predictive strategy (Wirasingha and Emadi 2011).

The inputs of the conventional fuzzy logic controllers are battery SOC and the desired ICE torque. Based on these inputs, the controller then selects a certain operation mode (Lee, et al. 2000). The application of this controller was shown to achieve better FE than the simple deterministic rule-based strategy. However, the conventional fuzzy logic controllers are still based on predetermined rules and can only be optimized for specific driving patterns. The fuzzy adaptive strategy is based on weight of fuel efficiency and emissions, where the weight defines the relative importance of these parameters. The main drawback of the fuzzy adaptive strategy is neglecting the driveline efficiencies (Schouten, Salman and 
Kheir 2002). Fuzzy predictive strategy was explored by Rajagopalan, et al. (2003), which is based on driving history and current data to decide the future operation modes through a look-ahead window along a fixed route. This controller can be implemented in real-time control tasks but still does not guarantee optimized vehicle performance.

\subsubsection{Optimization Based Control Strategies}

Rule-based control strategies only optimize the performance of each component individually, not maximize the efficiency of the powertrain, and so can't optimize the system efficiency of HEV. Optimization based control strategies can maximize the efficiency of the powertrain and minimize the cost function of FC or emissions (Amiri, et al. 2009). Optimization based controllers can be classified as global optimization or realtime optimization controllers.

\section{Global Optimization}

Global optimization, also known as horizon optimization, requires the knowledge of future and past power demands to minimize the cost function of FC and emissions over a fixed driving route. The controllers are often optimized offline and then implemented as an optimal performance benchmark of a HEVs' control strategies. Global optimization control strategies can be divided into linear programming, genetic algorithm (GA), DP and stochastic DP categories (Bayindir, Gözüküçük and Teke 2011).

Linear programming is mostly used for fuel efficiency optimization in series HEVs (Wirasingha and Emadi 2011). It uses piecewise linear approximations to transform hybrid powertrain, fuel efficiency and emissions into linear programs. The linear programming depends on powertrain structure. However, building a linear model for a complex topology is difficult. 
A genetic algorithm is easy to use for complex nonlinear optimization for the wide range of search space. However, unlike an analytical approach, the GA method needs more time for heavy calculations and can't provide the designer with the necessary view of HEV powertrain. Montazeri-Gh (2006) applied a GA for the optimization of the control parameters in a parallel $\mathrm{HEV}$, and the fitness function was defined to minimize the vehicle FC and emissions.

DP is both mathematical optimization and a computer programming method, which is one of the most popular approaches for solving the optimal power management problem of HEVs by minimizing a cost function over a driving cycle. DP can easily handle the constraints and nonlinearity of the problems while obtaining a globally optimal solution. The main drawbacks of the DP method include the computational complexity and the requirement of full knowledge of the drive cycle, which is not available prior to the completion of the actual vehicle mission. Therefore, DP is not able to adapt to any changes and cannot be utilized in a real-time controller (Bilbao, et al. 2014, Mansour and Clodic 2012, Sundstrom and Guzzella 2009).

The stochastic DP optimization method uses random variables to formulate an optimization problem, which serves to maximize its rewards in the worst-case scenario while providing the required torque demanded by the driver and keeping the SOC of the battery at a steady level. Lin (2004) found that the obtained stochastic DP control algorithm was better than a rule-based control strategy trained from deterministic DP results. This method still requires prior knowledge of the routes.

\section{Real-Time Optimization}

A global optimization algorithm can't be implemented directly online due to the pre-known driving cycle information and the cost of calculation. Instead, real-time optimization, which solves an optimization problem for only the current step, is implementable. Real- 
time optimization control strategies can be divided into Pontryagin's minimum principle (PMP), robust control approach, ECMS and A-ECMS.

PMP is a special case of Euler-Lagrange equation of calculus for variations. For an optimum solution, PMP provides only necessary conditions. The sufficient conditions are satisfied by Hamilton-Jacobi-Bellman equation. In PMP, the number of nonlinear secondorder differential equations linearly increases with the dimension. Control based on PMP usually takes less computational time for getting an optimal trajectory, so PMP is suggested as a viable real-time strategy (Kim, Cha and Peng 2011). Accordingly, PMP is still recognized as a local optimal, not a global solution.

The ECMS is developed by calculating the total FC as sum of real fuel consumed by the ICE and equivalent fuel consumption of the electrical system (Delprat, Guerra and Rimaux 2002, Paganelli, et al. 2002). The equivalent power consumption is expressed as $m_{f, e q u}=$ $m_{f}+\lambda \cdot P_{\text {ele }}$, where $m_{f, e q u}$ is the equivalent fuel consumption, $m_{f}$ is the fuel consumption by the ICE, $P_{e l e}$ is the electric energy consumption, and $\lambda$ is the equivalent factor that converts electrical energy into fuel consumption. This control method minimizes the equivalent fuel consumed by the ICE and battery. As shown in Figure 2.6, a small perturbation of $\lambda$ leads to non-charge sustaining operation. The red line (the optimal value of $\lambda=2.227$ ) is the sustaining SOC trajectories. Although ECMS is able to achieve nearly optimal performance, it is very sensitive to the variation in $\lambda$, and results in significant variation of final SOC. The operation of ECMS with $\lambda=3$ will charge the battery to the up-SOC limit, which makes it impossible to further store the electrical energy recovered from the braking process. However, ECMS operation with $\lambda=1$ would discharge to its low-SOC limit which makes it impossible to run the electric motor when high driving power is requested. 


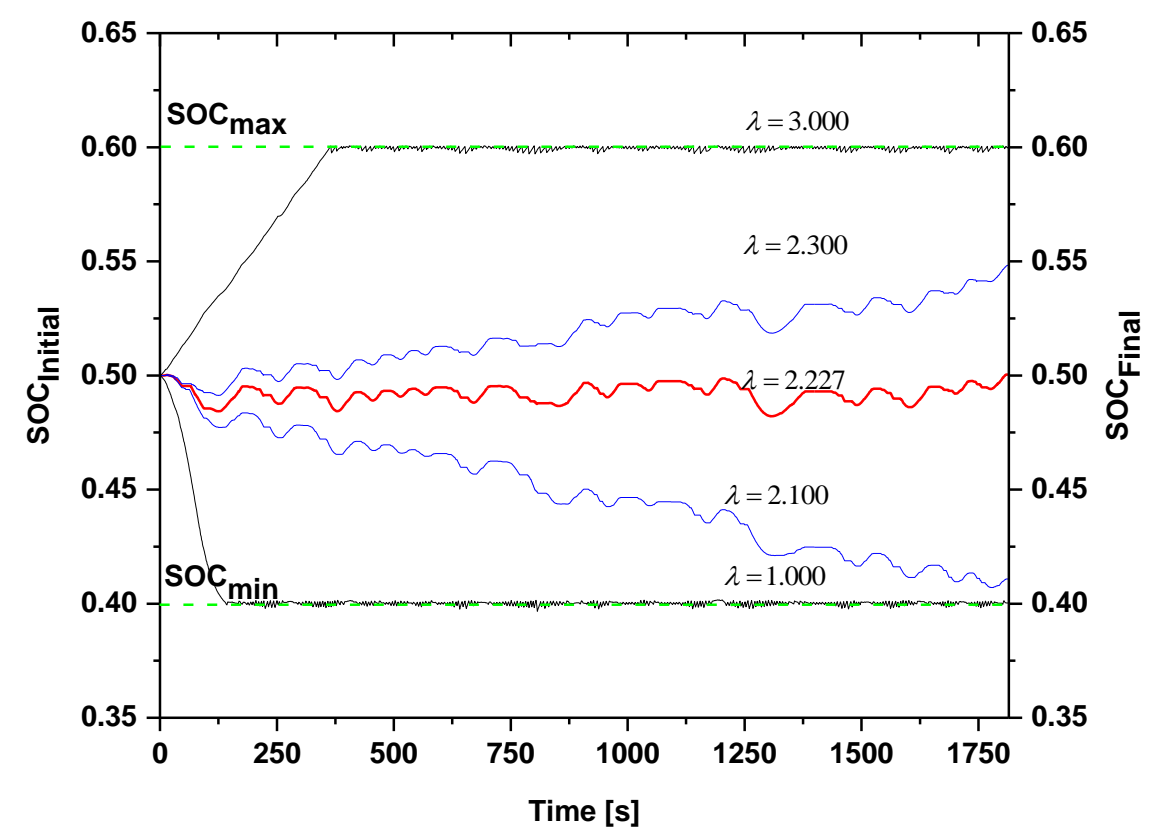

Figure 2.6 SOC trajectories with the variation of $\lambda$ (Beijing cycle)

Over-charging or depleting the battery can be solved by the A-ECMS, which adjusts the value of $\lambda$ based on the feedback from the current SOC (De Jager, Steinbuch and Keulen 2008, Musardo, et al. 2005). These methods actually account for the dynamic change in the value of $\lambda$ to maintain the SOC around the reference level. Although A-ECMS is easily utilized, robust and computationally cheap, it cannot guarantee near optimality. The reason for this is A-ECMS is extended by penalizing the deviation of SOC from a reference value. If the battery isn't sufficiently large, the ESS can't absorb all the energy made available from regenerative braking (Ambuhl and Guzzella 2009).

\subsubsection{Optimal Predictive Control Strategies}

As previously outlined, the SOC and power demand of the current driving state are the only inputs to both rule-based and optimization based control strategies, but these control strategies do not account for the upcoming route and vehicle operation information. In the case where the upcoming route information was not given, the engine was shut off during idle time and the lower power ranges with the driving power provided by the motor. The 
calculated SOC before the start of the high speed micro-trip was very low. In another case with the upcoming high speed micro-trip accounted for, the engine provides the electrical work to increase the SOC of the battery to its upper limit. With the upcoming route accounted for, the SOC of the battery is bumped to its high limit, which makes it possible to run the engine in its high efficiency region with partial power provided by the battery through avoiding the operation of the engine at extremely high load. Lin (2002), Cassebaum (2011), and Larsson (2014) stated that the optimal predictive control could offer superior fuel economy to the instantaneous strategies. The prediction model can be categorized into two approaches: model-based method and data-driven method (Shen 2008).

Model based approaches can predict future traffic conditions on the route of interest based on traffic flow theory. There are two different types of model based approaches: macroscopic and microscopic. Macroscopic models study traffic from an overall or average perspective in order to describe the traffic situation while microscopic models study the motion of individual vehicles (Miller 2011). These model based approaches are computationally complex and require expertise for design and maintenance of the traffic model.

Data driven approaches relate observed traffic conditions with current and past traffic data without requiring extensive expertise, so these approaches are fast and easy to implement (Park, et al. 2011). Various data driven prediction algorithms have been proposed to exploit driving conditions over the past several years including fuzzy logic, auto regressive integrated moving average (ARIMA), non-parametric regression, Kalman filter, Bayesian classifying, decision tree, fuzzy clustering analysis, neutral network (NN), support vector machine, and Markov chain (Xu, et al. 2009, Shen 2008, Miller 2011). Two levels of driving information can be used in optimal predictive control strategies. 


\section{The Past Driving Information of Route}

The past driving data can be collected, archived, calculated and analyzed by the data logger GPS unit (see Figure 2.7). The GPS is installed on the transit bus, and the on-board GPS \& communication module automatically sends the bus's running data to the operation center. The stored data were longitude, altitude, time stamp, differential fix, differential age, dilution of precision, etc. Some data from the vehicle, including vehicle speed, brakes and accelerations, are recorded. Those data reflect the real world environment, reflecting the actual road features, traffic situations, and driving behavior.

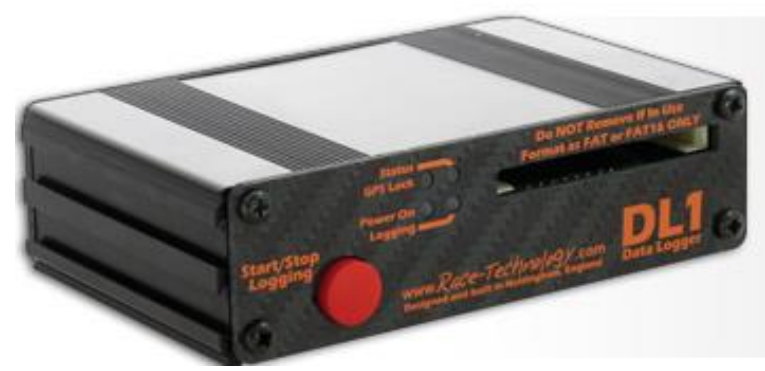

Figure 2.7 GPS and data logger unit

The stored history database can be used to develop a driving pattern prediction model, which can provide integrated driving information for the vehicle main controller to make decisions. The control strategy can be more adaptive to a variety of driving conditions (Lin, Jeon, et al. 2004, Xu, et al. 2009). There are numerous short-term prediction models, such as multiple-regression analysis, time-series forecasting, artificial neural networks and adjusted Kalman filter (Lee, Lee and Cho 2006). The past driving information is the critical point of predicting short term speeds. More complete and accurate past data will provide more precise predictions. 


\section{The Current Driving Information of the Route}

The geographic information system (GIS), GPS, intelligent transportation systems (ITS), vehicle to vehicle $(\mathrm{V} 2 \mathrm{~V})$ communication, and vehicle to infrastructure (V2I) create more opportunities to provide the current driving information with details such as time, speed, trip distance, slope, acceleration, and deceleration (C. Zhang 2010). Hajimiri (2006) designed a fuzzy logic controller with the driving condition difference between the current and predicted future condition accounted for. The short-term traffic properties prediction is based on the data driven technique and is a critical component for optimal predictive control system (Vlahogianni, Karlaftis and Golias 2014, Wang and Lukic 2011).The current driving information should be acquired online in a certain time or distance based segment. If the segment is too short, the current data may not reflect the driving cycle correctly; if the segment is too large, the computational burden may be too heavy for realtime control (Ye, Szeto and Wong 2012, Chan, Dillon and Chang 2013).

\subsection{Summary}

Figure 2.8 summarizes the control strategies of hybrid vehicles. This serves as a guide to identify or select the correct method of optimization. It is suggested that the best strategy should be implemented in real-time, provide global optimal results, and get fit to the dynamic simulation environment. In my research, a model which is capable of predicting the forgoing vehicle driving patterns and combines the advantages of both DP and ECMS method will be developed. 


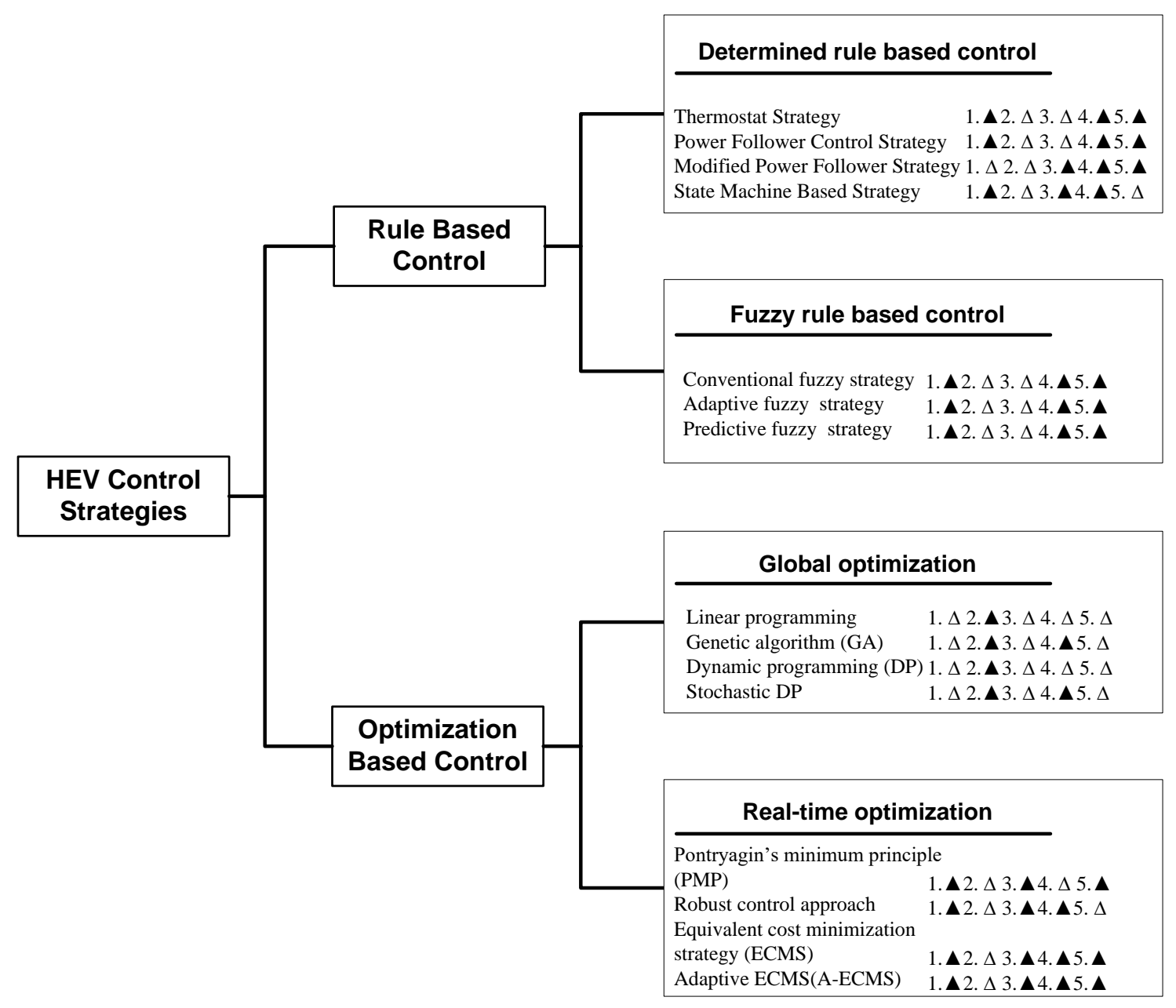

Note: Benefit of controller: 1. Real-time controller; 2. Global optimal solution; 3. Local optimal solution; 4. Nonrequirement of priori knowledge of route; 5. Structure simplicity. ( $\Delta$ means Possess, $\Delta$ means Not Possess)

\section{Figure 2.8 Classification and comparison of control strategies}




\section{Hybrid Electric Vehicle Modeling and Validation}

\subsection{Modeling of the Hybrid Electric Powertrain}

The parallel hybrid bus powertrain model is based on a transit bus equipped with a pretransmission parallel hybrid powertrain and a 4.76L Deutz-BF4M1013FC diesel engine. Table 3.1 presents the specifications of the parallel hybrid bus. The topology of the full parallel hybrid electric powertrain is showed in Figure 3.1.

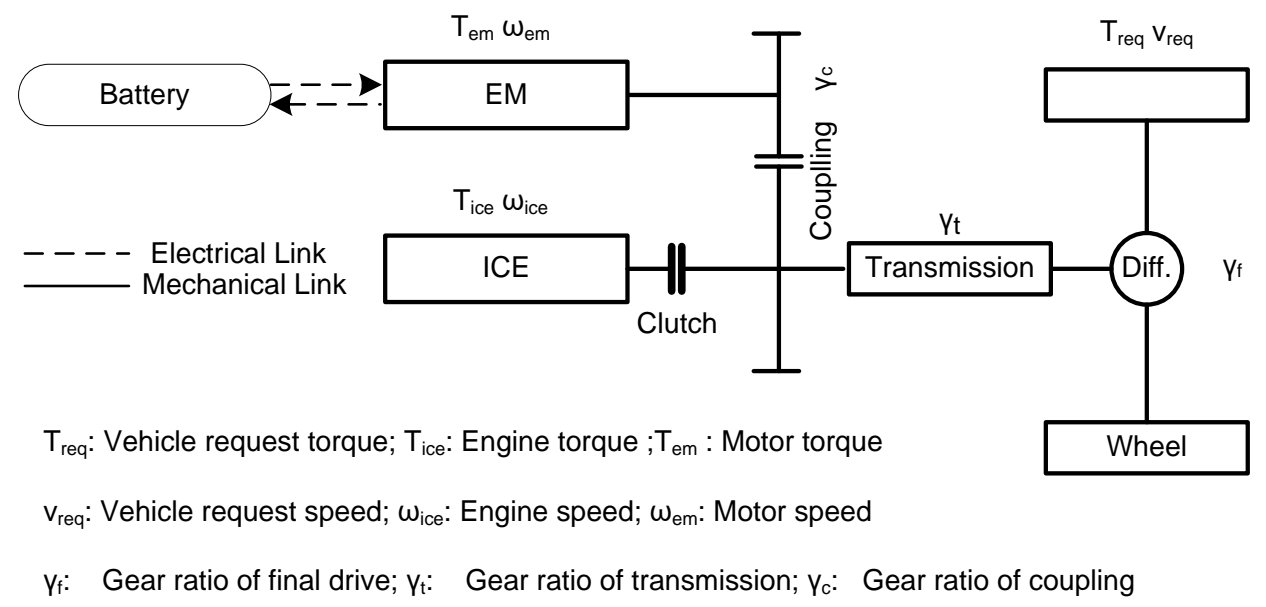

Figure 3.1 Topology of the full parallel hybrid electric powertrain

The model accounts for vehicle longitudinal dynamics, and has static nonlinear maps describing the efficiency of the combustion engine, electric machine, and battery.

\subsubsection{Engine Model}

The engine of this transit bus is an electronically controlled in-line 4 cylinder 4.76L Deutz diesel engine equipped with an intercooler and turbocharger. The rated power is $140 \mathrm{~kW}$ at $2300 \mathrm{rpm}$ and the peak torque is $646 \mathrm{Nm}$ at $1400 \mathrm{rpm}$ from the test data. 
Table 3.1 Basic specifications of the parallel hybrid transit bus (Zeng 2005)

\begin{tabular}{|c|c|}
\hline Component & Specifications \\
\hline Engine & $\begin{array}{l}\text { Model: Deutz-BF4M1013FC } \\
\text { No. Cylinders: } 4 \\
\text { Air Intake: Turbocharged } \\
\text { Power: } 140 \mathrm{~kW}(187 \mathrm{hp}) @ 2300 \mathrm{rpm} \\
\text { Torque: } 640 \mathrm{Nm}(472 \mathrm{ft}-1 \mathrm{~b}) @ 1600 \mathrm{rpm} \\
\text { Idle speed: } 900 \mathrm{rpm} \\
\text { Weight: } 560 \mathrm{~kg}\end{array}$ \\
\hline Motor/Generator $(M / G)$ & $\begin{array}{l}\text { Type: AC } \\
\text { Peak Power: 80kw (107hp) } \\
\text { Continuous Power: } 40 \mathrm{kw}(53 \mathrm{hp}) \\
\text { Peak Torque: } \pm 380 \mathrm{Nm}(280 \mathrm{ft}-\mathrm{lb}) \\
\text { Continuous Torque: } \pm 260 \mathrm{Nm}(191 \mathrm{ft}-\mathrm{lb}) \\
\text { Base Speed: } 1500 \mathrm{rpm} \\
\text { Maximum Speed: } 5000 \mathrm{rpm} \\
\text { Voltage: } 360 \mathrm{~V}\end{array}$ \\
\hline Energy storage & $\begin{array}{l}\text { Type: nickel-metal hydride battery (NiMH) } \\
\text { Single Voltage: } 12 \mathrm{~V} \\
\text { Cell No.: } 30 \\
\text { Capacity: } 27 \mathrm{Ah} \\
\text { Peak power(estimate): } 75 \mathrm{~kW}(100 \mathrm{hp}) \\
\text { Weight(estimate): } 174 \mathrm{~kg}\end{array}$ \\
\hline Drivetrain & $\begin{array}{l}\text { Type: 6-speed AMT } \\
1^{\text {st }} \text { gear ratio: } 7.285 ; 2^{\text {st }} \text { gear ratio: } 4.193 \\
3^{\text {st }} \text { gear ratio: } 2.485 ; 4^{\text {st }} \text { gear ratio: } 1.563 \\
5^{\text {st }} \text { gear ratio: } 1.000 ; 6^{\text {st }} \text { gear ratio: } 0.847 \\
\text { Ratio of final drive: } 6.333\end{array}$ \\
\hline Vehicle & $\begin{array}{l}\text { Model: FAW-CA6110HEV } \\
\text { Dimensions: } 11496 \mathrm{~mm}(37.7 \mathrm{ft}) \times 2500 \mathrm{~mm}(8.2 \mathrm{ft}) \times 3075 \mathrm{~mm}(10.1 \mathrm{ft}) \\
\text { Overall Weight: } 15000 \mathrm{~kg}(33069 \mathrm{lb}) \\
\text { Frontal Area: } 6.5 \mathrm{~m}^{2}(70 \mathrm{ft} 2) \\
\text { Aerodynamic Drag Coefficient: } 0.65 \\
\text { Coefficient of Rolling Resistance: } 0.0098 \\
\text { Wheel Radius: } 509 \mathrm{~mm}(1.67 \mathrm{ft})\end{array}$ \\
\hline
\end{tabular}

Calculation of fuel and $\mathrm{NO}_{\mathrm{x}}$ rate is based on the brake specific fuel consumption (BSFC) map and $\mathrm{NO}_{\mathrm{x}}$ emissions map, which are functions of engine speed $\omega_{\text {ice }}$ and torque $T_{\text {ice }}$.

$$
\dot{m}_{f u e l / N O_{x}}=f_{B S F C / N O_{x}}\left(\omega_{i c e}, T_{i c e}\right) \quad \text { Equation } 3.1
$$

The BSFC map and steady-state $\mathrm{NO}_{\mathrm{x}}$ emissions map for the engine are showed in Figure 3.2 and Figure 3.3, respectively. 


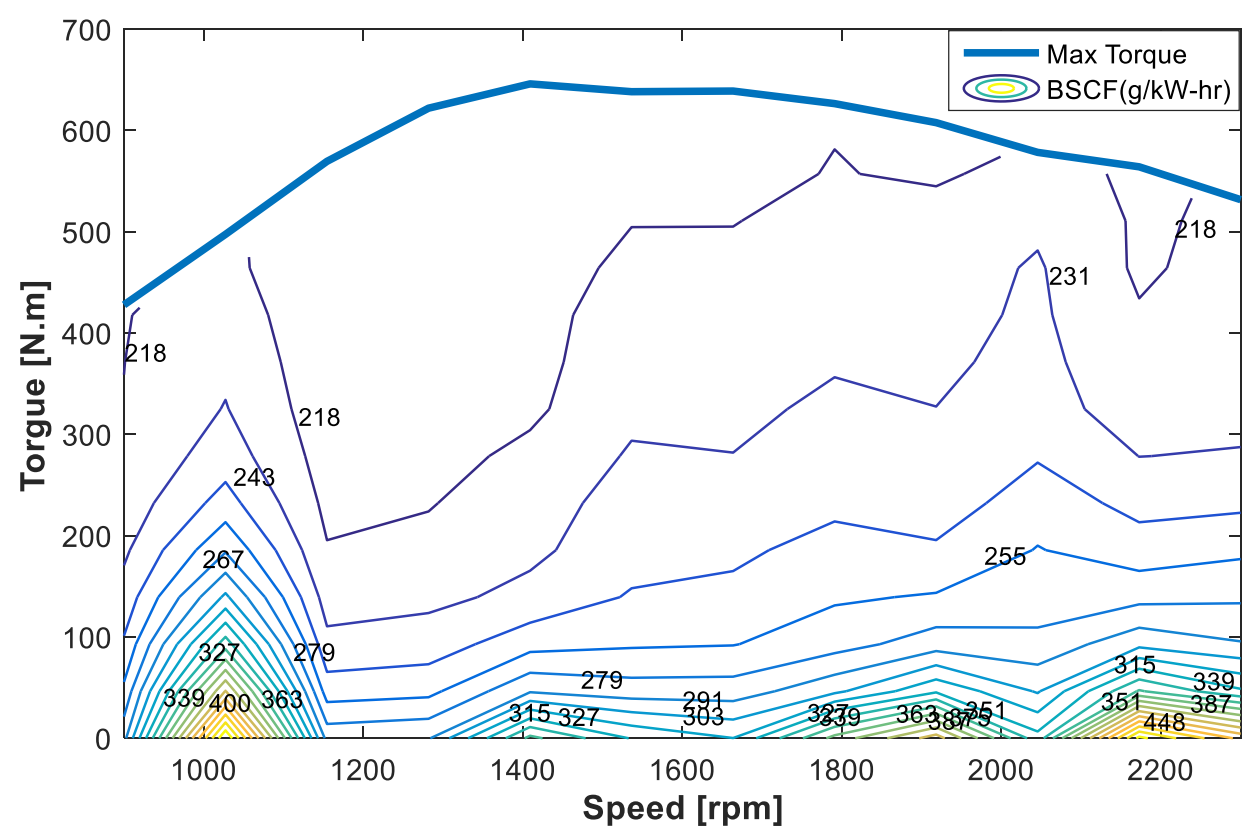

Figure 3.2 BSFC map (data from Chen 2010)

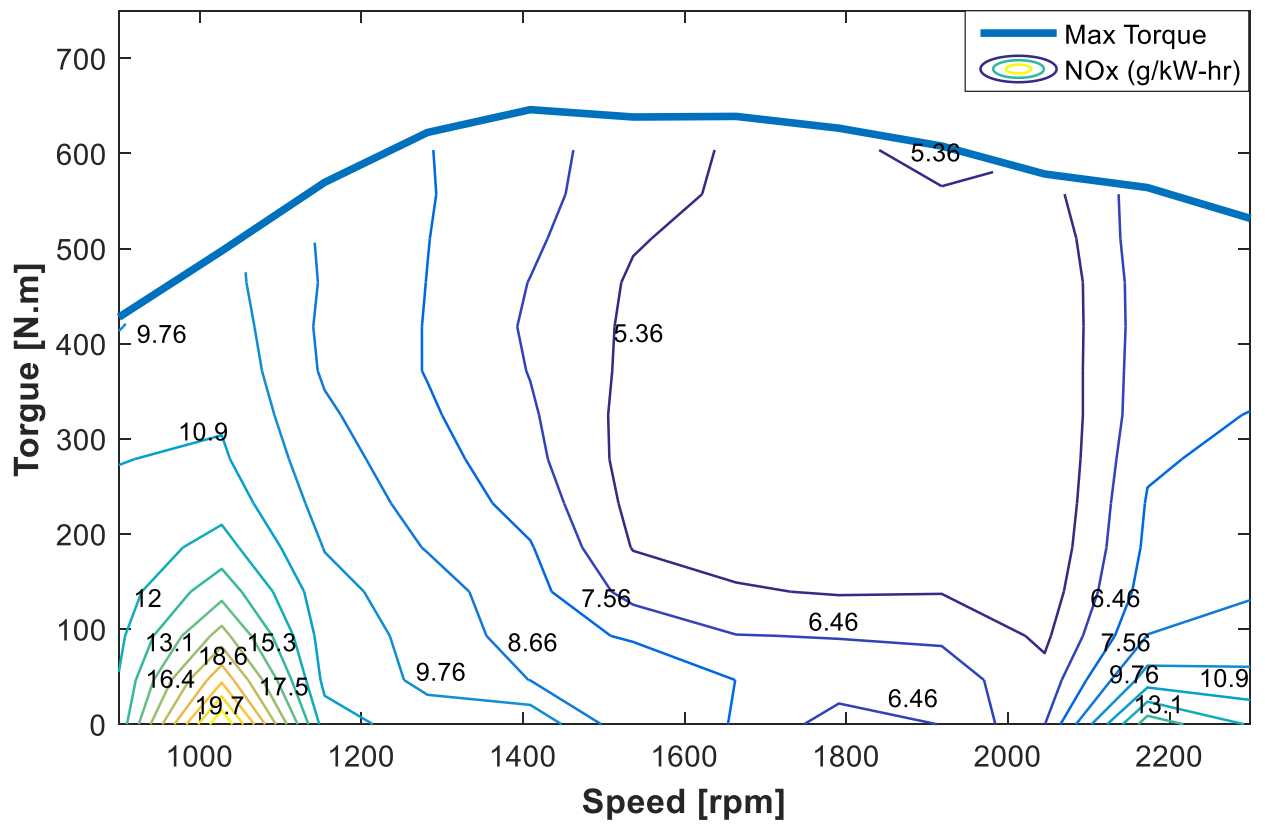

Figure 3.3 NO ${ }_{\mathrm{x}}$ emissions map (data from Chen 2010)

\subsubsection{Electric Machine Model}

The performance of the electric machine (EM) can be represented with the maximum torque, power, and speed. In this study, the EM model includes an AC asynchronous motor with a continuous power output of $40 \mathrm{~kW}$ and a peak power of $80 \mathrm{~kW}$, an inverter, and an 
EM controller. The maximum torque is $\pm 260 \mathrm{Nm}$, the rotating inertia of the rotor is assumed to be $0.047 \mathrm{~kg} \cdot \mathrm{m}^{2}$ (Chen 2010).

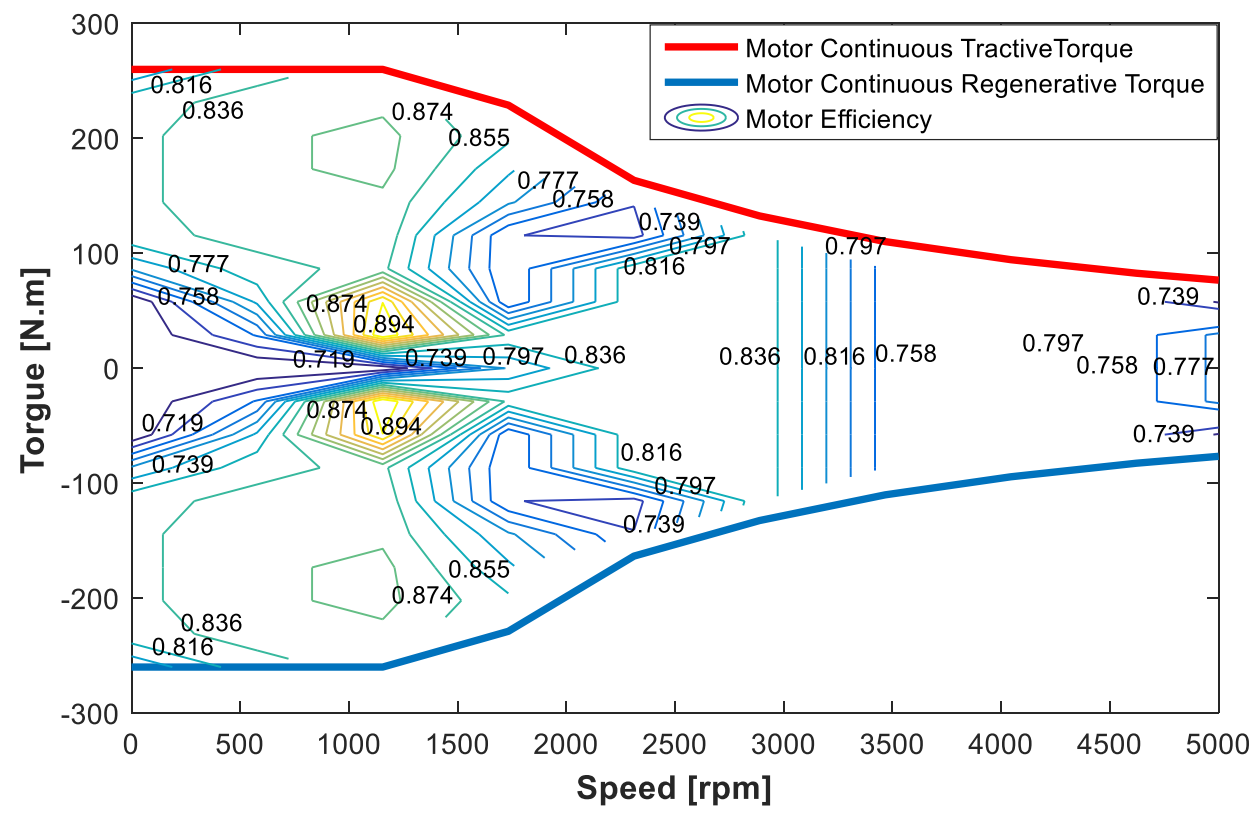

Figure 3.4 Efficiency map of the EM (data from Chen 2010)

Equation 3.2 and Equation 3.3 describe the relationships between the inputs and outputs of the EM model.

$$
\begin{array}{cr}
T_{\text {em }}=\operatorname{sign}\left(T_{\text {em,req }}\right) \cdot\left|\min \left(T_{\text {em,req }}, T_{\text {em,max }}, T_{\text {batt,max }}\right)\right| & \text { Equation } 3.2 \\
P_{\text {em }}=T_{e m} \omega_{e m}\left(\eta_{\text {em }}\right)^{k} & \text { Equation } 3.3
\end{array}
$$

Where $T_{e m, r e q}$ is the requested EM torque, $T_{e m, \max }$ is the maximum torque limit posed by the EM, $T_{\text {batt,max }}$ is the maximum torque limit posed by the battery, $P_{e m}$ is the required electrical power, and $\eta_{e m}$ is the EM efficiency as a function of torque and speed, as shown in Figure 3.4. $k$ indicates the direction of the power flow. If the EM is used as a motor, $k=-1$ and the motor provides propulsion power to the vehicle. When regenerating, $k=$ 1 and the vehicle's kinetic energy is converted to electrical energy and then stored in the battery. 


\subsubsection{Battery Model}

The battery used is a NiMH battery with a capacity of 27 Ah. The battery model was developed based on a simple resistive equivalent circuit model, where the open-circuit voltage $V_{o c}$ and resistor $R_{i}$ are functions of battery SOC at $40{ }^{\circ} \mathrm{C}$ as shown in Figure 3.5 and Figure 3.6, respectively. This simple model allows for the expression of the terminal voltage of the battery as

$$
V_{b}=V_{o c}-R_{i} \cdot I_{b}
$$

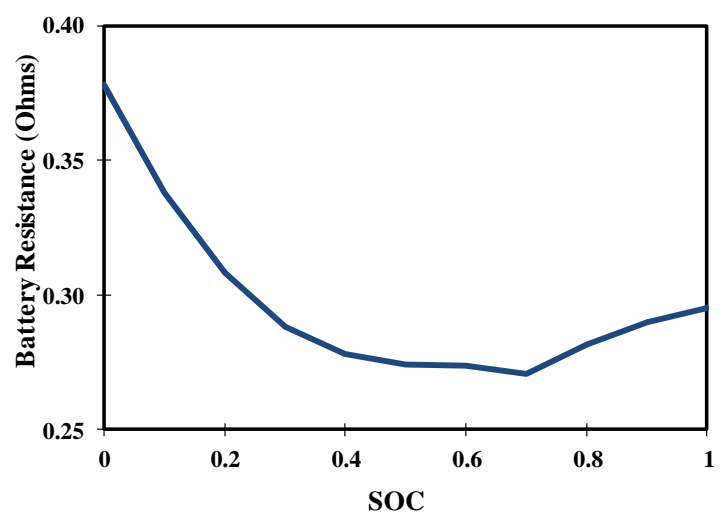

Figure 3.5 Internal resistance of the NiMH battery as a function of SOC at $40{ }^{\circ} \mathrm{C}(\mathrm{Chen}$ 2010)

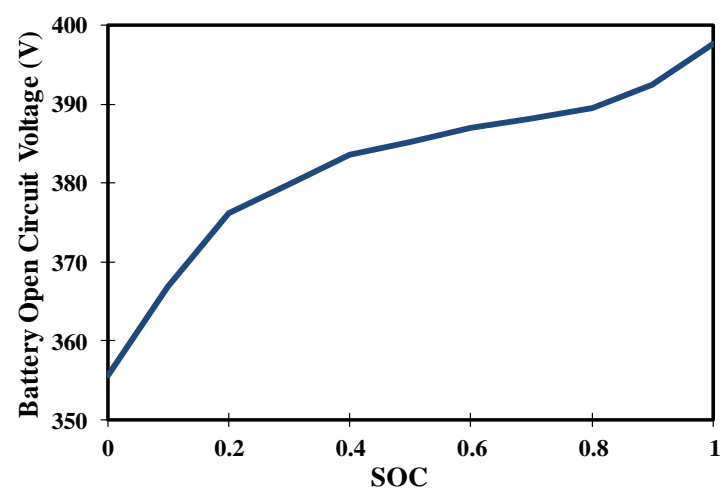

Figure 3.6 Internal OCV of the NiMH battery as a function of SOC at $40{ }^{\circ} \mathrm{C}$ (Chen 2010) The requested battery power $\left(P_{b}\right)$ is

$$
P_{b}=V_{b} \cdot I_{b}=V_{o c} \cdot I_{b}-R_{i} \cdot I_{b}^{2}
$$


The battery current is calculated from the above requested battery power $\left(P_{b}\right)$ equation.

$$
I_{b}=\frac{V_{o c}-\sqrt{V_{o c}^{2}-4 \cdot R_{i} \cdot P_{b}}}{2 \cdot R_{i}} \quad \text { Equation } 3.6
$$

By the Coulomb counting method, the SOC of the battery is

$$
\operatorname{SOC}(k+1)=\operatorname{SOC}(k)-\frac{\int_{k_{0}}^{k_{f}}\left(\frac{V_{o c}-\sqrt{V_{o c}^{2}-4 \cdot R_{i} \cdot P_{b}}}{2 \cdot R_{i}}\right)}{Q_{b}} \quad \text { Equation } 3.7
$$

The parameter $Q_{b}$ is the nominal battery capacity. The SOC of the battery is physically limited to $[0,1]$.

\subsubsection{Transmission Model and Torque Coupler Model}

The transmission adjusts engine speed $\omega_{i c e}$ and torque $T_{i c e}$ to meet the wheel power demand $P_{r e q}$ while keeping the engine from operating too aggressively. A complete gearbox model includes two critical maps: a gear ratio map, which is indexed by gear position, and a transmission efficiency map, which is a function of speed, torque and gear position. The test transit bus was equipped with a 6 speed automated manual transmission which combined the advantages of manual transmission, such as light weight and high efficiency, and the advantages of automatic transmission, such as convenience for drivers. It had the potential to improve efficiency by up to $7 \%$ at no cost to driving comfort (Zeng 2005). The transmission ratios were 7.285 (1st gear), 4.193 (2nd gear), 2.485 (3rd gear), 1.563 (4th gear), 1.000 (5th or direct gear), and 0.847 (6th gear).

A torque coupler is a device with a fixed ratio installed between the motor and the transmission, by which motor speed and torque are divided and multiplied, respectively. 


\subsubsection{Chassis Model}

The chassis model was based on the road load equation shown in Equation 3.8, which considered rolling resistance, aerodynamic drag, road grade, and acceleration loss related to inertia and mass of the powertrain.

$$
\begin{aligned}
P_{\text {req }}=P_{r}+P_{w d} & +P_{g d}+P_{a c c} \\
& =\frac{1}{3600 \cdot \gamma_{t}}\left(m \cdot g \cdot f \cdot \cos \alpha+\frac{C_{D} \cdot A \cdot v_{r e q}^{2}}{21.15}\right. \\
+ & \left.m \cdot g \cdot \sin \alpha+\sigma \cdot m \cdot \frac{d_{v_{r e q}}}{d_{t}}\right) \cdot v_{r e q}
\end{aligned}
$$

Where $\gamma_{c}, \gamma_{t}, \gamma_{f}$ and $\gamma_{t}=\gamma_{c} \cdot \gamma_{t} \cdot \gamma_{f}$, are the torque coupler efficiency, gearbox efficiency, final drive efficiency and overall transmission efficiency, respectively. The $\alpha=\tan ^{-1}(i)$ denotes grade angle. $P_{r}, P_{w d}, P_{g d}$ and $P_{a c c}$ represent power losses due to the rolling resistance, wind drag, climbing, and the power needed for acceleration, respectively. The $\sigma$ represents a mass coefficient with inertia effect accounted for.

\subsubsection{The Rule-Based Control Strategy}

In the forward-looking simulation tool Autonomie, the control strategy is the simplest determined rule-based controller (Slezak 2013). It operates on the simple principle that the ICE will be turned on or off based on the SOC and the torque request of the vehicle. The controller has been used in series hybrid powertrains, where the EM provides all the propulsion, and the ICE is required to sustain a defined SOC. Rule-based control strategy is robust, simple and easy to implement but leads to high frequency on and off of the engine and charging and discharging of the battery.

Under the assumption that the power requirement can be satisfied, the rule-based control strategy of a parallel hybrid powertrain model should enable the SOC to be restored to its target quickly, which will allow for highly efficient charging and discharging of the battery 
and ensure the ESS has enough power to meet the powertrain requirement. When the SOC is above the maximum threshold allowed for the ESS, the strategy will switch to a discharging mode. In this mode, the vehicle will be propelled mainly by the electric motor if possible. The engine will not be turned on unless the torque demand from the vehicle is above the maximum torque of the motor and vehicle speed is above the electric launch speed. When the SOC is below its minimum value, the strategy will switch to the recharging mode. The engine will provide excess power to recharge the ESS through the electric generator.

\subsection{Validation of Hybrid Bus Model}

The parallel hybrid bus powertrain simulation model is developed referring to a CA6110HEV transit bus, which was tested and reported by Zeng (2005) using the China's Urban Bus (CUB) cycle and the Beijing cycle with different initial SOCs. Chen (2010) developed and validated one simulation model using PSAT.

In this research, Autonomie's composite modeling and simulation tool was utilized to develop and validate the transit bus model. The simulation results were compared with the fuel consumption measured by Zeng (2005) when the initial SOC in each simulation was set identical to that in the test.

Figure 3.7 and Figure 3.8 show the measured versus simulated vehicle speed and simulated SOC for the parallel hybrid bus model on the Beijing driving cycle, respectively. The initial SOC is $51 \%$. Figure 3.9 shows the simulated engine and motor operation under the rulebased controller. From Figure 3.9 (b), the motor in the hybrid bus mostly charge the battery with electricity recovery during the braking process or the engine power. As shown in Figure 3.9 (a), the engine propels the vehicle with low torque throughout the cycle, so there is no pure electric mode in the simulation results. 


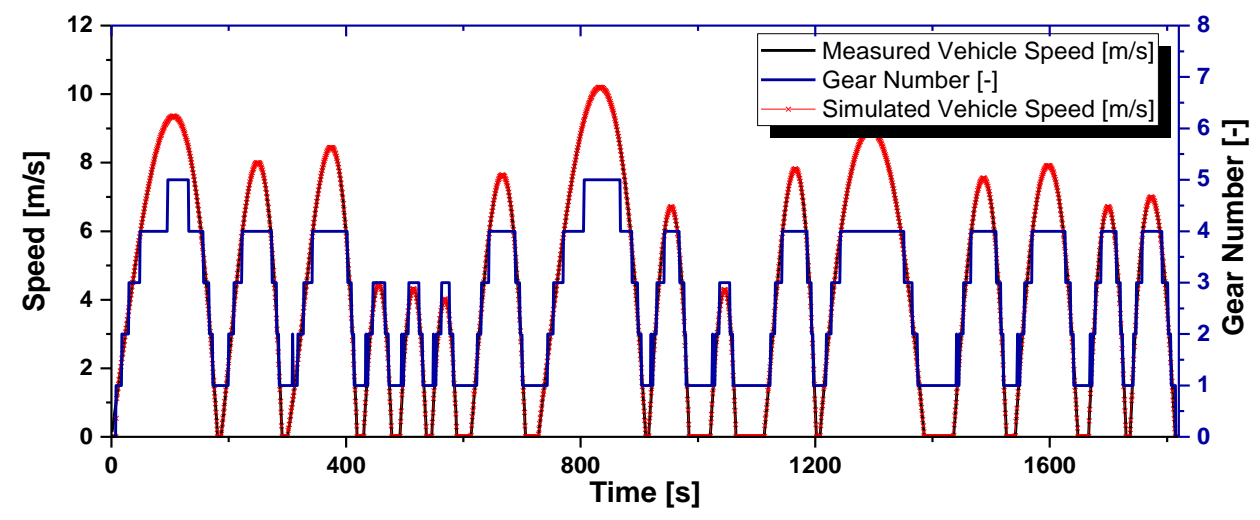

Figure 3.7 Measured versus simulated vehicle speed and gear number over the Beijing cycle

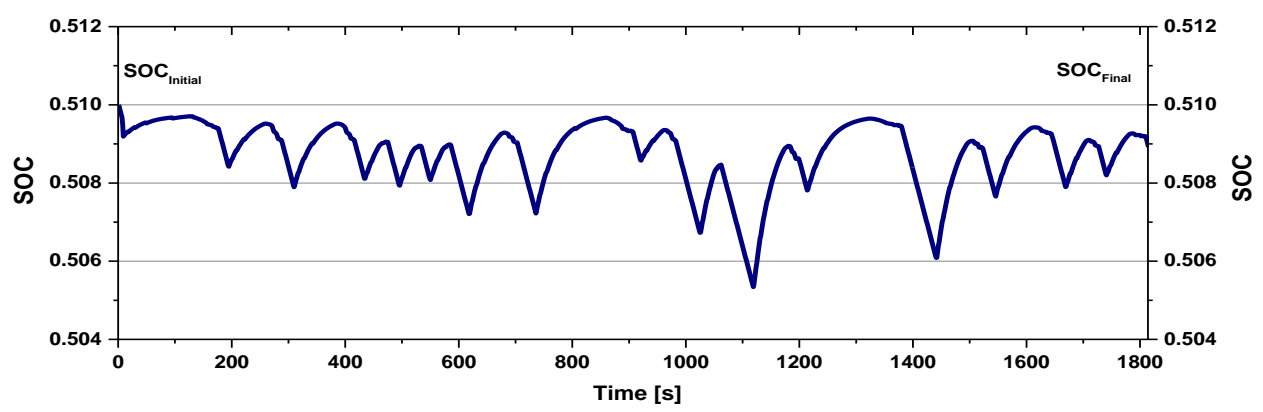

Figure 3.8 Simulation of SOC over the Beijing cycle (Initial SOC=51\%)

The model was validated by comparing the fuel consumption and the change in SOC ( $\triangle$ SOC). Table 3.2 shows the fuel consumption and battery SOC measured by Zeng (2005), simulated using PSAT (Chen 2010), and Autonomie in this research, respectively. The fuel consumption and relative deviation predicted was consistent with experimental data with an error of less than $5 \%$. The vehicle model is considered validated. 


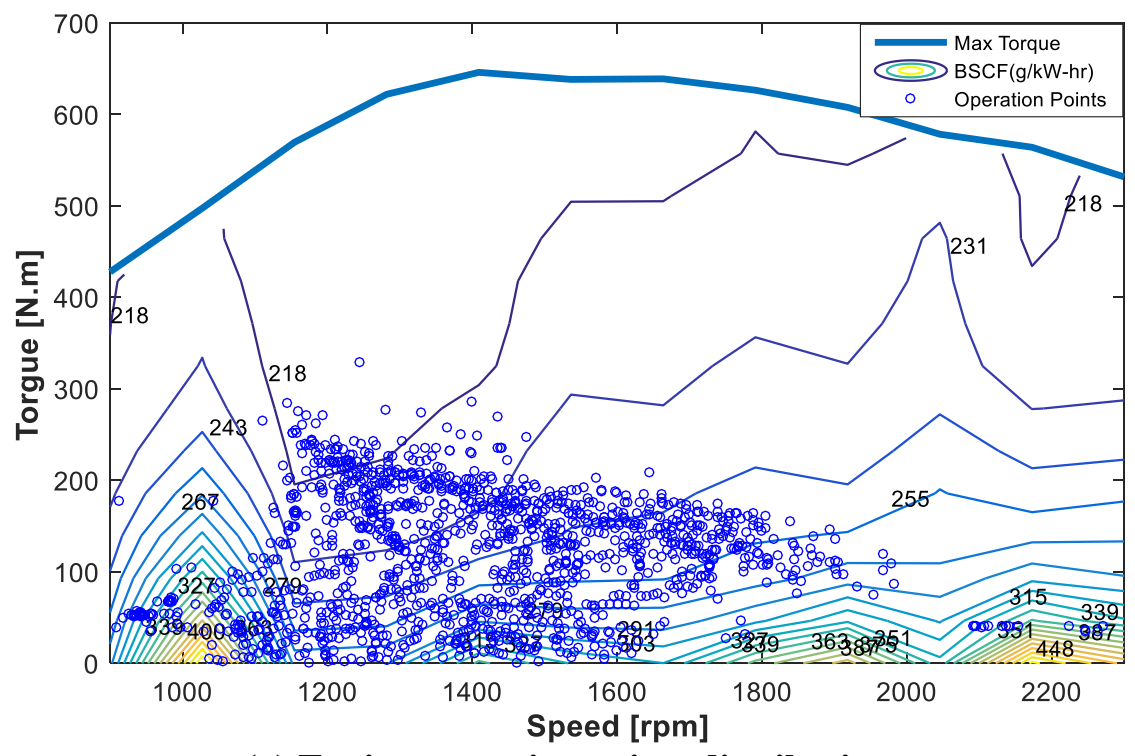

(a) Engine operating points distribution

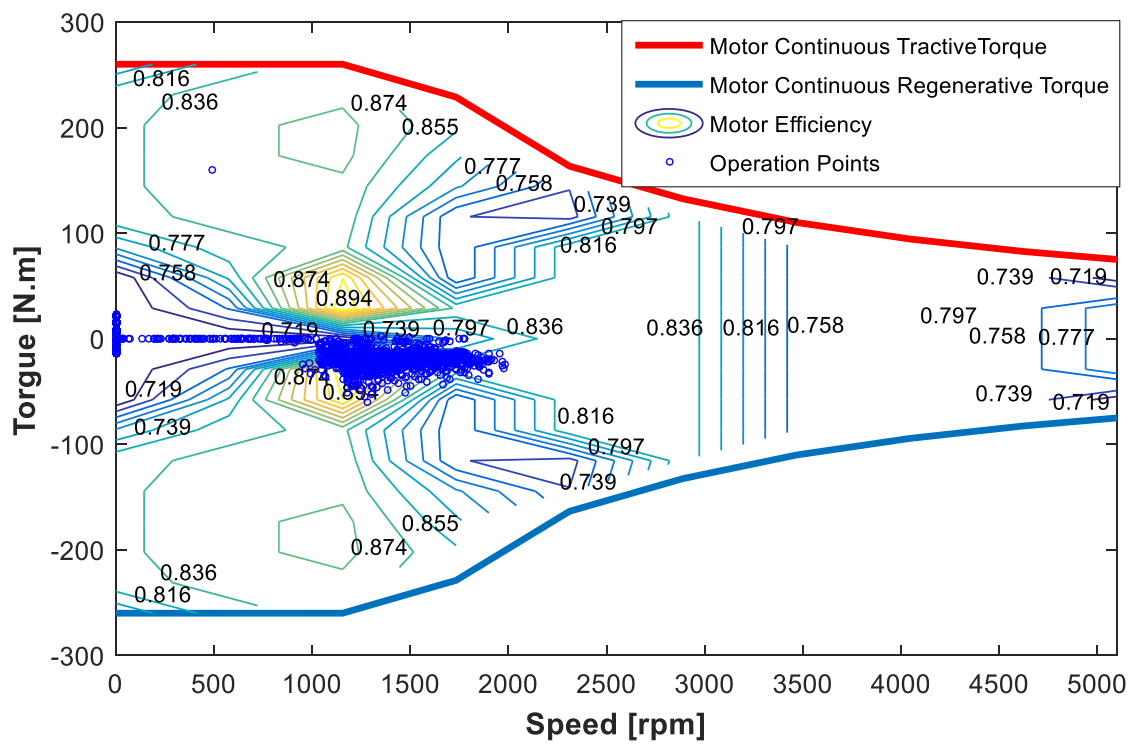

(b) Motor operating points distribution

Figure 3.9 Distribution of power source operating points over the Beijing cycle 
Table 3.2 Validation results of the hybrid bus model (Zeng 2005, Chen 2010)

\begin{tabular}{|c|c|c|c|c|c|c|}
\hline \multirow[b]{2}{*}{ Cycle } & \multirow[b]{2}{*}{ Data source } & \multicolumn{2}{|c|}{ Fuel consumption } & \multirow{2}{*}{$\begin{array}{c}\text { Initial SOC } \\
\text { Value }(\%)\end{array}$} & \multicolumn{2}{|c|}{ Final SOC } \\
\hline & & $(\mathrm{L} / 100 \mathrm{~km})$ & $\begin{array}{c}\text { Relative } \\
\text { error }(\%)\end{array}$ & & Value $(\%)$ & $\begin{array}{c}\text { Relative } \\
\text { deviation }(\%)\end{array}$ \\
\hline \multirow{3}{*}{ CUB-5* } & Measured & 33.3 & - & \multirow{3}{*}{65.0} & 64.0 & - \\
\hline & PSAT simulated & 31.9 & 4.1 & & 66.7 & 4.2 \\
\hline & Autonomie simulated & 32.3 & 3.0 & & 64.8 & 1.2 \\
\hline \multirow{3}{*}{ CUB-5** } & Measured & 30.5 & - & \multirow{3}{*}{69.0} & 68.0 & - \\
\hline & PSAT simulated & 29.8 & 2.2 & & 66.9 & 1.6 \\
\hline & Autonomie simulated & 30.9 & 1.3 & & 68.7 & 1.0 \\
\hline \multirow{3}{*}{ CUB-10 } & Measured & 29.5 & - & \multirow{3}{*}{70.0} & 69.0 & - \\
\hline & PSAT simulated & 30.1 & 1.8 & & 66.8 & 3.2 \\
\hline & Autonomie simulated & 30.6 & 3.7 & & 69.8 & 1.2 \\
\hline \multirow{3}{*}{ Beijing } & Measured & 33.0 & - & \multirow{3}{*}{51.0} & 50.0 & - \\
\hline & PSAT simulated & 33.0 & 0.0 & & 51.6 & 3.2 \\
\hline & Autonomie simulated & 33.4 & 1.2 & & 50.9 & 1.8 \\
\hline
\end{tabular}

* is the five repeat CUB cycle with initial SOC of 0.65 ; ** is the five repeat CUB cycle with initial SOC of 0.69 .

\subsection{Summary}

Model assumptions and a description of the various elements of the hybrid electric transit bus model have been presented. Autonomie simulated results were compared with the experimental data over four various driving schedules. The validation results show that the relative errors were less than $4.1 \%$ and $4.2 \%$ for fuel consumption and the change in SOC, respectively. In conclusion, the parallel hybrid bus powertrain model is validated. The validated hybrid bus model will be used as a platform to simulate the effect of control strategies on fuel consumption and exhaust emissions. 


\section{DP and ECMS}

Rule-based control strategies only optimize the performance of each component individually, not the system efficiency of the powertrain. Optimization based energy management strategies can maximize the efficiency of the powertrain and minimize the cost function of fuel consumption or emissions. Optimization based controllers can be divided into global optimization and real-time local optimization.

\subsection{Dynamic Programming}

DP is a method for solving complex optimization problems by breaking them down into simpler sub-problems based on Bellman's principle of optimality (Bilbao, et al. 2014). It is a powerful tool for finding the global optimal solution of a nonlinear dynamic system for given boundary conditions.

The discrete formulation of DP is expressed as

$$
x(k+1)=f(x(k), u(k))
$$

Equation 4.1

Where $k$ is the discrete time index, $x(k)$ is the state vector, $u(k)$ is the control vector and $f(\cdot, \cdot)$ is a nonlinear, time-varying, discrete time mapping of the state variable and control input. To generate the best control vector $u(k)$, the principle of optimality is utilized to minimize or maximize the functional cost, which can be described by:

$$
J=S\left(x\left(k_{f}\right), k_{f}\right)+\sum_{k=0}^{k_{f-1}} V(x(k), u(k))
$$

Equation 4.2

In Equation 4.2, $J$ represents the minimized/maximized cost-to-go function, $S\left(x\left(k_{f}\right), k_{f}\right)$ is the cost function of the final state and $\sum_{k=0}^{k_{f-1}} V(x(k), u(k))$ is the sum of cost-to-go from every stage to the next stage as a function of the current state and control. In a backward DP algorithm, the last stage is solved first, and then the sub-problem involving last two stages, last three stages, etc. until the entire problem is solved step by step. The optimal 
cost-to-go is the cost-to-go from the current state to the final state when the minimized optimal control policy is applied as

$$
\begin{array}{cc}
J_{N}\left(k_{f}\right)=S\left(k_{f}\right) & \text { Equation } 4.3 \\
J_{k}(x(k))=\min _{u(k)}\left[V(x(k), u(k))+J_{k+1}(x(k+1))\right] & \text { Equation } 4.4 \\
k=N-1, N-2, \ldots, 0 &
\end{array}
$$

The DP algorithm can be implemented proceeding backward in time from step $N-1$ to step 0 , so if one had found the optimal control, state and cost from any stage $\mathrm{k}+1$ to the final stage $k_{f}$, then one can find the optimal values for any stage $k$ to the end stage $k_{f}$. The optimization goal is to find the control state $u(k)$ which minimizes the FC or emissions along a known in advance driving cycle.

\subsubsection{The Application of DP in Developing Hybrid Vehicle Control Strategies}

DP is frequently applied to hybrid vehicles to minimize the FC, and is accomplished using a backward approach and needs prior knowledge of the driving cycle. Firstly, the state/control variables, grid selection, constrains and cost matrix should be defined and explained.

\section{State Variables and Control Variables}

The state vector consists of the requested vehicle speed $v_{r e q}$, SOC, engine speed $\omega_{\text {ice }}$, and electric motor speed $\omega_{e m}$. The control vector includes engine torque $T_{i c e}$ and motor torque $T_{e m}$. In a parallel hybrid electric vehicle, some of the state and control variables depend on other state and control variables: $\omega_{i c e}=\frac{\omega_{e m}}{\lambda_{c}}=\frac{\omega_{r e q}}{\lambda_{f} \cdot \lambda_{t}}, T_{i c e}=T_{r e q}-T_{e m}$. Accordingly, the DP algorithm can be simplified into one control variable $\left(T_{\text {ice }}\right)$, and one state variable (SOC) while maintaining the accuracy of the solutions. 


\section{The Grid Selection}

The DP algorithm is based on a discrete decision route. Hence, the state and control variables have to be limited and discretized in the grids. Three grids are defined in this problem: the time grid $N \in\left[k_{0}, k_{0}+1, \cdots, k_{f}\right]$, control grid $\mathrm{M} \in\left[T_{i c e \_m i n}, T_{i c e \_m i n}+\right.$ $\left.1, \cdots, T_{\text {ice_max }}\right]$, and state grid $L \in\left[S O C_{1}, S O C_{2}, \cdots, S O C_{2000}\right]$.

The time is sampled in $N$ steps over the length of the driving cycle $k_{0}$ to $k_{f}$, where $N=$ $\frac{k_{f}-k_{0}}{k}$. The time step $k$ is 1 second during computation, so the length of time vector $N$ is the number of seconds of the whole route. The control grid $\mathrm{M}$ varies between the maximum $T_{i c e \_m a x}$ and minimum $T_{i c e \_m i n}$ engine torque, where $\mathrm{M}=\frac{T_{i c e \_m a x}-T_{i c e \_m i n}}{d\left(T_{i c e}\right)}$, and the step size of the control variable $d\left(T_{i c e}\right)$ is $1 \mathrm{Nm}$. The engine brake torque $T_{i c e \_m i n}$ is $-100 \mathrm{Nm}$. The maximum engine torque $T_{\text {ice_max }}$ is derived from the engine map function based on engine speed $\omega_{i c e}$ as described by Equation 4.5:

$$
T_{\text {ice_max }}(k)=f_{\text {engine } \operatorname{map}}\left(\omega_{\text {ice }}(k)\right)
$$

Equation 4.5

The state grid $L$ is $\frac{S O C_{\max }-S O C_{\min }}{d(\mathrm{SOC})}$ steps between the minimum and maximum SOC value. The initial $\mathrm{SOC}$ is $50 \%\left(\mathrm{SOC}_{1000}\right)$, the $\mathrm{SOC}$ value ranges from the minimum of $40 \%\left(\mathrm{SOC}_{1}\right)$ to a maximum of $60 \%\left(\mathrm{SOC}_{2000}\right)$, and the increment $d(\mathrm{SOC})=0.01 \%$, so the error can be neglected.

\section{Calculation and Constraints}

The calculation process of DP is shown in Figure 4.1. The DP control objective is to determine the minimum cost described by the cost-to-go matrix $C$, which is the cumulative fuel consumption $(\mathrm{g} / \mathrm{s})$ over a whole drive cycle. The principle is to find the optimal notes (control/state variables) trajectory in the cost-to-go matrix $C$, where the cost considered in our DP problem is the minimum fuel accumulated consumption calculated by Equation 4.6: 


$$
\begin{aligned}
& J\left(k, \operatorname{SOC}_{.}(k)\right)=\min \left(J\left(k-1, \operatorname{SOC}_{.}(k-1)\right)\right. \\
& \left.+j_{\left(T_{i c e .}(k-1), S_{O C C}(k-1)\right) \rightarrow\left(T_{i c e .}(k), S O C .(k)\right)}\right) \quad \text { Equation } 4.6 \\
& k=1, \cdots, N
\end{aligned}
$$

At each time step $k$ the variables with the following inequality constraints from Equation 4.7 to Equation 4.10 are applied:

$$
\begin{aligned}
\omega_{\text {ice min }} & \leq \omega_{\text {ice }}(k) \leq \omega_{\text {ice max }} & & \text { Equation 4.7 } \\
T_{\text {ice min }}\left(\omega_{\text {ice }}(k)\right) & \leq T_{\text {ice }}(k) \leq T_{\text {ice max }}\left(\omega_{\text {ice }}(k)\right) & & \text { Equation 4.8 } \\
T_{\text {em min }}\left(\omega_{\text {em }}(k), S O C(k)\right) & \leq T_{\text {em }}(k) \leq T_{\text {em max }}\left(\omega_{\text {em }}(k), \operatorname{SOC}(k)\right) & & \text { Equation 4.9 } \\
S O C_{\text {min }} \leq S O C(k) & \leq S O C_{\text {max }} & & \text { Equation 4.10 }
\end{aligned}
$$

Where: $j_{\left(T_{i c e}(k-1), S_{\text {SOC. }}(k-1)\right) \rightarrow\left(T_{i c e}(k), S O C .(k)\right)}$ is one of all the instantaneous minimum fuel consumption from time step $k-1$ to $k$, which depends on the state and control variables $\left(\operatorname{SOC}(\cdot), T_{i c e}(\cdot)\right)$.

$J(k-1, S O C .(k-1))$ is the minimum accumulated cost from the initial time step $k-2$ to $k-1$ at state variable $\operatorname{SOC}(\cdot)$. Figure 4.1 shows the calculation process of DP, where the columns and rows of cost-to-go matrix $C$ correspond to the time grid $N$ and state grid $L$, respectively. Each node of the matrix $C$ can be found between the maximum and minimum SOC from the driving cycle time step $\mathrm{k}_{0}$ to $k_{f}$, which corresponds to the minimum accumulated fuel consumption of the powertrain.

At time step $k$, there are several nodes between the minimum and maximum constraints of the state variable. The minimum cost $J(k, S O C .(k))$ is calculated from the last time step $k-1$ to each of the possible states $S O C .(k)$ at time step $k$ between the limitations of the state variables. The instantaneous minimum fuel consumption $j_{\left(T_{i c e}(k-1), S O C .(k-1)\right) \rightarrow\left(T_{i c e}(k), S O C_{.}(k)\right)}$ between these two points has to be determined. The obtained instantaneous minimum fuel consumption is then added to the cost coming from 
the nodes at time step $k-1$ and saved into the cost-to-go matrix $C$ at the time step $k$ if its cost is less than the existing cost value of the corresponding node.

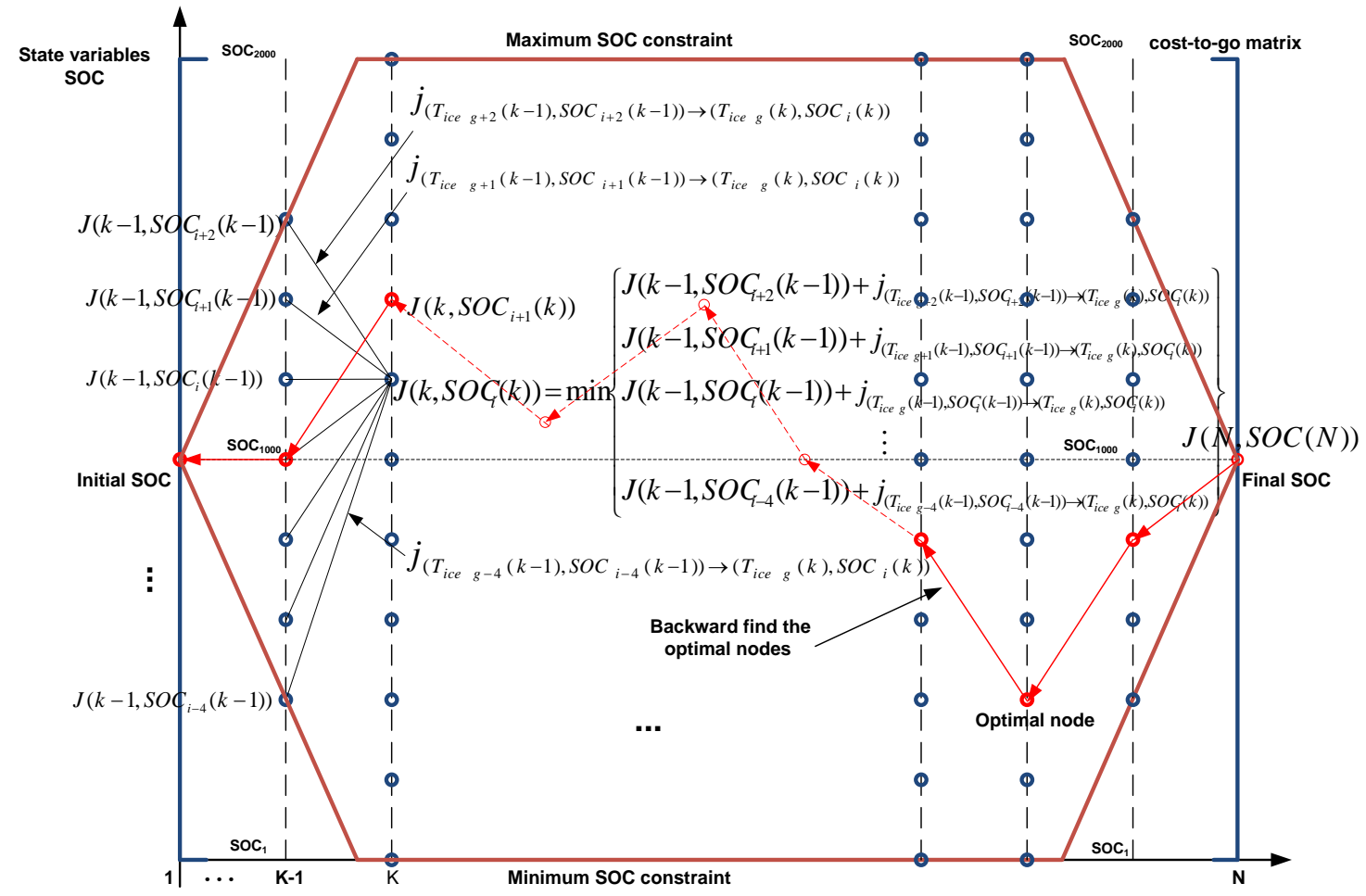

Figure 4.1 Calculation process of DP applied to HEV

The same repeated calculation procedure continues to the next time step $k+1, k+2, \ldots$ until the end time step $N$ of the scheduled route. The final $\operatorname{cost} J(N, \operatorname{SOC}(N))$ is the resulting minimum cumulative fuel consumption of the powertrain during the studied route. After establishing the cost-to-go matrix $C$, the optimal nodes which result in the minimum fuel consumption should be identified. The DP algorithm solution procedure starts at the final desired state value (final SOC) and moves backward state by state each time finding the optimal node for that state until the initial state value (initial SOC). The optimal notes trajectory (red line in Figure 4.1) is identified, and the associated control and state variables are acquired from the identified notes. 
At last, the DP algorithm stores the sequence of optimal powertrain operation (control and state variables) and applies them to control the powertrain during the scheduled trip with the minimum FC.

\subsubsection{DP Simulation Result}

Although the DP and rule-based algorithms are significantly different, the transmission control strategy can be assumed as the same. Accordingly, the gear ratio can be extracted from the rule-based controller (Autonomie software) and used as a lookup table in the DP algorithm. The extracted gear number of the Beijing cycle is shown in Figure 3.7.

Table 4.1 Variables and grids of DP algorithm

\begin{tabular}{|c|c|c|}
\hline & Variables & Grid \\
\hline Stage & Time $[\mathrm{s}]$ & $\mathrm{N} \in[0: 1:$ End $]$ \\
\hline Control & Engine torque $[\mathrm{Nm}]$ & $\mathrm{M} \in\left[-100: 1: T_{\text {ice_max }}\right]$ \\
\hline State & SOC $[-]$ & $\mathrm{L} \in[0.4: 0.0001: 0.6]$ \\
\hline
\end{tabular}

The validated hybrid bus model was utilized to simulate the operation of a hybrid transit bus. The initial and final SOC are set to 0.5 , the quantization increment of the SOC, ICE torque $\mathrm{T}_{\text {ice, }}$ and time step $k$ is set to $0.01 \%, 1 \mathrm{Nm}$, and $1 \mathrm{~s}$, respectively. The engine brake torque is $-100 \mathrm{Nm}$ as shown in Table 4.1 .

In this section, the results of the DP procedure are compared to those of the rule-based algorithm applied to Beijing cycles. The potential of DP in improving the FE is also examined using a revised WVU-CSI cycle to cover more versatile driving conditions. The revised WVU-CSI cycle combined the WVU City cycle, WVU Suburban cycle, and WVU Interstate cycle. The speed of WVU Interstate cycle is scaled by a factor of 0.7 due to power limitations of the hybrid bus as shown in Figure A. 1.

The comparison of simulation results, the distribution of power source and power operation points are shown in Figure 4.2-4.4 and Figure A. 2-A. 4 for the Beijing cycle and WVUCSI cycle, respectively. 


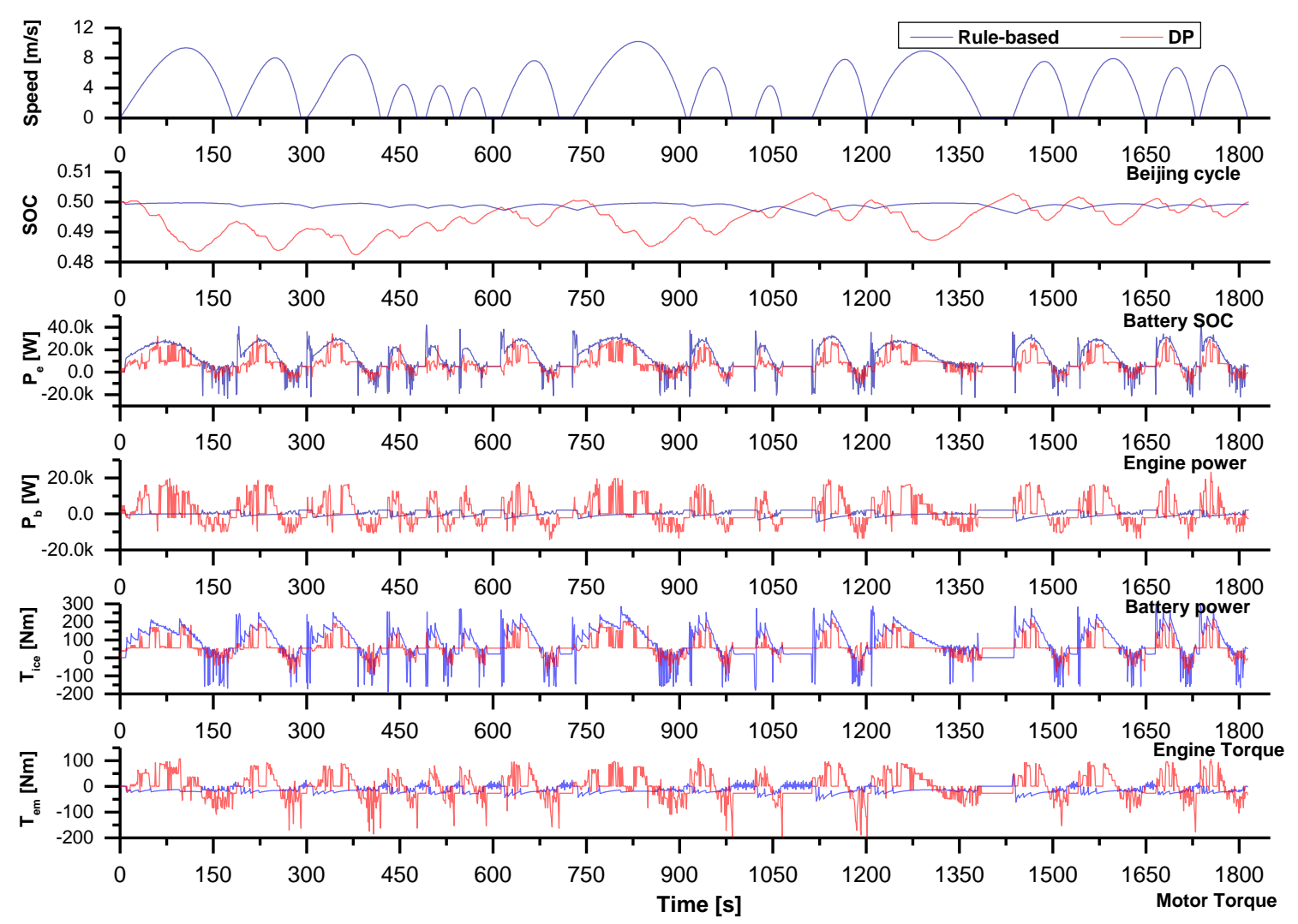

Figure 4.2 Comparison of trajectories between rule-based and DP over the Beijing cycle

As shown in Figure 4.2 and Figure A. 2, although the SOC starts and finishes at the same initial and final SOC 0.5, the fluctuation of SOC trajectories derived using DP is bigger than that derived using rule-based algorithm. The DP algorithm consumes more electrical energy to propel the vehicle or assist the engine than rule-based controller. The increased electric only operation time caused FE improvement. In the study of the WVU-CSI cycle, the SOC trajectory shows that DP charges the battery above the reference SOC in lower power requested driving conditions (WVU-City cycle and WVU-Suburban cycle) then uses the battery power in higher power demand situations (WVU-Interstate cycle). This is because that DP has prior knowledge of the entire driving cycle and the DP model assumes that all kinetic energy is either consumed due to the power loss or recovered by the brake energy recovery system without involving mechanical brake. 

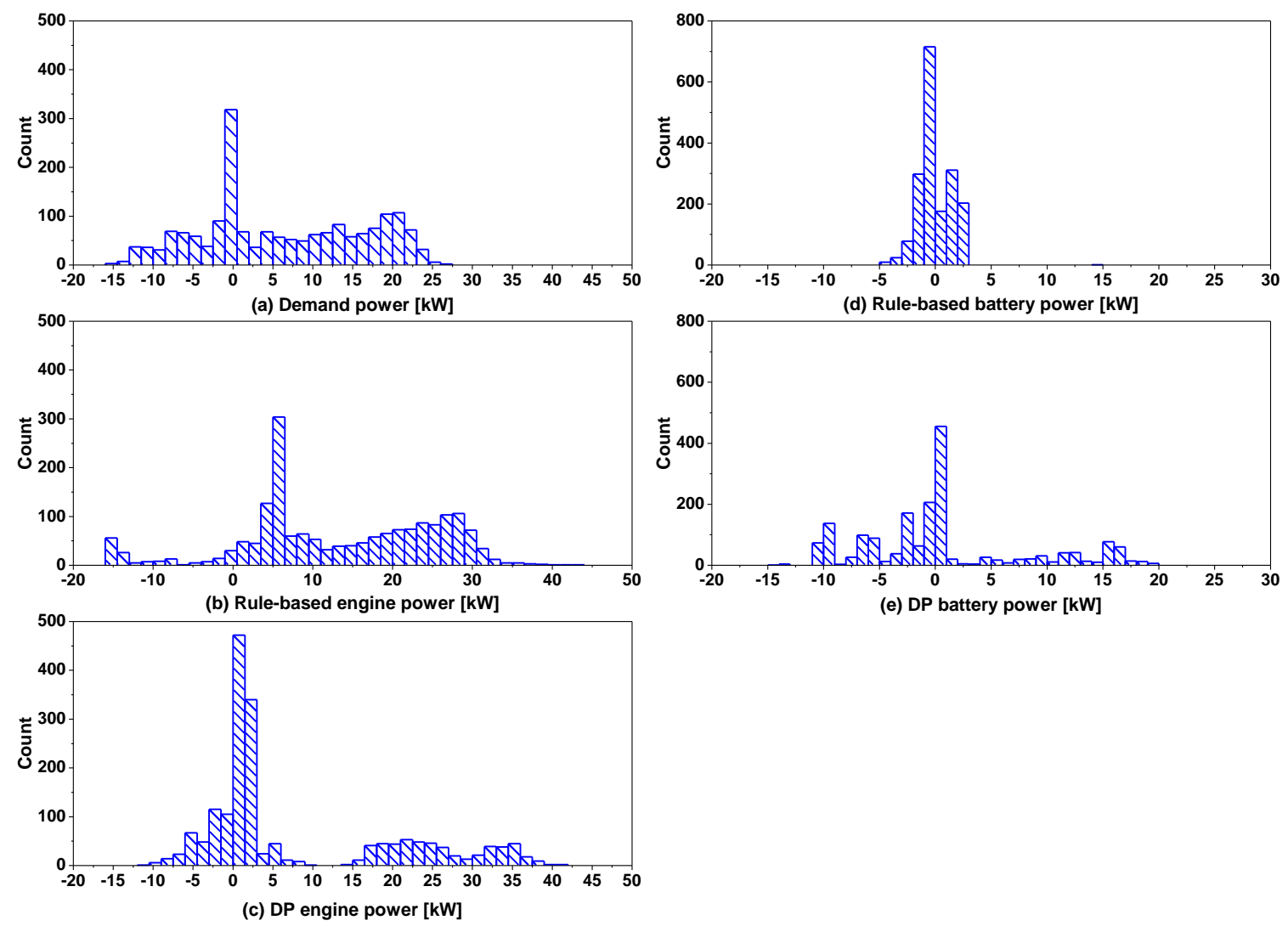

(e) DP battery power [kW]

Figure 4.3 Distribution of the (a) demand power, (b) rule-based engine power, (c) DP engine power, (d) rule-based battery power and (e) DP battery power over the Beijing cycle.

The distribution of the demand power, engine power and battery power are shown in Figure 4.3 and Figure A. 4. In the study of the Beijing cycle (Figure 4.3), the DP battery power range $(-15 \mathrm{~kW}$ to $20 \mathrm{~kW})$ is greater than that of rule-based controller $(-5 \mathrm{~kW}$ to $3 \mathrm{~kW})$ since the power to propel the vehicle was mostly provided by the engine in the rule-based controller. On the contrary, the engine power range and frequency optimized using DP is smaller than that of rule-based controller. There is no power distribution between $8 \mathrm{~kW}$ to13kW as shown in Figure 4.3-c.

In the similar results are shown in the study of the WVU-CSI cycle (Figure A. 4), the DP battery power range $(-30 \mathrm{~kW}$ to $80 \mathrm{~kW})$ is greater than that of the rule-based controller ($35 \mathrm{~kW}$ to $23 \mathrm{~kW}$ ) while the DP engine power range is smaller than that of rule-based 
controller. The simulation results indicate that the control strategies developed using DP clearly favors more battery power usage.

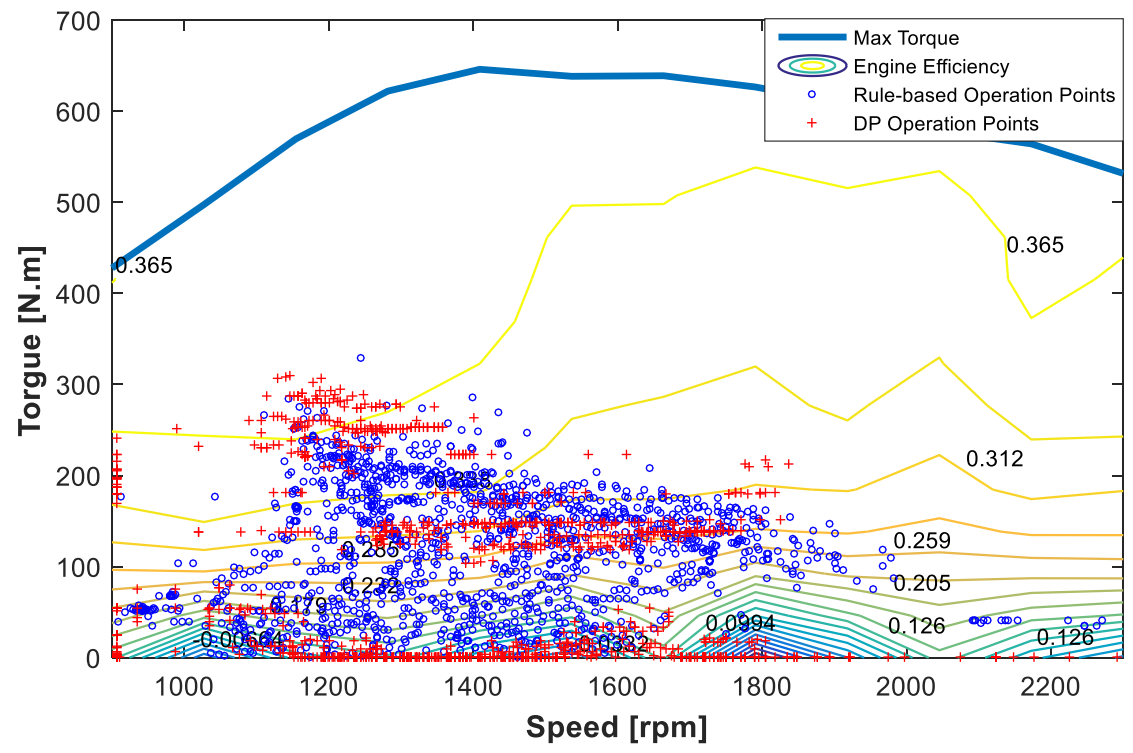

(a) Engine speed, torque, and efficiency

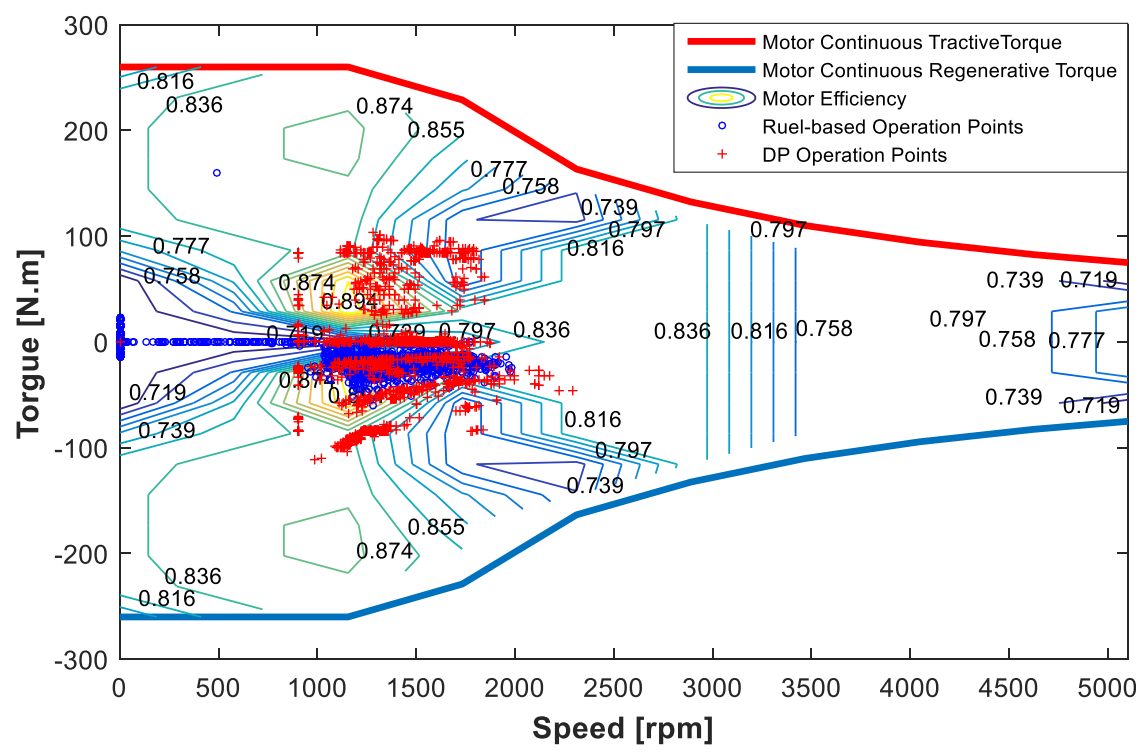

(b) Motor speed, torque and efficiency

\section{Figure 4.4 Rule-based and DP power source operation points distribution maps over the Beijing cycle}

This phenomenon can be further validated by comparing the power operating points of the engine and motor as shown in Figure 4.4 and Figure A. 3. The DP operating points on the 
engine efficiency map are almost in the high torque optimum region to charge the battery or the idle torque region in the low power request driving condition, which will turn off the engine and reduce the $\mathrm{FC}$ and $\mathrm{NO}_{\mathrm{x}}$ emissions. As shown in Figure 4.4-a, the rule-based operating points are farther from the high efficiency zone. The engine was operated over a wider area especially in the low efficiency region.

Table 4.2 compares the $\mathrm{FE}$ and $\mathrm{NO}_{\mathrm{x}}$ emissions simulated using DP with those achieved by rule-based control strategies over the Beijing cycle. The application of DP was found to reduce the $\mathrm{FC}$ and $\mathrm{NO}_{\mathrm{x}}$ emissions by $58.44 \%$ and $21.12 \%$, respectively.

Table A. 1 compares the FE and $\mathrm{NO}_{\mathrm{x}}$ emissions simulated using DP with those achieved by rule-based control strategies over the WVU-CSI cycle. The application of DP was found to reduce the $\mathrm{FC}$ and $\mathrm{NO}_{\mathrm{x}}$ emissions by $34.02 \%$ and $17.38 \%$, respectively.

Table 4.2 DP and rule-based simulation results for Beijing cycle

\begin{tabular}{|c|c|c|c|}
\hline & FE $(\mathbf{m p g})$ & FC $($ gram/mi) & NOx $(\mathbf{g r a m} / \mathbf{m i})$ \\
\hline Rule-based & 7.05 & 448.64 & 22.40 \\
\hline DP & 11.17 & 281.81 & 17.67 \\
\hline
\end{tabular}

\subsubsection{The Potential of DP in Improving Cost Function}

As shown in Figure 4.2, the engine and motor were turned on and off more frequently in DP simulation results. To avoid the excessive number of these unfavorable events and make the model feasible, a penalty in terms of engine on/off and motor current was added. The DP with an improved cost function can be expressed as Equation 4.11:

$$
J=\sum_{k=0}^{N-1}\left(\text { fuel }_{k}+\alpha\left|I_{k}\right|+\beta \Delta E_{\text {on } / o f f, k}\right), \mathrm{k}=1,2 \cdots \mathrm{N} \quad \text { Equation } 4.11
$$

Where $I$ is the battery current, $\Delta E_{\text {on } / \text { off }}=\mid E_{k}($ on, off $)-E_{k-1}($ on, off $) \mid$ is the absolute value of the difference between engine on or off status, $\alpha$ and $\beta$ are the weighting factors, which have to be adjusted to improve drivability of the simulated solution. After testing, it was found that $\alpha=0.02$ and $\beta=5$ can provide better simulation results. The engine power 
and motor power trajectories shown in Figure 4.5 represent the optimal operation between the two power sources in achieving the best fuel economy.

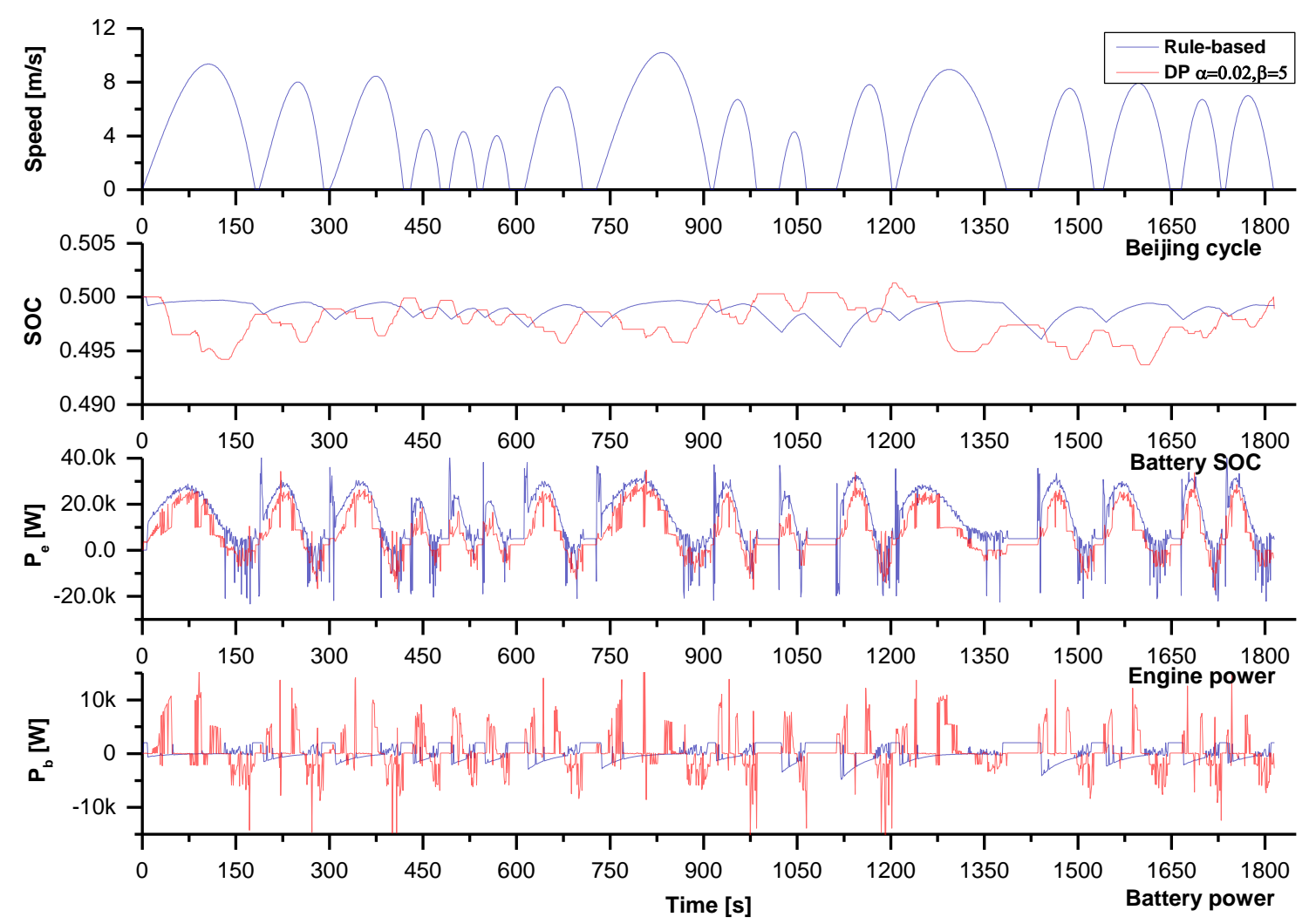

Figure 4.5 The simulated results of the rule-based controller and DP with an improved cost function over the Beijing cycle

\subsubsection{The Trade-off between FE and $\mathrm{NO}_{\mathrm{x}}$ Emissions}

In general, the operation of an engine at high load and high speed usually leads to a higher average operating temperature and higher $\mathrm{NO}_{\mathrm{x}}$ emissions. For example, Rousseau \& et al. (2002) investigated certain trade-offs of a vehicle. An increase of FC by $13 \%$ was accompanied with a $40 \%$ reduction in $\mathrm{NO}_{\mathrm{x}}$ emissions.

In this research, the trade-off between $\mathrm{NO}_{\mathrm{x}}$ emissions and $\mathrm{FE}$ is examined by adding a weighting factor $\gamma$, and the DP cost function is revised as Equation 4.12:

$$
\begin{gathered}
J=\sum_{\mathrm{k}=0}^{\mathrm{N}-1}\left(\text { fuel }_{k}+\alpha\left|I_{k}\right|+\beta \Delta \mathrm{E}_{\mathrm{on} / o f f, \mathrm{k}}+\gamma N O_{X_{k}}\right) \\
\mathrm{k}=1,2 \cdots \mathrm{N}
\end{gathered}
$$


In the Beijing cycle, the trade-offs between $\mathrm{FE}$ and $\mathrm{NO}_{\mathrm{x}}$ emissions are studied by varying the weighting factor over a range of $\gamma \ni[0,5,10,15,20,25,30]$. Figure 4.6 shows the variation of the FE penalty with changes in $\mathrm{NO}_{\mathrm{x}}$ emissions simulated using DP with different $\gamma$ values. The reduction in $\mathrm{NO}_{\mathrm{x}}$ emissions is always accompanied with significant deterioration in $\mathrm{FE}$. Increasing $\gamma$ leads to significant $\mathrm{NO}_{\mathrm{x}}$ reduction while causing a small FE increase. The penalty to fuel economy become significant when $\gamma$ is over 15 . The case with $\gamma=15$ seem to achieve the best trade-off, a $13.5 \%$ reduction in $\mathrm{NO}_{\mathrm{x}}$ emissions is achieved with a $2.7 \%$ increase in FC when compared to optimization based on the fuel consumption only case $(\alpha=0, \beta=0, \gamma=0)$.

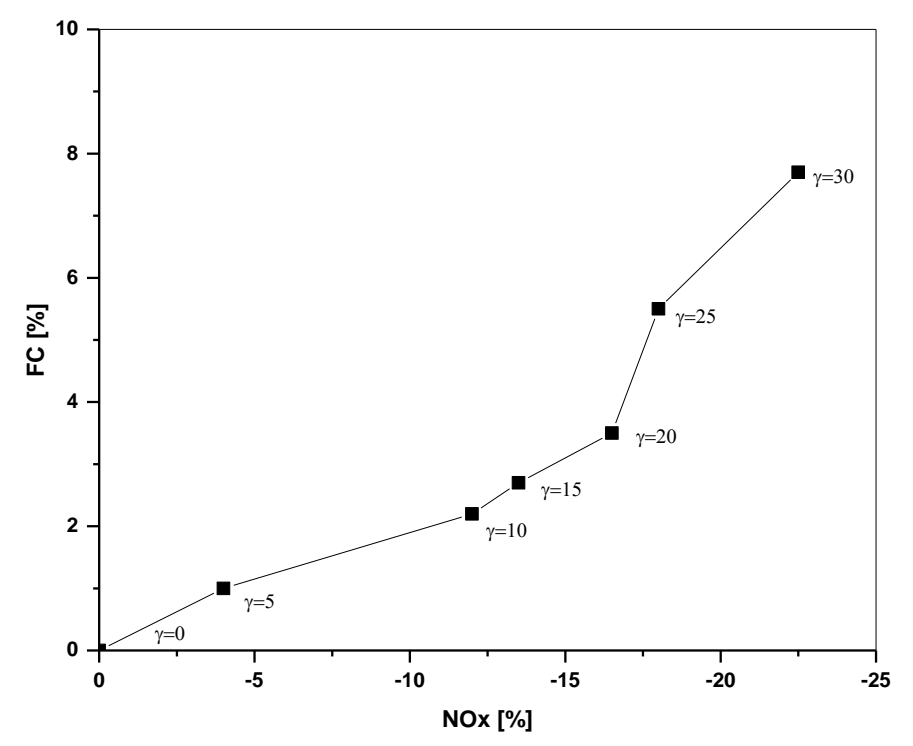

Figure 4.6 The trade-off between FE and $\mathrm{NO}_{\mathrm{x}}$ emissions simulated using DP

Figure 4.7 shows the simulated impact of a fuel penalty on the operation of a hybrid vehicle over the Beijing cycle. In the case simulated with $\alpha=0.02, \beta=5$, the on/off frequency of the engine and motor was reduced significantly and against the unexpected fuel consumption from the low efficiency operation zone. However, the $\mathrm{NO}_{\mathrm{x}}$ emissions was increased due to the operation of the engine over a high load. The trade-off between the lower on/off 
frequency and the enhanced power from engine still reduced the $\mathrm{NO}_{\mathrm{x}}$ emissions from $17.67 \mathrm{~g} / \mathrm{mile}(\alpha=0, \beta=0, \gamma=0)$ to $17.03 \mathrm{~g} / \mathrm{mile}(\alpha=0.02, \beta=5, \gamma=0)$.

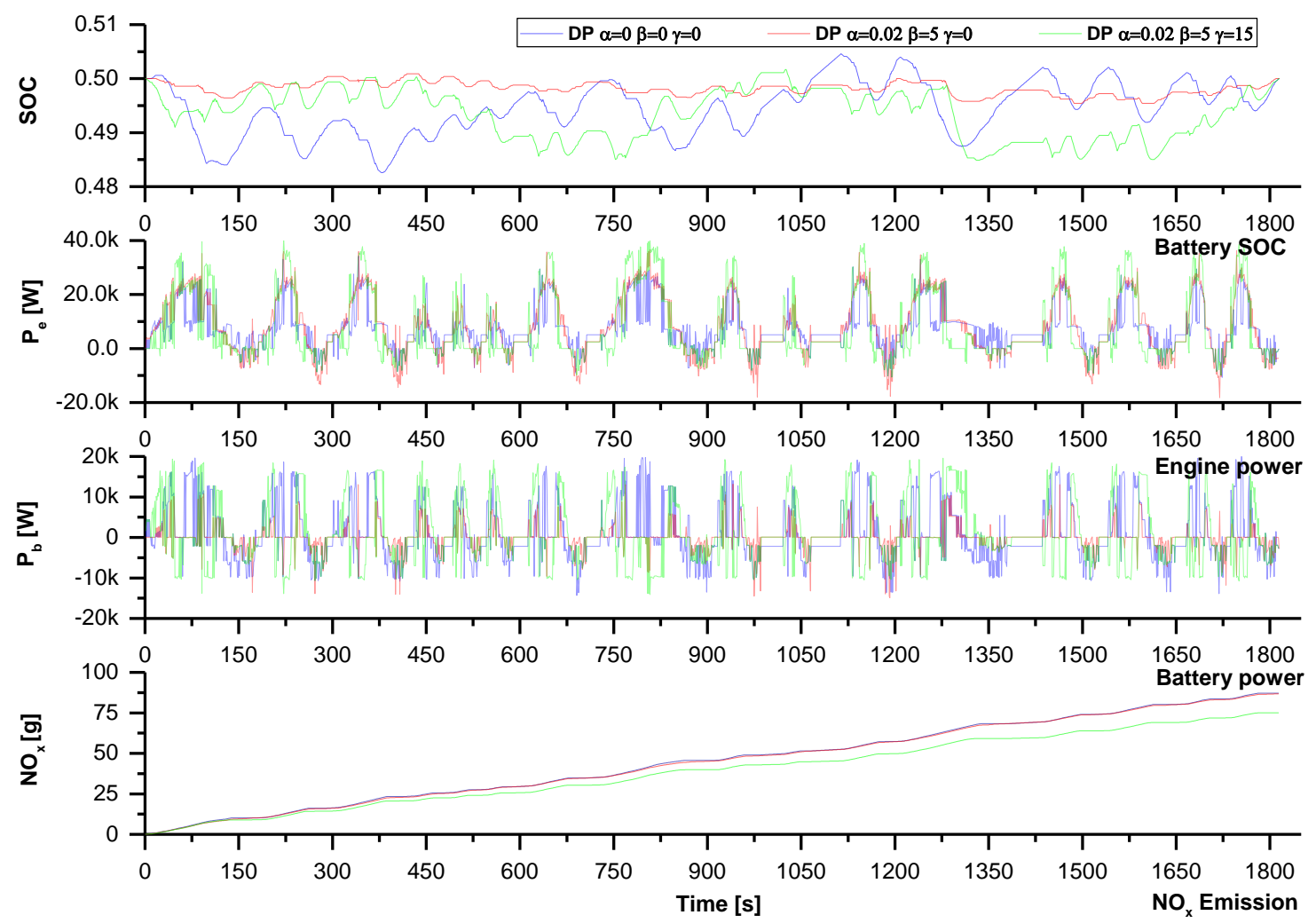

Figure 4.7 The simulated $\mathrm{NO}_{\mathrm{x}}$ emissions with or without an improved cost function over the Beijing cycle simulated using DP

The $\mathrm{NO}_{\mathrm{x}}$ trajectories of the case simulated with $\alpha=0.02, \beta=5$, and $\gamma=15$ show that this case charges/discharges the battery more than the other two cases, and has an added the benefit of more battery power utilized, which improves the $\mathrm{FE}$ and decreases the $\mathrm{NO}_{\mathrm{x}}$ emissions. Table 4.3 compares the FE, FC, and $\mathrm{NO}_{\mathrm{x}}$ emissions simulated using DP and the rule-based control strategy. The case simulated with $\alpha=0.02, \beta=5$, and $\gamma=0$ was able to further improve the fuel economy and decrease $\mathrm{NO}_{\mathrm{x}}$ emissions, indicating the benefit achieved by the proposed less frequent on/off operation of the engine and motor. The proposed tradeoff case $(\alpha=0.02, \beta=5, \gamma=15)$ was able to further reduce $\mathrm{NO}_{\mathrm{x}}$ emissions by $10.3 \%$ with a $4.3 \%$ penalty in fuel economy compared to the no tradeoff case $(\alpha=0.02, \beta=5, \gamma=0)$. 
Table 4.3 DP with or without penalty and rule-based simulation results for the Beijing cycle

\begin{tabular}{|c|c|c|c|}
\hline & FE $(\mathbf{m p g})$ & FC $(\mathbf{g r a m} / \mathbf{m i})$ & NOx $(\mathbf{g r a m} / \mathbf{m i})$ \\
\hline Rule-based & 7.05 & 448.64 & 22.40 \\
\hline DP $(\boldsymbol{\alpha}=\mathbf{0}, \boldsymbol{\beta}=\mathbf{0}, \boldsymbol{\gamma}=\mathbf{0})$ & 11.17 & 281.81 & 17.67 \\
\hline DP $(\boldsymbol{\alpha}=\mathbf{0 . 0 2}, \boldsymbol{\beta}=\mathbf{5}, \boldsymbol{\gamma}=\mathbf{0})$ & 11.35 & 274.23 & 17.03 \\
\hline DP $(\boldsymbol{\alpha}=\mathbf{0 . 0 2}, \boldsymbol{\beta}=\mathbf{5}, \boldsymbol{\gamma}=\mathbf{1 5})$ & 10.86 & 290.09 & 15.28 \\
\hline
\end{tabular}

Note: $\alpha, \beta, \gamma$ is the weighting factors of battery current, engine on/off, and $\mathrm{NO}_{\mathrm{x}}$ emissions

\subsection{Equivalent Consumption Minimization Strategy}

ECMS is a power distribution strategy based on PMP, which is defined as finding the local optimal solution for the power proportion of electric and ICE at each time step under consideration of an energetic equivalence factor (Kutter and Bäker 2010). The ECMS is developed by calculating the sum of fuel consumption as the FC by the ICE and equivalent FC of the electrical system (Delprat, Guerra and Rimaux 2002, Paganelli, et al. 2002).

\subsubsection{Pontryagin's Minimum Principle}

The PMP is used in optimal control theory to find the best possible control for taking a dynamic system from one state to another, especially in the presence of constraints for the state $x$ or input $u$ controls.

Minimize the cost function, which can be described by:

$$
J=\Psi(x(T))+\int_{0}^{T} L(x(t), u(t)) d t
$$

Where $T$ is the terminal time of the system. The constraints on the system dynamics can be adjoined to the Lagrangian $L$ by introducing a time varying Lagrange multiplier vector $\lambda$, whose elements are noted as the co-states of the system. This motivates the construction of the Hamiltonian $H$ (Kirk 2012).

$$
\begin{gathered}
H(\lambda(t), x(t), u(t), t)=\lambda(t) f(x(t), u(t))+L(x(t), u(t)) \\
t \in[0, T]
\end{gathered}
$$

Equation 4.14

Necessary Conditions from PMP: 
I. State and co-state equation:

$$
\begin{array}{cc}
\dot{x}(t)=f(x(t), u(t)) & \text { Equation } 4.15 \\
\dot{\lambda}(x)=-\frac{\partial H}{\partial x}(\lambda(t), x(t), u(t), t) & \text { Equation } 4.16
\end{array}
$$

II. Initial condition and terminal conditions when the final state $x(T)$ and final time $T$ are free (i.e., its differential variation is not zero):

$$
\begin{array}{cc}
x(0)=x_{0} & \text { Equation } 4.17 \\
\lambda(T)=\Psi_{x}(x(T)) & \text { Equation } 4.18 \\
\Psi_{T}(x(T))+H(T)=0 & \text { Equation } 4.19
\end{array}
$$

III. Minimum condition: for all $t \in[0, T]$ and for all permissible control input $u$

$$
H(\lambda(t), x(t), u(t), t) \leq H(\lambda(t), x(t), u, t) \quad \text { Equation } 4.20
$$

PMP needs to satisfy the conditions in Equation 4.15 to Equation 4.20 to solve an optimal control problem.

\subsubsection{Application of ECMS in Developing Hybrid Vehicle Control Strategies}

The ECMS was derived from PMP and converted the uncertainty conditions to the correct optimal value of the co-state, which was a time-variable equivalent factor (Paganelli, et al. 2002). In the hybrid vehicle model, $\operatorname{SOC}(t)$ is considered as the state $x(t)$, engine torque $T_{i c e}(t)$ as the input control $u(t)$, and the fuel consumption rate $m_{f}$ as $L$ function which is independent of $\operatorname{SOC}(t)$. From the Hamiltonian function Equation 4.14 and state Equation 4.15, the Hamiltonian of HEV can be expressed as:

$$
\begin{gathered}
H\left(\lambda(t), \operatorname{SOC}(t), T_{i c e}(t), t\right)=m_{f}\left(T_{i c e}(t), t\right) \\
+\lambda(t) \operatorname{SOC}\left(\operatorname{SOC}(t), T_{i c e}(t), t\right)
\end{gathered}
$$

Equation 4.21

Where the co-state $\lambda(t)$ is sometimes referred to as the Lagrange multiplier or adjoint state and taken as the $\lambda(t)$ that converts battery energy consumption to equivalent fuel consumption. 
According to the optimal control policy of PMP, Equation 4.21 must satisfy the necessary conditions for optimality listed below.

The state equation and the co-state equation can be expressed as Equation 4.22 and Equation 4.23, respectively.

$$
\begin{aligned}
& \operatorname{SOC}(t)=\frac{\partial H}{\partial \lambda(t)}=f\left(\operatorname{SOC}(t), T_{i c e}(t)\right) \\
& \dot{\lambda}(t)=-\lambda(t) \frac{\partial \operatorname{SOC}\left(\operatorname{SOC}(t), T_{i c e}(t), t\right)}{\partial \operatorname{SOC}(t)}
\end{aligned}
$$

The SOC boundary condition was set as Equation 4.24 to ensure battery charge sustaining for the optimal control of parallel HEVs.

$$
\operatorname{SOC}(0)=\operatorname{SOC}(T)=S O C_{\text {initial }}
$$

Equation 4.24

For optimality, the minimum condition in Equation 4.20 should be considered to determine the optimal control variable, $T_{i c e}(t)$ at every time step.

Therefore, the Hamiltonian aimed at instantaneous minimization has the physical meaning of an instantaneous equivalent fuel consumption function described by

$$
m_{f, e q u}(t, u)=m_{f}(t, u)+\lambda(t) \cdot \frac{P_{e l e}(t, u)}{H_{f}}
$$

Equation 4.25

where $m_{f, e q u}(t, u)$ is the instantaneous equivalent fuel consumption, $m_{f}(t, u)$ is instantaneous fuel consumption of the ICE, $P_{e l e}(t, u)$ is the instantaneous electrochemical power stored in battery, $\lambda(t)$ is the equivalent factor that converts electrical system power into the equivalent $\mathrm{FC}$, and $H_{f}$ is the lower heating value of the fuel.

In the original ECMS formulation, the $\lambda(t)$ represents the chain of efficiencies through which the chemical energy in the fuel is transformed into electrical power and vice-versa, based on each operating condition of the powertrain and driving mission (Onori and Serrao 2011). Accounting for the loss of transforming the battery energy into fuel and vice versa, 
the losses involved are lumped into the $\lambda(t)$. The equivalent factor $\lambda(t)$ is a time-variant value based on the change of battery SOC and vehicle operating conditions.

In a charge-sustaining situation, the SOC mainly operates in a narrower range, e.g., from 0.5 to 0.7 ; hence, the voltage and the resistance may not vary so much in the range. In this case, from Equation 4.23

$$
\dot{\lambda}(t)=-\lambda(t) \frac{\partial S \dot{O} C\left(\operatorname{SOC}(t), T_{i c e}(t), t\right)}{\partial S O C(t)} \approx 0 \Rightarrow \lambda(t)=\lambda \quad \text { Equation } 4.26
$$

When ignoring the change of battery SOC, a time-invariant value $\lambda$ of the certain driving condition exists. $\lambda$ will be the optimal equivalent factor of the ECMS over the fixed driving condition.

Accordingly, $\lambda$ will be the time variant value when the impact of SOC on the equivalent factor is accounted for. If the change of SOC is ignored, $\lambda$ will be the time-invariant value as shown in the Equation 4.27.

$$
\begin{gathered}
E F=\lambda(t) \text { Consider the change of SOC } \\
E F=\lambda \quad \text { Ignore the change of SOC }
\end{gathered}
$$

Equation 4.27

If the transit bus route is unknown, it is challenging to determine the suitable time-invariant $\lambda$, which means that the constant $\lambda$ need to be estimated. Also, $\lambda$ is considered as an optimization variable that acts as a single tuning parameter, which corresponds to the "equivalent fuel" price of electrical energy. The larger the value of $\lambda$, the more expensive electrical energy is, and the more attractive it is to charge the battery through engine and brake energy recovery. The smaller the value of $\lambda$, the cheaper electrical energy is, and the more beneficial it is if electrical power is used to propel the vehicle conjointly with engine power. 


\subsubsection{ECMS Simulation Result}

In order to compare the performance of the ECMS with the global optimal solution obtained from DP, the pre-transmission parallel HEV (Figure 3.1) is modeled in the MATLAB/ m script environment. The characteristics of the vehicle used here are shown in Table 3.1. Based on the assumption, the gear ratio of the ECMS can be extracted from Autonomie and formatted as a lookup table in the model, and the initial and final SOC are set to 0.5 . The minimum and maximum SOC value is 0.4 and 0.6 , respectively. The ECMS model primarily uses simplified quasi-static map based models for all the ICE and EM as shown in Figure 3.2, Figure 3.3 and Figure 3.4. At each time step the variables should satisfy the inequality constraints, as shown in Equation 4.7 to Equation 4.10.

The ECMS algorithm is very sensitive to the variation of $\lambda$, which results in significant fluctuation of the SOC trajectory. To keep the SOC of the ESS within a specified limit $\left(S O C_{\min }<\forall S O C<S O C_{\max }\right)$ during the driving period, the value of $\lambda$ must be modified when the SOC trajectory is close to the maximum or minimum values, through the equation expressed as,

$$
\begin{array}{ll}
\lambda(t)=\lambda_{\text {max }} & \forall S O C<S O C_{\text {min }} \\
\lambda(t)=\lambda_{\text {min }} & \forall S O C>S O C_{\text {max }}
\end{array}
$$

In this section, the simulation results of the ECMS procedures are compared to those of DP over the Beijing cycle. In order to validate the performance of the optimal ECMS for different driving conditions, the WVU-CSI cycle was chosen as the testing cycle, and the simulation results are shown in the Appendix B.

Figure 4.8 (Beijing cycle) and Figure B. 1 (WVU-CSI cycle) compare the SOC, engine power, battery power and FC calculated using an optimal ECMS and DP, respectively. The SOC, $P_{e}, P_{b}$ and FC trajectories are similar and close between DP and ECMS with $\lambda_{o p t}$, as shown in Figure 4.8 and Figure B. 1. 
This phenomenon can be further validated by comparing the power operating points of the engine and motor as shown in Figure 4.9 and Figure B. 2. The electric motor is operated mainly in the efficient regions similar to the DP solution as shown in Figure 4.9-b and Figure B. 2-b.

The corrected fuel economy $F E_{\text {corr }}$ is defined as the sum of the mass of fuel consumed along with a correction for the net change in battery SOC. The $F E_{c o r r}$ is expressed as

$$
F E_{c o r r}=F E+\frac{\tau \cdot \Delta S O C \cdot Q_{b}}{\eta_{p a t h} \cdot H_{f}}
$$

Where $F E$ is the fuel economy; $Q_{b}$ is the nominal battery capacity; $H_{f}$ is the lower heating value of the fuel; $\eta_{\text {path }}$ is the approximated efficiency of the drivetrain used in regenerating/discharging the battery; $\triangle S O C$ is the change in SOC; $\tau$ is the unit convert parameter.

Table 4.4 shows the simulated fuel economy of the Beijing cycle. The optimal $\lambda$ value $\left(\lambda=\lambda_{\text {opt }}=2.227\right)$ is selected with the corrected fuel economy $F E_{c o r r}=10.81 \mathrm{mpg}$ and $\triangle S O C=-0.04 \%$, which is only a $3.22 \%$ gap to DP simulated fuel economy.

The corrected fuel economy gap to that observed using DP is calculated by Equation 4.30.

$$
\text { Gap to } D P=\frac{F E_{(D P)}-F E_{\operatorname{corr}(\cdot)}}{F E_{(D P)}}
$$

As shown in Table B. 1, the operation of this bus over the WVU-CSI cycle with the optimal ECMS control strategies burn almost $6 \%$ more fuel than the operation of this bus on the control strategy developed using DP.

Table 4.4 DP $(\alpha=\beta=\gamma=0)$ and ECMS with optimal $\lambda$ simulation results over the Beijing cycle

\begin{tabular}{|c|c|c|c|c|}
\hline & FE $(\mathbf{m p g})$ & $\boldsymbol{\Delta S O C}$ & $\boldsymbol{F E}$ corr $(\mathbf{m p g})$ & Gap to DP \\
\hline Optimal ECMS $\left(\boldsymbol{\lambda}=\boldsymbol{\lambda}_{\text {opt }}=\mathbf{2 . 2 2 7}\right)$ & 10.80 & $-0.04 \%$ & 10.81 & $3.22 \%$ \\
\hline DP $(\boldsymbol{\alpha}=\mathbf{0}, \boldsymbol{\beta}=\mathbf{0}, \boldsymbol{\gamma}=\mathbf{0})$ & $\mathbf{1 1 . 1 7}$ & $\mathbf{0 . 0 0}$ & $\mathbf{1 1 . 1 7}$ & $\mathbf{0 . 0 0 \%}$ \\
\hline
\end{tabular}




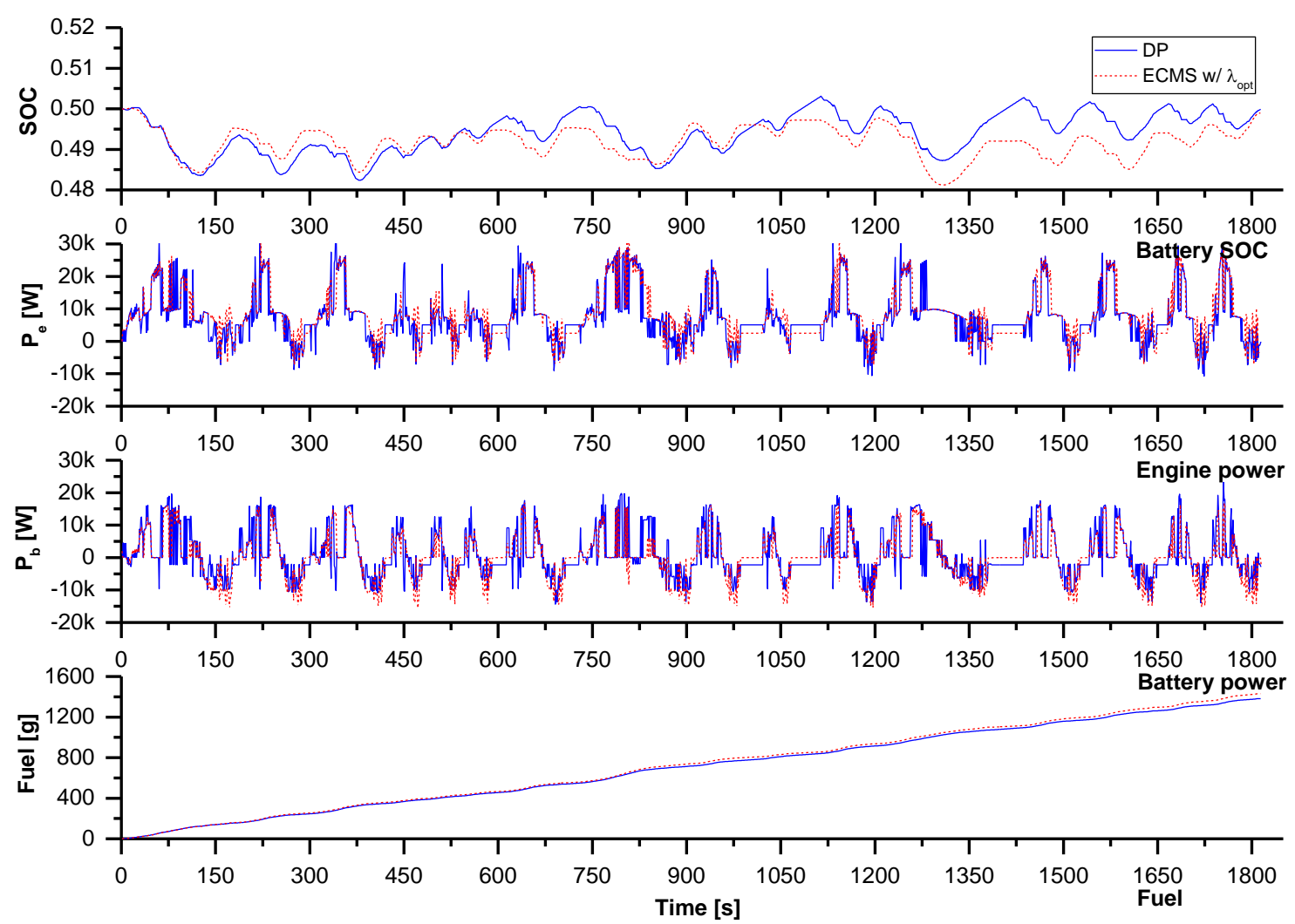

Figure 4.8 The comparison between DP $(\alpha=\beta=\gamma=0)$ and ECMS with optimal $\lambda$ simulation results over the Beijing cycle

Figure 4.9-a and Figure B. 2-a. shows that the ICE worked on the high efficient and torque region of the engine map. It is evident that this bus operated on the DP algorithm charges/discharges the battery more often than the optimal ECMS and achieves the benefit of more battery power utilized. The ECMS model assumes that all kinetic energy is either consumed due to the power loss or recovered by the brake energy recovery system without involving mechanical brake. 


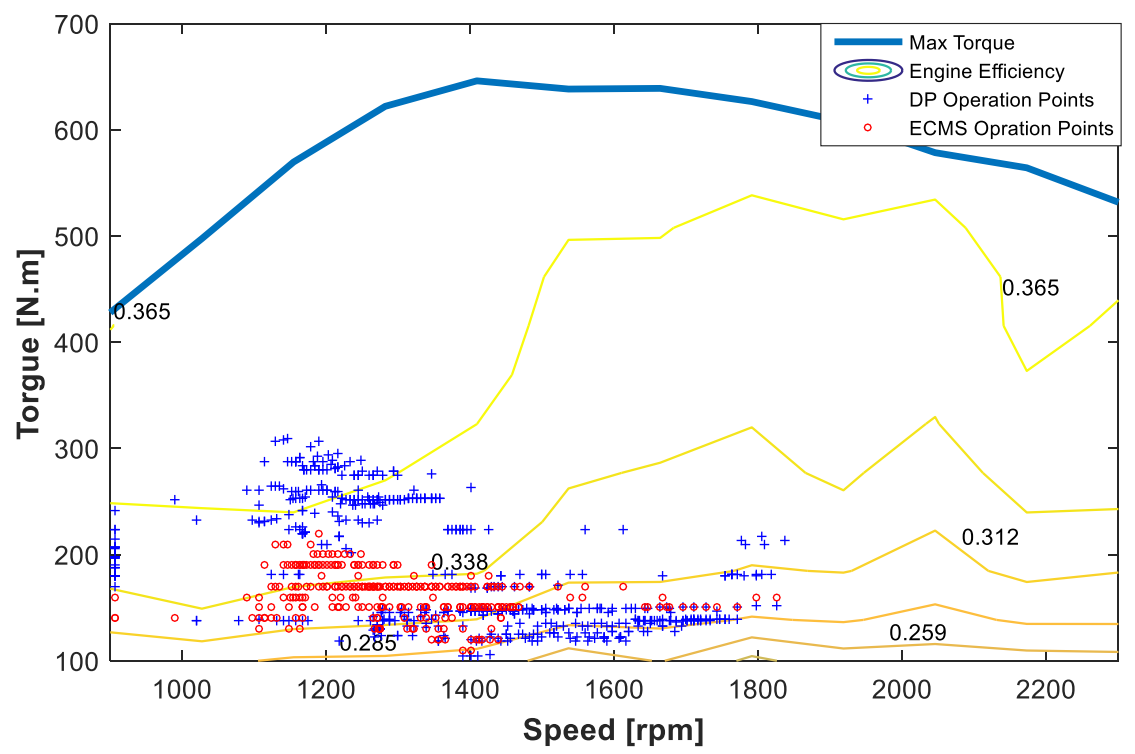

(a) Engine speed, torque and efficiency

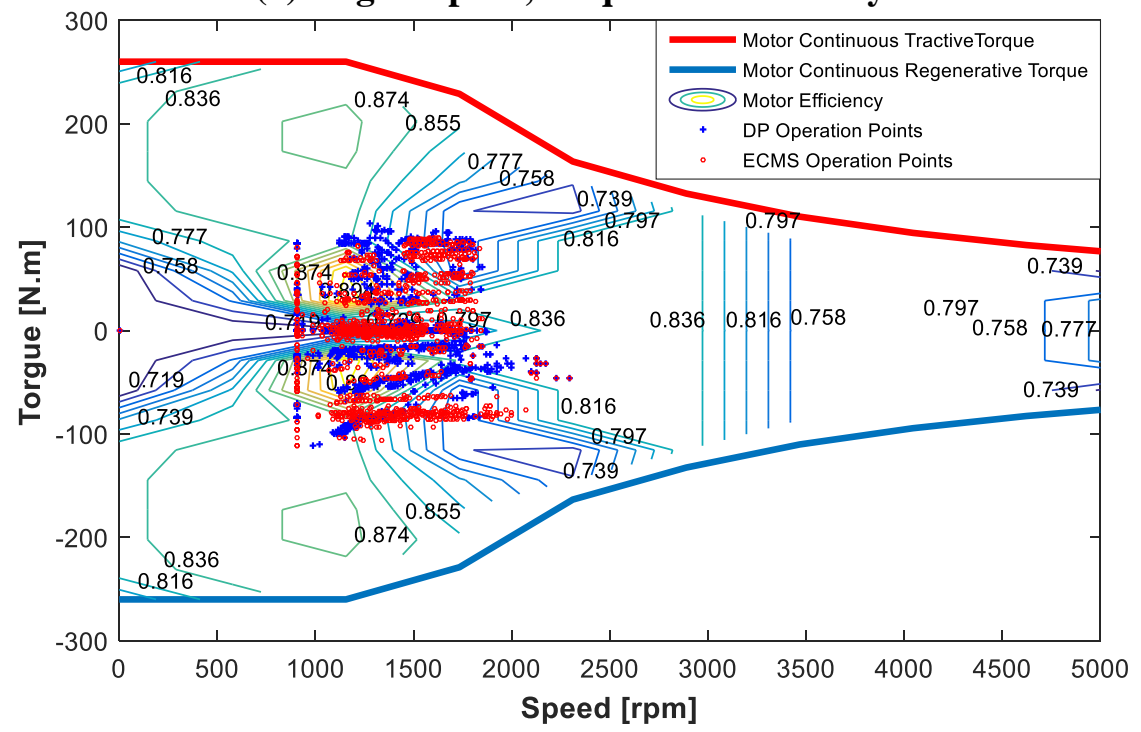

(b) Motor speed, torque and efficiency

Figure 4.9 DP $(\alpha=\beta=\gamma=0)$ and ECMS with optimal $\lambda$ power source operation points distribution in maps (Beijing cycle)

\subsubsection{ECMS with Penalty Function}

Since the transit bus route information is not known in advance, an approximated $\lambda$ has to be selected. In Figure 2.6, the estimated time-invariant $\lambda$ strongly affects the charge and discharge of the battery. A minor increase in $\lambda(2.1$ to 2.3$)$ will result in serious discharging/charging of the battery. 
To ensure a charge-sustainable solution for a parallel hybrid vehicle, the penalty equation was taken into consideration in the ECMS algorithm (De Jager, Steinbuch and Keulen 2008, Onori, Serrao and Rizzoni 2010); that is,

$$
\begin{array}{lll}
P(S O C)=1+\left|\frac{S O C_{\text {ref }}-S O C}{S O C_{\text {ref }}-S O C_{\text {min }}}\right|^{n} & , \forall S O C<S O C_{\text {ref }} & \\
P(S O C)=1 & , \forall S O C=S O C_{\text {ref }} & \text { Equation } 4.31 \\
P(S O C)=1-\left|\frac{S O C_{\text {ref }}-S O C}{S O C_{\text {max }}-S O C_{\text {ref }}}\right|^{n} & , \forall S O C>S O C_{\text {ref }} &
\end{array}
$$

where $S O C_{\min }, S O C_{\max }$ and $S O C_{\text {ref }}$ represent the minimum, maximum and reference SOC value, typically $40 \%, 60 \%$ and $50 \%$, respectively, and $\mathrm{n}$ is the exponential coefficient governing the shape of the correction function to control the reaction rates of the penalty weight. The penalty function corrects $\lambda$ whenever the current SOC deviates from $S O C_{r e f}$. The penalty $P(S O C)$ is inappreciable whenever SOC is close to the reference value and increases nonlinearly as the SOC deviates from the reference as shown in Figure 4.10.

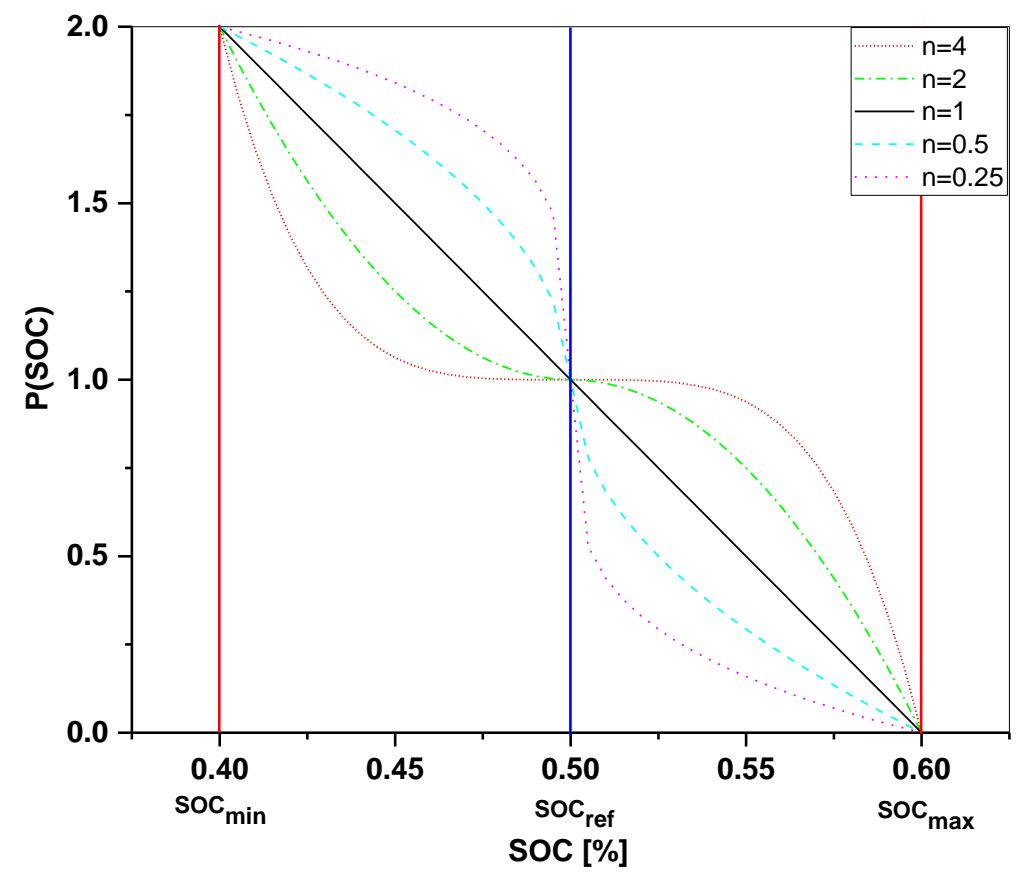

Figure 4.10 Penalty weight used in ECMS to correct the SOC deviation 
From the instantaneous equivalent fuel consumption function Equation 4.25 and the penalty Equation 4.31, the instantaneous equivalent fuel consumption with penalty is expressed as

$$
m_{f, e q u}(t, u)=m_{f}(t, u)+\lambda(t) \cdot P(S O C) \cdot \frac{P_{e l e}(t, u)}{H_{f}}
$$

In the Beijing cycle, the corrected fuel economy $\left(F E_{c o r r}\right)$ and the change in SOC $(\triangle S O C)$ are examined by varying the exponential coefficient $n$ over a range of $n \ni[0,0.25,0.5$, $0.75,1,2,4]$. Figure 4.11 shows variation of the $F E_{c o r r}$ and $\triangle S O C$ simulated using ECMS with different exponential coefficients of penalty equation. A small exponential coefficient $n$ causes high frequency charging/discharging the battery. When $n$ is greater than 0.5 , increasing $n$ leads to a slight increment of $F E_{\text {corr }}$ while causing a significant change of $S O C$. The case with $n=1$ seems to achieve the best result, the better $F E_{c o r r}=$ $10.78 \mathrm{mpg}$ and the smaller $\triangle S O C=1.17 \%$.

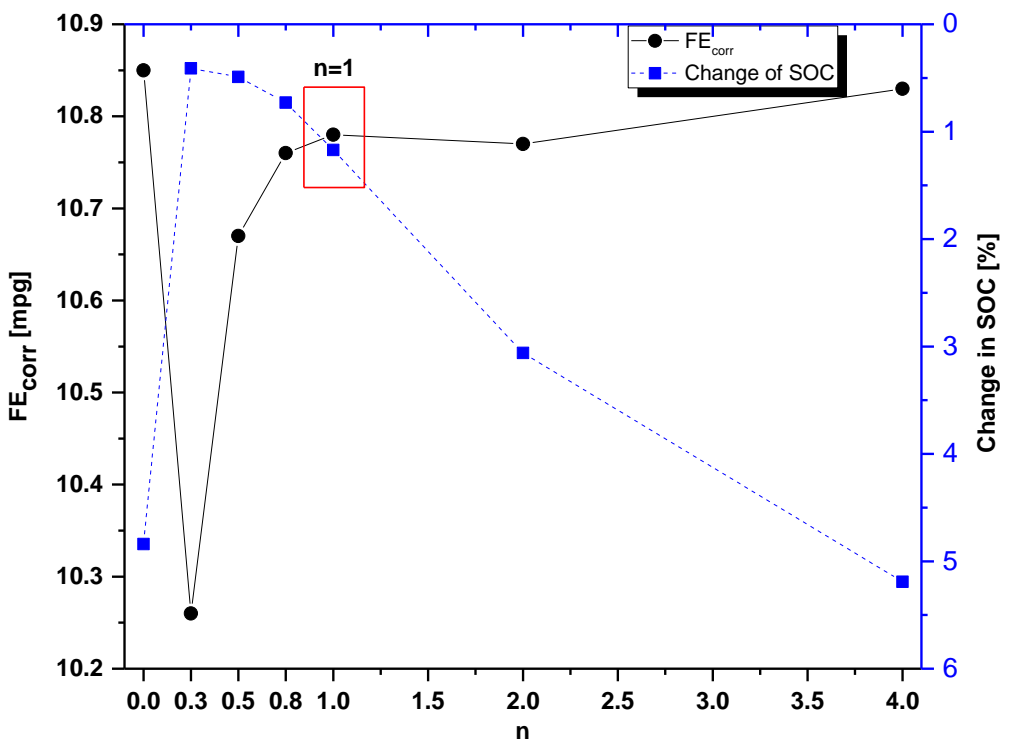

Figure 4.11 The effect of the exponential coefficient on $\mathrm{FE}_{\text {corr }}$ and $\triangle \mathrm{SOC}$

The penalty function added to the ECMS in achieving relatively stable and sustainable SOC is demonstrated in Figure 4.12. It is evident that the SOC is very sensitive to the $\lambda$ 
value selected. The operation of this vehicle with ECMS without penalty function, represented by $\lambda=2.1$ and 2.3 , was not able to maintain sustainable SOC. In comparison, the SOC trajectories observed with the ECMS integrated with the penalty function $\left(\lambda_{0}=\right.$ 2.1 and 2.3) was comparable to that derived using DP and the optimal $\lambda=2.227$.

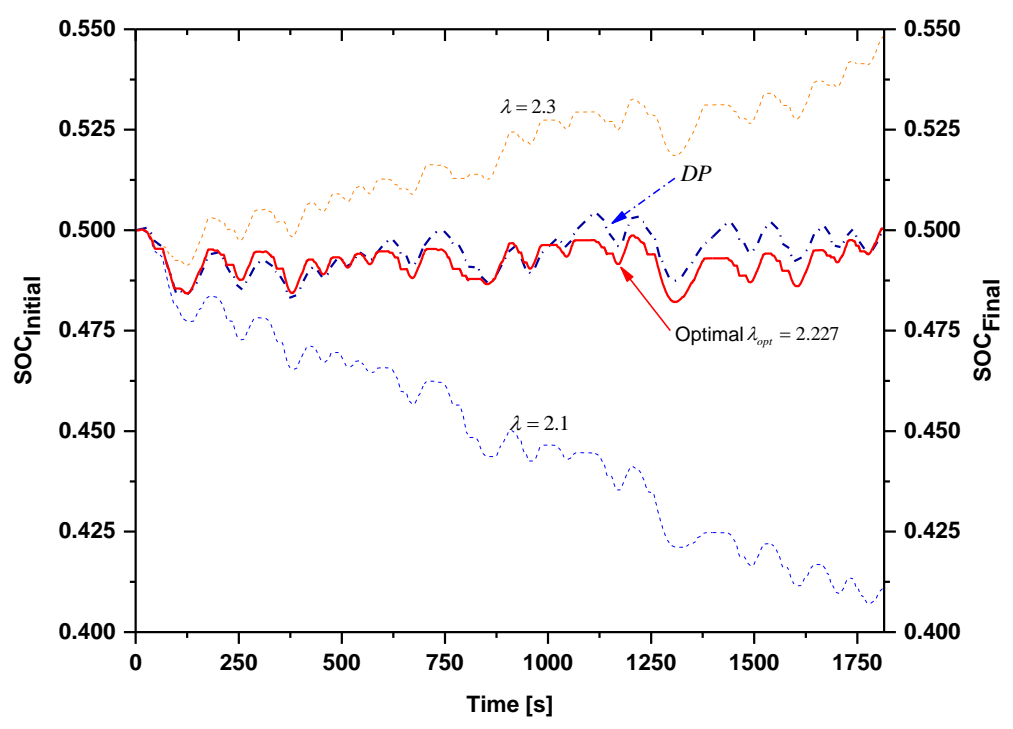

(a) ECMS w/o penalty

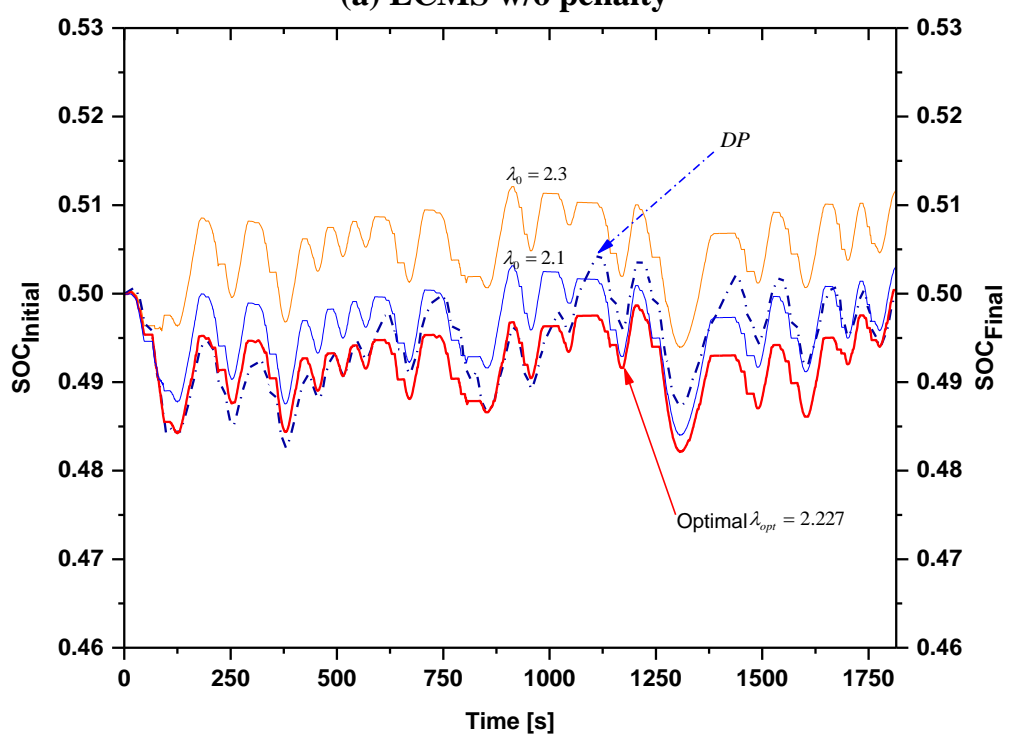

(b) ECMS w/ penalty

Figure 4.12 Comparison of SOC trajectories obtained by DP and ECMS w/ or w/o penalty in the Beijing cycle $(n=1)$

In the penalty function, the selected exponential coefficient (n) is 1 , which can provide sufficient response rates for the penalty weight and get better $F E_{c o r r}$ while ensuring a 
charge-sustainable operation of the battery. The corrected time-varying $\lambda$ trajectory was quickly modified to approach the $\lambda_{\text {opt }}=2.227$ (the red line in Figure 4.13).

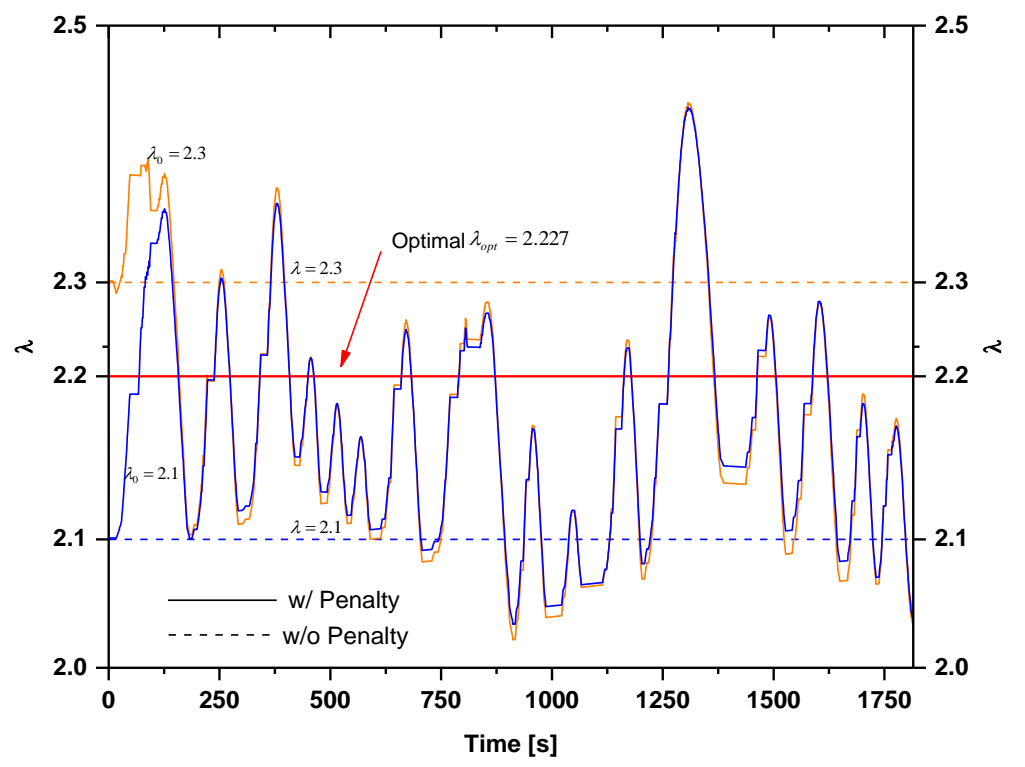

Figure 4.13 Comparison of $\lambda$ trajectories obtained by ECMS w/ or w/o penalty in the Beijing cycle $(n=1)$

In the ECMS without penalty algorithm, it is possible to find a time-invariant $\lambda$ for the perfect charge sustaining ability and FE of parallel HTBs. This value is considered as the optimal equivalent factor $\lambda_{\text {opt }}$ of the ECMS. In the ECMS with penalty algorithm, the corrected $\lambda(t)$ is affected by the variation in SOC. It is important to find a suitable initial value $\lambda_{0}$ for the perfect charge sustaining ability and improved fuel economy.

The optimal equivalent factor $\lambda_{\text {opt }}$ is selected based on the convergence of SOC and the normalized $F E_{\text {corr }}$, which is compared the global optimal solution obtained from DP as shown in Table 4.5.

The ECMS with penalty has been shown to perform well for the reasonable initial $\lambda_{0}=2.1$ and 2.3. As the SOC trajectory stay close to its reference and the simulated $F E_{c o r r}$ results are comparable to that developed using optimal ECMS as shown in Table 4.5 and Figure 4.12. In this research, the optimal initial value $\lambda_{0}$ will be developed using ECMSwDP. 
Table 4.5 Comparison of corrected FE obtained by DP and ECMS w/ or w/o penalty in the Beijing cycle

\begin{tabular}{|c|c|c|c|c|c|}
\hline & $\begin{array}{c}\text { Constant } \boldsymbol{\lambda} \\
\text { and Initial } \boldsymbol{\lambda}_{\mathbf{0}}\end{array}$ & $\mathbf{F E}(\mathbf{m p g})$ & $\boldsymbol{\Delta} \mathbf{S O C}$ & $\mathbf{F E}_{\text {corr }}(\mathbf{m p g})$ & Gap to DP \\
\hline \multirow{2}{*}{$\begin{array}{c}\text { ECMS } \\
\text { w/o penalty }\end{array}$} & $\lambda=2.1$ & 11.22 & $-8.92 \%$ & 9.76 & $12.62 \%$ \\
\cline { 2 - 6 } ECMS & $\lambda=2.3$ & 9.82 & $4.84 \%$ & 10.61 & $5.01 \%$ \\
\hline w/ penalty & $\lambda_{0}=2.1$ & 10.73 & $0.3 \%$ & 10.77 & $3.58 \%$ \\
\cline { 2 - 6 } & $\lambda_{0}=2.3$ & 10.60 & $1.17 \%$ & 10.78 & $3.49 \%$ \\
\hline Optimal ECMS & $\lambda_{\text {opt }}=\mathbf{2 . 2 2 7}$ & $\mathbf{1 0 . 8 0}$ & $\mathbf{- 0 . 0 4 \%}$ & $\mathbf{1 0 . 8 1}$ & $\mathbf{3 . 2 2 \%}$ \\
\hline $\mathbf{D P}(\boldsymbol{\alpha}=\boldsymbol{\beta}=\boldsymbol{\gamma}=\mathbf{0})$ & $\mathbf{-}$ & $\mathbf{1 1 . 1 7}$ & $\mathbf{0 . 0 0}$ & $\mathbf{1 1 . 1 7}$ & $\mathbf{0 . 0 0 \%}$ \\
\hline
\end{tabular}

\subsection{Summary}

This chapter presents the DP and ECMS strategies and their application in improving the performance of a hybrid transit bus in the backward vehicle simulator. The simulation results obtained using DP are the globally optimal solution with the known operation cycle. Although DP cannot be used as on-time controller, the results obtained using DP represent the best performance theoretically achievable and provide the benchmark for the evaluation of the actual controlling approaches. In this research, the cost function of DP is further improved with the penalty terms on engine on/off, motor current and $\mathrm{NO}_{\mathrm{x}}$ emissions for the benefit achieved by the proposed less frequent on/off operation of the engine, motor and $\mathrm{NO}_{\mathrm{x}}$ emissions reduction.

The ECMS was further improved by adding the penalty function associated with the deviation in SOC by adjusting the $\lambda$ based on the SOC variation of battery without considering the driving condition and the selection of initial $\lambda_{0}$. Such a modification enhances the robustness of the strategy development of the energy management controller. The development of the optimal $\lambda$ (which is a function of SOC, vehicle operation features, or route information) will be investigated in the next chapter. 


\section{ECMSwDP}

As previously described, the optimization of a HEV control strategy is a dynamic optimal problem involving a nonlinear cost function and nonlinear constraints. DP is capable of dealing with a nonlinear non-convex optimization problem and finding the globally optimal solution. However, the optimization of HEV operation using DP requires the pre-known driving cycle and heavy calculation cost. Accordingly, DP cannot be used in the real-time optimal controller. ECMS is a real-time control approach but always results in local optimal solution because of the non-convex cost function. To take the advantage of the global optimality of DP and the real-time capability of ECMS as show in Figure 5.1, one ECMS with DP combined model (ECMSwDP) was proposed. In this approach, the optimal $\lambda$ for ECMS can be derived using DP under various driving conditions and terrains of future transit bus routes. The $\lambda$ derived using DP will be used in a real-time ECMS controller to achieve the best compromise between the FE and exhaust emissions of a hybrid electric transit bus.

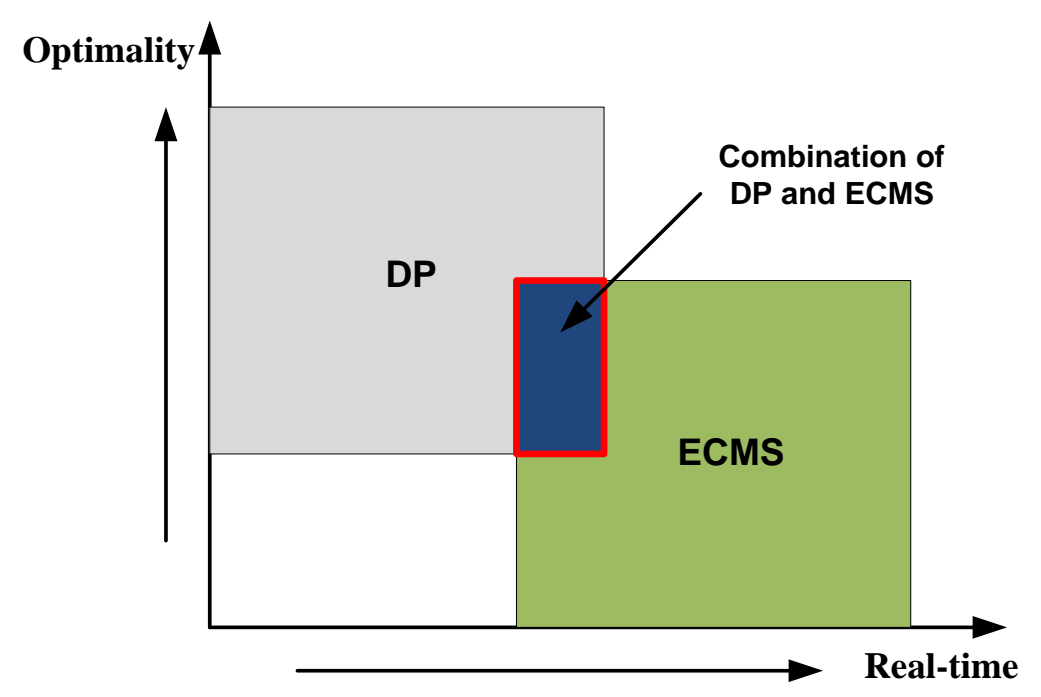

Figure 5.1 The benefit from the combination of DP and ECMS 


\subsection{The ECMSwDP Algorithm}

In the ECMSwDP algorithm, DP minimizes the equivalent cost function of the ECMS presented in Equation 4.25. The known driving power requested is used to derive the optimal time-varying $\lambda$ trajectory noted as $\lambda(t)$. This algorithm will promote the local optimal solution from ECMS toward the global optimal solution of DP.

The equivalent cost of ECMSwDP is parameterized by $\left(m_{f}, P_{\text {ele }}\right)$, which replaced the engine operation variables $\left(T_{i c e}, \omega_{i c e}\right)$ used in DP operation. The fuel rate is calculated using the brake specific fuel consumption (BSFC), which is a function of engine torque $T_{\text {ice }}$ and engine speed $\omega_{\text {ice }}$ presented in the Equation 3.1.

Also, for any time-variant $\lambda(t)$, we can find an optimal and feasible engine operation point $\left(T_{\text {ice }}, \omega_{\text {ice }}\right.$ ) by solving the equivalent cost function. The fuel consumption $m_{f}$ is derived from the engine map, and the $P_{e l e}$ is calculated using battery power equation presented in Equation 3.5.

The control variable of ECMSwDP is the equivalent factor $\lambda$ instead of engine torque $T_{\text {ice }}$ as in the original DP. At the same time, the feasible engine and motor operation points should follow the inequality constraints from Equation 4.7 to Equation 4.10:

The variables and grids of ECMSwDP algorithm have been shown in Table 5.1. The control grid $\mathrm{P}$ varies between the maximum $\left(\lambda_{\max }\right)$ and minimum $\left(\lambda_{\min }\right)$ equivalent factor, which is defined as $P \in\left[\lambda_{\min }, \lambda_{\min }+0.1, \cdots \lambda_{\max }\right]$, where the step size of the control variable $d(\lambda)$ is 0.1 . The initial and final SOC are set to 0.5 , the quantization increment of the SOC and time step is set to $0.01 \%$ and $1 \mathrm{~s}$, respectively. The engine brake power is accounted for by setting the minimum engine torque as $-100 \mathrm{Nm}$. 
Table 5.1 Variables and grids of ECMSwDP algorithm

\begin{tabular}{|c|c|c|}
\hline & Variables & Grid \\
\hline Stage & Time $[\mathrm{s}]$ & $\mathrm{N} \in[0: 1:$ End $]$ \\
\hline Control & $\lambda[-]$ & $P \in\left[\lambda_{\min }: 0.1: \lambda_{\max }\right]$ \\
\hline State & SOC $[-]$ & $\mathrm{L} \in[0.4: 0.0001: 0.6]$ \\
\hline
\end{tabular}

The ECMSwDP model calculates the optimal ECMS cost $J(k, \lambda)$ at each time step $k$ of the control grid $P$, and the corresponding databases $\left\{T_{i c e}(k, \lambda), T_{e m}(k, \lambda), m_{f}(k, \lambda)\right.$, $\left.\omega_{\text {ice }}(k, \lambda), \omega_{\text {em }}(k, \lambda)\right\}$ are calculated and stored. For example, Figure 5.2 shows the calculated $\lambda$ database for the Beijing cycle. It is possible to approximate the optimal costate of the ECMS based on realistic data rather than heuristic information. For each control variable $\lambda(k) \in P$, the corresponding $\left\{m_{f}(k), S O C(k),\right\}$ can be calculated by the one to one relationship databases given by in Equation 3.1 and Equation 3.7. After that, following the same progress as the DP algorithm, the ECMSwDP model calculates and stores the sequence of the optimal powertrain operation $\left(T_{i c e}, T_{e m}, \omega_{i c e}, \omega_{e m}\right)$ and the corresponding control variables $\lambda(t)$, which is the optimal $\lambda$ based on the battery SOC and driving condition. The calculation procedures for implementing ECMSwDP to the parallel HEV is shown in Figure 5.3.

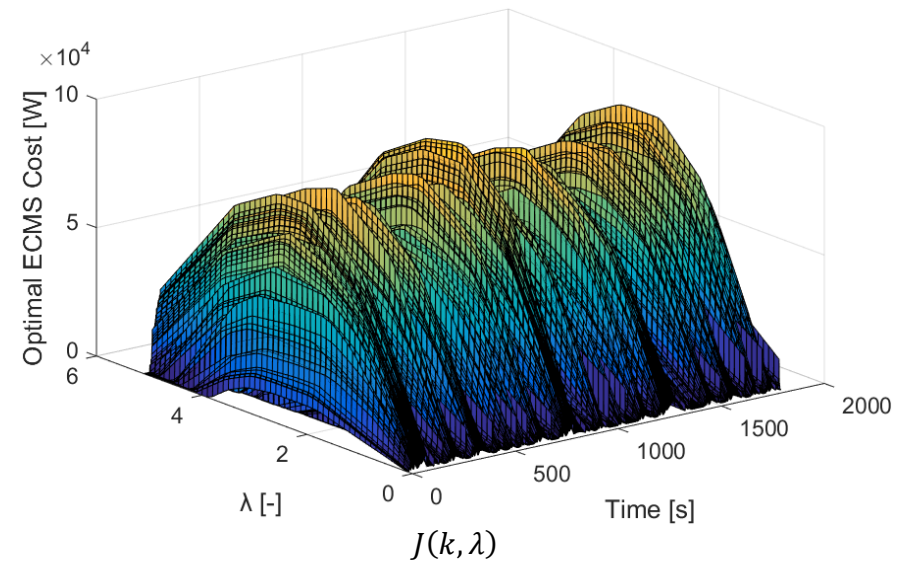



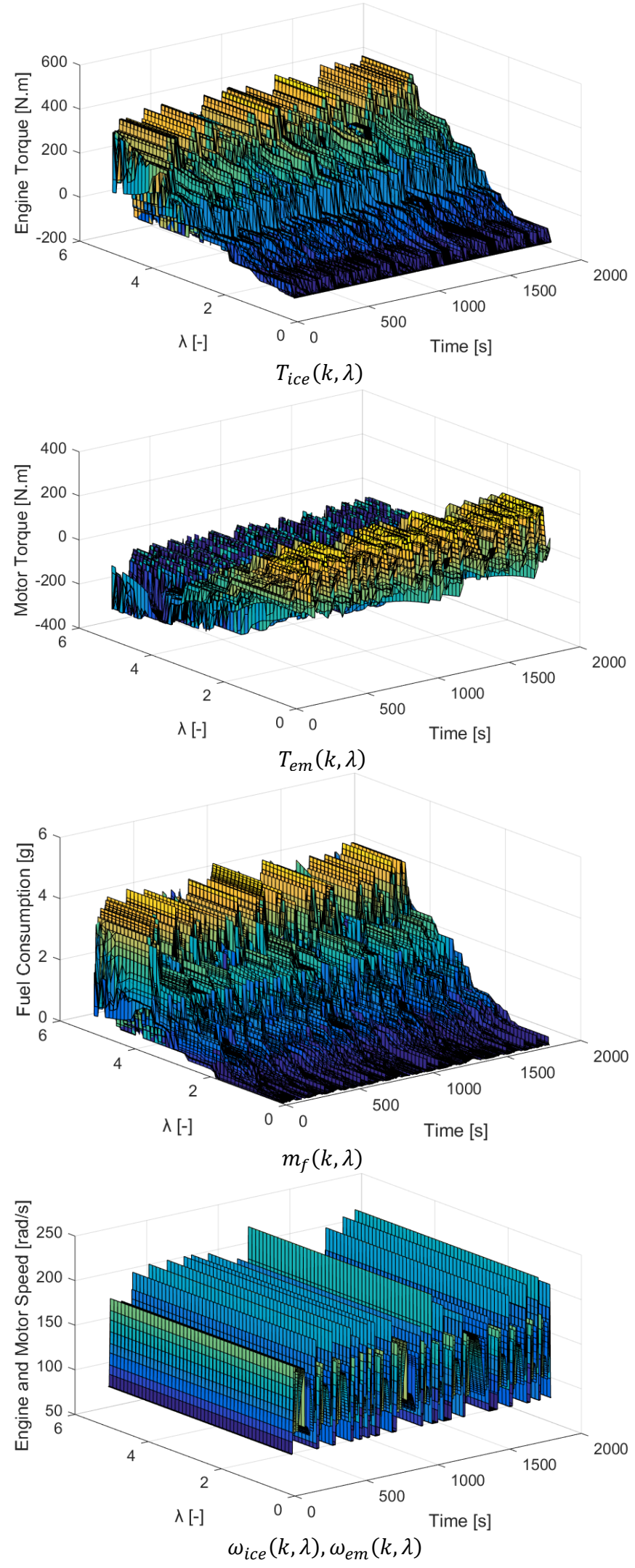

Figure 5.2 The $\lambda$ corresponding databases of the ECMS cost (Beijing cycle) 


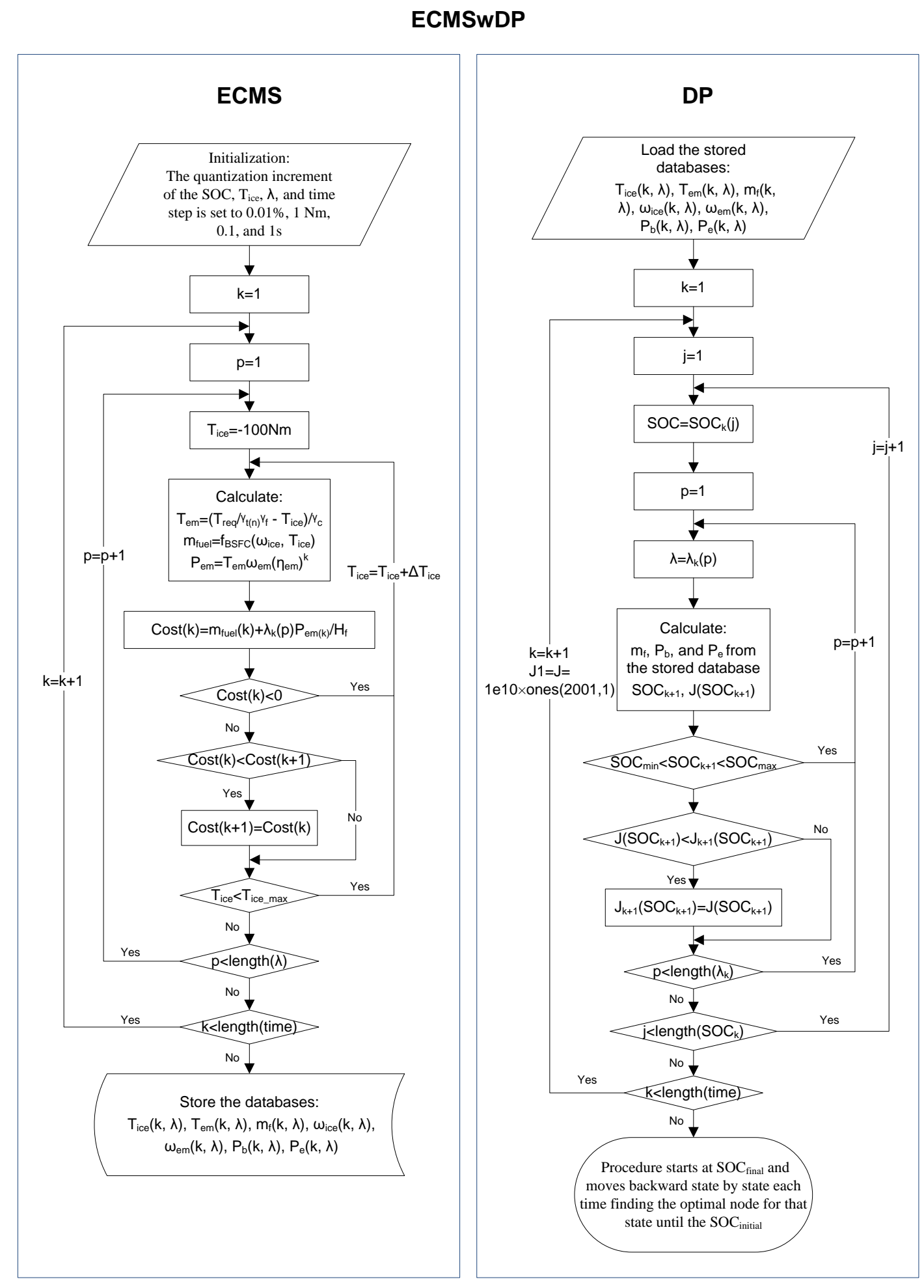

Figure 5.3 Flow chart of ECMSwDP procedures 


\subsection{Simulation Results and Discussions}

This subsection implements the ECMSwDP described in the previous section to extract the optimal $\lambda$ and develop the $\lambda$ lookup table for an ECMS controller. The simulated performance with ECMSwDP is compared to the globally optimal solution developed using DP. The effect of road grade and $\lambda$ range on vehicle performance with ECMSwDP is examined and discussed. Similar to that of the DP and ECMS model, the ECMSwDP simulator primarily uses a simplified quasi-static map in the pre-transmission parallel HEV model, which was developed in the MATLAB/ m script environment.

\subsubsection{The Extraction of Time-Variant $\lambda(t)$ using the ECMSwDP Model}

In ECMSwDP model, DP minimizes the equivalent cost function of the ECMS showed in Equation 4.25 from the fixed route with known driving information to derive the optimal time-variant $\lambda(t)$ trajectory. Figure 5.3 shows the the variation of the SOC and optimal $\lambda(t)$ trajectory derived using ECMSwDP with or without the improved cost function over the Beijing cycle. In order to further validate the performance of the optimal ECMSwDP for different driving conditions, the WVU-CSI cycle was chosen as another testing cycle. The simulation results are shown in the Appendix C.

As shown in Figure 5.4, the $\lambda(t)$ values are very sensitive to the change of the SOC. At the beginning of cycle, $\lambda(t)$ is very low, indicating the preference of using electrical energy. This is consistent with the gradually decreasing SOC. The consumption of electrical energy prior to deceleration after $100 \mathrm{~s}$ discharges the battery and makes it available to absorb the electrical energy recovered during the coming braking process or the energy from the ICE. As shown in Figure 5.4, the $\lambda(t)$ values observed between 100s to 180 s is relatively high indicating the preference of charging the battery with electricity recovered during the braking process. This is consistent with the corresponding increase in SOC. However, the 
$\lambda(t)$ values at the beginning of the WVU-CSI cycle showed in Figure C. 1 are high, indicating the preference of using engine power to charge the battery for the upcoming hard driving condition. The ECMSwDP model assumes that all kinetic energy is either consumed due to the power loss or recovered by the brake energy recovery system without involving mechanical brake.

As shown in Figure 5.4, the fluctuation of the SOC trajectory derived with improved cost function is relatively smooth compared to that without the improved cost function. Accordingly, the engine delivers less work in charging battery for reducing $\mathrm{NO}_{\mathrm{x}}$ emissions when the improved cost function is applied.

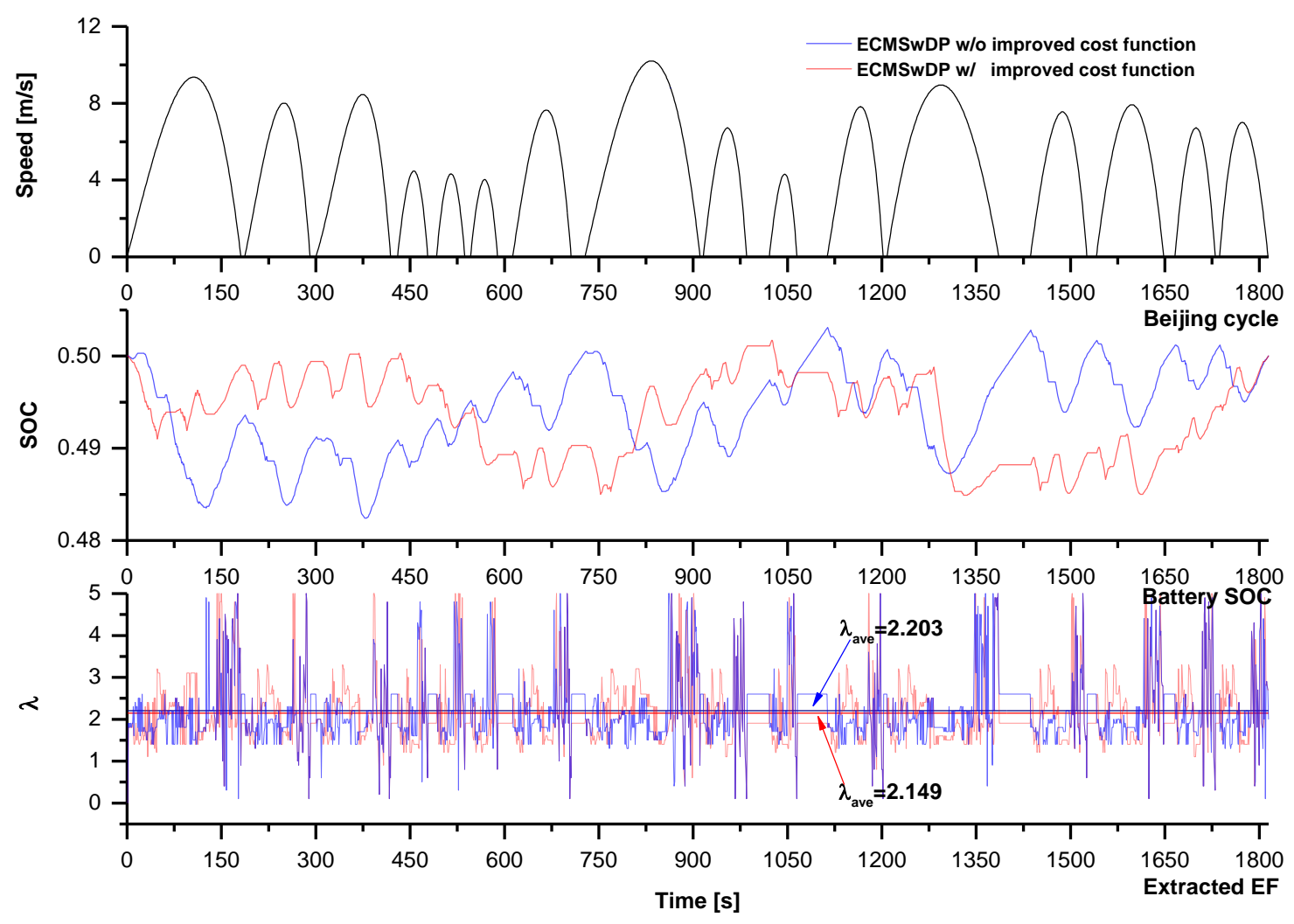

Figure 5.4 The extracted time-variant $\lambda(t)$ obtained by ECMSwDP with or without the improved cost function over the Beijing cycle

Also, the significant change in the $\lambda(t)$ trajectory observed with the improved cost function is less frequent (still vary a lot but less frequent) than that without the improved cost 
function. The operation of the engine and EM is relatively steady with the improved cost function. The average value of $\lambda$ extracted with improved cost function $\left(\lambda_{\text {ave }, w / i}=\right.$ $2.149)$ is smaller than the one without the improved cost function penalty $\left(\lambda_{\text {ave,w/o } i}=\right.$ 2.203), indicating the ECMSwDP with the improved cost function tends not to charge battery using engine power, which reduces $\mathrm{NO}_{\mathrm{x}}$ emissions.

It is noted that the $\lambda_{a v e, w / i}=2.149$ and $\lambda_{a v e, w / o i}=2.203$ are very close to the optimal $\lambda$ value $\lambda_{\text {opt }}=2.227$ derived using the ECMS as presented in Chapter 4 . It seems that the average of the $\lambda(t)$ derived using ECMSwDP can be considered as the optimal $\lambda$ described in Chapter 4.

\subsubsection{The Study of ECMS with the Extracted $\lambda(t)$}

In this session, the $\lambda(t)$ extracted using the ECMSwDP model was verified as the optimal one for the ECMS algorithm. Figure 5.5 compares the Beijing cycle performance of this hybrid bus operated with ECMS with $\lambda(t)$ derived using ECMSwDP.

The variation of $S O C, P_{e}$, and $P_{b}$ observed using ECMS with $\lambda(t)$ extracted using ECMSwDP are comparable to those observed with DP. The corrected fuel economy observed using ECMS with the $\lambda(t)$ extracted using ECMSwDP is $10.80 \mathrm{mpg}$, which is comparable to the $11.17 \mathrm{mpg}$ observed using DP. As shown in Table 5.2, the difference in the changes in SOC observed in these two cases is very small. As shown in Figure 5.6, the distribution of the engine and electric motor operation points are also similar. 


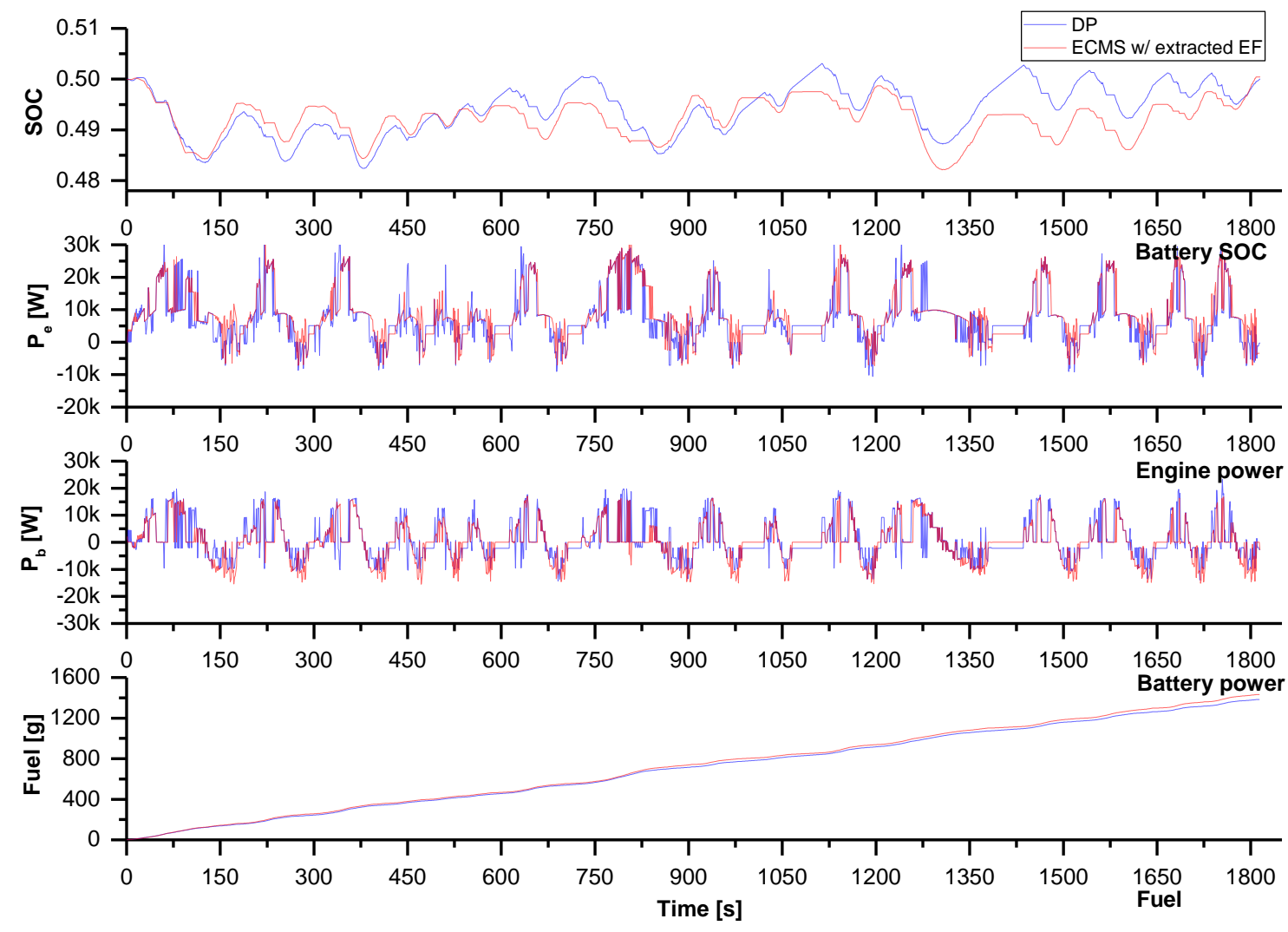

Figure 5.5 The comparison between the ECMS with the extracted $\lambda(t)$ and DP without the improved cost function over the Beijing cycle

Table 5.2 Comparison of the ECMS with the extracted $\lambda(t)$ and DP $(\alpha=\beta=\gamma=0)$ simulation results in the Beijing cycle

\begin{tabular}{|c|c|c|c|c|}
\hline & FE(mpg) & $\Delta$ SOC & FE $_{\text {corr }}(\mathbf{m p g})$ & Gap to DP \\
\hline ECMS w/ the extracted $\lambda(\boldsymbol{t})$ & 10.79 & $0.01 \%$ & 10.80 & $3.12 \%$ \\
\hline $\mathbf{D P}(\boldsymbol{\alpha}=\boldsymbol{\beta}=\boldsymbol{\gamma}=\mathbf{0})$ & $\mathbf{1 1 . 1 7}$ & $\mathbf{0 . 0 0 \%}$ & $\mathbf{1 1 . 1 7}$ & $\mathbf{0 . 0 0 \%}$ \\
\hline
\end{tabular}




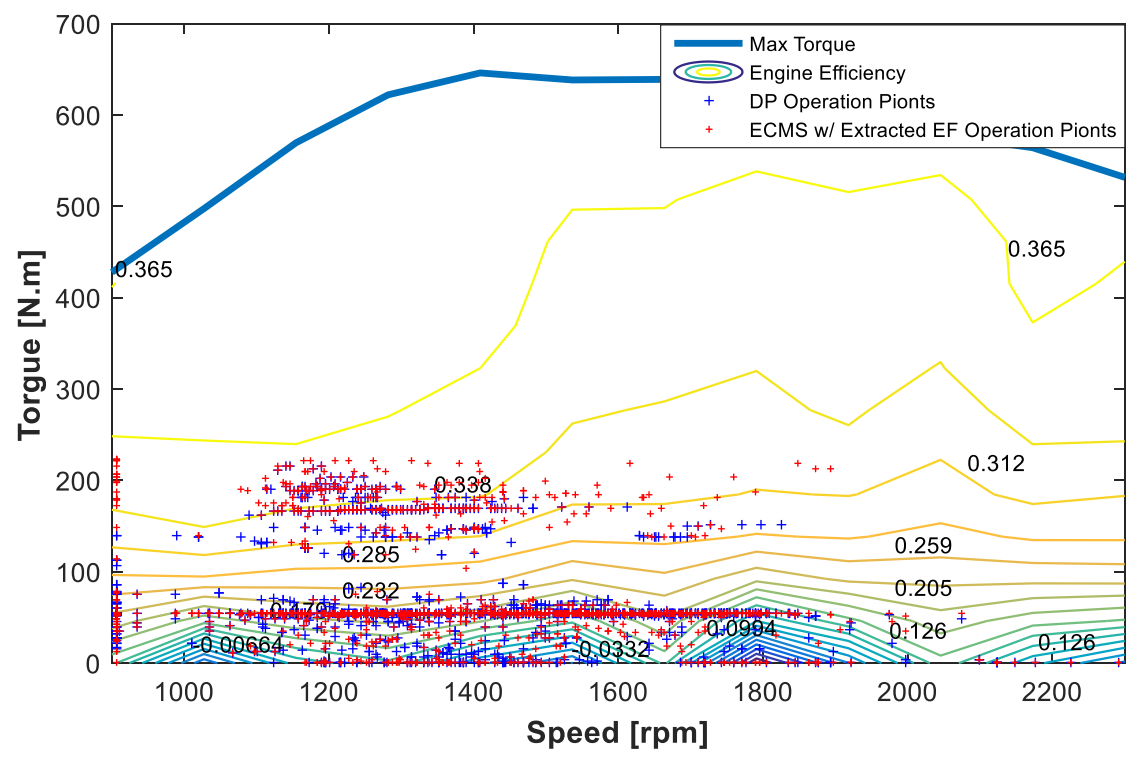

(a) Engine operation points distribution

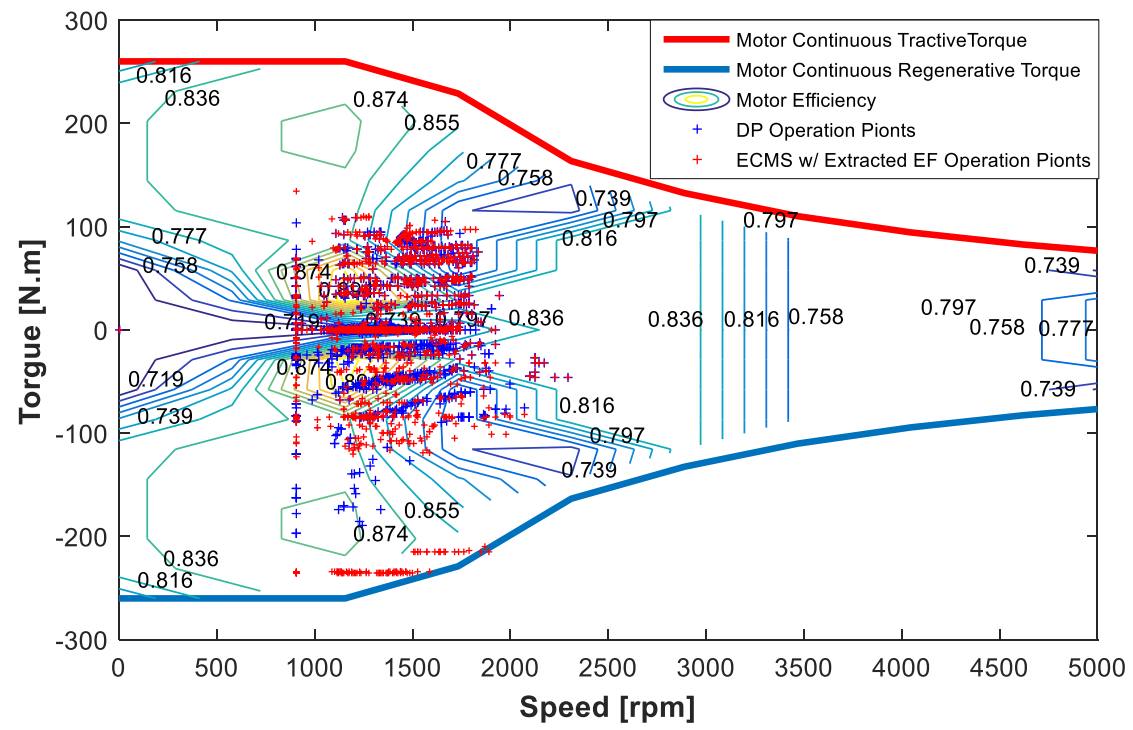

(b) Electric Motor operation points distribution

Figure 5.6 ECMS with the extracted $\lambda(t)$ and DP power source operation points distribution in maps (Beijing cycle)

\subsubsection{The Study of ECMS Based on the Average of the $\lambda$ Extracted Using ECMSwDP}

As previously described in Chapter 4 (Equation 4.27), the $\lambda$ can be approximated as a constant for the known driving condition when the changes in the SOC of the battery can be ignored. The optimal constant $\lambda$ (noted as $\lambda_{\text {opt }}$ ) is usually derived based on the 
minimum $F E_{\text {corr }}$ simulated while satisfying the battery charge sustainability. However, this makes it very difficult for the real-time controller to derive the $\lambda_{\text {opt }}$ without knowing the actual vehicle operations or predicted operation characteristics.
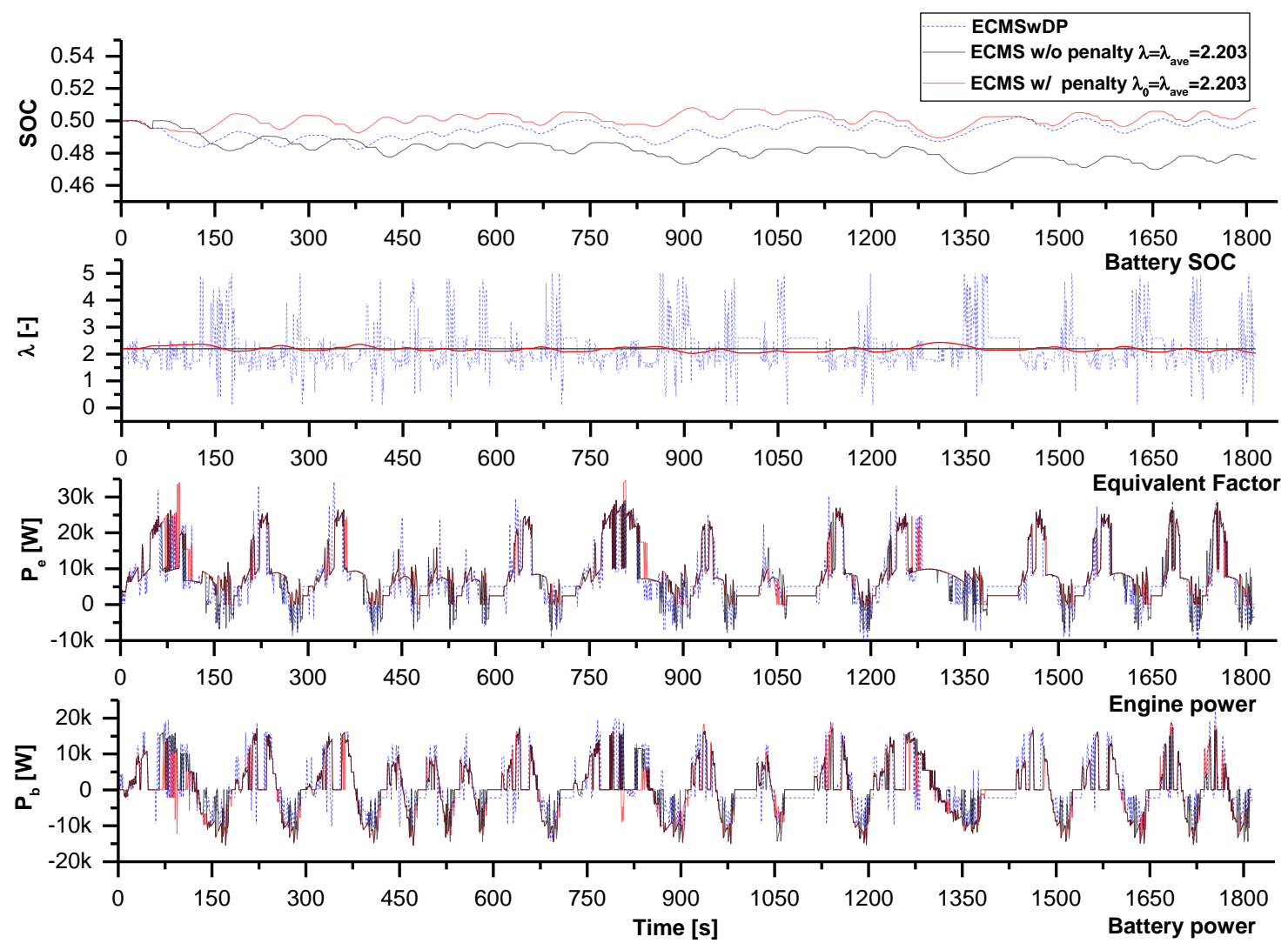

Figure 5.7 The variation in SOC, $\lambda, P_{e}, P_{b}$ over the Beijing cycle simulated with ECMS w/ and w/o penalty function, and DP

In this research, the time variant $\lambda(t)$ developed using ECMSwDP may provide a new method for the selection of the optimal constant value $\lambda_{o p t}$. As mentioned in the prior section, the averages of the $\lambda(t)$ derived using ECMSwDP with or without improved cost function $\left(\lambda_{\text {ave }, w / i}=2.149\right.$ and $\left.\lambda_{\text {ave }, w / o i}=2.203\right)$ are very close to the optimal $\lambda$ value $\left(\lambda_{\text {opt }}=2.227\right)$. Accordingly, the average of $\lambda$ extracted using ECMSwDP may be considered as approximate $\lambda_{o p t}$ in the ECMS. Such an assumption will be demonstrated 
as valid by comparing the performance of the HEB operated with ECMSwDP, and ECMS w/ and w/o the penalty function as presented in Chapter 7.

The average $\lambda$ derived without accounting for the improved cost function noted as $\lambda_{\text {ave } w / o i}=2.203$ was used as example. The impact of the ECMS w/ and w/o penalty function in satisfying the battery charge sustainability is also examined. Figure 5.7 shows the performance of this HEB over the Beijing cycle observed with ECMSwDP, ECMS without the penalty function represented by a constant $\lambda\left(\lambda_{\text {ave }}=2.203\right)$, and ECMS with the penalty function represented by the initial $\lambda_{0}\left(\lambda_{\text {ave }}=2.203\right)$. The operation of this HEB over the Beijing Cycle with ECMS without the penalty function represented by a constant $\lambda\left(\lambda=\lambda_{\text {ave }}=2.203\right)$ was not able to achieve the charge sustainable operation of battery. In comparison, the application of the penalty function makes it possible for ECMS to achieve the battery charge sustainability when the average $\lambda$ derived by ECMSwDP is considered as the optimal initial $\lambda$ noted as $\lambda_{0}\left(\lambda_{0}=\lambda_{\text {ave }}=2.203\right)$.

Table 5.3 compares the fuel economy achieved in the three cases simulated. The corrected fuel economy of this HEB using ECMS with penalty function was $10.78 \mathrm{mpg}$, which was $3.49 \%$ gap to the best fuel economy (11.17 mpg) achieved using DP. Accordingly, the average extracted $\lambda$ value derived from ECMSwDP can be set as the appropriate initial value $\lambda_{0}$ of the ECMS with penalty.

Table 5.3 The simulation results of the ECMS with or without penalty function and DP in the Beijing cycle

\begin{tabular}{|c|c|c|c|c|c|}
\hline & $\boldsymbol{\lambda}$ & $\mathbf{F E}(\mathbf{m p g})$ & $\boldsymbol{\Delta S O C}$ & $\mathbf{F E}_{\text {corr }}(\mathbf{m p g})$ & Gap to DP \\
\hline Rule-based & - & 7.05 & $-0.10 \%$ & 7.05 & $36.88 \%$ \\
\hline ECMS w/o penalty & $\lambda=\lambda_{\text {ave }}=2.203$ & 11.06 & $-1.81 \%$ & 10.56 & $5.46 \%$ \\
\hline ECMS w/ penalty & $\lambda=\lambda_{\text {ave }}=2.203$ & 10.66 & $0.78 \%$ & 10.78 & $3.49 \%$ \\
\hline $\mathbf{D P}(\boldsymbol{\alpha}=\boldsymbol{\beta}=\boldsymbol{\gamma}=\mathbf{0})$ & - & $\mathbf{1 1 . 1 7}$ & $\mathbf{0 . 0 0 \%}$ & $\mathbf{1 1 . 1 7}$ & $\mathbf{0 . 0 0 \%}$ \\
\hline
\end{tabular}

This was also demonstrated using the WVU-CSI cycle as an example using the $\lambda\left(\lambda_{\text {ave }}=\right.$ 2.115) extracted using ECMSwDP as the initial $\lambda_{0}$. As shown in Table 5.4, the corrected 
fuel economy of this HEB over the WVU-CSI cycle observed with ECMS w/ penalty using the average $\lambda$ extracted using ECMSwDP as the initial $\lambda_{o}=2.115$ was $10.66 \mathrm{mpg}$. There is $9.51 \%$ gap to the best fuel economy achieved using DP. The detailed information is presented in Appendix C.

Table 5.4 The simulation results of the ECMS with or without penalty function and DP in the WVU-CSI cycle

\begin{tabular}{|c|c|c|c|c|c|}
\hline & $\boldsymbol{\lambda}$ & $\mathbf{F E}(\mathbf{m p g})$ & $\mathbf{\Delta S O C} \%$ & $\mathbf{F E}_{\text {corr }}$ (mpg) & Gap to DP \\
\hline Rule-based & - & 8.74 & -0.60 & 8.61 & $26.91 \%$ \\
\hline ECMS w/o penalty & $\lambda=\lambda_{\text {ave }}=\mathbf{2 . 1 1 5}$ & 11.89 & -9.67 & 10.24 & $13.07 \%$ \\
\hline ECMS w/ penalty & $\lambda=\lambda_{\text {ave }}=\mathbf{2 . 1 1 5}$ & 10.65 & 0.17 & 10.66 & $9.51 \%$ \\
\hline DP( $\alpha=\boldsymbol{\beta}=\boldsymbol{\gamma}=\mathbf{0})$ & - & $\mathbf{1 1 . 7 8}$ & $\mathbf{0 . 0 0}$ & $\mathbf{1 1 . 7 8}$ & $\mathbf{0 . 0 0 \%}$ \\
\hline
\end{tabular}

\subsubsection{Effect of Road Grade on the ECMSwDP}

Leon (2011) and Khan (2009) proposed to characterize hypothetical road grade using a sine wave and the grade can be assumed as a function of cycle distance. The sinusoidal grade function can be expressed as:

$$
\text { Grade }=G_{m} \sin \left(2 \pi \cdot \frac{s-D_{P}}{D_{t}}\right)
$$

Equation 5.1

Where: Grade is the function output of the current road grade, $G_{m}$ is the maximum/minimum road grade, $D_{t}$ is the total distance of the drive cycle, $s$ is the function input distance, and $D_{P} \in\left[0, D_{t}\right]$ is the phase shift of the wave. The elevation is expressed as function

$$
E=\int_{0}^{D_{t}} \sin \alpha d s
$$

Equation 5.2

where $\alpha=\arctan ($ Grade $/ 100)$.

Figure 5.8 shows an example of the grade added to the Beijing Cycle. The effect of road grade on the $\lambda$ and SOC is also examined with an assumed constant speed (14 mph) operation over a mile-long route with significant variation in elevation, as shown in Figure 
5.9. The variation of elevation with distance is assumed as a sine function where the sine wave maximum grade varied from from $1 \%$ to $10 \%$. The feasible variable values are maximum road grade $G \in\{1 \%, 2 \%, \cdots 10 \%\}$, traveled distance $D_{t}=1$ mile and phase shift $D_{P}=0$, respectively. The model developed in this research is applied to examine the impact of road grade on $\lambda$ of this vehicle operated with ECMSwDP. The ECMSwDP model assumes that all kinetic energy is either consumed due to the power loss or recovered by the brake energy recovery system without involving mechanical brake.

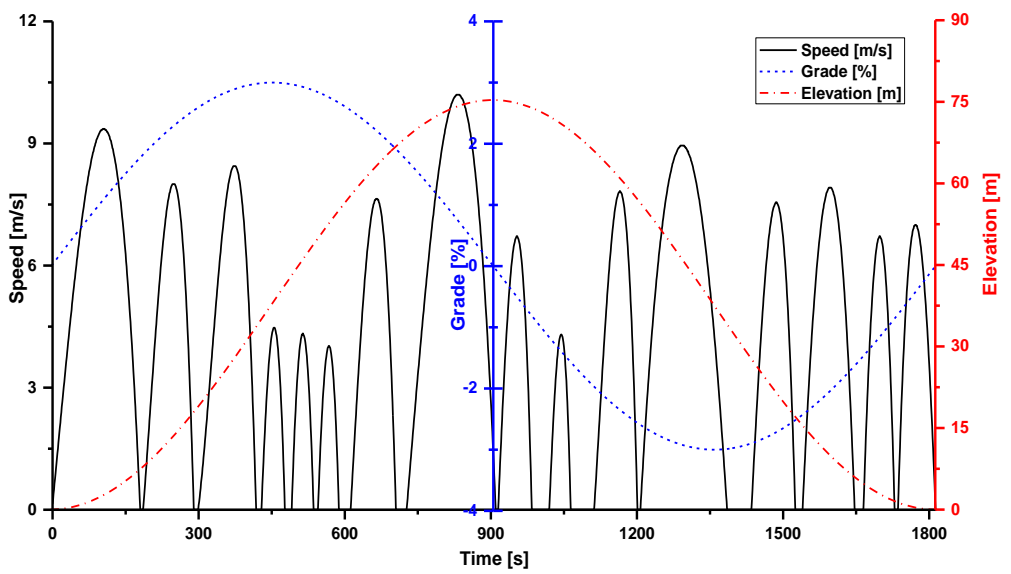

Figure 5.8 Beijing cycle sin wave function and elevation $G_{m}=3 \% \quad D_{p} / D_{t}=0$

Figure 5.10 shows the SOC trajectories obtained using ECMSwDP when the maximum grades of the sine wave are varied from $1 \%$ to $10 \%$. Increasing the maximum grade from $1 \%$ to $10 \%$, the minimum SOC observed prior the maximum grade decreased from 0.493 to 0.415 as shown in Figure 5.11. The battery is discharged with the extent of discharge decided based on the upcoming downhill grade. Increasing the grade will further decrease the mimumum SOC of the battery trajectories. The higher the grade, the more battery energy is discharged in preparation for absorbing brake recovery energy of the downhill terrain. 


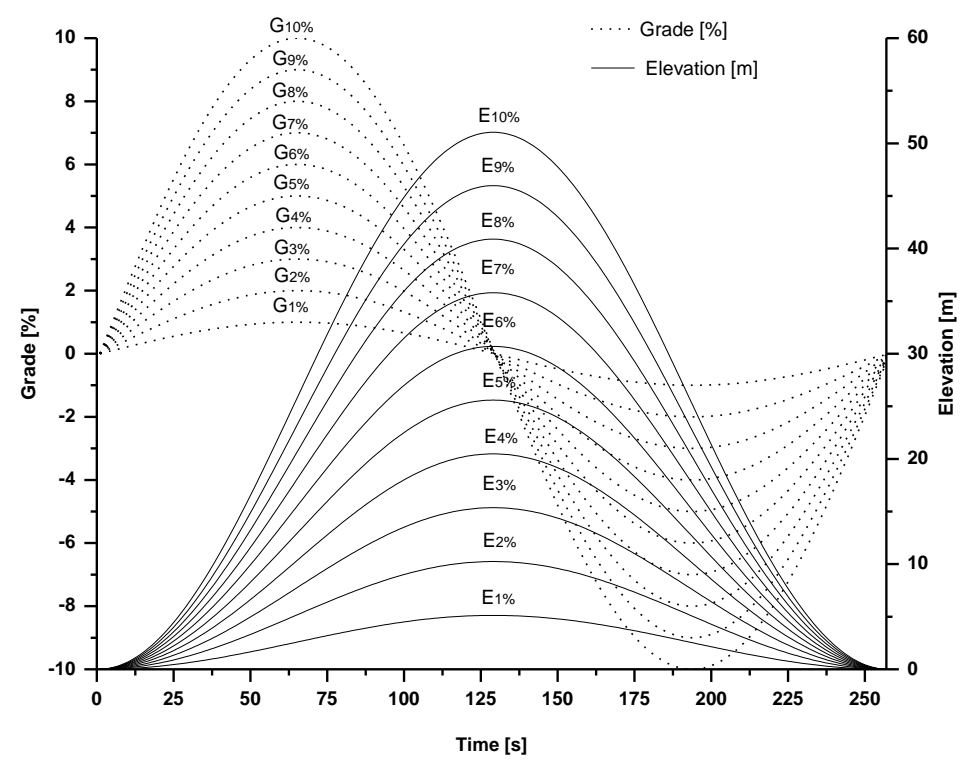

Figure 5.9 Synthetic constant speed with sine wave maximum grade from $1 \%$ to $10 \%$

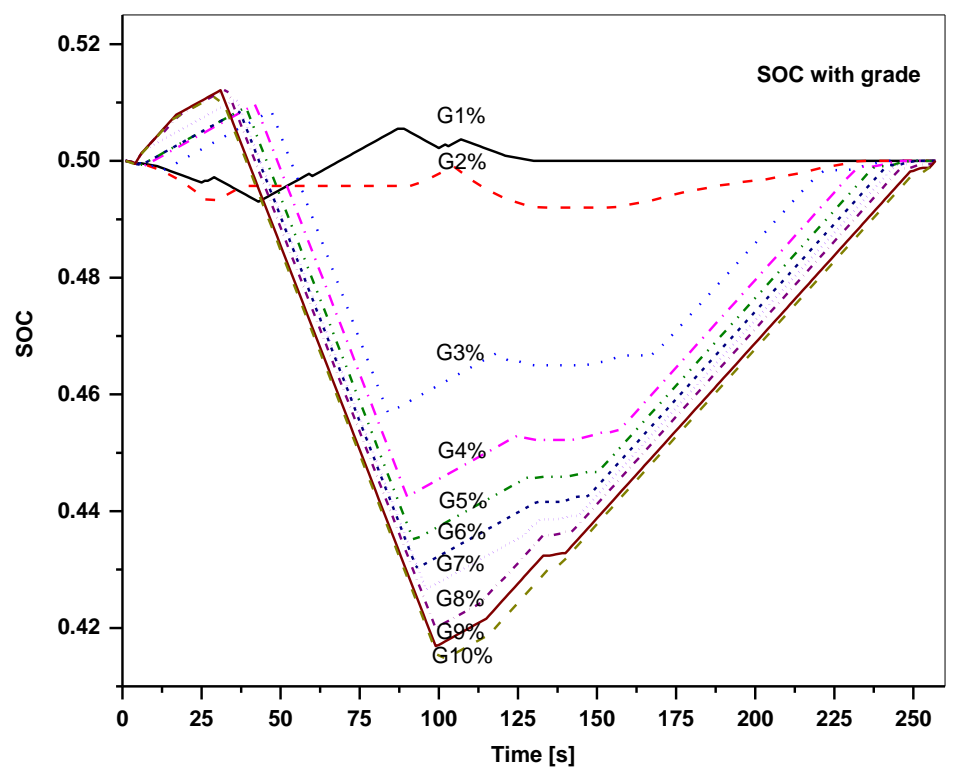

Figure 5.10 Comparison of SOC trajectories obtained by ECMSwDP over the synthetic driving cycle 


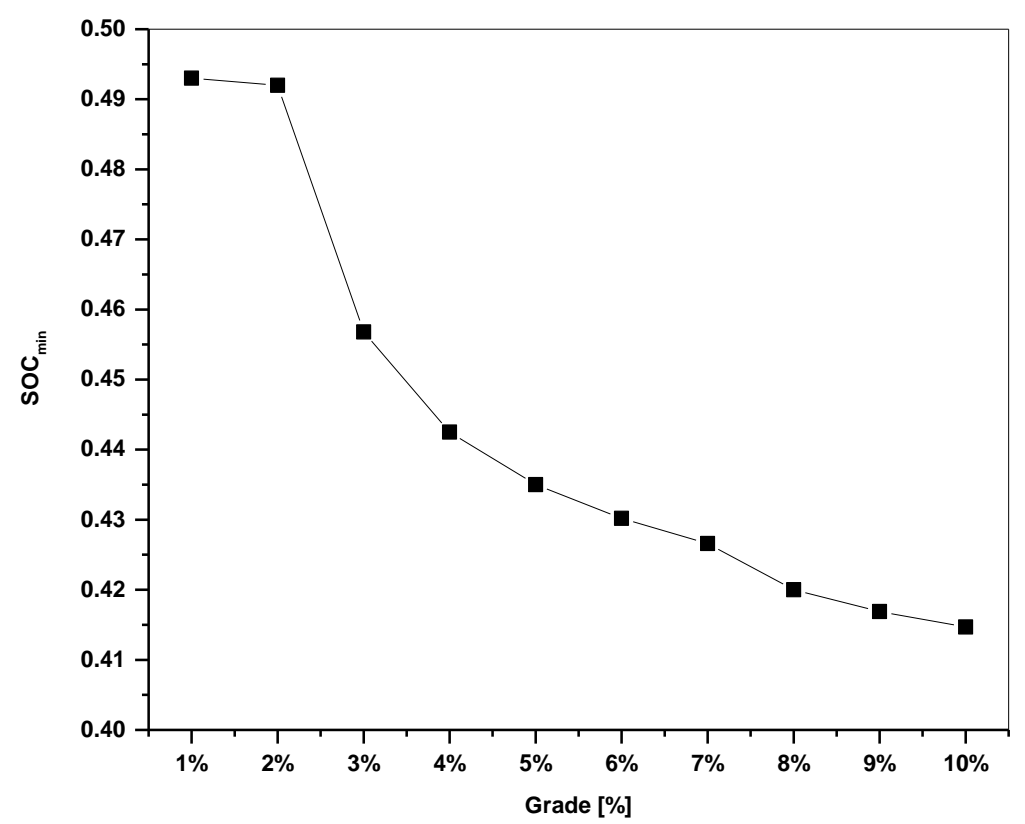

Figure 5.11 The relationship between the minimum SOC and the grade by ECMSwDP over synthetic driving cycle

Figure 5.12 shows the $\lambda$ trajectories obtained using ECMSwDP. Over a sine wave with a maximum grade from $1 \%$ to $10 \%$, the $\lambda$ range is $\lambda \ni[2,4]$. The lowest $\lambda$ value observed from 50s to $100 \mathrm{~s}$ is 2 , which makes the controller discharge the battery so it can store the electrical energy to be recovered during the upcoming downhill terrain. When compared to the rule-based simulation results, the ECMSwDP algorithm improved the fuel economy by $48.7 \%$. The highest improvement was observed with the maximum grade of $10 \%$. This is due to the extra buffer in the battery on the top of the hill in anticipation of capturing regeneration energy. 


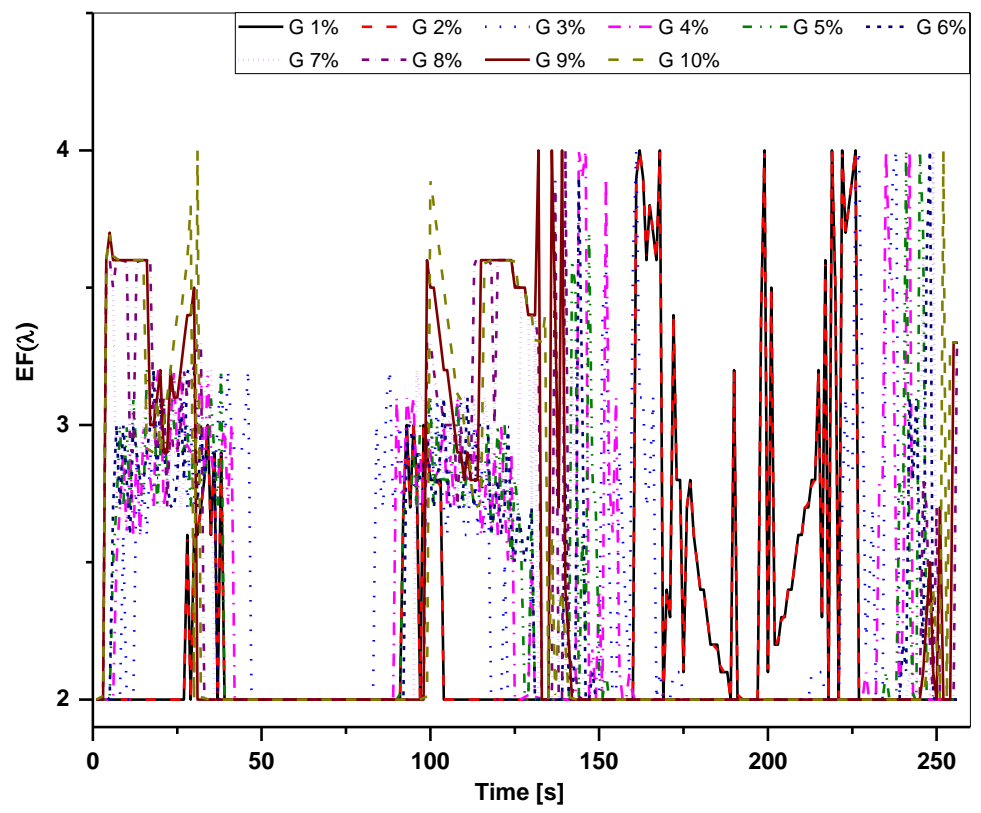

Figure 5.12 Comparison of $\lambda$ trajectories obtained by ECMSwDP over a sine wave with a maximum grade from $1 \%$ to $10 \%$ and a cruising speed of $14 \mathrm{mpg}$

\subsubsection{The Effect of $\lambda$ Range on the ECMSwDP}

The equivalence factor $\lambda$ plays a crucial role in the ECMS. The electrical energy consumed is weighted by the equivalence factor $\lambda$, which is affected by the varied overall efficiencies of the electrical and thermal paths, the battery SOC, and the driving condition. The change of the $\lambda$ range will affect the tradeoff, and accordingly affect the $\mathrm{FE}$ and $\mathrm{NO}_{\mathrm{x}}$ emissions simulated. As previously described, the control variable of the ECMSwDP algorithm is the equivalent factor $\lambda$ instead of engine torque $T_{i c e}$, and equivalent factor grid $\mathrm{P}$ is defined as $P \in\left[\lambda_{\min }, \lambda_{\min }+0.1, \cdots \lambda_{\max }\right]$. The $\lambda$ range is represented by $\lambda_{\min }$ and $\lambda_{\max }$. If the $\lambda$ range is set too narrow, the state trajectory and the resulting FE may get distorted. If the $\lambda$ range is set too wide, the simulation will take excessively long time to run.

In this section, the effects of the $\lambda$ range on fuel economy and $\mathrm{NO}_{\mathrm{x}}$ emissions of ECMSwDP algorithm are examined with the assumed $\lambda$ range varying from $\lambda_{\text {min }} \in$ $[0,1,2]$ to $\lambda_{\max }=10$. As shown in Figure 5.13 , the increase in the range of $\lambda$ by increasing the $\lambda_{\max }$ improves the $\mathrm{FE}$ for each $\lambda_{\min }$ examined. The operation of this 
vehicle with $\lambda$ range from $\lambda_{\min } \in[0,1]$ to $\lambda_{\max }=10$ was shown to significantly decrease the $\mathrm{NO}_{\mathrm{x}}$ emissions. The $\lambda$ range from $\lambda_{\min }=2$ to $\lambda_{\max }=10$, the reduction in $\mathrm{NO}_{\mathrm{x}}$ emissions is negligible.

The minimum value of $\lambda$ in the range $\left(\lambda_{\min }\right)$ apparently affected on the $\mathrm{NO}_{\mathrm{x}}$ reduction. The smaller $\lambda_{\min }$, the greater the value of reduction in $\mathrm{NO}_{\mathrm{x}}$ emissions. This algorithm prefers to use more battery power, which then decreased the $\mathrm{NO}_{\mathrm{x}}$ emissions. The wider $\lambda$ range and smaller $\lambda_{\min }$ provides more potential for the vehicle to achieve better fuel consumption and lower $\mathrm{NO}_{\mathrm{x}}$ emissions than the narrower $\lambda$ range (resolution) and higher $\lambda_{\text {min }}$, but requires more computational resources.

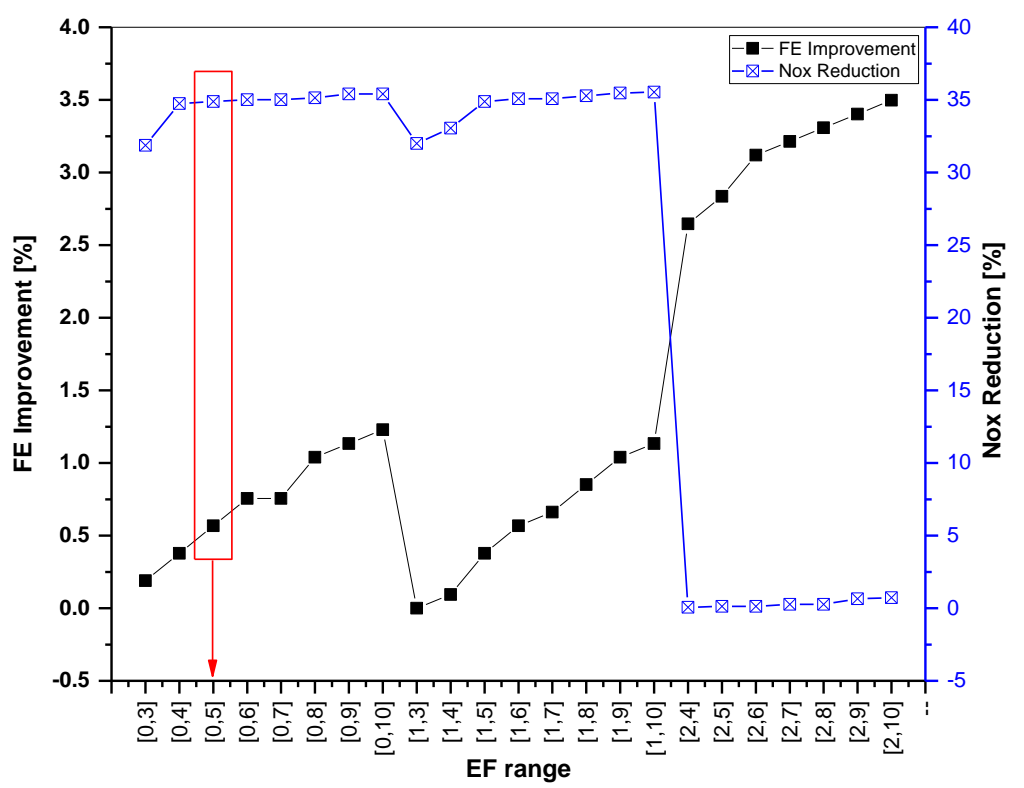

Figure 5.13 Effect of $\lambda$ range on the $\mathrm{FE}$ and $\mathrm{NO}_{\mathrm{x}}$ Emissions

For example, at each time step, if the $\lambda$ range $[0,10]$ is considered, there are 110 nodes ( $P \in[0: 0.1: 10])$, and $110 \times 2000$ combinations $(L \in[0.4: 0.0001: 0.6])$ involved, which is larger than the $60 \times 2000$ iterations when the $\lambda$ range $[0,5]$ is selected.

As shown in Figure 5.13, the $\lambda$ range $[0,5]$ achieves the best compromise of FE improvement, $\mathrm{NO}_{\mathrm{x}}$ reduction and the computational effort especially when the $\mathrm{NO}_{\mathrm{x}}$ emissions are a major concern. 


\section{The study case with $\lambda$ ranges of $[0,5]$ and $[2,4]$}

Table 5.5 compares the $\mathrm{FE}$ and $\mathrm{NO}_{\mathrm{x}}$ emissions simulated with $\lambda$ ranges of $[0,5]$ and $[2,4]$.

The FE with a $\lambda$ range of $[0,5]$ is $10.64 \mathrm{mpg}$, which is $2 \%$ lower than the $10.86 \mathrm{mpg}$ observed with a $\lambda$ range of $[2,4]$. The $\mathrm{NO}_{\mathrm{x}}$ emissions observed with a $\lambda$ range of $[0,5]$ is $9.95 \mathrm{gram} / \mathrm{mi}$, which is $34.88 \%$ lower than the $15.28 \mathrm{~g} / \mathrm{mile}$ observed with a $\lambda$ range of [ 2 , 4].

Table 5.5 Simulation results of different $\lambda$ range obtained by ECMSwDP over the Beijing cycle

\begin{tabular}{|c|c|c|c|}
\hline & FE $(\mathbf{m p g})$ & FC $($ gram/mi) & NOx $($ gram/mi) \\
\hline DP $\boldsymbol{\gamma}=\mathbf{1 5}, \boldsymbol{\lambda} \in[\mathbf{2 , 4}]$ & 10.86 & 290.09 & 15.28 \\
\hline DP $\boldsymbol{\gamma}=\mathbf{1 5}, \boldsymbol{\lambda} \in[\mathbf{0 , 5}]$ & 10.64 & 295.96 & 9.95 \\
\hline
\end{tabular}
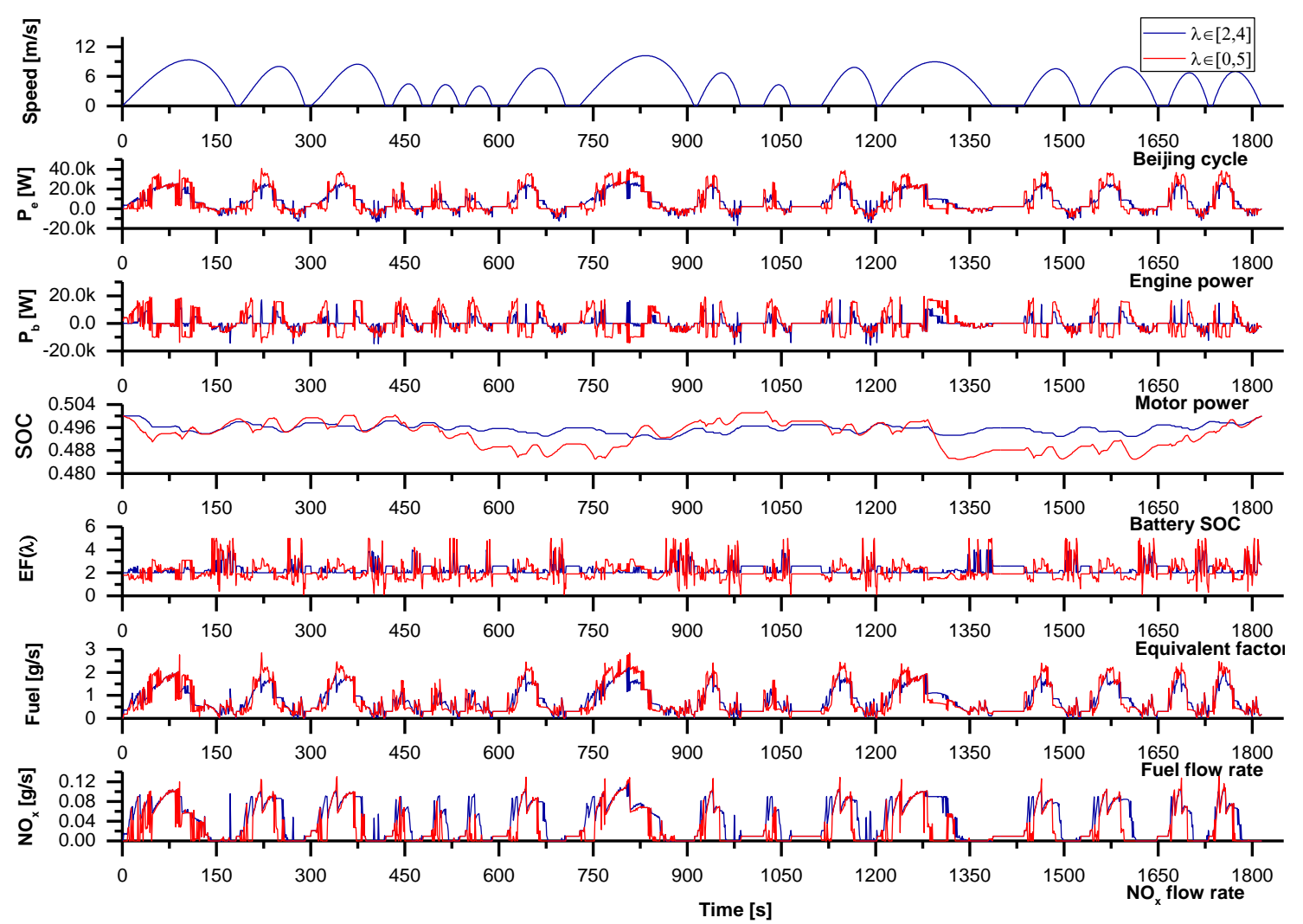

Figure 5.14 Comparison of the different $\lambda$ ranges obtained by ECMSwDP over the Beijing cycle 
Figure 5.14 compares the operation of this vehicle over the Beijing cycle simulated using ECMSwDP with $\lambda$ ranges of $[0,5]$ and $[2,4]$. The larger the range of $\lambda$, the more battery power is used, and more engine power is needed to propel the vehicle and charge the battery. An increased electrical motor only operation time achieved a large $\mathrm{NO}_{\mathrm{x}}$ emissions reduction and more FE improvement. However, the battery charging and discharging losses will future offset the FE improvement observed with a $\lambda$ range of $[0,5]$.

\subsection{Summary}

An ECMSwDP model was designed in a backward vehicle simulator for a parallel electric hybrid transit bus. The objective of the ECMSwDP algorithm is to find the globally optimal $\lambda$ for the application of ECMS in optimizing the operation of the hybrid vehicle. The fuel economy observed using ECMS with $\lambda$ derived using ECMSwDP is comparable to that observed using DP over the Beijing cycle. The simulation results obtained over the Beijing cycle and WVU-CSI cycle demonstrated that the average of the $\lambda$ extracted using ECMSwDP is comparable to the optimal one. In this research, the average of the $\lambda$ value extracted using ECMSwDP can be as the appropriate initial $\lambda$ value noted as $\lambda_{0}$ of the ECMS with penalty and the appropriate optimal $\lambda$ of ECMS without penalty. The fuel economy of this hybrid vehicle operated over the Beijing cycle obtained using ECMS with and without penalty function with the average $\lambda$ extracted using ECMSwDP is only $3 \%$ and $9 \%$ lower than those observed using DP.

The average extracted time variant $\lambda(t)$ from ECMSwDP can provide a new method for the selection of the initial $\lambda_{0}$ value of the ECMS algorithm with a penalty. The ECMSwDP algorithm is sensitive to the road grade and achieves performance improvement in fuel economy than rule-based control strategy, because of the enough buffer in the battery on the top of the hill in anticipation of future regeneration energy. Also, the $\lambda$ range influences 
the resolution of the control variable in the ECMSwDP algorithm, which plays an important role in determining the accuracy and performance of the ECMSwDP.

In conclusion, the ECMSwDP algorithm provides the foundation for the development of a robust, real-time and suboptimal control strategy of hybrid electric transit bus. 


\section{Driving Pattern Identification Model}

The determination of the appropriate $\lambda$ using ECMSwDP needs known bus driving patterns beforehand, which are usually not available prior to the departure of the vehicle. Accordingly, there is a need to develop a model capable of predicting the upcoming driving pattern. This is critical for the vehicle controller to determine the appropriate $\lambda$. The control strategy developed based on the predicted driving pattern can be more adaptive to a variety of routes and driving conditions. There are two main methods capable of predicting the driving patterns. The first one is to predict with the information reported by the GIS, GPS, ITS, V2V or V2I (Marano, et al. 2012). The second one is to predict the upcoming driving patterns through analyzing the historical driving information from an ECU data logger and current driving condition from a vehicle controller area network (He, Sun and Zhang 2012). In this thesis, the future driving patterns are predicted using the past and current driving conditions, followed by a short prediction scope including numerous distance segments, and concluding with a full route prediction.

\subsection{Driving Pattern Features}

The fuel economy and exhaust emissions are affected by the driving patterns. An analysis of HEBs operation cycles leads to the development of the numbers of features needed for the accurate description of the driving pattern. Montazeri-Gh, Fotouhi, \& Naderpour (2011) introduced a group of 21 features needed in describing the driving patterns. In this thesis, 15 features, showed in Table 6.1, have been examined. 
Table 6.1 The driving pattern features

\begin{tabular}{|c|c|c|c|c|}
\hline & Symbol & Explanation & Formula & Units \\
\hline$P 1$ & $\bar{v}$ & Average speed with idle & $\bar{v}=\frac{\int v d t}{t}$ & $\mathrm{mph}$ \\
\hline$P 2$ & $\bar{v}_{\text {no idle }}$ & $\begin{array}{c}\text { Average speed } \\
\text { without idle }\end{array}$ & $\bar{v}_{\text {no idle }}=\bar{v} \cdot \frac{1}{1-\text { Idle }(\%) / 100}$ & $\mathrm{mph}$ \\
\hline$P 3$ & $v_{\max }$ & Maximum speed & $v_{\max }=\max \left(v_{i}, i=1,2, \cdots, k\right)$ & $\mathrm{mph}$ \\
\hline$P 4$ & $\sigma_{v}$ & Standard deviation speed & $\sigma_{v}=\sqrt{\frac{1}{n_{v}-1} \sum_{i=1}^{N}\left(v_{i}-\bar{v}\right)^{2}}, v_{i}<0$ & $\mathrm{mph}$ \\
\hline P5 & $P_{i}$ & Idle time percent & $\begin{array}{c}P_{i}=\frac{t_{i}}{t} \\
t_{i} \text { is the idle time }\end{array}$ & $\%$ \\
\hline P6 & $\widehat{N}$ & Number of stops per mile & $\widehat{N}=\frac{N}{d}$ & - \\
\hline$P 7$ & $\bar{T}_{m i c}$ & Average micro trip time & $\begin{array}{c}\bar{T}_{m i c}=\frac{t}{n_{m i c}} \\
n_{m i c} \text { is the number of micro trip }\end{array}$ & $\mathrm{s}$ \\
\hline$P 8$ & $\bar{\alpha}_{+}$ & Average acceleration & $\begin{array}{c}\bar{\alpha}_{+}=\frac{1}{t_{+}} \sum_{i=1}^{N} \alpha_{i}, \alpha_{i}>0 \\
t_{+} \text {is the time with } \alpha_{i}>0\end{array}$ & $\mathrm{~g}$ \\
\hline$P 9$ & $\bar{\alpha}_{-}$ & Average deceleration & $\begin{array}{c}\bar{\alpha}_{-}=\frac{1}{t_{+}} \sum_{i=1}^{N} \alpha_{i}, \alpha_{i}<0 \\
t_{+} \text {is the time with } \alpha_{i}<0\end{array}$ & $\mathrm{~g}$ \\
\hline$P 10$ & $P_{a}$ & Acceleration time percent & $\begin{array}{c}P_{a}=\frac{t_{+}}{t} \\
t_{+} \text {is acceleration time }\end{array}$ & $\%$ \\
\hline$P 11$ & $P_{d}$ & Deceleration time percent & $\begin{array}{c}P_{a}=\frac{t_{d}}{t} \\
t_{d} \text { is deceleration time }\end{array}$ & $\%$ \\
\hline$P 12$ & $\sigma_{a}$ & Standard deviation acceleration & $\begin{array}{c}\sigma_{a}=\sqrt{\frac{1}{n_{a}-1} \sum_{i=1}^{N}\left(\alpha_{i}-\bar{\alpha}\right)^{2}}, \alpha_{i}>0 \\
n_{a} \text { is the number of acceleration }\end{array}$ & $\mathrm{g}$ \\
\hline$P 13$ & $\sigma_{d}$ & Standard deviation deceleration & $\begin{array}{c}\sigma_{d}=\sqrt{\frac{1}{n_{d}-1} \sum_{i=1}^{N}\left(\alpha_{i}-\bar{\alpha}\right)^{2}}, \alpha_{i}<0 \\
n_{d} \text { is the number of deceleration }\end{array}$ & $\mathrm{g}$ \\
\hline$P 14$ & $R M S_{a}$ & Root mean squared acceleration & $R M S_{a}=\sqrt{\frac{\sum_{i=1}^{N} \alpha_{i}^{2}}{n_{a}}}, \alpha_{i}>0$ & $\mathrm{~g}$ \\
\hline$P 15$ & $P K E^{*}$ & $\begin{array}{l}\text { Positive acceleration } \\
\text { kinetic energy }\end{array}$ & $\begin{array}{c}P K E=\frac{\sum\left(v_{f}^{2}-v_{i}^{2}\right)}{d}, \alpha>0 \\
v_{f}=\text { Final speed }(\mathrm{mpg}) v_{i}=\text { Initial speed }(\mathrm{mpg}) \\
d=\text { Section length }(\mathrm{mile})\end{array}$ & $\mathrm{g}$ \\
\hline
\end{tabular}

${ }^{*}$ The term PKE represents the sum of the positive kinetic energy changes during acceleration in $\mathrm{m} / \mathrm{s}^{2}$

\subsubsection{The Key Driving Pattern Features for the Description of the Driving Pattern}

The more number of driving features employed, the more accurate in describing the characteristics of the driving patterns involved. However, some features dependent of others and considered redundant, which can be numerically identified and excluded from 
the list of key driving features. In this research, the dominating driving features will be identified by eliminating the redundant features using the following methods.

\section{Principal component analysis (PCA)}

Table 6.2 shows the values of the 15 features of the available 54 test cycles. In this research, the number of vehicle driving features is reduced by principal component analysis (PCA), an independent method widely used to reduce data dimension, and eliminate multicollinearity (Jolliffe 2002). The set of features includes less variables but still provides the information necessary to accurately describe the main characteristics of the driving cycle. The following is the brief description of the PCA process (Smith 2002):

a. The normalization of the raw data: The indices are of different orders of magnitude, so normalization is needed. With the assumed characteristics of driving cycle of $x_{i}, i=$ $1,2, \cdots p$, each variable can be normalized as follows:

$$
\hat{x}_{i}=\frac{x_{i}-\bar{x}_{i}}{\sigma_{x_{i}}}
$$

Where $\bar{x}_{i}$, and $\sigma_{x_{i}}$ is the average and standard deviation of $x_{i}$, respectively.

b. The calculation of the covariance matrix $R$.

c. The calculation of the eigenvectors $\lambda_{1} \geq \lambda_{2} \cdots \geq \lambda_{q}$ and eigenvalues $U_{1}, U_{2} \cdots, U_{q}$ of the covariance matrix R.

d. The calculation of the contribution rate:

$$
E_{i}=\frac{\lambda_{i}}{\sum_{i=1}^{K} \lambda_{i}}
$$

e. The calculation of the $m$ principal components:

$$
C_{i}=\left(\hat{x}_{1}, \hat{x}_{2}, \cdots \hat{x}_{K}\right) E_{i}, \quad i=1,2, \cdots m \quad \text { Equation } 6.3
$$

Figure 6.1 shows the eigenvalues associated with a component. The eigenvalues of the first three principal components are greater than 1, and the eigenvalues of the remainder principal components are relatively small and less than 1 . The first three principal 
components will be used to conduct the regression analysis. From the calculation results, the first three principal components contribute $47.6 \%, 29.3 \%$, and $11.7 \%$ to the whole information of the original data set. In other words, up to $85.6 \%$ of the variances can be accurately described by the first three main components.

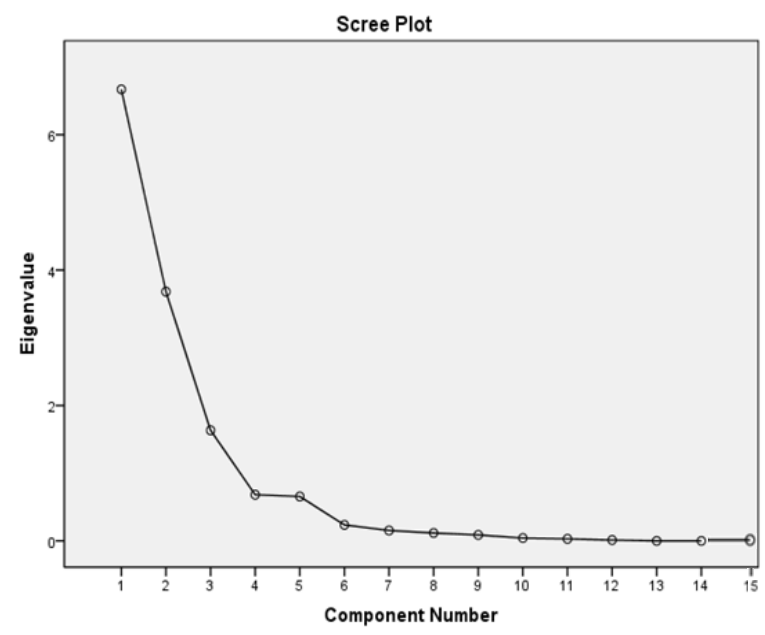

Figure 6.1 The eigenvalues associated with a component

The component matrix of PCA shows the loading of each variable on each principal component. As shown in Table 6.3, some pairs of variables $\left(\bar{a}_{+} \& \bar{a}_{-}\right.$and $\left.\bar{v} \& \bar{v}_{\text {no idle }}\right)$ were found to have approximately same load on the components. It is evident that these pairs are related to each other or dependents. Accordingly, the average speed without idle $\bar{v}_{\text {no idle }}$ and the average deceleration $\bar{a}_{-}$were excluded from the list of vehicle operation features. The number of vehicle operation features are decreased from 15 to 13 for the further regression analysis. 


\section{Table 6.2 The driving features of the current 54 heavy-duty drive cycles collected}

\begin{tabular}{|c|c|c|c|c|c|c|c|c|c|c|c|c|c|c|c|c|c|}
\hline Schedule & Description & $\begin{array}{c}\bar{v} \\
(\mathbf{m p h})\end{array}$ & $\begin{array}{l}\bar{v}_{\text {noidele }} \\
\text { (mph) }\end{array}$ & $\begin{array}{r}v_{\max } \\
(\mathrm{mph})\end{array}$ & $\underset{(\mathrm{mph})}{\sigma_{v}}$ & $\begin{array}{c}P_{i} \\
(\%)\end{array}$ & $\begin{array}{l}\widehat{N} \\
(-)\end{array}$ & $\begin{array}{c}\bar{T}_{\text {mic }} \\
(\mathrm{s})\end{array}$ & $\begin{array}{l}\bar{\alpha}_{+} \\
(\mathrm{g})\end{array}$ & $\begin{array}{l}\bar{\alpha}_{-} \\
(\mathrm{g})\end{array}$ & $\begin{array}{c}P_{a} \\
(\%)\end{array}$ & $\begin{array}{c}P_{d} \\
(\%)\end{array}$ & $\begin{array}{l}\sigma_{a} \\
(\mathrm{~g})\end{array}$ & $\begin{array}{l}\sigma_{d} \\
(\mathrm{~g})\end{array}$ & $\underset{(\mathrm{g})}{R M S_{a}}$ & $\begin{array}{c}P K E \\
(g)\end{array}$ & $\underset{(\%)}{H E R P I}$ \\
\hline Arterial & Arterial Segment of BAC & 23.2 & 29.6 & 40.0 & 16.3 & 21.9 & 2.0 & 60.8 & $\begin{array}{ll}2.3 \mathrm{E}- \\
02\end{array}$ & $\begin{array}{l}2.3 \mathrm{E}- \\
02\end{array}$ & 38.3 & 12.5 & \begin{tabular}{|l|}
$3.3 \mathrm{E}-$ \\
02
\end{tabular} & $\begin{array}{l}6.4 \mathrm{E}- \\
02\end{array}$ & $\begin{array}{l}\begin{array}{l}7.9 \mathrm{E}- \\
02\end{array} \\
02\end{array}$ & 0.4 & 52.7 \\
\hline BAC & Business Arterial Commuter & 17.8 & 23.8 & 55.0 & 16.2 & 25.1 & 3.6 & 41.6 & $\begin{array}{l}2.0 \mathrm{E}- \\
02\end{array}$ & $\begin{array}{l}2.0 \mathrm{E}- \\
02\end{array}$ & 27.8 & 10.6 & \begin{tabular}{|l|}
$3.5 \mathrm{E}-$ \\
02
\end{tabular} & $\begin{array}{l}5.8 \mathrm{E}- \\
02\end{array}$ & $\begin{array}{l}\begin{array}{l}7.4 \mathrm{E}- \\
02\end{array} \\
0\end{array}$ & 0.3 & 42.8 \\
\hline BEELINE & WCDOT Bus Cycle & 14.4 & 20.0 & 49.7 & 14.8 & 25.3 & 3.5 & 53.0 & $\begin{array}{l}2.3 \mathrm{E}- \\
02\end{array}$ & $\begin{array}{l}2.3 \mathrm{E}- \\
02\end{array}$ & 39.8 & 32.5 & $\begin{array}{l}3.8 \mathrm{E}- \\
02\end{array}$ & $\begin{array}{l}4.6 \mathrm{E}- \\
02\end{array}$ & $\begin{array}{l}6.8 \mathrm{E}- \\
02\end{array}$ & 0.8 & 58.4 \\
\hline Braunschweig & Braunschweig City Bus Cycle & 13.9 & 19.0 & 36.2 & 11.4 & 25.4 & 4.3 & 44.8 & $\begin{array}{l}2.2 \mathrm{E}- \\
02\end{array}$ & $\begin{array}{l}2.2 \mathrm{E}- \\
02 \\
\end{array}$ & 38.7 & 29.6 & \begin{tabular}{|l|}
$4.0 \mathrm{E}-$ \\
02
\end{tabular} & $\begin{array}{l}4.9 \mathrm{E}- \\
02\end{array}$ & $\begin{array}{l}7.1 \mathrm{E}- \\
02\end{array}$ & 0.6 & 55.0 \\
\hline CBD & Central Business District Cycle & 12.9 & 16.2 & 20.0 & 8.2 & 20.1 & 7.0 & 32.0 & $\begin{array}{l}2.3 \mathrm{E}- \\
02\end{array}$ & $\begin{array}{l}2.3 \mathrm{E}- \\
02\end{array}$ & 27.5 & 12.5 & $\begin{array}{l}3.9 \mathrm{E}- \\
02\end{array}$ & $\begin{array}{l}6.2 \mathrm{E}- \\
02\end{array}$ & $\begin{array}{ll}8.0 \mathrm{E}- \\
02\end{array}$ & 2.1 & 48.0 \\
\hline CBD_Truck & Central Business District Cycle Truck & 9.2 & 11.5 & 20.0 & 7.1 & 18.7 & 6.4 & 49.4 & $\begin{array}{l}1.5 \mathrm{E}- \\
02\end{array}$ & $\begin{array}{l}1.5 \mathrm{E}- \\
02\end{array}$ & 50.6 & 26.1 & \begin{tabular}{|l|l|}
$1.5 \mathrm{E}-$ \\
02
\end{tabular} & $\begin{array}{ll}2.6 \mathrm{E}- \\
02\end{array}$ & $\begin{array}{l}3.7 \mathrm{E}-\mathrm{-} \\
02\end{array}$ & 1.6 & 56.4 \\
\hline CILCC & Composite International Truck Local Cycle and Commuter & 13.9 & 16.9 & 55.0 & 12.4 & 17.6 & 2.0 & 105.2 & $\begin{array}{l}8.5 \mathrm{E}- \\
03\end{array}$ & $\begin{array}{l}8.5 \mathrm{E}- \\
03\end{array}$ & 15.4 & 13.5 & $\begin{array}{l}2.1 \mathrm{E}- \\
02\end{array}$ & $\begin{array}{l}2.2 \mathrm{E}- \\
02\end{array}$ & $\begin{array}{l}3.3 \mathrm{E}- \\
02\end{array}$ & 0.2 & 38.0 \\
\hline CSHVC & City Suburban Heavy-Duty Vehicle & 14.1 & 18.8 & 43.8 & 13.1 & 23.3 & 1.9 & 100.3 & $\begin{array}{l}1.5 \mathrm{E}- \\
02\end{array}$ & $\begin{array}{l}1.5 \mathrm{E}- \\
02\end{array}$ & 36.7 & 30.4 & \begin{tabular}{|l|}
$2.5 \mathrm{E}-$ \\
02
\end{tabular} & $\begin{array}{l}2.9 \mathrm{E}- \\
02\end{array}$ & $\begin{array}{l}4.4 \mathrm{E}- \\
02\end{array}$ & 0.4 & 51.7 \\
\hline CSHVR & Commuter segment of BAC & 14.1 & 18.8 & 43.8 & 13.1 & 23.3 & 1.9 & 100.3 & $\begin{array}{l}.5 \mathrm{E}- \\
02\end{array}$ & $\begin{array}{l}1.5 \mathrm{E}- \\
02\end{array}$ & 36.7 & 30.4 & $\begin{array}{l}2.5 \mathrm{E}- \\
02\end{array}$ & $\begin{array}{l}2.9 \mathrm{E}- \\
02\end{array}$ & $\begin{array}{l}4.4 \mathrm{E}- \\
02\end{array}$ & 0.4 & 51.7 \\
\hline Commuter-DieselNet & European Urban Drive Cycle & 46.3 & 49.7 & 55.0 & 16.8 & 6.8 & 0.2 & 290.0 & $\begin{array}{l}8.1 \mathrm{E}- \\
03\end{array}$ & $\begin{array}{l}8.1 \mathrm{E}- \\
03\end{array}$ & 28.0 & 4.2 & \begin{tabular}{|l|}
$1.9 \mathrm{E}-$ \\
02
\end{tabular} & $\begin{array}{l}3.9 \mathrm{E}- \\
02\end{array}$ & $\begin{array}{l}\begin{array}{l}4.5 \mathrm{E}- \\
02\end{array} \\
0\end{array}$ & 0.7 & 18.6 \\
\hline Commuter & $\begin{array}{l}\text { European Cycle for Emissions Certification of Heavy-duty } \\
\text { Diesel Vehicles }\end{array}$ & 42.3 & 49.7 & 55.0 & 20.7 & 14.7 & 0.2 & 291.0 & $\begin{array}{l}7.4 \mathrm{E}- \\
03\end{array}$ & $\begin{array}{l}7.4 \mathrm{E}- \\
03\end{array}$ & 27.0 & 4.1 & $\begin{array}{l}1.8 \mathrm{E}- \\
02\end{array}$ & $\begin{array}{l}3.7 \mathrm{E}- \\
02\end{array}$ & $\begin{array}{l}4.3 \mathrm{E}- \\
02\end{array}$ & 0.7 & 18.4 \\
\hline Commuter310 & Rural and Motorway Sections of ETC Cycle & 42.3 & 49.7 & 55.0 & 20.7 & 15.0 & 0.2 & 290.0 & $\begin{array}{l}7.4 \mathrm{E}- \\
03\end{array}$ & $\begin{array}{l}7.4 \mathrm{E}- \\
03\end{array}$ & 25.5 & 3.8 & \begin{tabular}{|l|}
$1.8 \mathrm{E}-$ \\
02
\end{tabular} & $\begin{array}{l}3.7 \mathrm{E}- \\
02\end{array}$ & $\begin{array}{l}4.3 \mathrm{E}- \\
02\end{array}$ & 0.7 & 18.6 \\
\hline Commuter No Init_Idle & European Transient Cycle, Segment1 & 46.3 & 49.7 & 55.0 & 16.8 & 6.8 & 0.2 & 290.0 & $\begin{array}{l}8.1 \mathrm{E}- \\
03\end{array}$ & $\begin{array}{l}8.1 \mathrm{E}- \\
03\end{array}$ & 28.0 & 4.2 & $\begin{array}{l}1.9 \mathrm{E}- \\
02 \\
\end{array}$ & $\begin{array}{l}3.9 \mathrm{E}- \\
02\end{array}$ & $\begin{array}{l}4.5 \mathrm{E}- \\
02 \\
\end{array}$ & 0.7 & 18.6 \\
\hline ECE_15 & Extra Urban Driving Cycle & 11.3 & 16.8 & 31.1 & 10.6 & 32.7 & 4.9 & 44.0 & $\begin{array}{l}1.4 \mathrm{E}- \\
02\end{array}$ & $\begin{array}{l}.4 \mathrm{E}- \\
02\end{array}$ & 21.4 & 18.4 & \begin{tabular}{|l|}
$2.8 \mathrm{E}-$ \\
02
\end{tabular} & $\begin{array}{l}3.0 \mathrm{E}- \\
02\end{array}$ & $\begin{array}{l}\begin{array}{l}4.6 \mathrm{E}- \\
02\end{array} \\
02\end{array}$ & 4.2 & 50.9 \\
\hline ETC_FIGE & EUDC for Low Power Cycle & 36.5 & 38.2 & 56.5 & 17.8 & 3.8 & 0.3 & 346.4 & $\begin{array}{l}9.3 \mathrm{E}- \\
03\end{array}$ & $\begin{array}{l}9.3 \mathrm{E}- \\
03-\end{array}$ & 43.6 & 38.2 & \begin{tabular}{|l|}
$1.8 \mathrm{E}-$ \\
02
\end{tabular} & $\begin{array}{l}2.9 \mathrm{E}- \\
02\end{array}$ & $\begin{array}{l}3.7 \mathrm{E}- \\
02\end{array}$ & 0.1 & 15.5 \\
\hline ETC_Rur_Mot & Freeway Operation of Heavy-duty Trucks & 45.8 & 47.8 & 56.5 & 12.3 & 4.2 & 0.1 & 1199.0 & $\begin{array}{l}6.7 \mathrm{E}- \\
03 \\
\end{array}$ & $\begin{array}{l}6.7 \mathrm{E}- \\
03 \\
\end{array}$ & 41.4 & 36.9 & \begin{tabular}{|l}
$1.6 \mathrm{E}-$ \\
02 \\
\end{tabular} & $\begin{array}{l}2.9 \mathrm{E}- \\
02\end{array}$ & $\begin{array}{l}.5 \mathrm{E}- \\
02 \\
\end{array}$ & 0.1 & 10.3 \\
\hline ETC_Urban & Heavy Heavy-Duty Diesel Truck-Creep & 14.2 & 16.2 & 30.9 & 8.5 & 11.3 & 1.7 & 133.3 & $\begin{array}{l}1.4 \mathrm{E}- \\
02\end{array}$ & $\begin{array}{l}.4 \mathrm{E}- \\
02\end{array}$ & 44.6 & 37.8 & \begin{tabular}{|l|}
$2.1 \mathrm{E}-$ \\
02
\end{tabular} & $\begin{array}{l}2.7 \mathrm{E}- \\
02\end{array}$ & $\begin{array}{l}3.9 \mathrm{E}- \\
02\end{array}$ & 0.5 & 47.9 \\
\hline EUDC & Heavy Heavy-Duty Diesel Truck- Cruise & 38.8 & 43.3 & 74.6 & 19.6 & 10.5 & 0.2 & 359.0 & $\begin{array}{l}9.9 \mathrm{E}- \\
03\end{array}$ & $\begin{array}{l}9.9 \mathrm{E}- \\
03\end{array}$ & 25.7 & 10.5 & \begin{tabular}{|l|}
$1.9 \mathrm{E}-$ \\
02
\end{tabular} & $\begin{array}{l}3.0 \mathrm{E}- \\
02\end{array}$ & $\begin{array}{l}3.8 \mathrm{E}- \\
02\end{array}$ & 0.4 & 29.8 \\
\hline EUDC_Low & Heavy Heavy-Duty Diesel Truck- Trans & 36.9 & 41.2 & 55.9 & 17.1 & 10.5 & 0.2 & 359.0 & $\begin{array}{l}7.8 \mathrm{E}- \\
03\end{array}$ & $\begin{array}{l}7.8 \mathrm{E}- \\
03\end{array}$ & 18.0 & 7.5 & $\begin{array}{l}1.8 \mathrm{E}- \\
02\end{array}$ & $\begin{array}{l}2.9 \mathrm{E}- \\
02\end{array}$ & $\begin{array}{l}3.6 \mathrm{E}- \\
02\end{array}$ & 0.3 & 24.7 \\
\hline Freeway & Arterial Segment of BAC & 34.1 & 38.3 & 60.7 & 21.8 & 10.5 & 0.3 & 293.8 & $\begin{array}{l}8.1 \mathrm{E}- \\
03 \\
\end{array}$ & $\begin{array}{l}8.1 \mathrm{E}- \\
03 \\
\end{array}$ & 38.8 & 37.4 & \begin{tabular}{|l}
$1.4 \mathrm{E}-$ \\
02 \\
\end{tabular} & $\begin{array}{l}1.7 \mathrm{E}- \\
02 \\
\end{array}$ & $\begin{array}{l}2.5 \mathrm{E}- \\
02 \\
\end{array}$ & 0.2 & 13.5 \\
\hline HHDDT_All & Business Arterial Commuter & 31.2 & 35.8 & 59.3 & 23.6 & 12.3 & 0.5 & 202.7 & $\begin{array}{l}6.8 \mathrm{E}- \\
03\end{array}$ & $\begin{array}{l}6.8 \mathrm{E}- \\
03-\end{array}$ & 42.1 & 37.9 & \begin{tabular}{|l|}
$1.5 \mathrm{E}-$ \\
02
\end{tabular} & $\begin{array}{l}1.8 \mathrm{E}- \\
02\end{array}$ & $\begin{array}{l}2.5 \mathrm{E}- \\
02-\end{array}$ & 0.1 & 10.6 \\
\hline HHDDT_Creep & WCDOT Bus Cycle & 1.8 & 3.1 & 8.2 & 2.0 & 41.3 & 24.1 & 49.7 & $\begin{array}{l}3.4 \mathrm{E}- \\
03\end{array}$ & $\begin{array}{l}3.4 \mathrm{E}- \\
03\end{array}$ & 18.1 & 26.8 & \begin{tabular}{|l|}
$1.3 \mathrm{E}-$ \\
02
\end{tabular} & $\begin{array}{l}1.2 \mathrm{E}- \\
02\end{array}$ & $\begin{array}{l}1.9 \mathrm{E}- \\
02\end{array}$ & 20.7 & 25.3 \\
\hline HHDDT_Cruise & Braunschweig City Bus Cycle & 39.9 & 43.4 & 59.3 & 22.0 & 7.8 & 0.3 & 320.3 & $\begin{array}{l}5.1 \mathrm{E}- \\
03\end{array}$ & $\begin{array}{l}5.1 \mathrm{E}- \\
03\end{array}$ & 43.8 & 40.3 & $\begin{array}{l}1.1 \mathrm{E}- \\
02\end{array}$ & $\begin{array}{l}1.2 \mathrm{E}- \\
02\end{array}$ & $\begin{array}{l}1.8 \mathrm{E}- \\
02\end{array}$ & 0.1 & 4.6 \\
\hline HHDDT_Trans & Central Business District Cycle & 15.3 & 18.4 & 47.5 & 13.4 & 16.4 & 1.8 & 111.8 & $\begin{array}{l}1.4 \mathrm{E}- \\
02 \\
\end{array}$ & $\begin{array}{l}1.4 \mathrm{E}- \\
02 \\
\end{array}$ & 42.8 & 32.0 & $\begin{array}{l}2.3 \mathrm{E}- \\
02 \\
\end{array}$ & $\begin{array}{l}2.9 \mathrm{E}- \\
02\end{array}$ & $\begin{array}{l}4.2 \mathrm{E}- \\
02 \\
\end{array}$ & 1.1 & 48.7 \\
\hline HWFET & EPA Highway Fuel Economy & 48.2 & 48.6 & 59.9 & 10.2 & 0.8 & 0.1 & 760.0 & $\begin{array}{l}8.7 \mathrm{E}- \\
03\end{array}$ & $\begin{array}{l}8.7 \mathrm{E}- \\
03\end{array}$ & 44.1 & 38.8 & \begin{tabular}{|l|}
$1.8 \mathrm{E}-$ \\
02
\end{tabular} & $\begin{array}{l}2.1 \mathrm{E}- \\
02\end{array}$ & $\begin{array}{l}3.0 \mathrm{E}- \\
02\end{array}$ & 0.3 & 15.9 \\
\hline Houston Bus Cycle (333) & $\begin{array}{l}\text { Metropolitan Transit Authority of Harris County, Houston, } \\
\text { TX }\end{array}$ & 11.1 & 18.5 & 31.8 & 11.7 & 39.4 & 6.0 & 33.1 & $\begin{array}{ll}1.5 \mathrm{E}- \\
02\end{array}$ & $\begin{array}{l}1.5 \mathrm{E}- \\
02\end{array}$ & 32.9 & 29.4 & \begin{tabular}{|l|}
$3.3 \mathrm{E}-$ \\
02
\end{tabular} & $\begin{array}{ll}3.6 \mathrm{E}- \\
02\end{array}$ & $\begin{array}{l}5.3 \mathrm{E}- \\
02\end{array}$ & 0.5 & 49.4 \\
\hline
\end{tabular}




\begin{tabular}{|c|c|c|c|c|c|c|c|c|c|c|c|c|c|c|c|c|c|}
\hline Int_Local & $\begin{array}{l}\text { City-suburban Drive Cycle, International Truck \& Engine } \\
\text { Corporation }\end{array}$ & 12.2 & 15.0 & 35.0 & 9.8 & 18.7 & 2.3 & 102.5 & $\begin{array}{l}8.1 \mathrm{E}- \\
03\end{array}$ & $\begin{array}{l}8.1 \mathrm{E}- \\
03\end{array}$ & 13.6 & 13.5 & $\begin{array}{l}2.1 \mathrm{E}- \\
02\end{array}$ & $\begin{array}{l}2.1 \mathrm{E}- \\
02\end{array}$ & $\begin{array}{l}3.2 \mathrm{E}- \\
02\end{array}$ & 0.9 & 40.2 \\
\hline $\mathrm{JE} 05$ & Japanese 2005 Heavy-duty Vehicle Cycle & 17.0 & 22.7 & 54.4 & 15.9 & 25.2 & 1.6 & 97.8 & $\begin{array}{l}1.2 \mathrm{E}- \\
02\end{array}$ & $\begin{array}{l}1.2 \mathrm{E}- \\
02\end{array}$ & 38.5 & 36.9 & $\begin{array}{l}2.4 \mathrm{E}- \\
02\end{array}$ & $\begin{array}{l}2.6 \mathrm{E}- \\
02\end{array}$ & $\begin{array}{l}4.0 \mathrm{E}- \\
02\end{array}$ & 0.3 & 37.7 \\
\hline KCM & King County Metro Bus Cycle & 23.4 & 28.9 & 60.0 & 18.1 & 18.9 & 1.9 & 66.4 & $\begin{array}{l}2.1 \mathrm{E}- \\
02\end{array}$ & $\begin{array}{l}2.1 \mathrm{E}- \\
02\end{array}$ & 42.2 & 29.5 & $\begin{array}{l}3.6 \mathrm{E}- \\
02\end{array}$ & $\begin{array}{l}4.9 \mathrm{E}- \\
02\end{array}$ & $\begin{array}{l}6.7 \mathrm{E}- \\
02\end{array}$ & 0.3 & 44.6 \\
\hline KCM1 & Section 1of KCM Cycle, $1-5$ & 41.8 & 46.5 & 60.0 & 18.9 & 10.2 & 0.2 & 450.0 & $\begin{array}{l}6.9 \mathrm{E}- \\
03 \\
\end{array}$ & $\begin{array}{l}6.9 \mathrm{E}- \\
03\end{array}$ & 23.2 & 26.9 & $\begin{array}{l}1.5 \mathrm{E}- \\
02\end{array}$ & $\begin{array}{l}1.6 \mathrm{E}- \\
02 \\
\end{array}$ & $\begin{array}{l}2.4 \mathrm{E}- \\
02 \\
\end{array}$ & 0.7 & 14.8 \\
\hline КСM2 & Section 2 of KCM Cycle, Rte-174 & 19.6 & 23.9 & 46.7 & 13.4 & 18.2 & 2.8 & 54.0 & $\begin{array}{l}2.8 \mathrm{E}- \\
02\end{array}$ & $\begin{array}{l}2.8 \mathrm{E}- \\
02\end{array}$ & 54.1 & 29.2 & $\begin{array}{l}3.7 \mathrm{E}- \\
02\end{array}$ & $\begin{array}{l}5.7 \mathrm{E}- \\
02\end{array}$ & $\begin{array}{l}7.8 \mathrm{E}- \\
02\end{array}$ & 1.7 & 59.7 \\
\hline КСM3 & Section 3 of KCM Cycle, Rte-120 & 16.4 & 21.3 & 38.3 & 12.8 & 23.1 & 3.6 & 46.5 & $\begin{array}{l}2.6 \mathrm{E}- \\
02\end{array}$ & $\begin{array}{l}2.6 \mathrm{E}- \\
02\end{array}$ & 48.2 & 29.2 & $\begin{array}{l}4.1 \mathrm{E}- \\
02\end{array}$ & $\begin{array}{l}5.7 \mathrm{E}- \\
02\end{array}$ & $\begin{array}{l}7.9 \mathrm{E}- \\
02\end{array}$ & 1.1 & 58.5 \\
\hline KCM4 & Section 4 of KCM Cycle, Rte-106 & 15.0 & 20.1 & 31.2 & 10.8 & 25.4 & 3.4 & 52.0 & $\begin{array}{l}2.3 \mathrm{E}- \\
02\end{array}$ & $\begin{array}{l}2.3 \mathrm{E}- \\
02\end{array}$ & 40.9 & 35.1 & $\begin{array}{l}4.0 \mathrm{E}- \\
02\end{array}$ & $\begin{array}{l}4.3 \mathrm{E}- \\
02\end{array}$ & $\begin{array}{l}6.7 \mathrm{E}- \\
02\end{array}$ & 3.1 & 57.9 \\
\hline Liberty & WCDOT Bus Cycle & 12.0 & 18.6 & 49.7 & 13.8 & 32.2 & 3.8 & 53.2 & $\begin{array}{l}1.9 \mathrm{E}- \\
02\end{array}$ & $\begin{array}{l}1.9 \mathrm{E}- \\
02\end{array}$ & 36.0 & 29.6 & $\begin{array}{l}3.4 \mathrm{E}- \\
02\end{array}$ & $\begin{array}{l}.1 \mathrm{E}- \\
02 \\
\end{array}$ & $\begin{array}{l}6.0 \mathrm{E}- \\
02\end{array}$ & 0.6 & 57.1 \\
\hline MCS & Mexico City Bus Schedule & 11.1 & 16.2 & 42.7 & 12.0 & 30.7 & 4.1 & 54.7 & $\begin{array}{l}1.7 \mathrm{E}- \\
02\end{array}$ & $\begin{array}{l}1.7 \mathrm{E}- \\
02\end{array}$ & 36.6 & 34.0 & $\begin{array}{l}3.3 \mathrm{E}- \\
02\end{array}$ & $\begin{array}{l}4.0 \mathrm{E}- \\
02\end{array}$ & $\begin{array}{l}5.7 \mathrm{E}- \\
02\end{array}$ & 0.3 & 53.8 \\
\hline Manhattan & Low Speed Bus Operation in Manhattan & 6.8 & 11.0 & 25.3 & 7.3 & 36.1 & 9.7 & 34.8 & $\begin{array}{l}2.0 \mathrm{E}- \\
02\end{array}$ & $\begin{array}{l}2.0 \mathrm{E}- \\
02\end{array}$ & 35.4 & 28.6 & $\begin{array}{l}3.6 \mathrm{E}- \\
02\end{array}$ & $\begin{array}{l}4.2 \mathrm{E}- \\
02\end{array}$ & $\begin{array}{l}.2 \mathrm{E}- \\
02\end{array}$ & 1.7 & 60.1 \\
\hline NYC BUS & Very Low Speed Bus Operation in New York City & 3.7 & 11.2 & 30.7 & 6.5 & 65.4 & 17.9 & 18.9 & $\begin{array}{l}1.5 \mathrm{E}- \\
02\end{array}$ & $\begin{array}{l}1.5 \mathrm{E}- \\
02\end{array}$ & 12.8 & 23.0 & $\begin{array}{l}4.8 \mathrm{E}- \\
02\end{array}$ & $\begin{array}{l}3.8 \mathrm{E}- \\
02\end{array}$ & $\begin{array}{l}6.4 \mathrm{E}- \\
02-\end{array}$ & 5.3 & 57.7 \\
\hline NY_Comp & Heavy-duty Vehicle, New York City & 8.8 & 13.3 & 36.0 & 9.5 & 33.2 & 7.6 & 36.2 & $\begin{array}{l}1.6 \mathrm{E}- \\
02\end{array}$ & $\begin{array}{l}1.6 \mathrm{E}- \\
02\end{array}$ & 33.2 & 29.1 & $\begin{array}{l}3.3 \mathrm{E}- \\
02\end{array}$ & $\begin{array}{l}3.5 \mathrm{E}- \\
02\end{array}$ & $\begin{array}{l}5.3 \mathrm{E}- \\
02 \\
\end{array}$ & 1.1 & 59.2 \\
\hline Nuremberg_R36 & Nuremberg Bus Route 36 & 8.9 & 12.8 & 33.2 & 8.9 & 30.8 & 9.0 & 31.2 & $\begin{array}{l}2.0 \mathrm{E}- \\
02\end{array}$ & $\begin{array}{l}2.0 \mathrm{E}- \\
02\end{array}$ & 34.8 & 36.5 & $\begin{array}{l}3.9 \mathrm{E}- \\
02\end{array}$ & $\begin{array}{l}3.7 \mathrm{E}- \\
02\end{array}$ & $\begin{array}{l}6.1 \mathrm{E}- \\
02\end{array}$ & 1.3 & 58.0 \\
\hline ОСТА & Orange County Transit Authority Bus Cycle & 12.3 & 16.2 & 40.6 & 10.3 & 21.3 & 4.7 & 48.5 & $\begin{array}{ll}2.1 \mathrm{E}- \\
02\end{array}$ & $\begin{array}{l}2.1 \mathrm{E}- \\
02\end{array}$ & 46.5 & 33.1 & $\begin{array}{l}3.6 \mathrm{E}- \\
02\end{array}$ & $\begin{array}{l}4.5 \mathrm{E}- \\
02\end{array}$ & $\begin{array}{l}6.5 \mathrm{E}- \\
02\end{array}$ & 0.7 & 56.3 \\
\hline Paris & ADEME-RATP Paris Bus Cycle & 6.6 & 10.3 & 29.7 & 7.3 & 34.7 & 13.4 & 26.6 & $\begin{array}{l}1.8 \mathrm{E}- \\
02\end{array}$ & $\begin{array}{l}1.8 \mathrm{E}- \\
02\end{array}$ & 34.3 & 32.2 & $\begin{array}{l}3.3 \mathrm{E}- \\
02\end{array}$ & $\begin{array}{l}4.0 \mathrm{E}- \\
02\end{array}$ & $\begin{array}{l}5.8 \mathrm{E}- \\
02-\end{array}$ & 0.9 & 57.4 \\
\hline ROUTE77 & Bus Route to BOS Airport, MA & 16.8 & 19.9 & 50.0 & 13.6 & 14.3 & 3.7 & 49.2 & $\begin{array}{l}2.0 \mathrm{E}- \\
02 \\
\end{array}$ & $\begin{array}{l}2.0 \mathrm{E}- \\
02 \\
\end{array}$ & 40.9 & 30.0 & $\begin{array}{l}3.8 \mathrm{E}- \\
02 \\
\end{array}$ & $\begin{array}{l}4.7 \mathrm{E}- \\
02 \\
\end{array}$ & $\begin{array}{l}6.7 \mathrm{E}- \\
02 \\
\end{array}$ & 1.0 & 47.1 \\
\hline SORT_1_Estimated & European Geometric Route for Urban Traffic & 7.5 & 12.5 & 24.9 & 8.0 & 39.4 & 9.3 & 31.3 & $\begin{array}{l}1.6 \mathrm{E}- \\
02\end{array}$ & $\begin{array}{l}1.6 \mathrm{E}- \\
02\end{array}$ & 23.2 & 21.9 & $\begin{array}{l}3.1 \mathrm{E}- \\
02\end{array}$ & $\begin{array}{l}3.2 \mathrm{E}- \\
02\end{array}$ & $\begin{array}{l}5.0 \mathrm{E}- \\
02\end{array}$ & 11.9 & 59.1 \\
\hline SORT_2_Estimated & European Geometric Route for Mixed Traffic & 11.1 & 16.9 & 31.1 & 11.3 & 33.5 & 5.2 & 41.0 & $\begin{array}{l}1.7 \mathrm{E}- \\
02\end{array}$ & $\begin{array}{l}1.7 \mathrm{E}- \\
02\end{array}$ & 27.6 & 22.2 & $\begin{array}{l}2.9 \mathrm{E}- \\
02\end{array}$ & $\begin{array}{l}3.3 \mathrm{E}- \\
02\end{array}$ & $\begin{array}{l}5.0 \mathrm{E}- \\
02\end{array}$ & 6.6 & 56.4 \\
\hline SORT_3_Estimated & European Geometric Route for Suburban Traffic & 15.7 & 19.5 & 37.3 & 12.5 & 19.8 & 3.3 & 55.3 & $\begin{array}{l}1.9 \mathrm{E}- \\
02\end{array}$ & $\begin{array}{l}1.9 \mathrm{E}- \\
02\end{array}$ & 35.3 & 24.6 & $\begin{array}{l}2.7 \mathrm{E}- \\
02\end{array}$ & $\begin{array}{l}3.4 \mathrm{E}- \\
02\end{array}$ & $\begin{array}{l}5.1 \mathrm{E}- \\
02\end{array}$ & 4.4 & 56.2 \\
\hline UDDS & EPA Heavy-duty Urban Dynamometer Driving Schedule & 18.8 & 28.5 & 58.0 & 19.8 & 33.3 & 2.5 & 50.6 & $\begin{array}{l}1.3 \mathrm{E}- \\
02\end{array}$ & $\begin{array}{l}.3 \mathrm{E}- \\
02\end{array}$ & 27.0 & 22.6 & $\begin{array}{l}3.0 \mathrm{E}- \\
02\end{array}$ & $\begin{array}{l}3.2 \mathrm{E}- \\
02 \\
\end{array}$ & $\begin{array}{l}4.8 \mathrm{E}- \\
02 \\
\end{array}$ & 0.6 & 39.9 \\
\hline VC_274 & Washington Metropolitan Area Transit Authority Bus Cycle & 21.1 & 30.6 & 58.0 & 21.6 & 29.0 & 2.7 & 45.4 & $\begin{array}{l}1.6 \mathrm{E}- \\
02\end{array}$ & $\begin{array}{l}1.6 \mathrm{E}- \\
02\end{array}$ & 39.5 & 32.8 & $\begin{array}{l}3.0 \mathrm{E}- \\
02\end{array}$ & $\begin{array}{l}3.8 \mathrm{E}- \\
02\end{array}$ & $\begin{array}{l}5.4 \mathrm{E}- \\
02 \\
\end{array}$ & 0.8 & 38.9 \\
\hline VC_28 & 5 Speed Truck Cycle & 7.3 & 12.9 & 36.5 & 9.4 & 39.5 & 8.5 & 35.1 & $\begin{array}{l}1.7 \mathrm{E}- \\
02\end{array}$ & $\begin{array}{l}1.7 \mathrm{E}- \\
02\end{array}$ & 33.6 & 28.1 & $\begin{array}{l}3.3 \mathrm{E}- \\
02\end{array}$ & $\begin{array}{l}3.8 \mathrm{E}- \\
02\end{array}$ & $\begin{array}{l}5.6 \mathrm{E}- \\
02\end{array}$ & 1.8 & 61.7 \\
\hline VC_637 & City Driving Cycle for Heavy Trucks & 27.4 & 31.4 & 58.0 & 19.3 & 11.9 & 0.9 & 123.5 & $\begin{array}{l}1.5 \mathrm{E}- \\
02\end{array}$ & $\begin{array}{l}1.5 \mathrm{E}- \\
02\end{array}$ & 45.2 & 36.2 & $\begin{array}{l}2.6 \mathrm{E}- \\
02\end{array}$ & $\begin{array}{l}3.9 \mathrm{E}- \\
02\end{array}$ & $\begin{array}{l}5.2 \mathrm{E}- \\
02-\end{array}$ & 0.4 & 33.5 \\
\hline WMATA & Yard Operation of Heavy-duty Trucks & 8.3 & 14.0 & 47.5 & 10.3 & 38.4 & 6.1 & 43.6 & $\begin{array}{l}1.5 \mathrm{E}- \\
02\end{array}$ & $\begin{array}{l}1.5 \mathrm{E}- \\
02\end{array}$ & 32.7 & 28.2 & $\begin{array}{l}2.8 \mathrm{E}- \\
02\end{array}$ & $\begin{array}{l}3.5 \mathrm{E}- \\
02 \\
\end{array}$ & $\begin{array}{l}5.0 \mathrm{E}- \\
02 \\
\end{array}$ & 0.9 & 58.9 \\
\hline WMATA_COMMUTER_2 & EPA Highway Fuel Economy & 34.6 & 53.3 & 67.0 & 27.8 & 34.9 & 0.1 & 526.0 & $\begin{array}{l}7.4 \mathrm{E}- \\
03\end{array}$ & $\begin{array}{l}\text { 7.4E- } \\
03\end{array}$ & 35.5 & 29.7 & $\begin{array}{l}1.7 \mathrm{E}- \\
02\end{array}$ & $\begin{array}{l}2.2 \mathrm{E}- \\
02\end{array}$ & $\begin{array}{l}3.0 \mathrm{E}- \\
02 \\
\end{array}$ & 0.5 & 16.9 \\
\hline WVU_5_Peak & $\begin{array}{l}\text { Metropolitan Transit Authority of Harris County, Houston, } \\
\text { TX }\end{array}$ & 20.0 & 24.4 & 39.9 & 13.5 & 17.9 & 1.0 & 148.0 & $\begin{array}{l}7.6 \mathrm{E}- \\
03\end{array}$ & $\begin{array}{l}7.6 \mathrm{E}- \\
03\end{array}$ & 23.4 & 13.0 & $\begin{array}{l}1.4 \mathrm{E}- \\
02\end{array}$ & $2.0 \mathrm{E}-$ & $2.7 \mathrm{E}-$ & 0.7 & 29.5 \\
\hline WVU_City & $\begin{array}{l}\text { City-suburban Drive Cycle, International Truck \& Engine } \\
\text { Corporation }\end{array}$ & 8.5 & 12.8 & 35.8 & 10.2 & 30.5 & 3.8 & 76.5 & $\begin{array}{l}1.1 \mathrm{E}- \\
02\end{array}$ & $\begin{array}{l}1.1 \mathrm{E}- \\
02\end{array}$ & 34.7 & 28.0 & $\begin{array}{l}2.0 \mathrm{E}- \\
02\end{array}$ & $\begin{array}{l}2.4 \mathrm{E}- \\
02\end{array}$ & $\begin{array}{l}3.5 \mathrm{E}- \\
02\end{array}$ & 1.1 & 51.5 \\
\hline Yard & Japanese 2005 Heavy-duty Vehicle Cycle & 3.3 & 6.5 & 16.8 & 4.7 & 47.2 & 12.0 & 47.3 & $\begin{array}{l}7.0 \mathrm{E}- \\
03\end{array}$ & $\begin{array}{l}7.0 \mathrm{E}- \\
03\end{array}$ & 24.8 & 19.1 & $\begin{array}{l}1.6 \mathrm{E}- \\
02\end{array}$ & $\begin{array}{l}1.9 \mathrm{E}- \\
02\end{array}$ & $\begin{array}{l}2.7 \mathrm{E}- \\
02\end{array}$ & 2.7 & 46.1 \\
\hline
\end{tabular}


Table 6.3 Component matrix of PCA

\begin{tabular}{|c|c|c|c|c|}
\hline & \multirow{2}{*}{ Variables } & \multicolumn{3}{|c|}{ Principal Component } \\
\hline & & 1 & 2 & 3 \\
\hline$P 1$ & $\bar{v}$ & -0.75 & 0.56 & -0.15 \\
\hline$P 2$ & $\bar{v}_{\text {noidle }}$ & -0.73 & 0.55 & -0.15 \\
\hline$P 3$ & $v_{\max }$ & 0.65 & -0.59 & -0.12 \\
\hline$P 4$ & $\sigma_{v}$ & -0.52 & 0.65 & -0.31 \\
\hline$P 5$ & $\boldsymbol{P}_{i}$ & 0.65 & -0.59 & -0.12 \\
\hline P6 & $\widehat{N}$ & 0.42 & -0.73 & 0.10 \\
\hline$P 7$ & $\overline{\boldsymbol{T}}_{\text {mic }}$ & -0.72 & 0.23 & 0.19 \\
\hline$P 8$ & $\bar{\alpha}_{+}$ & 0.85 & 0.50 & 0.02 \\
\hline$P 9$ & $\bar{\alpha}_{-}$ & 0.85 & 0.50 & 0.02 \\
\hline$P 10$ & $\boldsymbol{P}_{a}$ & 0.32 & 0.64 & 0.91 \\
\hline$P 11$ & $P_{d}$ & 0.29 & 0.20 & 0.68 \\
\hline$P 12$ & $\sigma_{a}$ & 0.79 & 0.29 & -0.13 \\
\hline$P 13$ & $\frac{u}{\sigma_{d}}$ & 0.73 & 0.57 & -0.25 \\
\hline$P 14$ & $\boldsymbol{R M S}_{a}$ & 0.64 & 0.49 & -0.18 \\
\hline P15 & PKE & 0.21 & 0.03 & 0.08 \\
\hline
\end{tabular}

Stepwise regression

The recovery of brake energy for the production of electricity was recognized as one of the major contributors to the improvement in fuel economy of a hybrid bus. As reported by Ehsani, Gao, \& Butler (1999), about $30 \%$ to $50 \%$ of the propulsion energy was converted to thermal energy during the braking process when operated in the urban driving condition. Transit buses usually operate at low average speed, acceleration/deceleration driving pattern. There is more potential brake energy to be recovered from transit buses than other vehicles which operate on relatively constant speed with less frequent deceleration and braking processes. The potential of the brake recovery energy is a critical characteristic in evaluating the potential benefit of the hybridization of a transit hybrid bus. Although the energy recovered is affected by the capacity and SOC of the battery, the maximum power of the generator, the maximum recoverable kinetic energy primarily depends on driving characteristics (Vyas and Bertram 2013).

In this research, Dr. Li proposes a new parameter noted as the hybrid energy recovery potential index (HERPI) defined as the ratio of the maximum electrical energy recoverable during the braking processes relative to the total propulsion energy needed in each specific cycle. It can be expressed as 


$$
H E R P I=\frac{E_{B R}}{E_{\text {trac }}}=\frac{\sum\left|\dot{E}_{\text {road }, i<0}\right| \cdot \Delta t_{i}}{\sum \dot{E}_{\text {road }, i>0} \cdot \Delta t_{i}}
$$

Where $E_{B R}$ is the potential braking energy, $E_{t r a c}$ is the total tractive energy, $\dot{E}_{\text {road }, i<0}$ is the braking power, $\dot{E}_{\text {road, } i>0}$ is the tractive power.

In this research, the HERPI of the 54 driving cycles was calculated and presented in Table 6.2. The HERPI will be considered as the response variable of stepwise regression in identifying the key driving pattern features dominating the HERPI. The flow chart for extracting the key driving pattern features is shown in Figure 6.2.

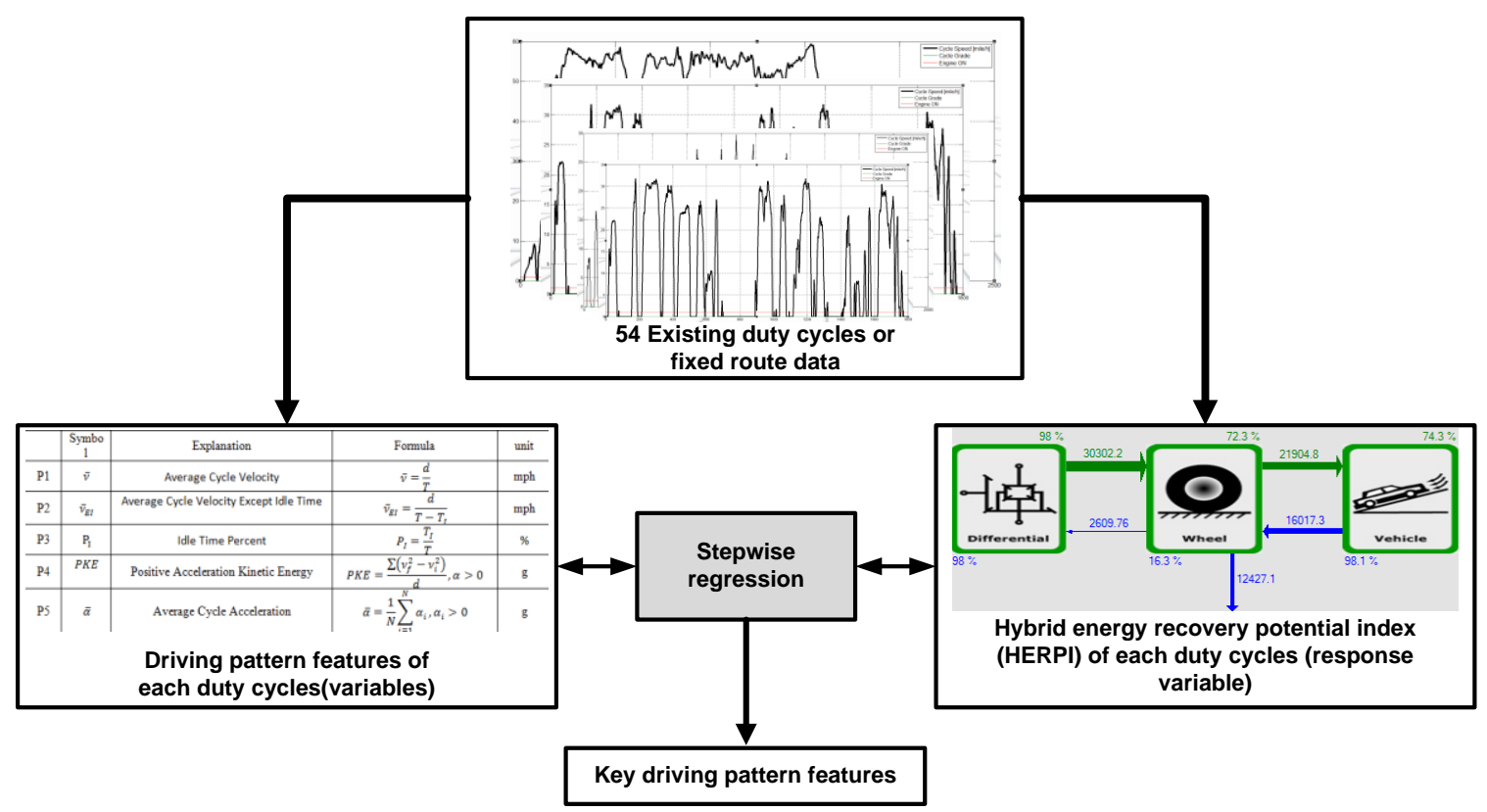

Figure 6.2 Flow chart of extracting key driving pattern features

The key driving pattern features having significant impact on the proposed HERPI were identified using the stepwise regression method. The output of the stepwise regression method is the minimum number of variables you would need to measure or predict the criterion variable (Hinman 2006). Stepwise regression was conducted using the software SPSS for Windows version 19. The regression results are shown in Table 6.4. 
Table 6.4 Stepwise regression coefficients

\begin{tabular}{|c|c|c|c|c|c|c|c|c|}
\hline \multirow[t]{2}{*}{ Model } & \multirow[t]{2}{*}{ R Square } & \multirow{2}{*}{\multicolumn{2}{|c|}{ Features and Symbol }} & \multicolumn{2}{|c|}{$\begin{array}{l}\text { Unstandardized } \\
\text { Coefficients }\end{array}$} & \multirow{2}{*}{$\begin{array}{c}\text { Standardized } \\
\text { Coefficients } \\
\text { Beta }\end{array}$} & \multirow[t]{2}{*}{$\mathrm{t}$} & \multirow[t]{2}{*}{ Sig. } \\
\hline & & & & B & Std. Error & & & \\
\hline \multirow[b]{2}{*}{1} & \multirow{2}{*}{0.587} & \multicolumn{2}{|l|}{ (Constant) } & 0.155 & 0.024 & & 6.392 & 0.000 \\
\hline & & $\begin{array}{c}\text { Average } \\
\text { acceleration }\end{array}$ & $\bar{\alpha}_{+}$ & 2.069 & 0.168 & 0.766 & 12.325 & 0.000 \\
\hline \multirow{3}{*}{2} & \multirow{3}{*}{0.859} & \multicolumn{2}{|l|}{ (Constant) } & 0.302 & 0.018 & & 17.231 & 0.000 \\
\hline & & $\begin{array}{c}\text { Average } \\
\text { acceleration }\end{array}$ & $\bar{\alpha}_{+}$ & 1.792 & 0.100 & 0.663 & 17.869 & 0.000 \\
\hline & & Average Speed & $\bar{v}$ & -0.016 & 0.001 & -0.532 & -14.332 & 0.000 \\
\hline \multirow{4}{*}{3} & \multirow{4}{*}{0.880} & \multicolumn{2}{|l|}{ (Constant) } & 0.376 & 0.024 & & 15.684 & 0.000 \\
\hline & & $\begin{array}{c}\text { Average } \\
\text { acceleration }\end{array}$ & $\bar{\alpha}_{+}$ & 1.937 & 0.099 & 0.717 & 19.482 & 0.000 \\
\hline & & Average Speed & $\bar{v}$ & -0.015 & 0.001 & -0.502 & -14.221 & 0.000 \\
\hline & & $\begin{array}{c}\text { Acceleration Time } \\
\text { Percent }\end{array}$ & $P_{a}$ & -0.003 & 0.001 & -0.153 & -4.202 & 0.000 \\
\hline
\end{tabular}

The data reported in Table 6.4 shows the linear regression equation coefficients for the various model variables. The "B" values are the coefficients for each variable. These are the values that the variable's data should be multiplied by in the final linear equation for predicting the HERPI. The "Constant" is the intercept equivalent in the equation. The Significance (Sig.) should be 0.05 or less to be significant at 95 percent. As shown in Table 6.4, there are three models, the "best" one -variable, two-variable and three-variable model. The adjusted $\mathrm{R}^{2}$ value of $0.578,0.859$ and 0.880 gives the most useful measure of the success of the model, respectively.

The capability of each model in predicting the HERPI is validated by comparing the HERPI predicted with the known key variable with that calculated for each cycle. The regression function is developed using data of 43 cycles noted as model training cycles. The 11 cycles left was used to evaluation cycles. As shown in Figure 6.3, the one variable $\left(\bar{\alpha}_{+}\right)$model is not able to accurately predict the HERPI of these test cycles as indicated by low $R^{2}$ of the training and evaluation cycles. The regression model with two key variables $\left(\bar{\alpha}_{+}\right.$and $\left.\bar{v}\right)$ is able to successfully predict the HERPI of the 54 cycles. The $R^{2}$ of the linear fit from training cycles and evaluation cycles were 0.86 , and 0.84 , respectively. The regression model with three key variables $\left(\bar{\alpha}_{+}, \bar{v}\right.$, and $\left.P_{a}\right)$ will do slightly better than that with two key variables $\left(\bar{\alpha}_{+}\right.$and $\left.\bar{v}\right)$. In this research, the regression model with two key 
variables $\left(\bar{\alpha}_{+}\right.$and $\left.\bar{v}\right)$ is used to evaluate the HERPI of the actual cycles. The HERPI can be calculated by the linear regression equation HERPI $=0.302+1.792 \times \bar{\alpha}_{+}-0.016 \times$ $\bar{v}$. Figure 6.4 shows the three-dimensional contour plot for HERPI as a function of $\bar{\alpha}_{+}$and $\bar{v}$. The two key driving pattern features $\left(\bar{\alpha}_{+}, \bar{v}\right)$ will be utilized as the input parameters of the driving pattern prediction model.

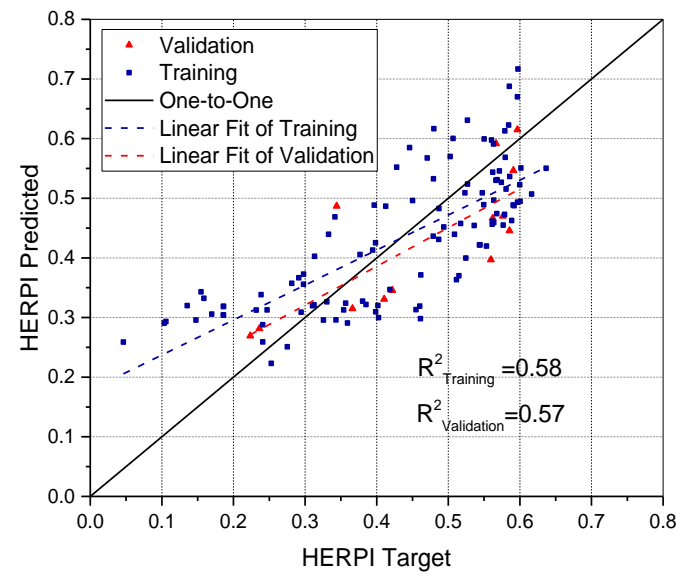

a. HERPI predicted vs. target under the variable $\bar{\alpha}_{+}$

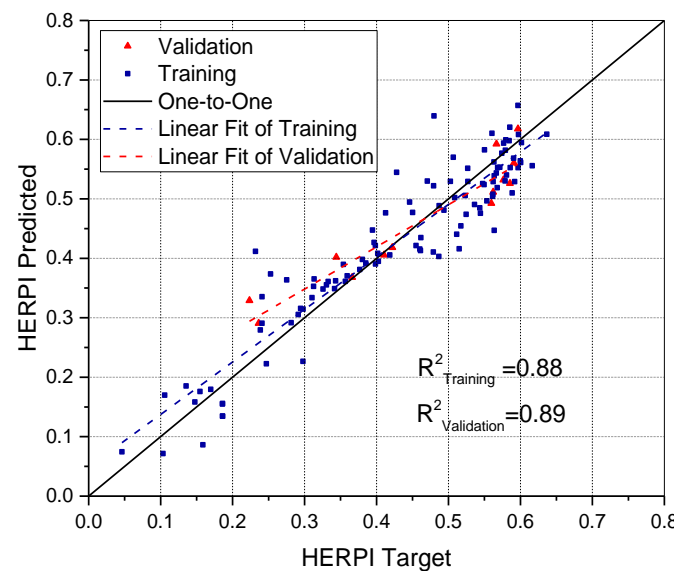

c. HERPI predicted vs. target under the variables $\bar{\alpha}_{+}, \bar{v}$, and $P_{a}$

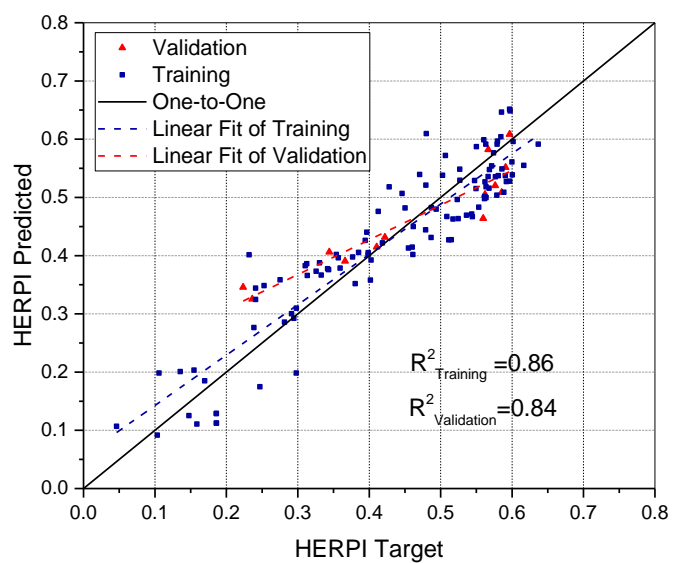

b. HERPI predicted vs. target under the variables $\bar{\alpha}_{+}$and $\bar{v}$

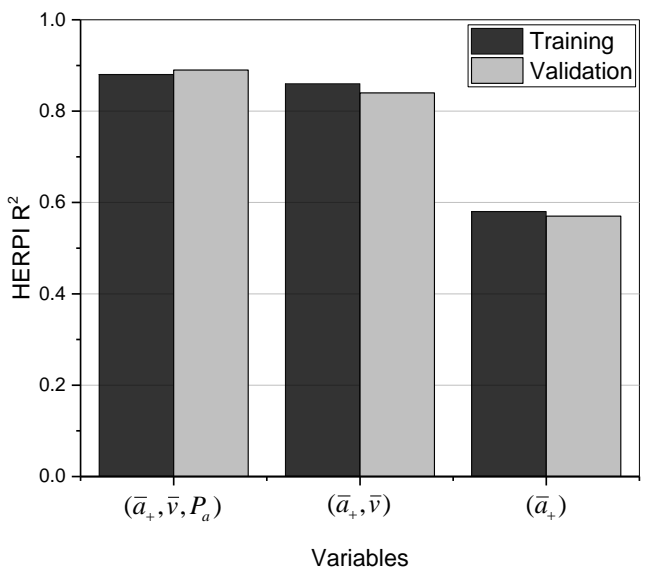

d. Effect of variables on $\mathrm{R}^{2}$ of HERPI

\section{Figure 6.3 Validation of the models under different variables}




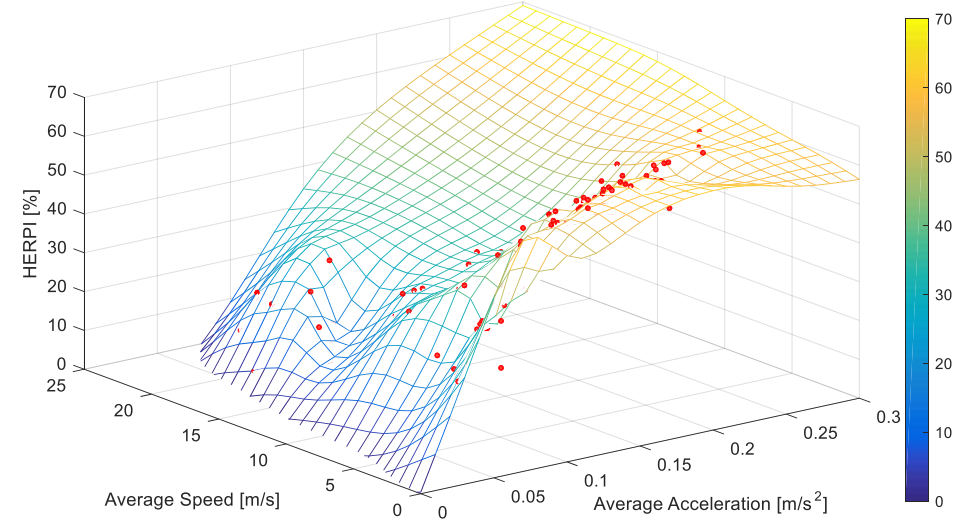

Figure 6.4 Three-dimensional contour plot of the HERPI with variables average acceleration and speed

\subsection{Representative Driving Pattern Development}

The representative driving patterns (RDP) are developed using the K-means cluster analysis, a data classification method carried out by separating the data into groups with the number of clusters pre-defined by the user (Vojnikova 2009).

\subsubsection{The Development of RDP from the 54 Heavy-duty Driving Cycles}

The 54 heavy-duty driving cycles can be classified into numerous clusters (driving patterns) using the K-means cluster model based on the Euclidean distance. In this research, the driving cycle is represented by the two key driving pattern features $\left(\bar{\alpha}_{+}, \bar{v}\right)$, which are the input variables of the K-means model. Table 6.5 shows the $\left(\bar{\alpha}_{+}, \bar{v}\right)$ and distance of the 6 representative driving cycles K-means cluster identified from the 54 heavy-duty cycles. These 6 cycles are recognized as the RDPs developed and shown in Figure 6.5. The $\left(\bar{\alpha}_{+}, \bar{v}\right)$ of these representative driving patterns will be used as the key driving features to identify the future driving patterns as shown in Table 6.6. 
Table 6.5 Cluster membership and the distance

\begin{tabular}{|c|c|c|c|c|c|}
\hline Cycle & Cluster & Distance & Cycle & Cluster & Distance \\
\hline HHDDT_Creep & 1 & 4.79 & BAC & 5 & 3.98 \\
\hline Yard & 1 & 7.79 & CSHVC & 5 & 3.99 \\
\hline NYC BUS & 2 & 0.00 & CSHVR & 5 & 3.99 \\
\hline NY_Comp & 3 & 3.46 & ECE_15 & 5 & 5.02 \\
\hline WMATA & 3 & 3.66 & ETC_Urban & 5 & 5.73 \\
\hline Liberty & 3 & 4.09 & JE05 & 5 & 6.01 \\
\hline Nuremberg_R36 & 3 & 4.15 & SORT_3_Estimated & 5 & 6.39 \\
\hline OCTA & 3 & 4.96 & Houston Bus Cycle(333) & 5 & 7.28 \\
\hline KCM4 & 3 & 5.15 & WVU_City & 5 & 7.53 \\
\hline Braunschweig & 3 & 5.51 & UDDS & 5 & 7.83 \\
\hline BEELINE & 3 & 6.70 & CBD & 5 & 8.81 \\
\hline КСM3 & 3 & 6.73 & SORT_2_Estimated & 5 & 8.87 \\
\hline MCS & 3 & 6.76 & CBD_Truck & 5 & 9.33 \\
\hline SORT_1_Estimated & 3 & 7.20 & KCM & 5 & 10.04 \\
\hline VC_28 & 3 & 7.89 & VC_274 & 5 & 10.22 \\
\hline Paris & 3 & 9.47 & CILCC & 5 & 10.98 \\
\hline KCM2 & 3 & 9.71 & Int_Local & 5 & 11.85 \\
\hline Manhattan & 3 & 10.08 & Arterial & 5 & 11.96 \\
\hline Commuter_No_Init_Idle & 4 & 1.92 & WVU_5_Peak & 5 & 16.41 \\
\hline Commuter-DieselNet & 4 & 1.93 & Freeway & 6 & 1.34 \\
\hline KCM1 & 4 & 3.74 & ETC_FIGE & 6 & 3.75 \\
\hline ETC_Rur_Mot & 4 & 4.53 & EUDC_Low & 6 & 4.92 \\
\hline Commuter & 4 & 4.86 & EUDC & 6 & 5.28 \\
\hline Commuter310 & 4 & 4.86 & HHDDT_All & 6 & 5.57 \\
\hline HWFET & 4 & 7.83 & WMATA_COMMUTER_2 & 6 & 6.96 \\
\hline HHDDT_Trans & 5 & 1.51 & HHDDT_Cruise & 6 & 7.33 \\
\hline ROUTE77 & 5 & 2.31 & VC_637 & 6 & 10.47 \\
\hline
\end{tabular}

\section{Table 6.6 Identification features of six RDPs}

\begin{tabular}{|c|c|c|c|c|c|c|}
\hline \multirow{2}{*}{$\begin{array}{c}\text { Key driving pattern } \\
\text { parameters }\end{array}$} & \multicolumn{7}{|c|}{ Cluster Center } \\
\cline { 2 - 8 } & $\mathbf{1}$ & $\mathbf{2}$ & $\mathbf{3}$ & $\mathbf{4}$ & $\mathbf{5}$ & $\mathbf{6}$ \\
\hline Representative Driving Cycle & $\begin{array}{c}\text { HHDDT } \\
\text { Creep }\end{array}$ & NYC BUS & NY Comp & $\begin{array}{c}\text { Commuter } \\
\text { No Init Idle }\end{array}$ & $\begin{array}{c}\text { HHDDT } \\
\text { Trans }\end{array}$ & Freeway \\
\hline Average speed [mph] & 2.55 & 3.68 & 11.26 & 44.71 & 15.32 & 34.93 \\
\hline Average acceleration [g] & 0.005 & 0.015 & 0.02 & 0.008 & 0.015 & 0.009 \\
\hline
\end{tabular}



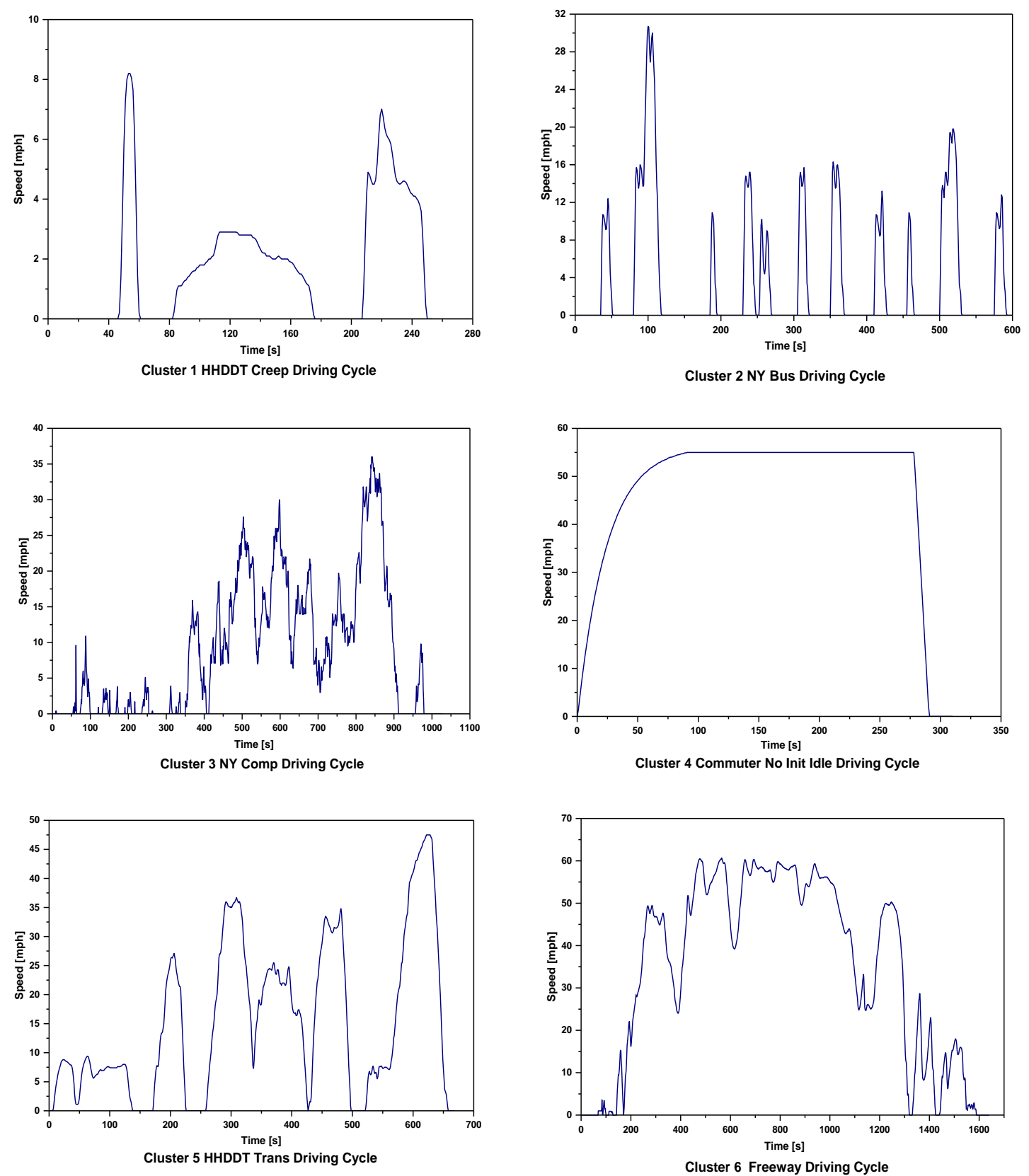

Figure 6.5 Six representative standard driving cycles

\subsection{The Short Term Prediction for Driving Pattern Features}

Short term driving pattern prediction is defined as the process of estimating the anticipated driving patterns in the short term future with the known historical and current traffic information (Vlahogianni, Golias and Karlaftis 2004). The short term (also called segment) traffic information can be categorized into a time based segment and a distance based segment. 


\subsubsection{The Short Term Prediction Based on Distance Segment}

Numerous sources in the literatures (Van Lint and Hinsbergen 2012, Murphey, et al. 2008, Xu, et al. 2009) have reported the prediction of the vehicle operation features using the time based segment and the prediction was conducted using the standard emissions test cycles. Although operated on a fixed route, the operation characteristics of the transit bus over each segment at different times are not unique but plural.

In this research, the transit route is divided into distance-based segments. Figure 6.6 shows the average velocity of transit bus operation over each distance-based segment. The horizontal axis represents the distance from $675 \mathrm{~m}$ to $1125 \mathrm{~m}$. This piece of the route is divided into ten even segments with $45 \mathrm{~m}$ in each segment. The average velocity of each segment can be calculated. In this research, the RDP of the distance based segment are the inputs of the prediction model, which provide better results, and more useful information than the time based segment.

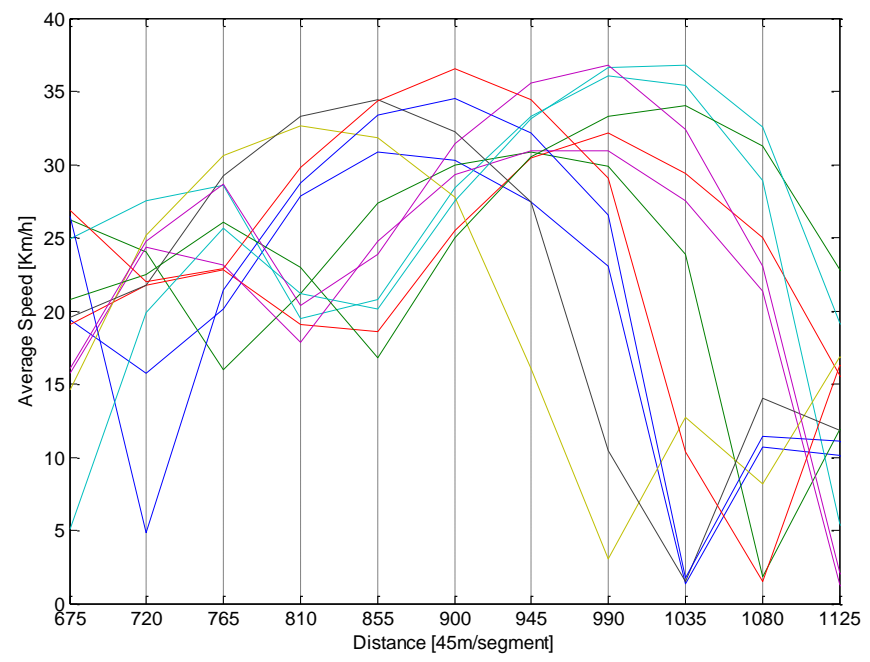

Figure 6.6 The distance based segment

The mathematical statement of the short-term driving pattern predicted at position $d$ can be expressed as follows:

$$
\hat{x}(d+D)=f\{x(d), x(d-D), x(d-2 D)\}
$$

Equation 6.5 
Where, $D$ is the distance segment of the prediction, $x(d), x(d-D)$ and $x(d-2 D)$ are the measured driving pattern features of current and past distance based segments, and $\hat{x}(d+D)$ is the predicted features at the $d+D$ horizon.

Figure 6.7 illustrates the prediction of the travel distance by means of a distance-space diagram. From the start of the look ahead window, the past three driving pattern features at window in $1\{x(d), x(d-D), x(d-2 D)\}$ as the inputs of prediction model, the predicted features at window out $1\{\hat{x}(d+D)\}$. Due to disturbances and model-plant mismatch, the actual driving features are different from the predicted ones. So the next input window will be implemented only until the next measurement becomes available from the vehicle ECU or GPS. The prediction will continue to next window out $2\{\hat{x}(d+$ $2 D)\}$ with the input window in $2\{x(d-D), x(d-2 D), x(d-3 D)\}$. The entire route can be predicted.

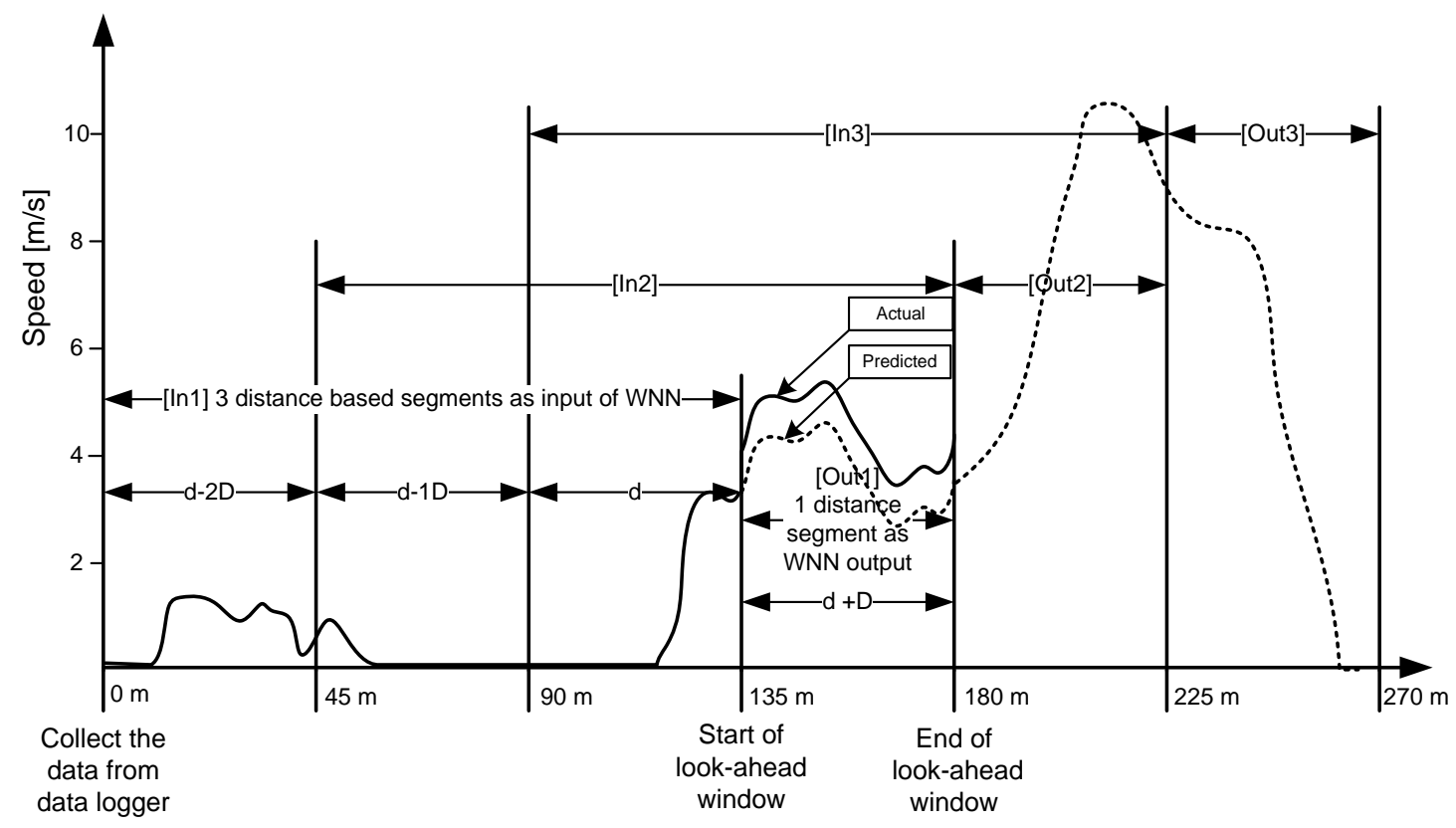

Figure 6.7 Illustration of the distance based segment prediction diagram 


\subsubsection{The Wavelet Neural Network}

Wavelet neural networks (WNN) combines the theory of wavelets and neural networks into one. A WNN model generally consists of a feed-forward neural network and one hidden layer with the activation functions drawn from an orthonormal wavelet (Chan, Dillon and Singh, et al. 2012).

A WNN is more efficient than the conventional neural networks in approximating nonlinearities appearing in servo systems (Falamarzi, et al. 2014). Benefiting from the wavelet transform localization properties and self-studying of artificial neural networks, a WNN possesses improved capacity of approximation and the training times are significantly reduced.

Figure 6.8 shows the architecture of the WNN model. The model is a three-layer structure, consisting of an input neuron layer $\mathrm{x}(\mathrm{i})$, a hidden neuron layer, and an output neuron layer $\mathrm{y}(\mathrm{k})$. The connections between hidden neurons and output neurons are named weights $\omega_{\mathrm{i} \rightarrow \mathrm{h}}$, and $\omega_{\mathrm{h}-\mathrm{k}}$, respectively. The input data is directly transmitted into the wavelet nodes in the hidden neuron layer. The discrete wavelet transform is described as follow:

$$
\Psi_{a, b}(x)=|a|^{\frac{1}{2}} \Psi\left(\frac{x-b}{a}\right)
$$

Equation 6.6

Where $\mathrm{a}$ is the dilation parameter and $\mathrm{b}$ is the translated parameter.

The parameter $\mathrm{n}$ is the number of input-dimensions and $\mathrm{m}$ is the number of the wavelets. The output function is described by the following formula:

$$
y(k)=\sum_{j}^{m} \omega_{h-k} \Psi_{j}(x)
$$


Where $\Psi_{j,} j=1, \ldots m$ is used as a nonlinear transformation function of hidden nodes, and $\omega_{\mathrm{h}-\mathrm{k}}, \mathrm{j}=1, \ldots \mathrm{m}$ is used as the adjustable weighting parameters to provide the function approximation.

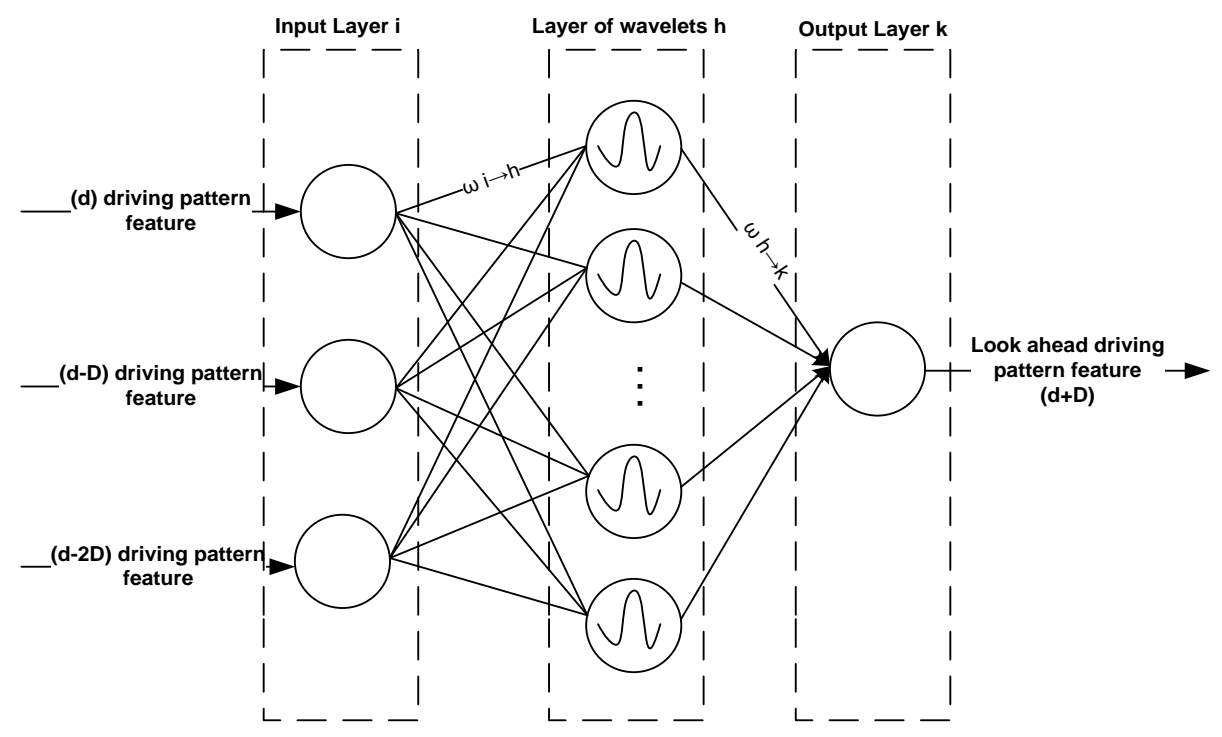

Figure 6.8 The architecture of the WNN model

In this WNN model, the neural network simulator is described as follows:

(1) To initialize the parameters

The dilation parameter $a$, translation parameter $b$ and the neuron connection weights $\omega_{\mathrm{i} \rightarrow \mathrm{h}}$, $\omega_{\mathrm{h}-\mathrm{k}}$ are initialized to random values $[0,1]$;

(2) To normalize the input and target output

The inputting vector data $x(i)$ is normalized and the corresponding output vector values $y(k)$ fall in the range $[-1,1]$

(3) To reduce the error between the desired and actual output

$$
e=\sum_{k=1}^{m} \dot{y}(k)-y(k)
$$

Equation 6.8

Where $y(k)$ is the desired output and $y(k)$ is the WNN actual output. 
The error can be reduced by adjusting $\omega_{\mathrm{i} \rightarrow \mathrm{h}}, \omega_{\mathrm{h}-\mathrm{k}}$, a, and $\mathrm{b}$.

(4) Error criteria $\delta$

Step (3) is repeated until $e$ satisfies the given error criteria $\delta$, and the training process of the WNN is completed.

\subsubsection{Case Study of the Future Driving Feature Prediction}

The model was applied to predict the operation velocity of one session of Route 6 in Southeast Area Transit (SEAT) district of Preston, CT. The average speed is one of the key driving pattern features. The average speed and average acceleration predicted using the WNN with the selected distance segment can be used as the input of the driving pattern identification model. Figure 6.9 shows the average speed predicted using four segment

distances. The distance of the segment selected was found to significantly affect the acuracy of the predicted average velocity. The shorter the distance of the reference segment selected, the more accurate the predicted average speed was. 


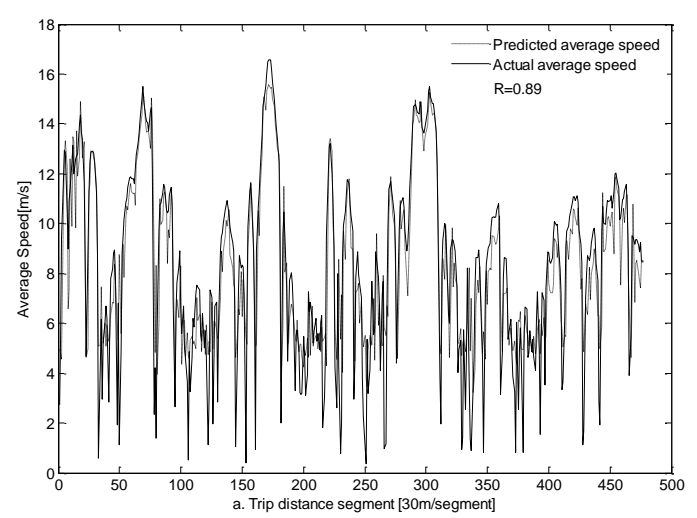

(a) predicted with distance segment of $30 \mathrm{~m}$

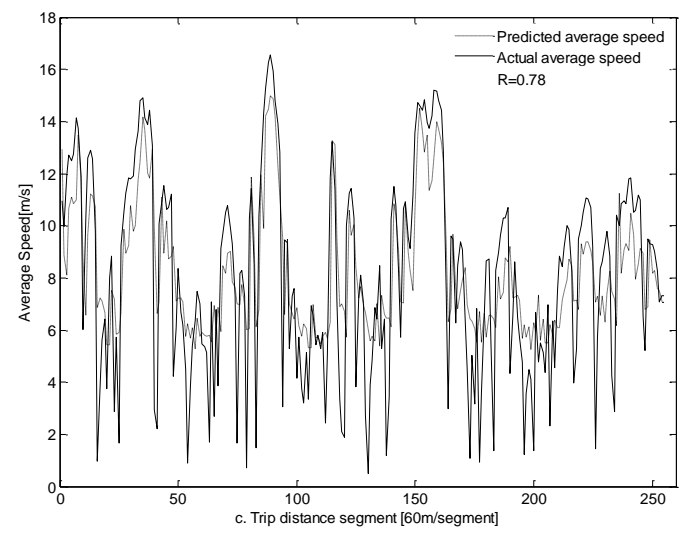

(c) predicted with distance segment of $60 \mathrm{~m}$

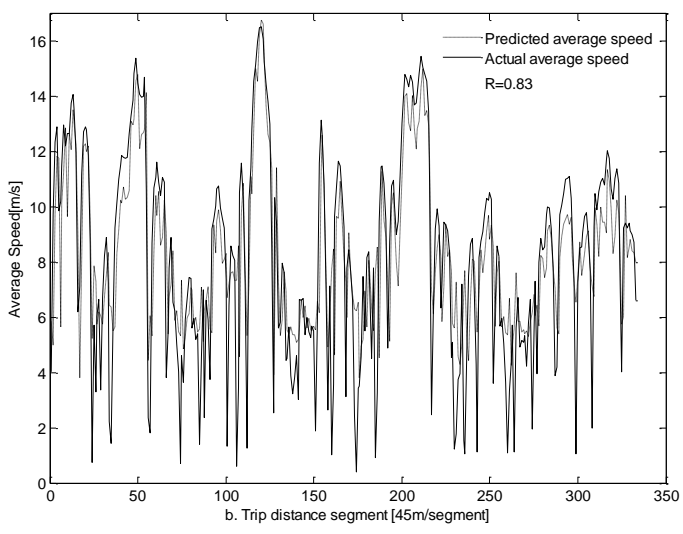

(b) predicted with distance segment of $45 \mathrm{~m}$

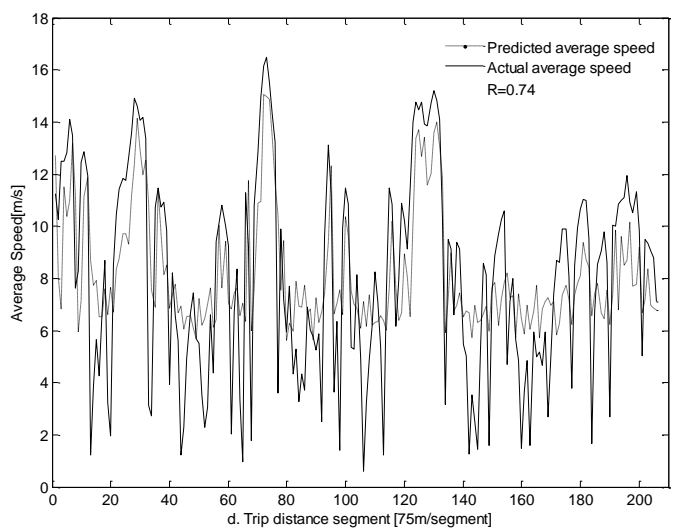

(d) predicted with distance segment of $75 \mathrm{~m}$

Figure 6.9 The average speed predicted using different distance segment

\subsubsection{Driving Pattern Identification Model}

The predicted key driving pattern features are of different orders of magnitude. In this research, the predicted driving pattern features are normalized. The normalized features will serve as the inputs of the driving pattern identification model. The six representative driving patterns will be used as initial candidate patterns. The corresponding identification features are extracted using the k-means cluster center and stored in the identification model. Figure 6.10 shows the architecture of the driving pattern identification model. The model predicts the driving features of the coming distance segment with the known features of the prior two segments. 


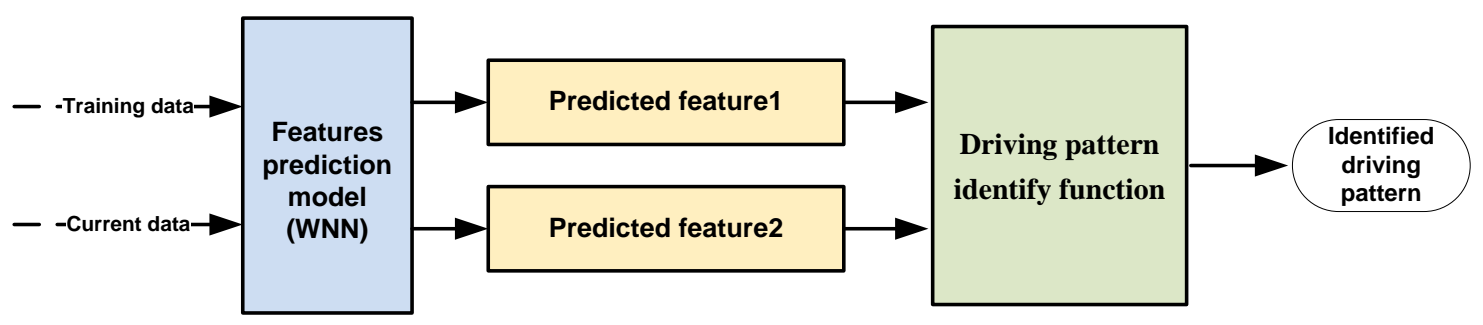

Figure 6.10 The architecture of the driving pattern identification model

The driving pattern identification model identifies the driving patterns by comparing the predicted driving features with that of the representative driving patterns using the following equation.

$$
\begin{gathered}
C_{k}=\min \left\{\left|\bar{v}_{p}-\bar{v}_{k}\right|+\left|\bar{\alpha}_{+, p}-\bar{\alpha}_{+, k}\right|\right\} \\
k=1,2, \ldots 6
\end{gathered}
$$

Where ${ }_{p}$ is the normalized predicted future driving features, ${ }_{k}$ is the normalized identification features from the 6 candidates driving patterns, and $C_{k}$ is the predicted driving pattern.

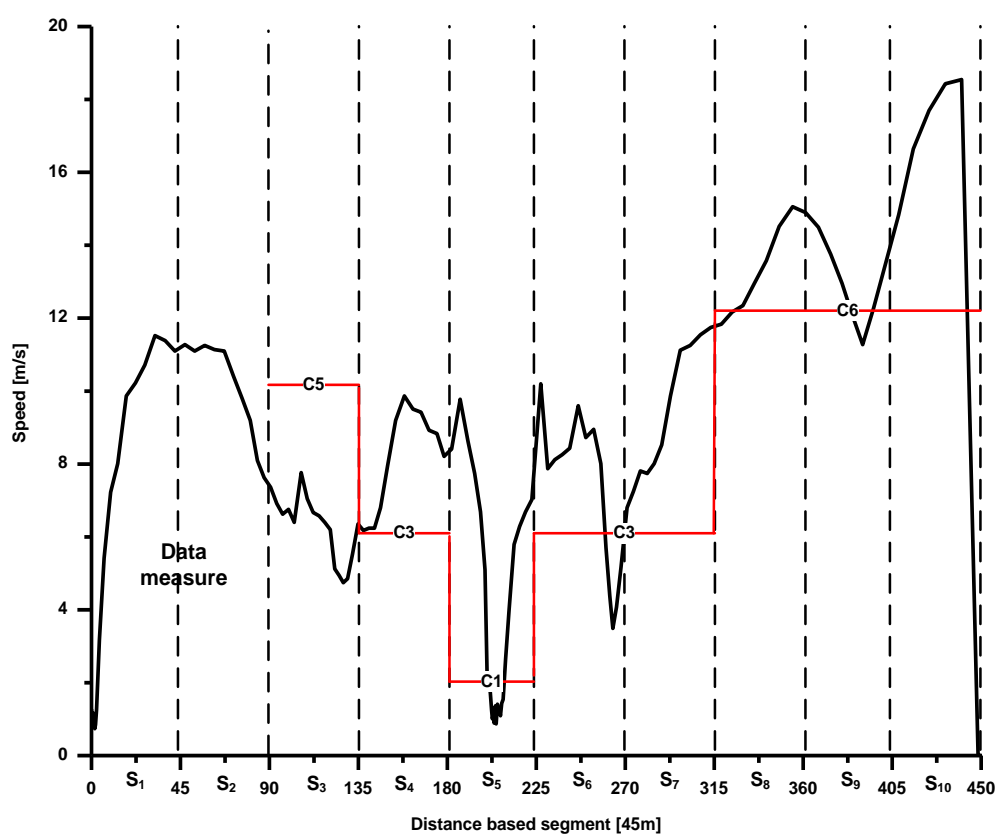

Figure 6.11 The identified driving patterns of one session from Rt6 
The driving pattern identification model will classify the current driving pattern into one of the six representative standard driving cycles (driving patterns), then the appropriate average $\lambda$ or $\lambda$ maps will be utilized accordingly. Figure 6.11 shows identified driving patterns of one session of Route 6 in SEAT district of Preston, CT.

\subsection{Summary}

This chapter presents the development of the driving pattern prediction model capable of identifying the upcoming driving patterns based on the driving features predicted. The key driving parameters were derived using principal component analysis and stepwise regression. The latter examined the features dominating the proposed HERPI, an index proposed to evaluate the potential benefit of a typical cycle or operation over a route achievable by recovering the brake energy. The average acceleration and speed were selected as the two key driving pattern features and utilized as the input parameters of driving pattern prediction model. The RDPs were developed using the K-means cluster model. The examination of the current 54 heavy-duty driving cycles leads to the development of 6 RDPs including the NYC Bus, Freeway, NY Comp, Commuter No Init Idle, HHDDT Creep, and HHDDT Trans.

A Wavelet neural network (WNN) was used in the short term driving features prediction model for estimating the driving pattern features with the known historical and current traffic information. The shorter the distance of the reference segment selected, the more accurate the predicted average speed was. The predicted driving pattern features will serve as the inputs of the driving pattern identification model, which compares the predicted future driving pattern features with the identification features from the 6 candidate driving patterns and derives the driving pattern of the upcoming distance segment in the transit route. 


\section{Adaptive ECMS Based on ECMSwDP with Driving Pattern Identification Model}

As reported in the previous chapters, the pattern of the driving features was shown to significantly affects the optimal $\lambda$ derived using the ECMSwDP approach. However, the optimal solution of an ECMSwDP can be developed only when the driving cycle is

available. Such a limitation can be solved by the proposed adaptive ECMS (A-ECMS) with $\lambda$ derived using the driving pattern identified, which provides the suboptimal control strategies of a hybrid bus. In this research, the success of the A-ECMS in developing the suboptimal control strategies is examined by observing the extent of approximation of the FC achieved with A-ECMS to the optimal one observed with the ECMSwDP. The simulation results will be analyzed and discussed.

\subsection{Structure of the Proposed Controller}

Figure 7.1 depicts the structure of the suboptimal controller developed using the A-ECMS with the $\lambda$ derived using the predicted driving pattern. The controller can be divided into two parts: (1) the off-line calculation which is able to generate the optimal $\lambda$ maps or the average $\lambda$ value based on the representative driving patterns; (2) the real-time controller using A-ECMS is going to utilize the $\lambda$ identified by the the off-line calculation and the future driving patterns based on the predicted driving features. 


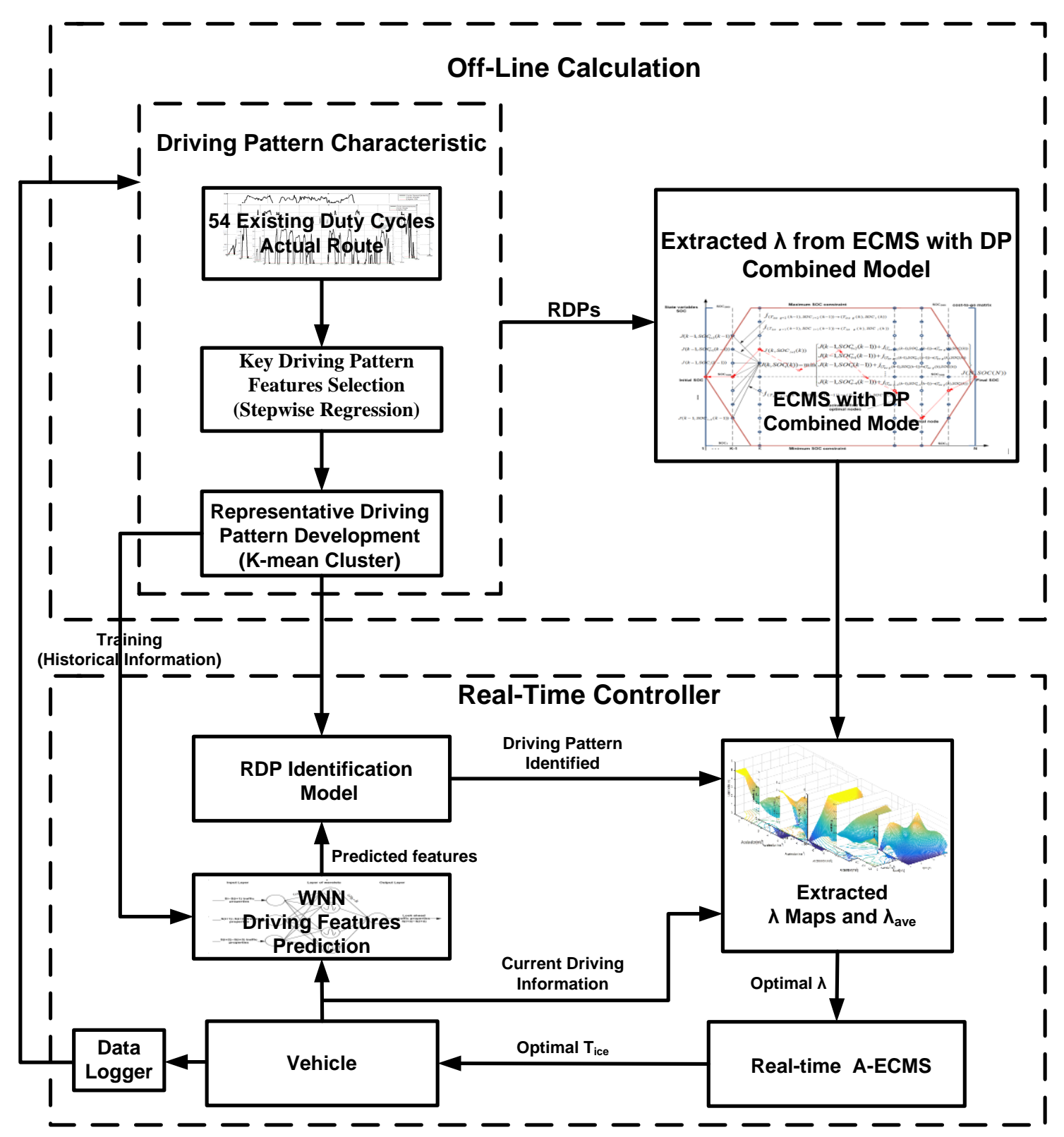

Figure 7.1 Flow chart of real-time optimal control strategy of hybrid transit bus based on predicted driving patterns

\subsection{Adaptive ECMS}

As reported in the past chapters, the $\lambda$ plays a crucial role for the battery to keep the sustainable charging requirement, and achieve the optimal fuel economy. The solution is extremely sensitive to the value of $\lambda(t)$, which can be tuned appropriately with the known knowledge of the routes and vehicle operating features. To achieve optimal results, one 
algorithm is suggested to adjust $\lambda$ based on feedback from the SOC and past or future driving conditions while maintaining the battery charge sustainability of the entire driving cycle. The proposed A-ECMS is an algorithm with $\lambda$ refined according to the feedback of the known or predicted vehicle operating conditions (Musardo, et al. 2005, Onori, Serrao and Rizzoni 2010, Onori and Serrao 2011).

Figure 7.2 shows the schematically SOC trajectories derived using ECMS with constant $\lambda$ and A-ECMS with $\lambda$ varied with vehicle operating conditions, respectively. The A-ECMS decreased the $\lambda$ to be lower than the constant $\lambda$ of the ECMS and depleted the battery SOC to its lower limit at the end of the uphill operation. This made it possible for the battery to absorb more electrical energy made available during the brake process from the upcoming downhill operation.

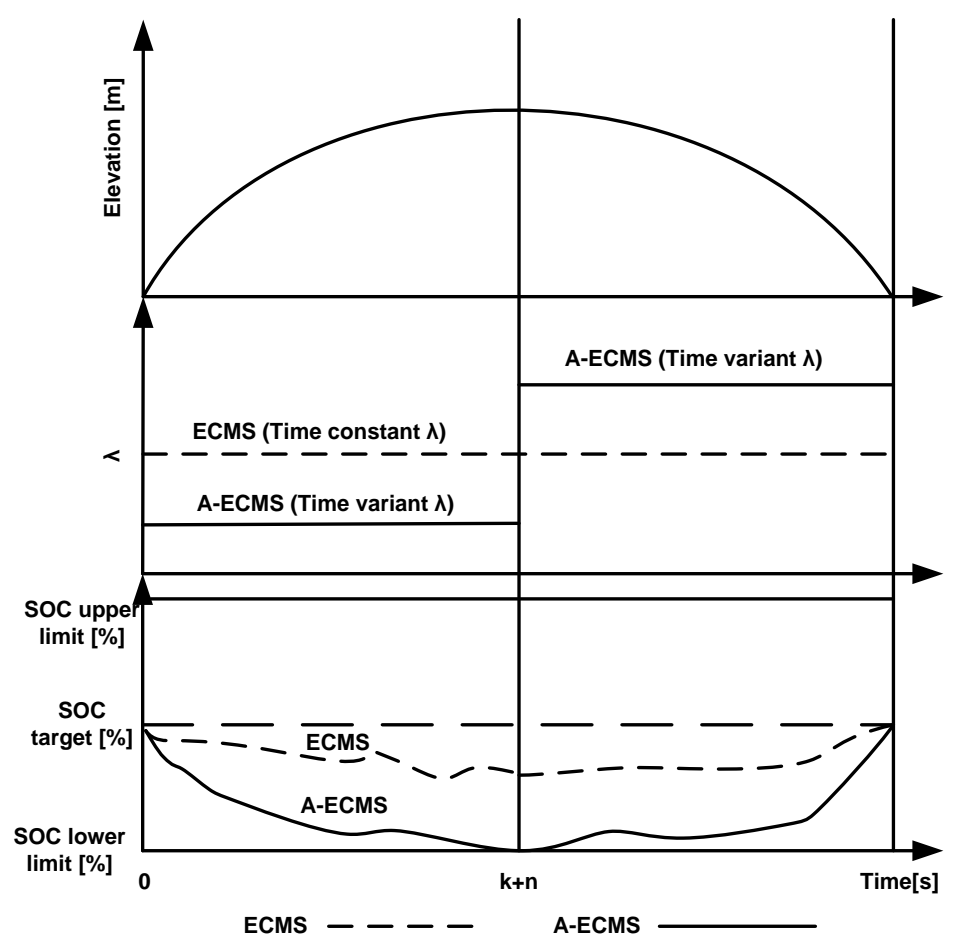

Figure 7.2 The schematic diagram of SOC trajectories derived using ECMS and A-ECMS 
There are a few adaptation methods, which include: (1) adaptation based on feedback from SOC; (2) adaptation based on $\lambda$ maps; and (3) adaptation based on driving pattern predicted.

The previously described ECMS with penalty function in chapter 4 is one method of the A-ECMS based on SOC feedback. This feedback controller dynamically modifies the $\lambda$ values to maintain the SOC variation close to the reference SOC with the penalty Equation 4.9. Figure 7.3 shows the schematic diagram of A-ECMS based SOC.

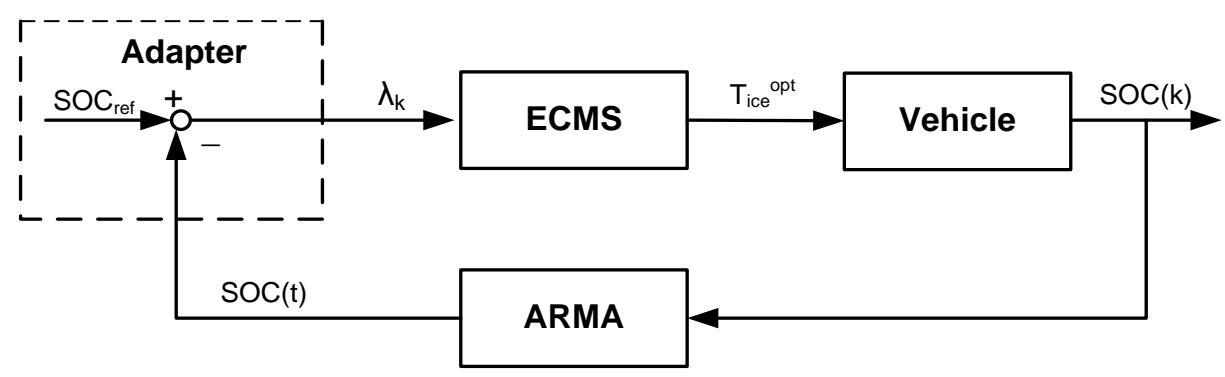

Figure 7.3 A-ECMS based on SOC feedback

\subsubsection{The A-ECMS Based on $\lambda$ Maps from the Vehicle Operation Data}

Figure 7.4 shows the A-ECMS based on $\lambda$ map. In the adapter, the $\lambda$ map is generated with the optimal $\lambda$ trajectories of a known representative driving pattern derived using the ECMSwDP.

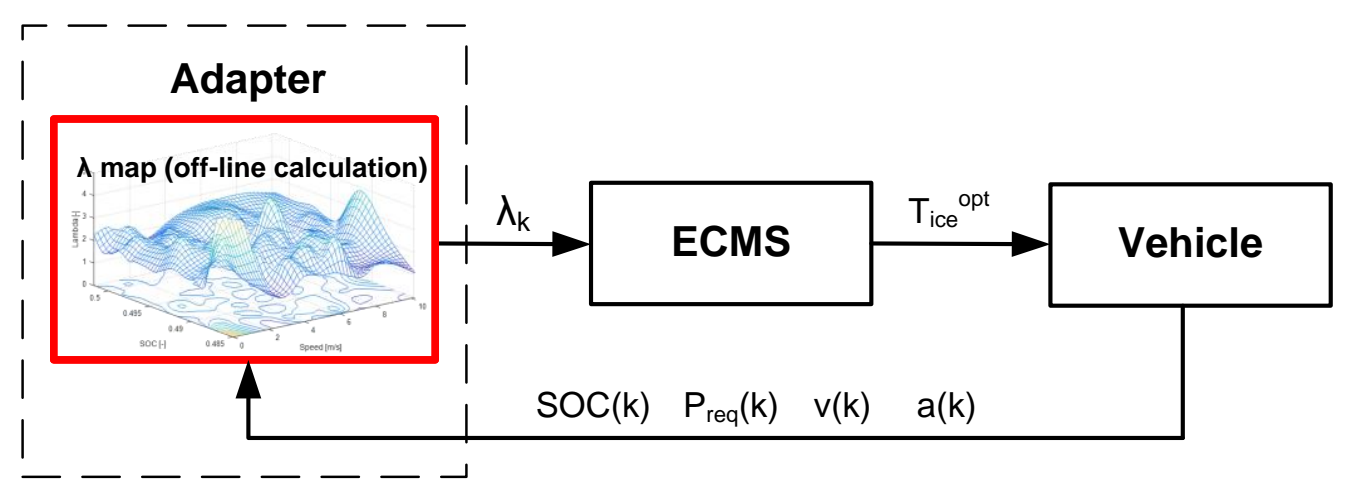

Figure 7.4 A-ECMS based on $\lambda$ map 
In the $\lambda$ map, there is a unique $\lambda$ for each given $\operatorname{SOC}(k)$, power request $P_{\text {req }}(k)$ or driving features such as vehicle speed $v(k)$ and acceleration $a(k)$ from the off-line calculation using the ECMSwDP. The consequent real-time solution is solved online since the $\lambda$ maps are derived using the global optimal method. Such a solution can be considered as a suboptimal solution comparable to the optimal one derived using DP, which provides the best solution.

The creation of the $\lambda$ map: The $\lambda$ values derived using ECMSwDP model is further processed to derive the $\lambda$ map, which is a function of SOC and given driving pattern features. In this research, the Originpro 2015 software is utilized to build the $\lambda$ maps. The gridding methods are Kriging correlation and thin plate spline for data interpolation and smoothing. Figure 7.5 shows the $\lambda$ maps and contours as a function of SOC, speed, power request and acceleration obtained by ECMSwDP over the Beijing cycle. In the maps, the Not-a-Numbers (NaNs) are replaced by the average lambda $\lambda_{\text {ave }}$ from the extracted $\lambda(t)$ of the ECMSwDP.

As shown in Figure 7.5-a, the $\lambda$ at low speed is close to zero. It is evident that the ECMSwDP model aggressively discharges the battery to propel the vehicle using the electric motor in the Beijing cycle simulated. As shown in Figure 7.5-b, $d$ and $f$, the $\lambda$ observed within the negative power request zone (representing the braking process), is very high, indicating the preference for charging battery. This is consistent with the high $\lambda$ value observed when the acceleration rate is negative as shown in Figure 7.5-c and e. 


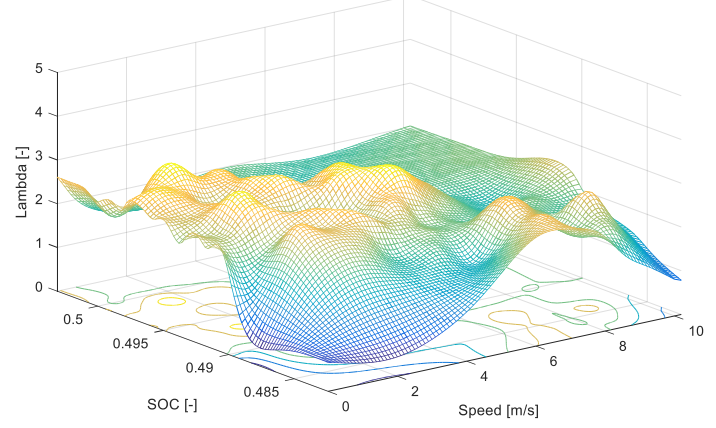

a. $\lambda(\boldsymbol{S O C}, \boldsymbol{v})$

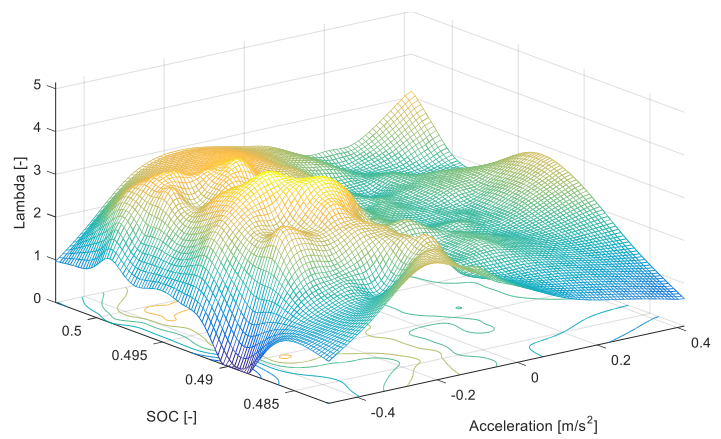

c. $\lambda(S O C, a)$

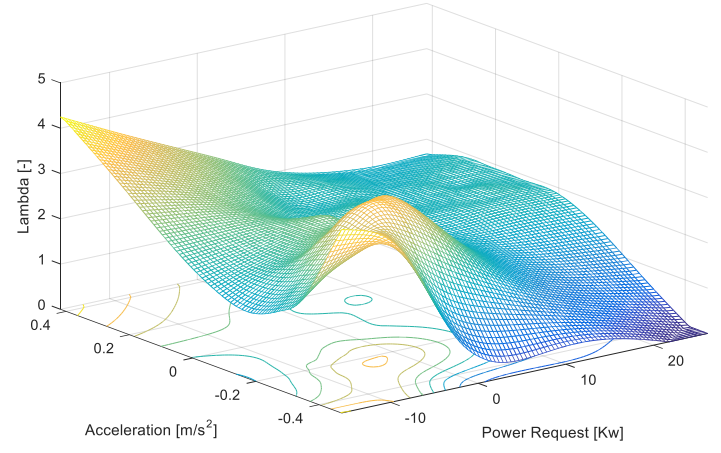

e. $\lambda(\boldsymbol{a}, \boldsymbol{v})$

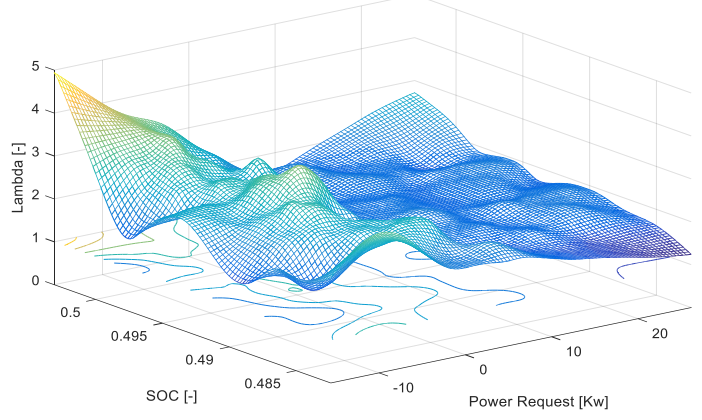

b. $\lambda\left(S O C, P_{\text {req }}\right)$

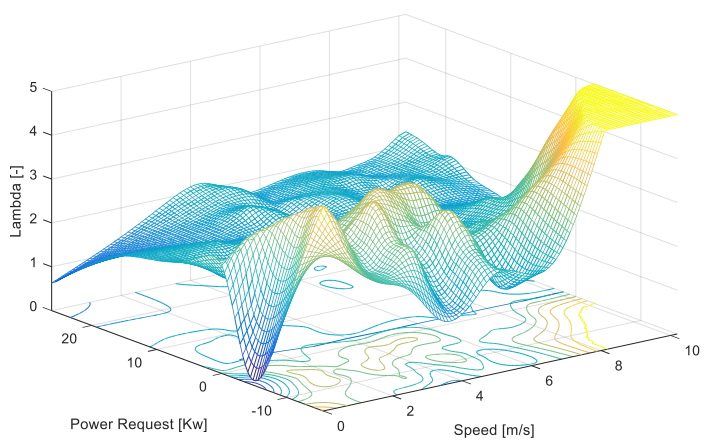

d. $\lambda\left(P_{r e q}, v\right)$

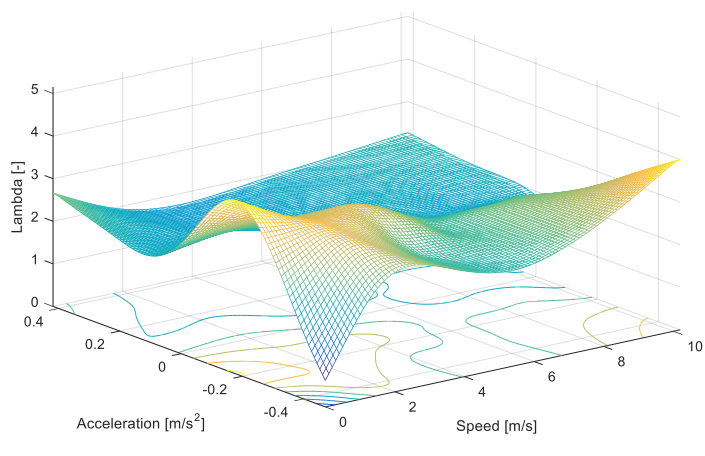

f. $\lambda\left(a, P_{r e q}\right)$

Figure 7.5 The $\lambda$ maps as a function of $S O C, v, P_{r e q}$ and $a$ derived using ECMSwDP over the Beijing cycle

\subsubsection{The Study of A-ECMS Based on Different $\lambda$ Map Functions}

The A-ECMS extracts the $\lambda$ value from the above six $\lambda$ maps created using ECMSwDP with two of the four known parameters including the current vehicle speed, power request, acceleration rate, and the SOC of the battery over Beijing cycle. Figure 7.6 and Figure 7.7 compare the simulated variation of the SOC and $\lambda$ of the hybrid vehicle with the A-ECMS 
based variations on $\lambda$ maps as a function of $S O C, v, P_{r e q}$ and $a$ obtained by ECMSwDP and DP over the Beijing cycle. The SOC trajectories of A-ECMS with different $\lambda$ maps are comparable. The variation of $\lambda$ based on SOC and the power request map seems smoother than that of other cases observed.
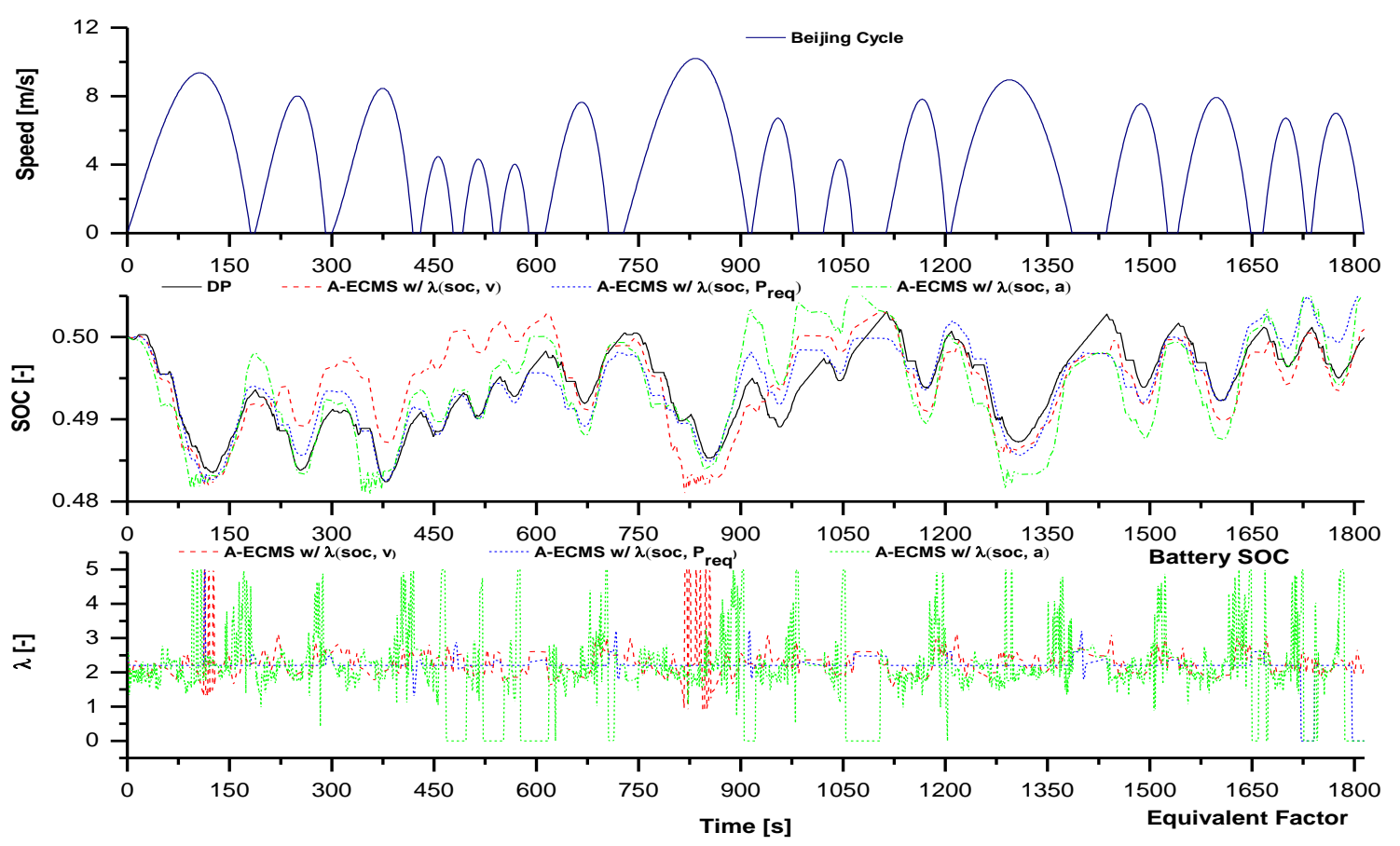

Figure 7.6 The comparison between A-ECMS based on $\lambda$ maps as a function of (SOC, $v$ ), $\left(S O C, P_{r e q}\right)$ and $(S O C, a)$ obtained and DP simulation results over the Beijing cycle 

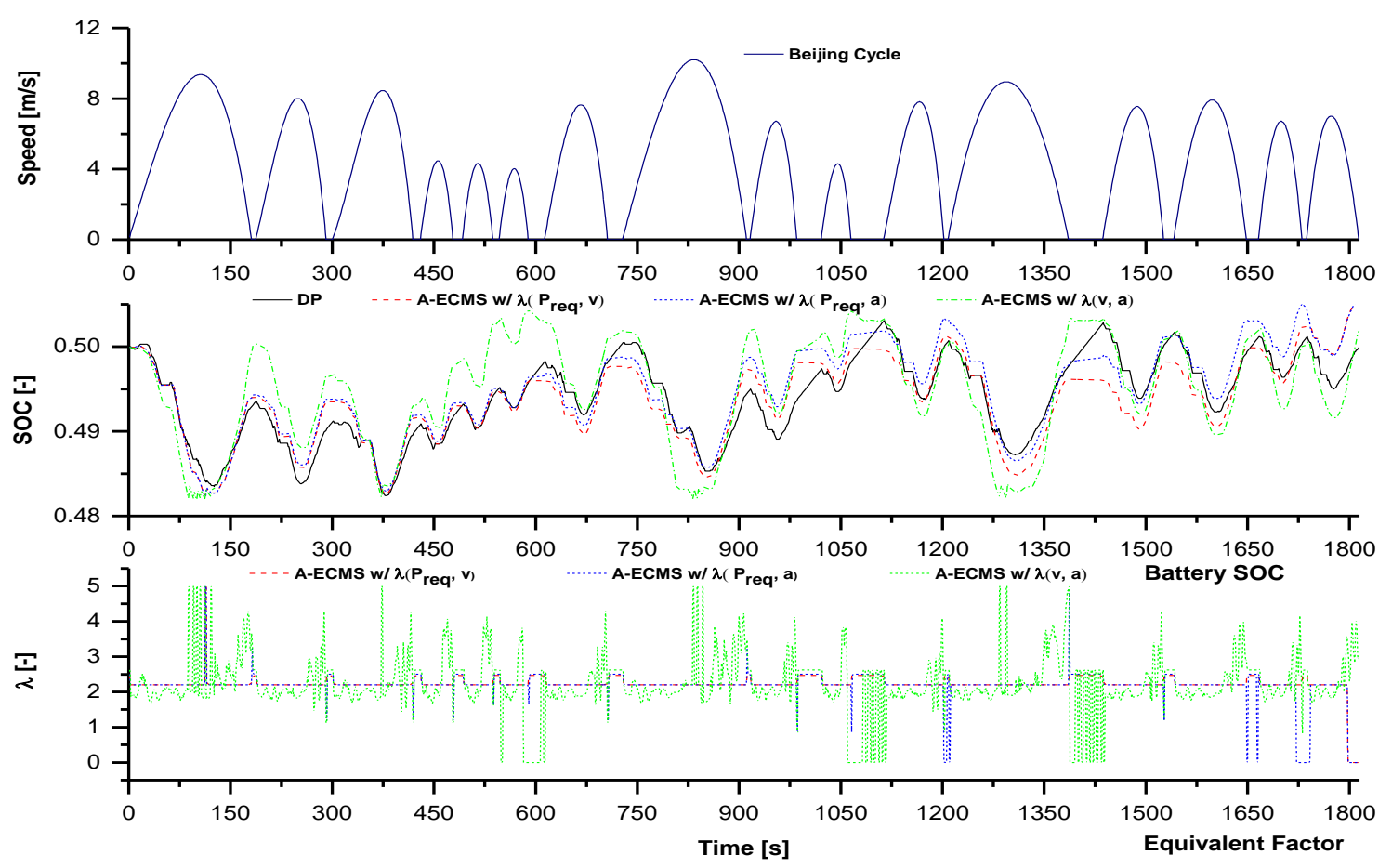

Figure 7.7 The comparison between A-ECMS based on $\lambda$ maps as a function of $\left(v, P_{\text {req }}\right)$, $\left(P_{r e q}, a\right)$ and $(v, a)$ obtained and DP simulation results over the Beijing cycle

Table 7.1 compares the fuel economy and changes in SOC of the Beijing cycle operation with A-ECMS using $\lambda$ from different $\lambda$ map functions. The battery charge is to some extent sustainable as the $\triangle \mathrm{SOC}$ is relatively small. The fuel economy observed using A-ECMS with $\lambda(v, a), \lambda\left(P_{r e q}, a\right), \lambda\left(P_{r e q}, v\right)$ are comparable and about $5 \%$ gap to that observed with DP, the best fuel economy achievable in the Beijing cycle. In comparison, the fuel consumption improvement observed with $\lambda$ involving SOC and one of the three vehicle operation parameters $\left(v, a\right.$, and,$\left.P_{r e q}\right)$ was slightly less than that based on other maps. The power request is a function of velocity and acceleration for a specific vehicle. The $\lambda$ map referring to velocity and acceleration may be considered as the suitable pair for the AECMS control strategies developed. 
Table 7.1 The fuel economy and changes in SOC observed using A-ECMS with $\lambda$ maps as a function of two of the four parameters including $S O C, v, P_{r e q}$ and $a$ over the Beijing cycle

\begin{tabular}{|c|c|c|c|c|}
\hline & FE (mpg) & $\Delta \mathrm{SOC}$ & $F E_{\text {corr }}(\mathrm{mpg})$ & Gap to DP \\
\hline A-ECMS w/ $\lambda(S O C, v)$ & 10.48 & $0.01 \%$ & 10.49 & $6.09 \%$ \\
\hline A-ECMS w/ $\lambda\left(S O C, P_{\text {req }}\right)$ & 10.32 & $0.49 \%$ & 10.41 & $6.80 \%$ \\
\hline A-ECMS w/ $\lambda(S O C, a)$ & 9.84 & $0.52 \%$ & 9.90 & $11.37 \%$ \\
\hline A-ECMS w/ $\lambda\left(P_{r e q}, v\right)$ & 10.46 & $0.57 \%$ & 10.56 & $5.46 \%$ \\
\hline A-ECMS w/ $\lambda\left(P_{\text {req }}, a\right)$ & 10.45 & $0.56 \%$ & 10.53 & $5.73 \%$ \\
\hline A-ECMS w/ $\lambda(v, a)$ & 10.57 & $0.19 \%$ & 10.61 & $5.01 \%$ \\
\hline $\mathrm{DP}(\alpha=\beta=\gamma=0)$ & 11.17 & $0.00 \%$ & 11.17 & $0.00 \%$ \\
\hline
\end{tabular}

Note: $F E_{\text {corr }}$ is the corrected fuel economy expressed in Equation 4.26 .

This is further demonstrated using the WVU-CSI cycle as an example shown in Table 7.2.

The corrected fuel economy of this vehicle operation over the WVU-CSI cycle with different $\lambda$ maps is $12.56 \%$ to $17.74 \%$ gap to that observed using DP, while the divergence of SOC derived from A-ECMS based on multiple $\lambda$ maps ranges from $-5.16 \%$ to $5.87 \%$. Table 7.2 shows the large deviation of corrected $F E_{\text {corr }}$ and the convergence of SOC. This may be due to the creation of the $\lambda$ map based on the gridding methods for data interpolation and smoothing.

As shown in Table 7.1 and Table 7.2, the best fuel economy was observed with A-ECMS using the $\lambda$ map as a function of vehicle speed $v$ and acceleration $a$ while maintanaining the sustainable SOC of the bettery. The $\lambda(v, a)$ map is utilized in the A-ECMS approach.

Table 7.2 The fuel economy and changes in SOC observed using A-ECMS with $\lambda$ maps as a function of two of the four parameters including $S O C, v, P_{r e q}$ and $a$ over the WVU-CSI cycle

\begin{tabular}{|c|c|c|c|c|}
\hline & FE(mpg) & $\Delta \mathrm{SOC}$ & $F E_{\text {corr }}(\mathrm{mpg})$ & Gap to DP \\
\hline$\overline{A-E C M S ~ w / ~} \lambda(S O C, v)$ & 9.99 & $1.86 \%$ & 10.06 & $14.60 \%$ \\
\hline$\overline{A-E C M S ~ w / ~} \lambda\left(S O C, P_{\text {req }}\right)$ & 10.02 & $-5.16 \%$ & 9.72 & $17.49 \%$ \\
\hline A-ECMS w/ $\lambda(S O C, a)$ & 10.00 & $5.87 \%$ & 10.20 & $13.41 \%$ \\
\hline A-ECMS w/ $\lambda\left(P_{r e q}, v\right)$ & 9.96 & $-4.81 \%$ & 9.69 & $17.74 \%$ \\
\hline A-ECMS w/ $\lambda\left(P_{\text {req }}, \boldsymbol{a}\right)$ & 11.01 & $-4.96 \%$ & 9.70 & $17.66 \%$ \\
\hline A-ECMS w/ $\lambda(v, a)$ & 10.22 & $2.20 \%$ & 10.30 & $12.56 \%$ \\
\hline $\mathbf{D P}(\alpha=\beta=\gamma=0)$ & 11.78 & $0.00 \%$ & 11.78 & $0.00 \%$ \\
\hline
\end{tabular}




\subsection{The A-ECMS Based on $\lambda$ Maps with Driving Pattern Identification}

This research proposes to develop A-ECMS based on $\lambda$ maps with the driving pattern identified by the driving pattern identification model as shown in Figure 7.8.

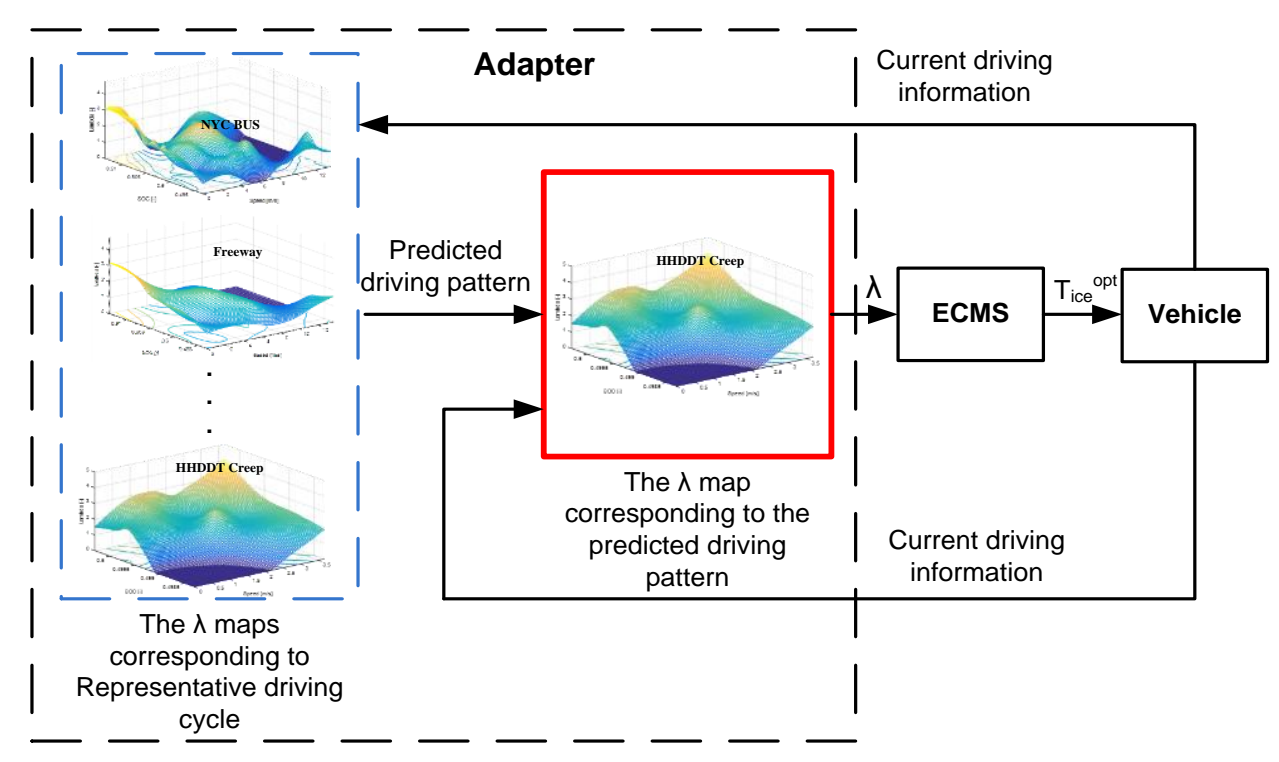

Figure 7.8 A-ECMS based on $\lambda$ maps with driving pattern identification

The key components of this approach include: (1) a driving features prediction model capable of predicting the upcoming driving features based on the past and current driving information; (2) a driving pattern identification model capable of identifying the driving pattern with the driving features predicted; (3) A-ECMS with $\lambda$ maps as a function of velocity and acceleration of the representative driving pattern. The key point of this approach is the determination of the $\lambda$ map based on the predicted driving patterns.

The past and current vehicle operation data could be made available by recording the vehicle operation data from the engine control unit (ECU) or GPS. The corresponding $\lambda$ maps can be created by the ECMSwDP and later on utilized in the real-time A-ECMS.

In the driving pattern identification model, the predicted driving features including velocity and acceleration over a short trip distance segment is compared to the created representative driving patterns. The closest driving pattern will be identified as the 
predicted representative driving pattern. The wavelet neural network will be used in the driving pattern prediction model.

\subsubsection{The $\lambda(v, a)$ Maps Corresponding to the RDPs}

Figure 7.9 shows the six maps of the $\lambda$ extracted from ECMSwDP as functions of vehicle speed $v$ and acceleration $a$ of the six representative driving cycles.

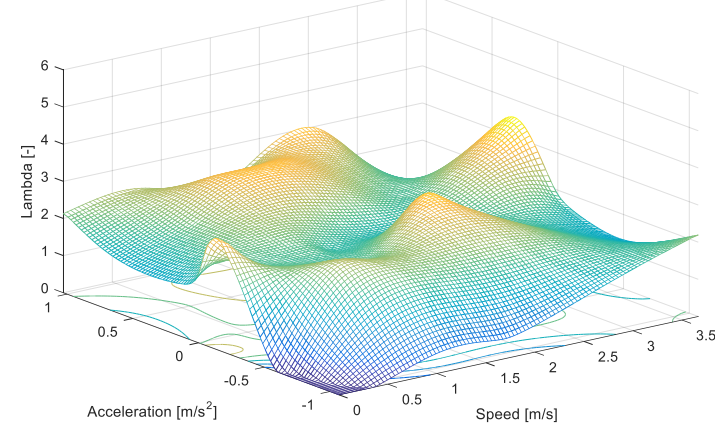

Pattern1: HHDDT Creep

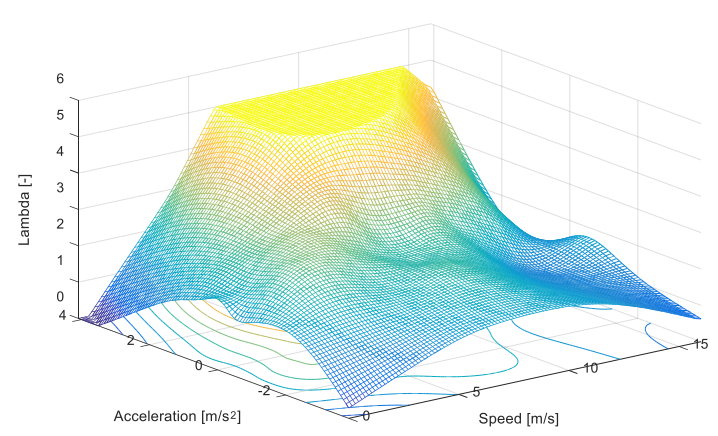

Pattern3: NY Comp

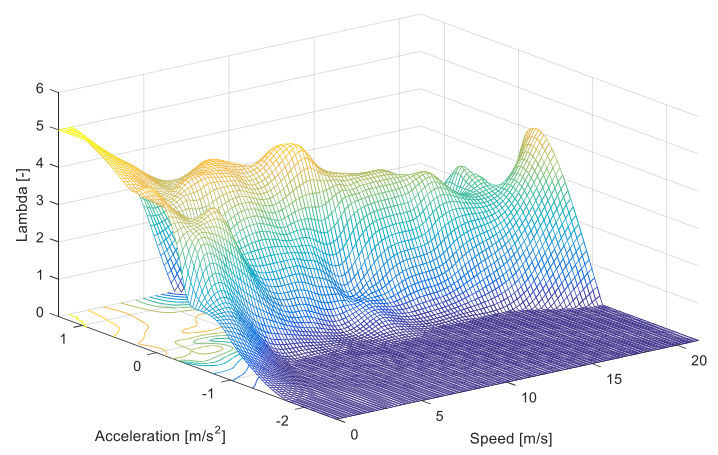

Pattern5: HHDDT Trans

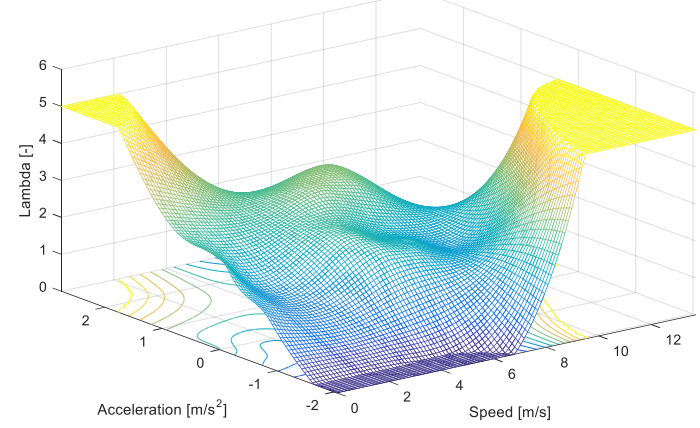

Pattern2: NYC BUS

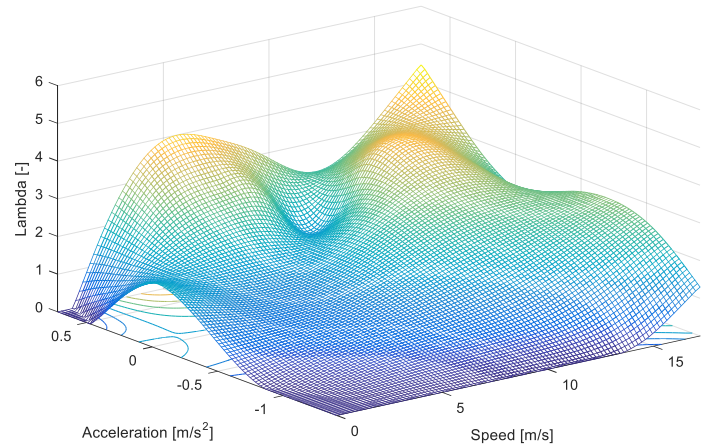

Pattern4: Commuter No Init Idle

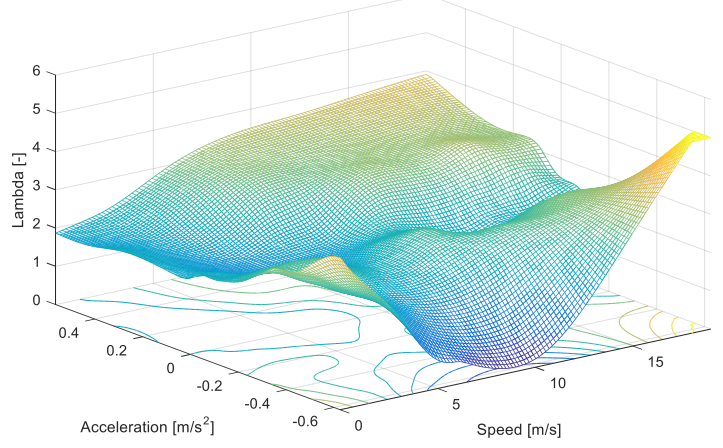

Pattern6: Freeway

Figure 7.9 $\lambda$ maps of the six RDPs 
Table 7.3 shows the fuel economy of this bus operated over the the six representative driving cycles with the A-ECMS with the $\lambda$ maps obtained by ECSMwDP. The simulated results are compared with the operation of this bus with the ECMSwDP algrithom. As shown in Table 7.3, the corrected fuel economy observed with A-ECMS with $\lambda$ maps from its own cycle is $6.28 \%$ to $14.28 \%$ gap to that observed with DP while maintianing the charge sustainability of the battery as indicated by the changes in SOC.

Table 7.3 The simulation results of A-ECMS based on its own $\lambda$ maps corresponding to the six RDPs

\begin{tabular}{|c|c|c|c|c|c|c|}
\hline \multirow{2}{*}{$\begin{array}{l}\text { Driving } \\
\text { Pattern }\end{array}$} & \multicolumn{4}{|c|}{ A-ECMS with based on its own $\lambda$ maps } & \multirow{2}{*}{$\begin{array}{c}\text { FE of DP } \\
(\mathbf{m p g})\end{array}$} & \multirow{2}{*}{ Gap to DP } \\
\hline & $\begin{array}{l}\text { Representative } \\
\text { Driving Cycle }\end{array}$ & $\begin{array}{c}\text { FE } \\
(\mathrm{mpg})\end{array}$ & $\Delta \mathrm{SOC}$ & $\begin{array}{r}F E_{\text {corr }} \\
(\mathrm{mpg})\end{array}$ & & \\
\hline Pattern1 & HHDDT Creep & 3.97 & $0.04 \%$ & 4.00 & 4.59 & $12.85 \%$ \\
\hline Pattern2 & NYC Bus & 6.65 & $0.30 \%$ & 6.83 & 7.33 & $6.82 \%$ \\
\hline Pattern3 & NY Comp & 7.85 & $-0.21 \%$ & 7.79 & 8.93 & $12.77 \%$ \\
\hline Pattern4 & Commuter No Init Idle & 9.59 & $-0.26 \%$ & 9.47 & 10.30 & $8.06 \%$ \\
\hline Pattern5 & HHDDT Trans & 8.08 & $-1.30 \%$ & 7.76 & 8.94 & $13.20 \%$ \\
\hline Pattern6 & Freeway & 13.62 & $-0.32 \%$ & 13.51 & 15.76 & $14.28 \%$ \\
\hline
\end{tabular}

\subsubsection{The Study of A-ECMS Based on $\lambda$ Maps with Driving Pattern Identification}

In this section, the recognized $\lambda$ map for each driving pattern is then implemented in the HEB model with the A-ECMS algorithm to evaluate the performance of this vehicle operated on other cycles. As reported in Chapter 6, the distance of the segment selected was found to significantly affect the accuracy of the predicted driving features. In this study, the distance of the segment is $45 \mathrm{~m}$.

Figure 7.10 compares the vehicle operation between A-ECMS based on $\lambda$ maps with the driving pattern identification and ECMSwDP simulation results over the Beijing cycle. The RDP identified in each segment is presented in Figure 7.10. The SOC trajectory observed with A-ECMS was comparable to that of the off-line ECMSwDP controller with the exception of the segment from $1320 \mathrm{~s}$ to $1800 \mathrm{~s}$. As shown in Table 7.4, the corrected fuel 
economy observed with A-ECMS with $\lambda$ maps from the RDP is $10.63 \mathrm{mpg}$, which is $4.83 \%$ gap to that observed using DP. The application of the A-ECMS improved the fuel economy by $50.78 \%$ (from 7.05 to $10.63 \mathrm{mpg}$ ) over the reference points observed with the rule-based control.

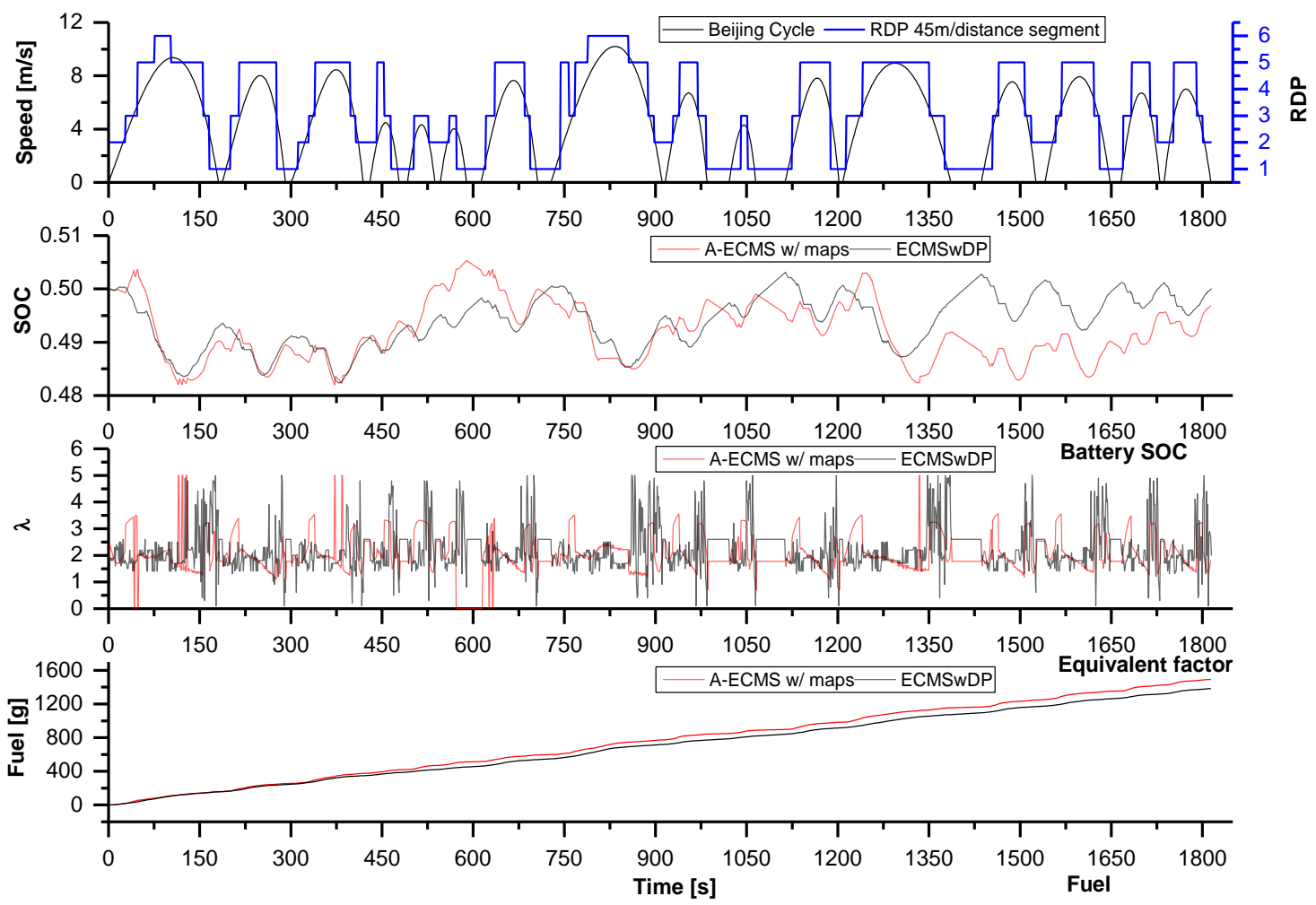

Figure 7.10 The comparison between A-ECMS based on $\lambda$ maps with the driving pattern identification and ECMSwDP simulation results over the Beijing cycle

Table 7.4 Simulation results of A-ECMS based on $\lambda$ maps with the driving pattern identification over the Beijing cycle

\begin{tabular}{|c|c|c|c|c|}
\hline & FE(mpg) & $\boldsymbol{\Delta S O C}$ & $\boldsymbol{F} \boldsymbol{E}_{\text {corr }}(\mathbf{m p g})$ & Gap to DP \\
\hline Rule-based & 7.05 & $-0.10 \%$ & 7.05 & $36.88 \%$ \\
\hline A-ECMS w/ $\boldsymbol{\lambda}(\boldsymbol{v}, \boldsymbol{a})$ maps & 10.73 & $-0.32 \%$ & 10.63 & $4.83 \%$ \\
\hline DP $(\boldsymbol{\alpha}=\mathbf{0}, \boldsymbol{\beta}=\mathbf{0}, \boldsymbol{\gamma}=\mathbf{0})$ & $\mathbf{1 1 . 1 7}$ & $\mathbf{0 . 0 0 \%}$ & $\mathbf{1 1 . 1 7}$ & $\mathbf{0 . 0 0 \%}$ \\
\hline
\end{tabular}

The potential of the A-ECMS with $\lambda$ maps of RDP based on the predicted driving features in improving the performance of a HEB is also examined using the WVU-CSI cycle as an example. As shown in Table 7.5, the corrected fuel economy observed with A-ECMS is $10.61 \%$ gap to that observed with DP. The application of A-ECMS with $\lambda$ maps of RDP 
based on the predicted driving features improved the fuel economy by $22.30 \%$ (from 8.61

to $10.53 \mathrm{mpg}$ ).

Table 7.5 Simulation results of A-ECMS based on $\lambda$ maps with the driving pattern identification over the WVU-CSI cycle

\begin{tabular}{|c|c|c|c|c|}
\hline & FE(mpg) & $\Delta$ SOC & $\boldsymbol{F E}$ corr $(\mathbf{m p g})$ & Gap to DP \\
\hline Rule-based & 8.74 & $-0.60 \%$ & 8.61 & $26.91 \%$ \\
\hline A-ECMS w/ $\boldsymbol{\lambda}$ maps & 10.65 & $-0.83 \%$ & 10.53 & $10.61 \%$ \\
\hline DP $(\boldsymbol{\alpha}=\mathbf{0}, \boldsymbol{\beta}=\mathbf{0}, \boldsymbol{\gamma}=\mathbf{0})$ & $\mathbf{1 1 . 7 8}$ & $\mathbf{0 . 0 0 \%}$ & $\mathbf{1 1 . 7 8}$ & $\mathbf{0 . 0 0 \%}$ \\
\hline
\end{tabular}

The simulation results of the Beijing cycle and WVU-CSI cycle demonstrated the potential of A-ECMS based on $\lambda$ maps with driving pattern identification in improving the fuel economy of a hybrid vehicle. It is evident that the A-ECMS with $\lambda$ maps can be considered as real-time sub-optimal controller.

\subsubsection{The A-ECMS with or without a Penalty Based on the Average of the $\lambda$ Extracted with Driving Pattern Identification}

Chapter 5 demonstrated that the average of the extracted $\lambda$ values derived using ECMSwDP was comparable to the optimal $\lambda$ value. The average extracted $\lambda$ value can be set as the constant $\lambda$ of the ECMS without a penalty function or the initial value $\lambda_{0}$ of the ECMS with a penalty function for a known driving condition or driving pattern. Table 7.6 shows the average $\lambda$ of the six RDPs derived using ECMSwDP.

Table 7.6 The average of the $\lambda$ values extracted corresponding to the six RDPs

\begin{tabular}{|c|c|c|c|c|c|c|}
\hline \multirow{2}{*}{ RDPs } & \multicolumn{9}{|c|}{ Pattern } \\
\cline { 2 - 7 } & $\mathbf{1}$ & $\mathbf{2}$ & $\mathbf{3}$ & $\mathbf{4}$ & $\mathbf{5}$ & $\mathbf{6}$ \\
\hline $\begin{array}{c}\text { Representative } \\
\text { Driving Cycle }\end{array}$ & $\begin{array}{c}\text { HHDDT } \\
\text { Creep }\end{array}$ & NYC BUS & NY Comp & $\begin{array}{c}\text { Commuter } \\
\text { No Init Idle }\end{array}$ & $\begin{array}{c}\text { HHDDT } \\
\text { Trans }\end{array}$ & Freeway \\
\hline $\begin{array}{c}\text { The average of the } \boldsymbol{\lambda} \\
\text { extracted }\left(\boldsymbol{\lambda}_{\text {ave }} \text { ) }\right.\end{array}$ & 1.831 & 1.694 & 1.636 & 2.188 & 2.125 & 2.026 \\
\hline
\end{tabular}

Figure 7.11 shows the structure of the proposed A-ECMS, where the adaptive equivalent factor is determined by the corresponding average of the $\lambda$ identified using the current and 
predicted driving pattern features. At each distance segment of a drive cycle, one $\lambda$ is available from one of the six average extracted $\lambda$, and based on the RDP.

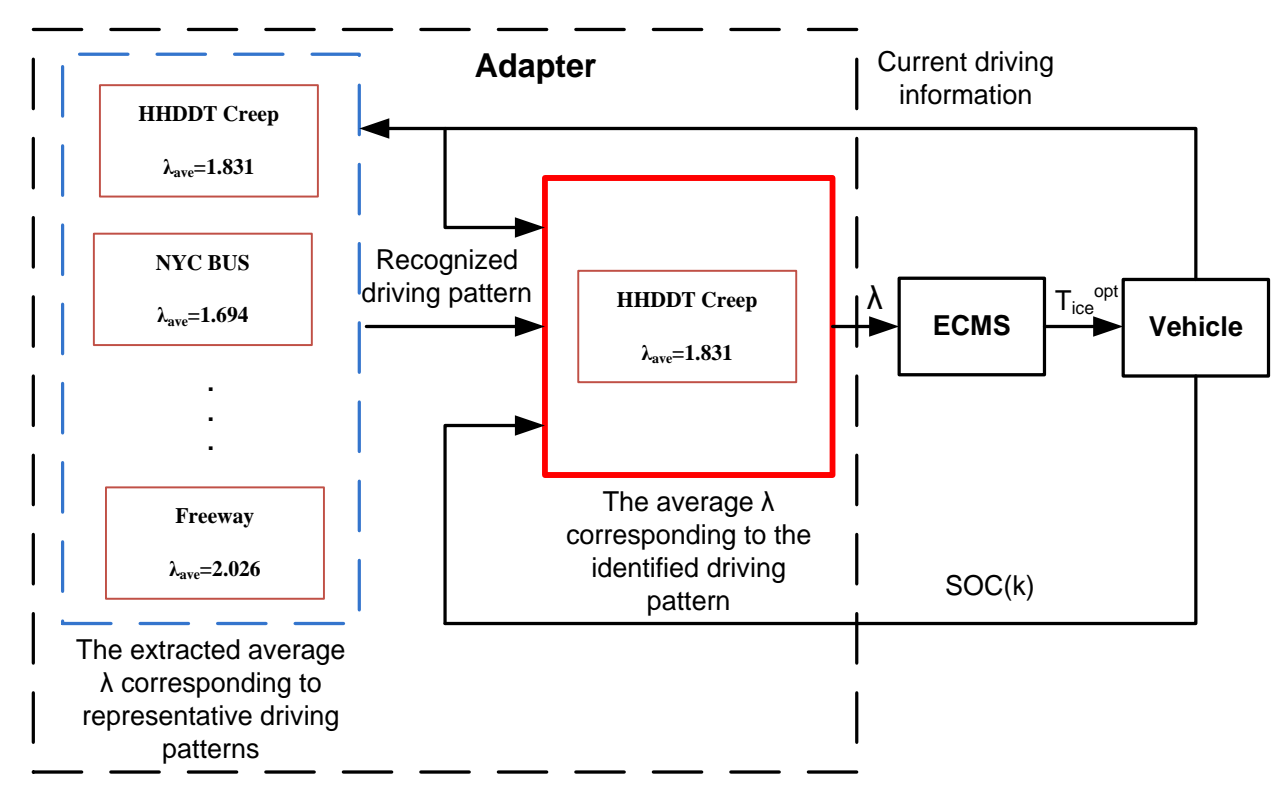

Figure 7.11 A-ECMS based on the average of the $\lambda$ values extracted with driving pattern identification

Figure 7.12 compares the operation of this vehicle between A-ECMS with or without a penalty based on the average of the $\lambda$ extracted with the driving pattern identification and ECMSwDP simulation results over the Beijing cycle. The SOC curves generated using the A-ECMS without a penalty deviated from the respective one generated by the off-line ECMSwDP controller. The battery was not sustainable. The SOC curves generated using the A-ECMS with a penalty function, however, adjusted the $\lambda$ according to the deviation between the feedback of the current SOC observed and the reference SOC. The SOC observed at the end of vehicle operation is very close to it's initial value. It is evident that the battery is charge sustainable when vehicle operates with A-ECMS with a penalty function. 


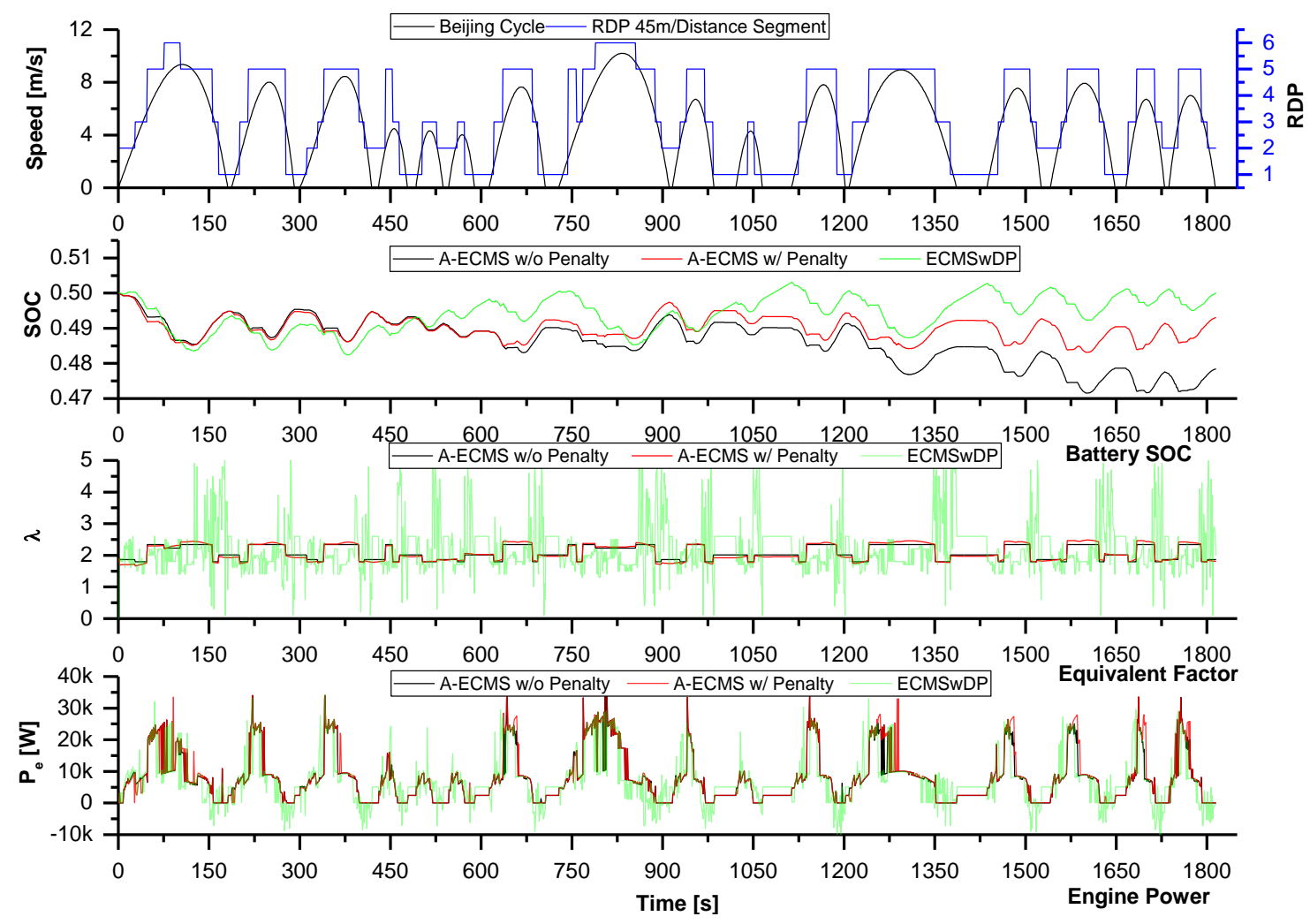

Figure 7.12 The comparison between A-ECMS with or without a penalty based on the average of the $\lambda$ extracted with the driving pattern identification and ECMSwDP simulation results over the Beijing cycle

Table 7.7 compares the fuel consumption observed with A-ECMS with or without a penalty based on the average of the $\lambda$ extracted with the driving pattern identification and ECMSwDP simulation. The corrected fuel economy observed with A-ECMS without the penalty function is $10.37 \mathrm{mpg}$, which is $7.16 \%$ gap to that observed with DP. The corrected fuel economy observed with A-ECMS with the penalty function is $10.55 \mathrm{mpg}$, which is $5.55 \%$ gap to that observed with DP. The fuel economy observed with and without the penalty function is comparable to that observed using DP. As shown in Table 7.7, the corrected fuel economy observed using A-ECMS with penalty function (10.55 mpg) is $49.65 \%$ better than that observed with the rule based control strategies $(7.05 \mathrm{mpg})$. 
Table 7.7 Simulation results by A-ECMS with or without a penalty based on the average of the $\lambda$ extracted with the driving pattern identification over the Beijing cycle

\begin{tabular}{|c|c|c|c|c|}
\hline & FE $(\mathbf{m p g})$ & $\Delta$ SOC & $\boldsymbol{F} E_{\text {corr }}(\mathbf{m p g})$ & Gap to DP \\
\hline Rule-based & 7.05 & $-0.10 \%$ & 7.05 & $36.88 \%$ \\
\hline A-ECMS w/o penalty & 10.09 & $-2.16 \%$ & 10.37 & $7.16 \%$ \\
\hline A-ECMS w/ penalty & 10.78 & $-0.7 \%$ & 10.55 & $5.55 \%$ \\
\hline DP $(\alpha=\beta=\gamma=0)$ & $\mathbf{1 1 . 1 7}$ & $\mathbf{0 . 0 0 \%}$ & $\mathbf{1 1 . 1 7}$ & $\mathbf{0 . 0 0 \%}$ \\
\hline
\end{tabular}

The potential of the A-ECMS with or without a penalty function based on the average of the $\lambda$ extracted with the driving pattern identification in improving the performance of the HEB is also examined using the WVU-CSI cycle as an example. As shown in Table 7.8, the corrected fuel economy observed with A-ECMS with a penalty function is $13.67 \%$ gap to that with DP. The corrected fuel economy observed using A-ECMS is $10.17 \mathrm{mpg}$, which is $18.12 \%$ higher than the $8.61 \mathrm{mpg}$ observed with the rule-based control strategy.

Table 7.8 Simulation results of A-ECMS with or without a penalty based on the average of the $\lambda$ extracted with the driving pattern identification over the WVU-CSI cycle

\begin{tabular}{|c|c|c|c|c|}
\hline & $\mathbf{F E}(\mathbf{m p g})$ & $\boldsymbol{\Delta S O C}$ & $\boldsymbol{F E}$ corr $(\mathbf{m p g})$ & Gap to DP \\
\hline Rule-based & 8.74 & $-0.60 \%$ & 8.61 & $26.91 \%$ \\
\hline A-ECMS w/o penalty & 10.59 & $-6.67 \%$ & 9.61 & $18.42 \%$ \\
\hline A-ECMS w/ penalty & 10.15 & $-0.24 \%$ & 10.17 & $13.67 \%$ \\
\hline $\mathbf{D P}(\boldsymbol{\alpha}=\boldsymbol{\beta}=\boldsymbol{\gamma}=\mathbf{0})$ & $\mathbf{1 1 . 7 8}$ & $\mathbf{0 . 0 0 \%}$ & $\mathbf{1 1 . 7 8}$ & $\mathbf{0 . 0 0 \%}$ \\
\hline
\end{tabular}

\subsubsection{The Evaluation of the Control Approaches Examined in This Research}

The extents of excellence of the controlling approaches were usually evaluated by comparing the corrected fuel economy observed using the controlling approaches developed with that obtained with DP. The closer the fuel economy obtained to that obtained with DP, the better this control algorithm was for approaching the optimal solution. In this research, the extent of excellence of a control approach in improving the fuel economy is further evaluated by examining its capability in achieving the potential to the best improvement (PTBI) solution using the corrected fuel economy observed with the 
reference control strategy and DP as the low and high limit, respectively. The PTBI in the corrected fuel economy $P T B I_{F E_{c o r r}}$ is defined as:

$$
P T B I_{F E_{\text {corr }}}=\frac{F E_{\operatorname{corr}(.)}-F E_{\text {corr }(\text { Rule-based })}}{F E_{D P}-F E_{\text {corr }(\text { Rule-based })}} \times 100 \quad \text { Equation } 7.1
$$

where $F E_{\operatorname{corr}(.)}$ is the corrected fuel economy achieved by the proposed control approaches; $F E_{D P}$ is the fuel economy obtained using DP (the preferred reference); and $F E_{\text {corr(Rule-based })}$ is the corrected fuel economy achieved using the rule-based controller (the bottom reference).

Table 7.9 summaries the corrected fuel economy and the $P T B I_{F E_{c o r r}}$ observed when this vehicle is operated on the Beijing cycle with controlling approaches examined in this research.

Table 7.9 Comparison of fuel economy and the change in SOC simulated using different control approaches over the Beijing cycle

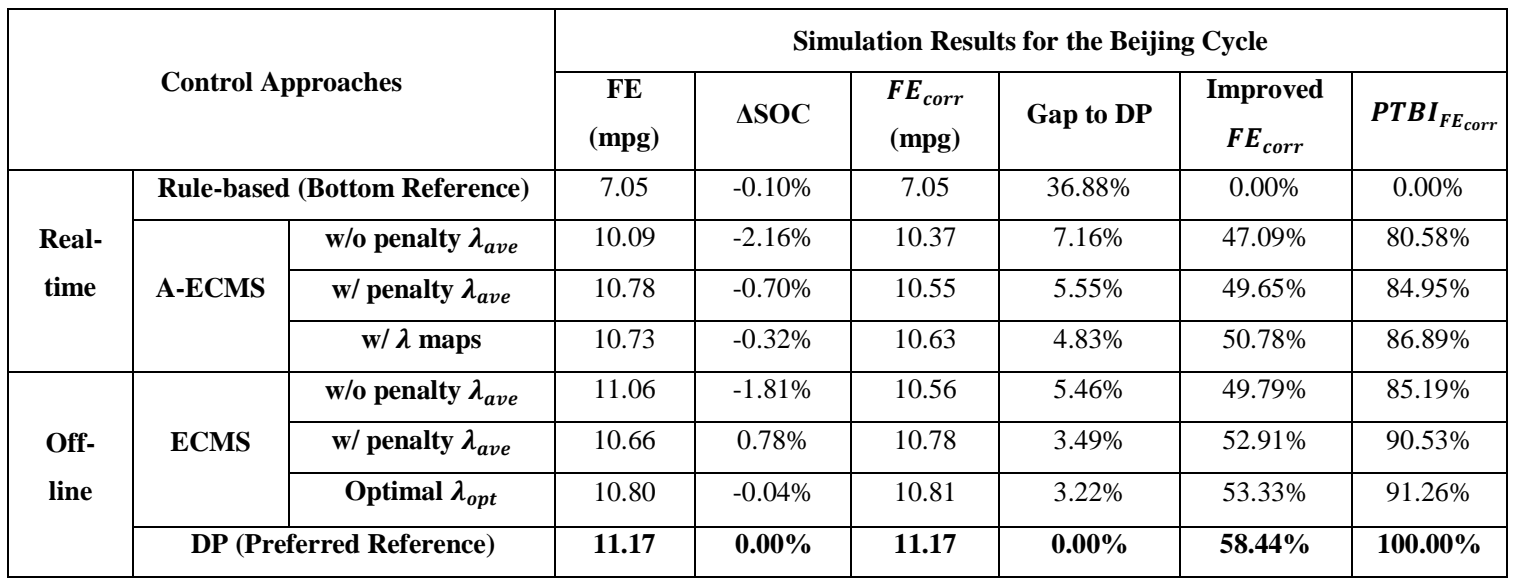

The gap to DP achieved with the A-ECMS with a penalty function and A-ECMS with $\lambda$ maps are $5.55 \%$ and $4.83 \%$, respectively. It is evident that the A-ECMS with a penalty function and A-ECMS with $\lambda$ maps can be considered as a sub-optimal controller capable of significantly improving the fuel economy of this hybrid vehicle. The $P T B I_{F E_{c o r r}}$ achieved with the A-ECMS with a penalty function (84.85\%) and A-ECMS with $\lambda$ maps 
(86.89\%) is slightly lower than the $P T B I_{F E_{c o r r}}$ achieved using the off-line ECMS with the selected $\lambda_{\text {ave }}$ and $\lambda_{\text {opt }}$ is $90.53 \%$ and $91.26 \%$, respectively.

Table 7.10 compares the fuel economy of this vehicle operated on the WVU-CSI cycle with real-time A-ECMS and predicted driving features and ECMS calculated off-line with a known driving cycle. The gap to DP achieved using A-ECMS with a penalty function and A-ECMS with $\lambda$ maps is $13.67 \%$ and $10.61 \%$, respectively. The application of the proposed A-ECMS with $\lambda$ maps of predicted RDPs improves the fuel economy from 8.61 mpg observed with the rule-based controller to $10.53 \mathrm{mpg}$.

Table 7.10 Comparison of fuel economy and the change in SOC simulated using different control approaches over the revised WVU-CSI cycle

\begin{tabular}{|c|c|c|c|c|c|c|c|c|}
\hline & \multirow{2}{*}{\multicolumn{2}{|c|}{ Control Approaches }} & \multicolumn{6}{|c|}{ Simulation Results for the WVU-CSI Cycle } \\
\hline & & & \multirow{2}{*}{$\begin{array}{c}\begin{array}{c}\text { FE } \\
(\mathbf{m p g})\end{array} \\
8.74\end{array}$} & \multirow{2}{*}{$\begin{array}{l}\text { SSOC } \\
-0.60 \%\end{array}$} & \multirow{2}{*}{ 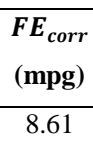 } & \multirow{2}{*}{$\begin{array}{l}\text { Gap to DP } \\
26.91 \%\end{array}$} & \multirow{2}{*}{$\begin{array}{c}\text { Improved } \\
\boldsymbol{F E}_{\text {corr }} \\
0.00 \%\end{array}$} & \multirow{2}{*}{ 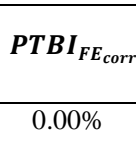 } \\
\hline \multirow{4}{*}{$\begin{array}{l}\text { Real- } \\
\text { time }\end{array}$} & \multicolumn{2}{|c|}{ Rule-based (Bottom Reference) } & & & & & & \\
\hline & \multirow{3}{*}{ A-ECMS } & w/o penalty $\lambda_{\text {ave }}$ & 10.59 & $-6.67 \%$ & 9.61 & $18.42 \%$ & $11.61 \%$ & $31.55 \%$ \\
\hline & & w/ penalty $\lambda_{\text {ave }}$ & 10.15 & $-0.24 \%$ & 10.17 & $13.67 \%$ & $18.12 \%$ & $49.21 \%$ \\
\hline & & w/ $\lambda$ maps & 10.65 & $-0.83 \%$ & 10.53 & $10.61 \%$ & $22.30 \%$ & $60.57 \%$ \\
\hline \multirow{4}{*}{ Off-line } & \multirow{3}{*}{ ECMS } & w/o penalty $\lambda_{\text {ave }}$ & 11.89 & $-9.67 \%$ & 10.24 & $13.07 \%$ & $18.93 \%$ & $51.42 \%$ \\
\hline & & w/ penalty $\lambda_{\text {ave }}$ & 10.65 & $0.17 \%$ & 10.66 & $9.51 \%$ & $23.81 \%$ & $64.67 \%$ \\
\hline & & Optimal $\lambda_{\text {opt }}$ & 11.10 & $-0.09 \%$ & 11.09 & $5.86 \%$ & $28.80 \%$ & $78.23 \%$ \\
\hline & \multicolumn{2}{|c|}{ DP (Preferred Reference) } & 11.78 & $0.00 \%$ & 11.78 & $0.00 \%$ & $36.82 \%$ & $100.00 \%$ \\
\hline
\end{tabular}

\subsection{Simulation with a Real-World Route}

The potential of the proposed A-ECMS control strategies in improving the fuel economy of this hybrid bus is also examined over the actual transit bus data sampled in Route 6 in SEAT district in Preston, CT. The contribution of Pingen Chen and the technical staff associated with the testing campaigns should be acknowledged. Figure 7.13 and Figure 7.14 show the route of the transit bus and the actual vehicle speed data sampled in SEAT. 


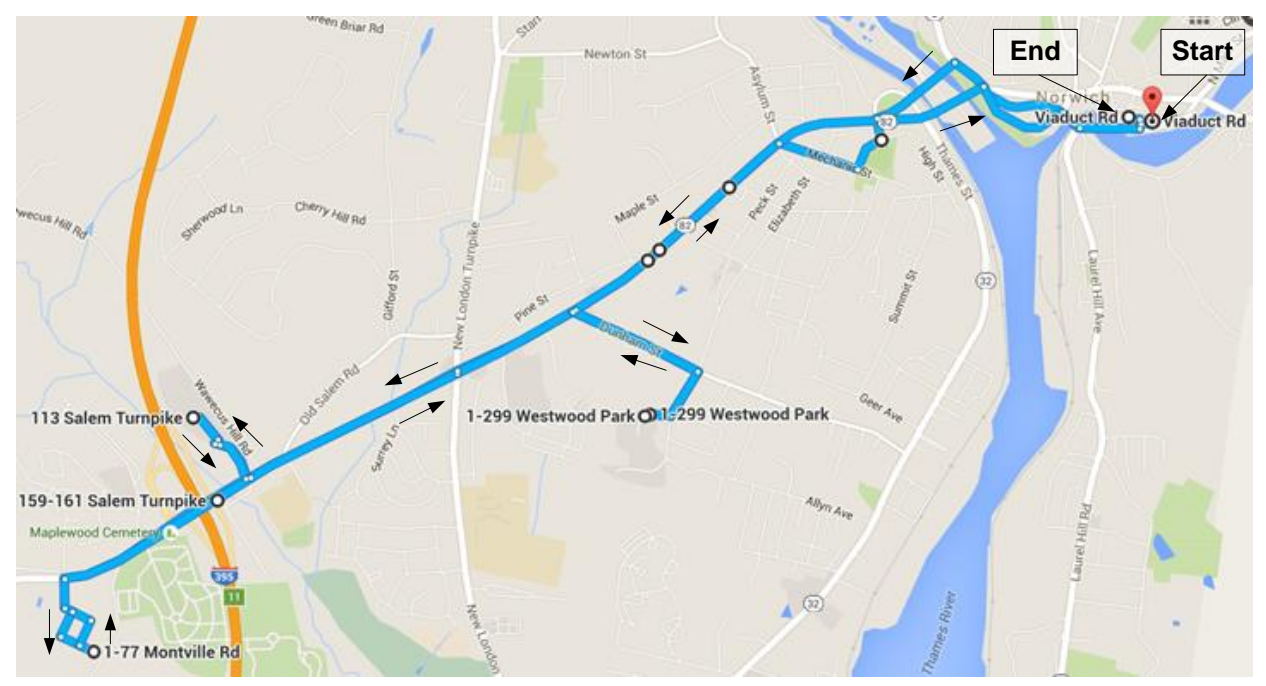

Figure 7.13 Transit bus Route 6 in the SEAT district of Preston, CT

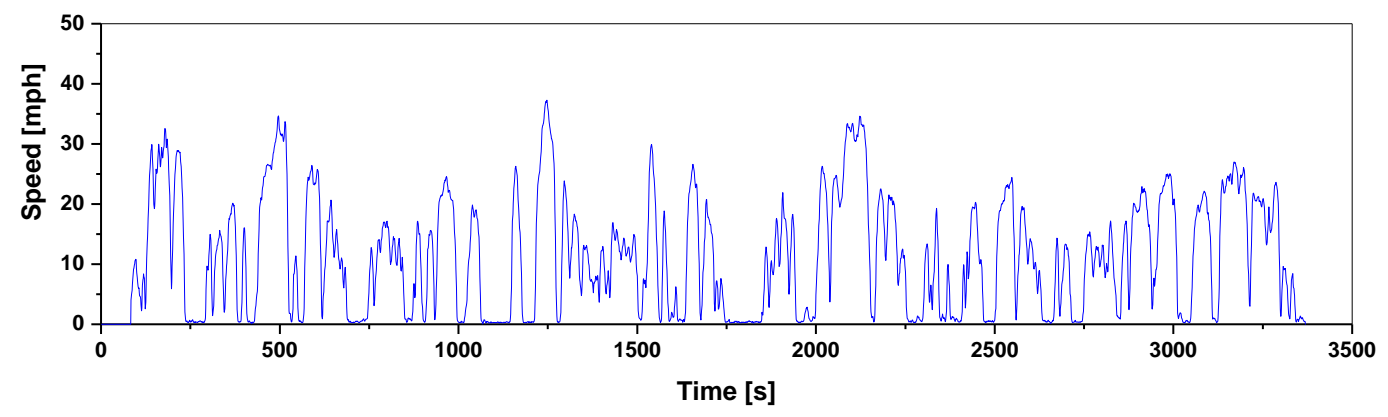

Figure 7.14 The actual Rt6 cycle sampled in SEAT

This route consisted of three different road types: inner-city traffic fearturing an average speed below $10 \mathrm{mph}$, an urban route with a speed limit of $30 \mathrm{mph}$, and a suburban route with relatively long distances between stops and the speed limit was $50 \mathrm{mph}$. There were several traffic lights and stop signs in this route. These features, along with the presence of flagmen, characterized the operation of the transit bus on this route as having frequent stops and goes. The total distance of round trip was 10.5 miles.

The effect of the proposed A-ECMS approaches on the fuel economy of the hybrid bus operated over route 6 in SEAT was examined. Figure 7.15 compares the performance of this hybrid vehicle with the rule-based controller, the proposed A-ECMS with a penalty function, the A-ECMS with $\lambda$ maps of the identified RDPs, and the DP algorithem when 
this HEV is operated over the actural route data shown in Figure 7.14. The driving patterns are identified based on the assumed segment distance of $45 \mathrm{~m}$ and shown in Figure 7.15. The SOC observed at the end of this actual cycle is comparable to the initial one. The sustainable operation of the battery is satisfied although the variation in SOC are significantly different. As shown in Figure 7.15, the fluctuation of the SOC trajectory observed with the A-ECMS with a penalty function is much smoother than that observed with the A-ECMS with $\lambda$ maps and DP. This is due to the contribution of the SOC penalty function, which forces the system to charge or discharge the battery when the SOC is away from the reference value regardless the operating conditions of the vehicle. The variation of the SOC observed with A-ECMS with $\lambda$ maps is comparable to that observed with the DP algorithm although the $\lambda$ value at each point may be siginificantly different.

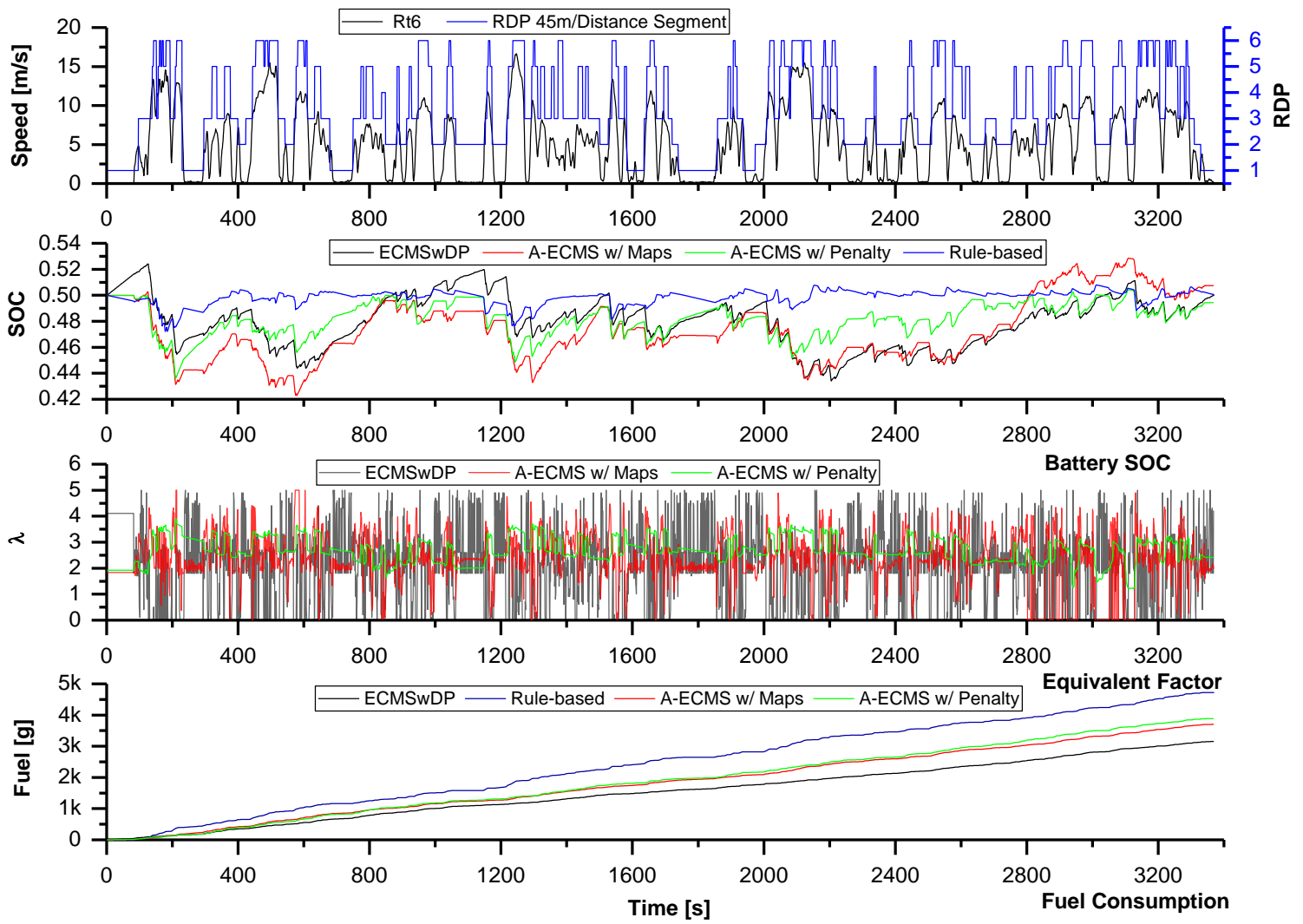

Figure 7.15 The comparison of simulation results from the proposed A-ECMS based on $\lambda$ maps of the predicted RDPs, the A-ECMS with a penalty based on the average of the $\lambda$ extracted with driving pattern identification, rule-based, and DP over Rt6 in SEAT 
Table 7.11 summarizes the simultated fuel economy, the gap to DP, and the change in SOC when this vehicle is operated over Rt6. The corrected fuel economy achieved with the AECMS with a penalty function and the A-ECMS with $\lambda$ maps is $8.46 \mathrm{mpg}$ and $8.97 \mathrm{mpg}$, which is $80.80 \%$ and $85.67 \%$ of that observed with DP, respectively. When compared with the fuel economy $(6.19 \mathrm{mpg})$ observed with the rule-based control strategy, the application of the A-ECMS with $\lambda$ maps improved the fuel economy by $44.91 \%$ (from 6.19 to 8.97 mpg). The gap to DP achieved with the A-ECMS with a penalty function and A-ECMS with $\lambda$ maps is $19.19 \%$ and $14.33 \%$, respectively. It is evident that the A-ECMS control approaches proposed in this research are able to significantly improve the fuel economy when this vehicle is operated over an actual route.

Table 7.11 Comparison of fuel economy and the change in SOC for different control approaches over the actual Rt6 operational data

\begin{tabular}{|c|c|c|c|c|c|c|}
\hline & $\begin{array}{c}\text { FE } \\
(\mathbf{m p g})\end{array}$ & $\Delta \mathbf{S O C}$ & $\begin{array}{c}\boldsymbol{F} E_{\text {corr }} \\
(\mathbf{m p g})\end{array}$ & Gap to DP & $\begin{array}{c}\text { Improved } \\
\boldsymbol{F} E_{\text {corr }}\end{array}$ & $\boldsymbol{P T B I}_{\boldsymbol{F E}}$ \\
\hline Rule-based & 6.19 & $0.05 \%$ & 6.19 & $40.88 \%$ & $0.00 \%$ & $0.00 \%$ \\
\hline A-ECMS w/ penalty & 8.51 & $-0.56 \%$ & 8.46 & $19.19 \%$ & $36.67 \%$ & $53.04 \%$ \\
\hline A-ECMS w/ $\boldsymbol{\lambda}$ maps & 8.90 & $1.49 \%$ & 8.97 & $14.33 \%$ & $44.91 \%$ & $64.95 \%$ \\
\hline $\mathbf{D P}(\boldsymbol{\alpha}=\boldsymbol{\beta}=\boldsymbol{\gamma}=\mathbf{0})$ & $\mathbf{1 0 . 4 7}$ & $\mathbf{0 . 0 0 \%}$ & $\mathbf{1 0 . 4 7}$ & $\mathbf{0 . 0 0 \%}$ & $\mathbf{6 9 . 1 4 \%}$ & $\mathbf{1 0 0 . 0 0 \%}$ \\
\hline
\end{tabular}

\subsection{Summary}

The A-ECMS model package developed in this research consists of a driving feature prediction model, a vehicle operation pattern identification model, and an A-ECMS model capable of adjusting the $\lambda$, a parameter deciding the ratio of power provided by the ICE and battery of the hybrid vehicle.

This chapter explored the performance of this hybrid vehicle operated with ECMS, DP, AECMS with and without a penalty function, and A-ECMS with $\lambda$ maps of RDPs using the Beijing and WVU-CSI cycles as examples. The simulation results demonstrated the capability of the proposed A-ECMS with $\lambda$ maps and the upcoming vehicle operation 
features prediction model in improving the corrected fuel economy while satisfying the charging sustainability of the battery. The corrected fuel economy observed with A-ECMS with penalty function was $5.55 \%$ and $13.67 \%$ gap to that observed with DP when operated over the Beijing cycle and WVU-CSI cycle, respectively.

The proposed model package is also applied to optimize the real-time operation of this hybrid vehicle using actual transit bus operation data sampled in SEAT. The corrected fuel economy observed using A-ECMS with $\lambda$ maps over the actual operation data is $14.33 \%$ gap to that observed with DP simulation results.

A parameter noted as the potential to the best improvement (PTBI) was proposed as a criterion for evaluating the extent of excellence of the real-time controllers (A-ECMS with and without a penalty function, and $\lambda$ maps) and off-line controllers (DP, ECMS with and without a penalty function, and ECMS with the optimal $\lambda$ ). The $P T B I_{F E_{c o r r}}$ of the AECMS with $\lambda$ maps observed was $86.89 \%, 60.57 \%$, and $64.95 \%$ compared to rule-based simulation results when operated over the Beijing cycle, WVU-CSI cycle, and actual bus operation data sampled in SEAT, respectively.

It is evident that the proposed A-ECMS model is able to significantly improve the fuel economy while maintaining the sustainable charging of the battery. 


\section{Conclusion and Future Work}

\subsection{Conclusion}

This dissertation presents the development of the methodology capable of developing the real-time optimal control strategies of HEBs, their application in improving the fuel economy of a parallel hybrid transit bus operated over different cycles, and the evaluation of the extent of excellence of proposed approaches in improving the fuel economy of this vehicle.

The following are the main contributions of this research:

1) The A-ECMS model package developed in this research consists of a driving pattern feature prediction model capable of predicting the vehicle operation features with the history and current driving information, a driving pattern identification model capable of identifying the representative driving patterns, and an A-ECMS model capable of the adjusting the $\lambda$, a parameter deciding the ratio of power provided by the ICE and battery of the hybrid vehicle.

(2) The combination of the ECMS with DP proposed in this research is able to generate the $\lambda$ maps and near optimal $\lambda$ of the driving cycles. The $\lambda$ maps of 6 RDPs as a function of the velocity and acceleration rate were generated using ECMSwDP. At each distance segment of a drive cycle, the $\lambda$ value is available from one of the six $\lambda$ maps based on the predicted driving patterns and utilized in a real-time A-ECMS controller.

(3) The codes for the optimized control of a parallel hybrid transit bus with off-line approaches (DP, ECMS, ECMSwDP), and real-time approaches (A-ECMS, and rule based control strategy) have been developed. The effects of the control strategies developed using the real-time and off-line approaches on the fuel economy, changes in SOC of the battery, 
and variation in $\lambda$ were numerically simulated using the Beijing cycle, WVU-CSI cycle, and an actual route sampled in SEAT.

(4) A parameter noted as the Hybrid Energy Recovery Potential Index was proposed to evaluate the ratio of the braking energy recoverable relative to the propulsion energy. This parameter can be used to evaluate the potential energy recovery benefit for a specific route to be served with hybrid vehicles. In this research, the HERPI was used as the response variable of stepwise regression in identifying the key driving pattern features representing the operation of a hybrid vehicle.

(5) A parameter noted as the potential to the best improvement was defined. In this research, the PTBI is used as a criterion in evaluating the extent of excellence of the controlling approaches in improving the fuel economy of a hybrid vehicle. The fuel economy observed using DP and a rule based controller were defined as the upper limit and lower limit, respectively, in evaluating the extent of excellence of control strategies developed in this research.

Based on the simulation results, the following conclusions can be drawn:

(1) The optimization of hybrid vehicle operation using DP provides the globally best solution. The fuel economy observed using DP was defined as the upper limit in evaluating the extent of excellence of control strategies developed in this research. The optimization of this vehicle's operation with the Beijing cycle and WVU-CSI cycle using DP improved the fuel economy by $58.44 \%$ and $34.02 \%$ compared to rule-based simulation results.

(2) The average $\lambda$ value extracted using ECMSwDP was comparable to the optimal $\lambda$ value of ECMS. Accordingly, the average $\lambda$ value extracted using ECMSwDP can be considered as the initial $\lambda$ value $\left(\lambda_{0}\right)$ for the application of ECMS with a penalty function. The corrected fuel economy observed using ECMS with a penalty function $\left(\lambda_{0}\right)$ was only $3.49 \%$ (Beijing cycle) and 9.51\% (WVU-CSI cycle) gap to that observed using DP. 
(3) The HERPI is dominated by the average velocity and average acceleration rate, and is expressed as HERPI $=0.302+1.792 \times \bar{\alpha}_{+}-0.016 \times \bar{v}$ for the hybrid vehicle simulated in this research.

(4) After the K-means cluster analysis, six standard cycles (NYC Bus, Freeway, NY Comp, Commuter No Init Idle, HHDDT Creep, and HHDDT Trans) were selected as the RDPs out of the 54 driving cycles examined.

(5) The control strategies developed using A-ECMS were able to significantly improve the fuel economy while maintaining the charging sustainability of the battery. The corrected fuel economy observed with A-ECMS with a penalty function and RDPs was $5.55 \%$, $13.67 \%$, and $19.19 \%$ gap to that observed with DP when operated over the Beijing cycle and WVU-CSI cycle, and the actual transit bus route, respectively. The corrected fuel economy observed with A-ECMS with $\lambda$ maps of the RDPs was $4.83 \%, 10.61 \%$, and $14.33 \%$ gap to that observed with DP when operated on the Beijing cycle, WVU-CSI cycle, and actual transit bus route, respectively.

(6) The real-time control strategies developed using A-ECMS were suboptimal compared to optimal one developed using DP. The observed $P T B I_{F E_{c o r r}}$ of the A-ECMS with a penalty function and RDPs were 84.95, 49.21, and 53.04\% compared to rule-based simulation results when this hybrid bus was operated on the Beijing cycle, WVU-CSI cycle, and actual transit bus route, respectively. The observed $P T B I_{F E_{c o r r}}$ of the A-ECMS with $\lambda$ maps of the RDPs observed were $86.89 \%, 60.57 \%$, and $64.95 \%$ compared to rule-based simulation results, accordingly.

\subsection{Future Work}

The real time control strategies developed using the A-ECMS approaches were suboptimal compared to those developed using DP. The performance of A-ECMS in improving the 
fuel economy of a hybrid vehicle can be further improved if the following works can be completed:

(1) The development of the representative driving patterns of transit buses operated on a fixed route. This improvement will provide the $\lambda$ map of the RDPs representing the actual cycle and make it possible for A-ECMS to achieve better fuel economy toward that of DP. (2) The current model was evaluated using the fuel economy as reference. Such a model can be further improved to include $\mathrm{CO}_{2}$ and greenhouse gas emissions and emissions of pollutants such as PM and NOx. The ECMS concepts developed in this research can be further extended to the optimization of hybrid vehicle operation regarding the emissions of GHG and pollutants.

(3) The current model was evaluated using a parallel hybrid bus as a platform. It can be further applied to plug-in hybrid vehicles. The application of the A-ECMS in the plug-in hybrid vehicle can further minimize the fuel consumed by the ICEs and exhaust emissions in a specific area while operated on a specific route for a specific service. 


\section{Bibliography}

Ambuhl, Daniel, and Lino Guzzella. 2009. "Predictive reference signal generator for hybrid electric vehicles." IEEE Transactions on Vehicular Technology 58, no. 9 (2009): 4730-4740.

Amiri, Meisam, Mohsen Esfahanian, Mohammad Reza Hairi-Yazdi, and Vahid Esfahanian. 2009. "Minimization of power losses in hybrid electric vehicles in view of the prolonging of battery life." Journal of Power Sources 190, no. 2 (2009): 372-379.

Bayindir, Kamil Çağatay, Mehmet Ali Gözüküçük, and Ahmet Teke. 2011. "A comprehensive overview of hybrid electric vehicle: powertrain configurations, powertrain control techniques and electronic control units." Energy Conversion and Management 52, no. 2 (2011): 1305-1313.

Bilbao, Endika, Barrade, Philippe, Ion Etxeberria-Otadui, Alfred Rufer, Sergio Luri, and Inigo Gil. 2014. "Optimal energy management strategy of an improved elevator with energy storage capacity based on dynamic programming." Industry Applications, IEEE Transactions 50, no. 2 (2014): 1233-1244.

Cassebaum, O., and B. Bäker. 2011. "Predictive supervisory control strategy for parallel HEVs using former velocity trajectories." In 2011 IEEE Vehicle Power and Propulsion Conference. pp. 1-6. IEEE, 2011.

Chan, Chingchuen. 2002. "The state of the art of electric and hybrid vehicles." Proceedings of the IEEE 90, no. 2 (2002): 247-275.

Chan, Kit Yan, Tharam S. Dillon, and Elizabeth Chang. 2013. "An intelligent particle swarm optimization for short-term traffic flow forecasting using on-road sensor systems." IEEE Transactions on Industrial Electronics 60, no. 10 (2013): 47144725. 
Chan, Kit Yan, Tharam S. Dillon, Jaipal Singh, and Elizabeth Chang. 2012. "Neuralnetwork-based models for short-term traffic flow forecasting using a hybrid exponential smoothing and Levenberg-Marquardt algorithm." Intelligent Transportation Systems, IEEE Transactions 13, no. 2 (2012): 644-654.

Chen, Pingen. 2010. Optimization of a heavy-duty hybrid transit bus operated over transient cycles. West Virginia University, 2010.

Clark, Nigel N., Feng Zhen, and W. Scott Wayne. 2009. Assessment of hybrid-electric transit bus technology. Transportation Research Board, Washington, DC, 2009.

County, King. 2005. New york city transit drives hybrid electric buses into the future. National Renewable Energy Laboratory (NREL), Golden, CO , 2005.

De Jager, Bram, Maarten Steinbuch, and Thijs van Keulen. 2008. "An adaptive sub-optimal energy management strategy for hybrid drive-trains." In IFAC Proceedings. 41, no. 2 (2008): 102-107.

Delprat, Sebastien, Thierry-Marie Guerra, and Janette Rimaux. 2002. "Optimal control of a parallel powertrain: from global optimization to real time control strategy." In Vehicular Technology Conference. pp. 2082-2088. IEEE, 2002.

Ehsani, Mehrdad, Yimin Gao, and Karen L. Butler. 1999. "Application of electrically peaking hybrid (ELPH) propulsion system to a full-size passenger car with simulated design verification." Vehicular Technology, IEEE Transactions 48(6), 1779-1787.

Eick, Steven. 2012. "Heavy duty diesel truck and bus hybrid powertrain study." Select Engineering Services, Layton UT, 2012.

Ericsson, Eva. 2011. "Independent driving pattern factors and their influence on fuel-use and exhaust emission factors." Research Part D: Transport and Environment 6, no. 5 (2001): 325-345. 
Falamarzi, Yashar, Narges Palizdan, Yuk Feng Huang, and Teang Shui Lee. 2014. "Estimating evapotranspiration from temperature and wind speed data using artificial and wavelet neural networks (WNNs)." Agricultural Water Management 140 (2014): 26-36.

Hajimiri, M. H., and F. R. Salmasi. 2006. "A fuzzy energy management strategy for series hybrid electric vehicle with predictive control and durability extension of the battery." In 2006 IEEE Conference on Electric and Hybrid Vehicles. pp. 1-5. IEEE, 2006.

Han, Jihun, Youngjin Park, and Dongsuk Kum. 2014. "Optimal adaptation of equivalent factor of equivalent consumption minimization strategy for fuel cell hybrid electric vehicles under active state inequality constraints." Journal of Power Sources 267 (2014): 491-502.

He, Hongwen, Chao Sun, and Xiaowei Zhang. 2012. "A method for identification of driving patterns in hybrid electric vehicles based on a LVQ neural network." Energies 5 no. 9 (2012): 3363-3380.

Hinman, R. S. 2006. "SPSS for psychologists." Palgrave Macmillan, Australia, 2006.

Jairo A., Sandoval Leon. 2011. Study of transit bus duty cycle and its influence on fuel economy and emissions of diesel-electric hybrids. West Virginia University, 2011.

Johnson, Valerie H., Keith B. Wipke, and David J. Rausen. 2000. "HEV control strategy for realtime optimization of fuel economy and emissions." SAE Transactions 109, no. 3 (2000): 1677-1690.

Jolliffe, Ian. 2002. Principal component analysis. John Wiley \& Sons, Ltd, 2002.

Khan, Abm Siddiqur Rahman. 2009. Route and grade sensitive modeling of fuel efficiency and emissions for diesel buses. West Virginia University, 2009. 
Kim, Namwook, Sukwon Cha, and Huei Peng. 2011. "Optimal control of hybrid electric vehicles based on Pontryagin's minimum principle." IEEE Transactions on Control Systems Technology 19, no. 5 (2011): 1279-1287.

Kirk, Donald E. 2012. Optimal control theory: an introduction. Courier Corporation, 2012.

Kutter, Steffen, and Bernard Bäker. 2010. "Predictive online control for hybrids: resolving the conflict between global optimality, robustness and real-time capability." In 2010 IEEE Vehicle Power and Propulsion Conference. pp. 1-7. IEEE, 2010.

Larsson, Viktor, Lars Johannesson Mårdh, Bo Egardt, and Sten Karlsson. 2014. "Commuter route optimized energy management of hybrid electric vehicles." IEEE Transactions on Intelligent Transportation Systems 15, no. 3 (2014): 1145-1154.

Lee, Hyeoun-Dong, Euh-Suh Koo, Seung-Ki Sul, Joohn-Sheok Kim, M. Kamiya, H. Ikeda, S. Shinohara, and H. Yoshida. 2000. "Torque control strategy for a parallel hybrid vehicle using fuzzy logic." IEEE Industry Applications Magazine 6, no. 6 (2000): $33-38$.

Lee, Seungjae, Young-Ihn Lee, and Bumcheol Cho. 2006. "Short-Term travel speed prediction models in car navigation systems." Journal of Advanced Transportation 40, no. 2 (2006): 122-139.

Lee, Seungjae, Young-Ihn Lee, and Bumcheol Cho. 2006. "Short-Term travel speed prediction models in car navigation systems." Journal of Advanced Transportation 40, no. 2 (2006): 122-139.

Lin, Chan-Chiao, Huei Peng, Soonil Jeon, and Jang Moo Lee. 2002. "Control of a hybrid electric truck based on driving pattern recognition." In Proceedings of the 2002 Advanced Vehicle Control Conference. Hiroshima, Japan. 2002. 
Lin, Chan-Chiao, Soonil Jeon, Huei Peng, and Jang Moo Lee. 2004. "Driving pattern recognition for control of hybrid electric trucks." Vehicle System Dynamics 42, no. 1-2 (2004): 41-58.

Lin, Chan-Chiao, Soonil Jeon, Huei Peng, and Jang Moo Lee. 2004. "Driving pattern recognition for control of hybrid electric trucks." Vehicle System Dynamics 42, no. 1-2 (2004): 41-58.

Mansour, Charbel, and Denis Clodic. 2012. "Optimized energy management control for the Toyota hybrid system using dynamic programming on a predicted route with short computation time." International Journal of Automotive Technology 13, no. 2 (2012): 309-324.

Mao, Xiaojian, Hu Zhong, Guoqiang Ao, Junxi Wang, Hangbo Tang, Jing Feng, and Bin Zhuo. 2008. "Development and performance validation of an ISG diesel hybrid power-train for city transit buses - part I: parameter matching and system." In 2008 IEEE Vehicle Power and Propulsion Conference. pp. 1-6. IEEE, 2008.

Marano, V., G. Rizzoni, P. Tulpule, Q. Gong, and H. Khayyam. 2012. "Intelligent energy management for plug-in hybrid electric vehicles: the role of ITS infrastructure in vehicle electrification." Oil \& Gas Science and Technology-Revue d'IFP Energies Nouvelles 67, no. 4 (2012): 575-587.

Markel, Tony. 2006. "Plug-In HEV vehicle design options and expectations." In Proceedings of the ZEV Technology Symposium California Air Resources Board. vol. 27, Sacramento, CA, USA, 2006.

Miller, Scott. $\quad 2011 . \quad$ "Traffic $\quad$ Modeling." http://home2.fvcc.edu/ dhicketh/DiffEqns/Spring11projects/Scott_Miller/Project. $p d f$. 
Montazeri-Gh, M., A. Fotouhi, and A. Naderpour. 2011. "Driving patterns clustering based on driving features analysis." Proceedings of the Institution of Mechanical Engineers, Part C: Journal of Mechanical Engineering Science 225, no. 6 (2011): 1301-1317.

Montazeri-Gh, Morteza, Amir Poursamad, and Babak Ghalichi. 2006. "Application of genetic algorithm for optimization of control strategy in parallel hybrid electric vehicles." Journal of the Franklin Institute 343, no. 4 (2006): 420-435.

Murphey, Yi L., Zhihang Chan, Leo Kiliaris, Jungme Park, Ming Kuang, Abul Masrur, and Anthony Phillips. 2008. "Neural learning of predicting driving environment." Army Tank Automotive Research Development and Engineering Center, Warren MI, 2008.

Musardo, Cristian, Giorgio Rizzoni, Yann Guezennec, and Benedetto Staccia. 2005. "AECMS: an adaptive algorithm for hybrid electric vehicle energy management." European Journal of Control 11, no. 4 (2005): 509-524.

Onori, Simona, and Lorenzo Serrao. 2011. "On Adaptive-ECMS strategies for hybrid electric vehicles." In Proceedings of the International Scientific Conference on Hybrid and Electric Vehicles. pp. 6-7, Malmaison, France, 2011.

Onori, Simona, Lorenzo Serrao, and Giorgio Rizzoni. 2010. "Adaptive equivalent consumption minimization strategy for hybrid electric vehicles." In ASME 2010 Dynamic Systems and Control Conference. pp. 499-505. American Society of Mechanical Engineers, 2010.

Paganelli, Gino, Sebastien Delprat, Thierry-Marie Guerra, Janette Rimaux, and JeanJacques Santin. 2002. "Equivalent consumption minimization strategy for parallel hybrid powertrains." In Vehicular Technology Conference, 2002. VTC Spring 2002. IEEE 55th. vol. 4, pp. 2076-2081, IEEE, 2002. 
Panday, Aishwarya, and Hari Om Bansal. 2014. "A review of optimal energy management strategies for hybrid electric vehicle." International Journal of Vehicular Technology 18 (2014):1-19.

Park, Jungme, Dai Li, Yi L. Murphey, Johannes Kristinsson, Ryan McGee, Ming Kuang, and Tony Phillips. 2011. "Real time vehicle speed prediction using a neural network traffic model." In Neural Networks (IJCNN), The 2011 International Joint Conference. pp. 2991-2996, IEEE, 2011.

Pisu, Pierluigi, and Giorgio Rizzoni. 2007. "A comparative study of supervisory control strategies for hybrid electric vehicles." IEEE Transactions on Control Systems Technology 15, no. 3 (2007): 506-518.

Rajagopalan, Arun, Gregory Washington, Giorgio Rizzoni, and Yann Guezennec. 2003. Development of fuzzy logic and neural network control and advanced emissions modeling for parallel hybrid vehicles. Center for Automotive Research, Intelligent Structures and Systems Laboratory, Columbus, Ohio, 2003.

Rajamani, Rajesh. 2011. Vehicle dynamics and control. Springer Science \& Business Media, 2011.

Rousseau, Aymeric, Sylvain Saglini, Michael Jakov, Donald Gray, and Keith Hardy. 2002. Trade-offs between fuel economy and NOx emissions using fuzzy logic control. No. ANL/ES/CP-108487. Argonne National Lab., IL, 2002.

Salmasi, Farzad Rajaei. 2007. "Control strategies for hybrid electric vehicles: Evolution, classification, comparison, and future trends." IEEE Transactions on Vehicular Technology 56, no. 5 (2007): 2393-2404.

Schouten, Niels J., Mutasim A. Salman, and Naim A. Kheir. 2002. "Fuzzy logic control for parallel hybrid vehicles." IEEE Transactions on Control Systems Technology 10, no. 3 (2002): 460-468. 
Sharer, Phillip, Romain Leydier, and Aymeric Rousseau. 2007. "Impact of drive cycle aggressiveness and speed on HEVs fuel consumption sensitivity." SAE Paper, No. 2007-01-0281.

Shen, Luou. 2008. Freeway travel time estimation and prediction using dynamic neural networks. Florida International University, 2008.

Slezak, Lee. 2013. Autonomie Training Part 1 Overview. http://www.autonomie.net/docs/7\%20-\%20Training/training_part1.pdf.

Smith, Lindsay I. 2002. "A tutorial on Principal Components Analysis." http://www.cs.otago.ac.nz/cosc453/student_tutorials/principal_components.pdf.

Sorrentino, Marco, Gianfranco Rizzo, and Ivan Arsie. 2011. "Analysis of a rule-based control strategy for on-board energy management of series hybrid vehicles." Control Engineering Practice 19, no. 12 (2011): 1433-1441.

Sundstrom, Olle, and Lino Guzzella. 2009. "A generic dynamic programming Matlab function." In 2009 IEEE Control Applications,(CCA) \& Intelligent Control, (ISIC). pp. 1625-1630, IEEE, 2009.

Tie, Siang Fui, and Chee Wei Tan. 2013. "A review of energy sources and energy management system in electric vehicles." Renewable and Sustainable Energy Reviews 20, (2013): 82-102.

U.S. Department of Energy, Energy Information Administration. 2014. National transportation statistics. June 5. 2014, http://www.eia.doe.gov/mer/consump.html.

Van Lint, Hans., and Chris Van Hinsbergen. 2012. "Short-Term traffic and travel time prediction models." Artificial Intelligence Applications to Critical Transportation (Artificial Intelligence Applications to Critical Transportation Issues 22 (2012): 2241) 22 (2012): 22-41. 
Vlahogianni, Eleni I., John C. Golias, and Matthew G. Karlaftis. 2004. "Short-term traffic forecasting: overview of objectives and methods." Transport Reviews 24, no. 5 (2004): 533-557.

Vlahogianni, Eleni I., Matthew G. Karlaftis, and John C. Golias. 2014. "Short-term traffic forecasting: where we are and where we're going." Transportation Research Part C: Emerging Technologies 43, (2014): 3-19.

Vlahogianni, Eleni I., Matthew G. Karlaftis, and John C. Golias. 2014. "Short-term traffic forecasting: where we are and where we're going." Transportation Research Part C: Emerging Technologies 43, (2014): 3-19.

Vojnikova, Dessislava. 2009. "Cluster analysis with SPSS: K-Means cluster analysis." http://evlm.stuba.sk/ partner2/STUDENTBOOK/English/SPSS_CA_2_EN.pdf.

Vyas, A. D., D. M. Patel, and K. M. Bertram. 2013. Potential for energy efficiency improvement beyond the light-duty-vehicle sector. DOE/GO-102013-3706, 2013.

Vyas, Anant, Dan Santini, and Larry Johnson. 2009. "Plug-in hybrid electric vehicles; potential for petroleum use reduction: issues involved in developing reliable estimates." In 88th Annual Meeting of the Transportation Research Board.Washington, D.C., 2009.

Wang, Rui, and Srdjan M. Lukic. 2011. "Review of driving conditions prediction and driving style recognition based control algorithms for hybrid electric vehicles." In 2011 IEEE Vehicle Power and Propulsion Conference. pp. 1-7. IEEE, 2011.

Wang, Rui, and Srdjan M. Lukic. 2011. "Review of driving conditions prediction and driving style recognition based control algorithms for hybrid electric vehicles." In 2011 IEEE Vehicle Power and Propulsion Conference. pp. 1-7. IEEE, 2011.

Williamson, Sheldon S., Sanjaka G. Wirasingha, and Ali Emadi. 2006. "Comparative investigation of series and parallel hybrid electric drive trains for heavy-duty transit 
bus applications." In 2006 IEEE Vehicle Power and Propulsion Conference. pp. 110, IEEE, 2006.

Wirasingha, Sanjaka G., and Ali Emadi. 2011. "Classification and review of control strategies for plug-in hybrid electric vehicles." IEEE Transactions on vehicular technology 60, no. 1 (2011): 111-122.

Xu, Ting, Xiaoduan Sun, Yan Wu, Yulong He, and Changrong Xie. 2009. "Artificial neural network and wavelet analysis application in speed forecast of Beijing urban freeway." In Intelligent Vehicles Symposium, 2009 IEEE. pp. 1004-1008. IEEE, 2009.

Xu, Ting, Xiaoduan Sun, Yan Wu, Yulong He, and Changrong Xie. 2009. "Artificial neural network and wavelet analysis application in speed forecast of Beijing urban freeway." In Intelligent Vehicles Symposium, 2009 IEEE. pp. 1004-1008. IEEE, 2009.

Ye, Qing, W. Y. Szeto, and S. C. Wong. 2012. "Short-term traffic speed forecasting based on data recorded at irregular intervals." IEEE Transactions on Intelligent Transportation Systems 13, no. 4 (2012): 1727-1737.

Zeng, Xiaohua. 2005. Performance and Fuel Economy Test Report of Hybrid Transit Bus. Jilin University.

Zhang, Bingzhan, Chris Chunting Mi, and Mengyang Zhang. 2011. "Charge-depleting control strategies and fuel optimization of blended-mode plug-in hybrid electric vehicles." IEEE Transactions on Vehicular Technology 60, no. 4 (2011): 15161525.

Zhang, Chen. 2010. Predictive energy management in connected vehicles: utilizing route information preview for energy saving. Clemson University. 


\section{Appendix}

Appendix A: Simulation Results between Rule-Based and DP over the WVU-CSI Cycle

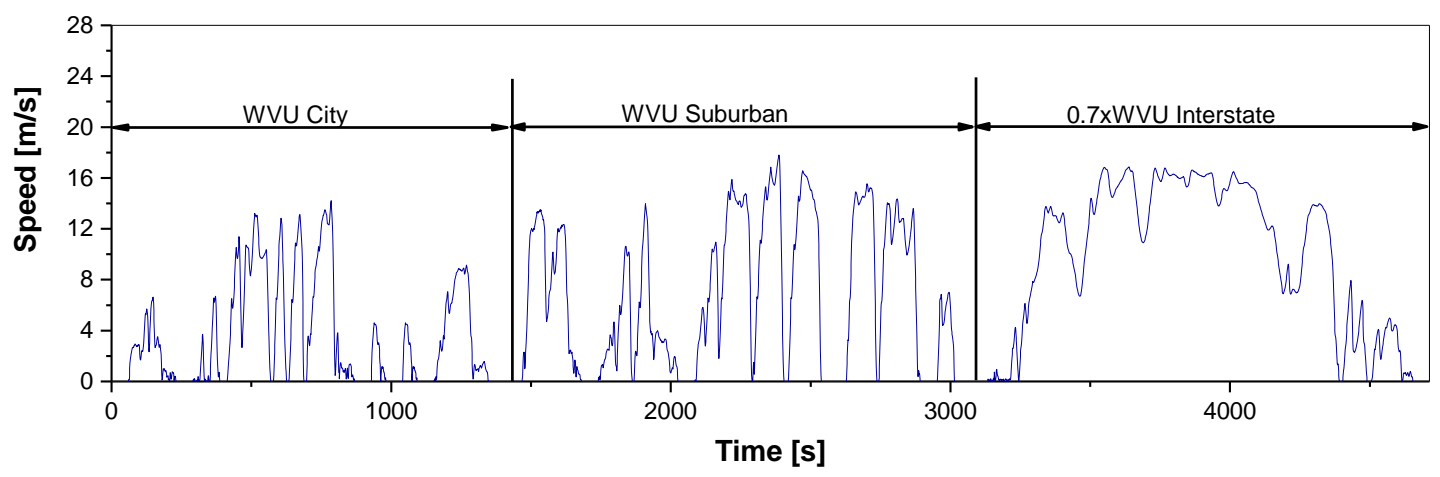

Figure A. 1 WVU-CSI cycle

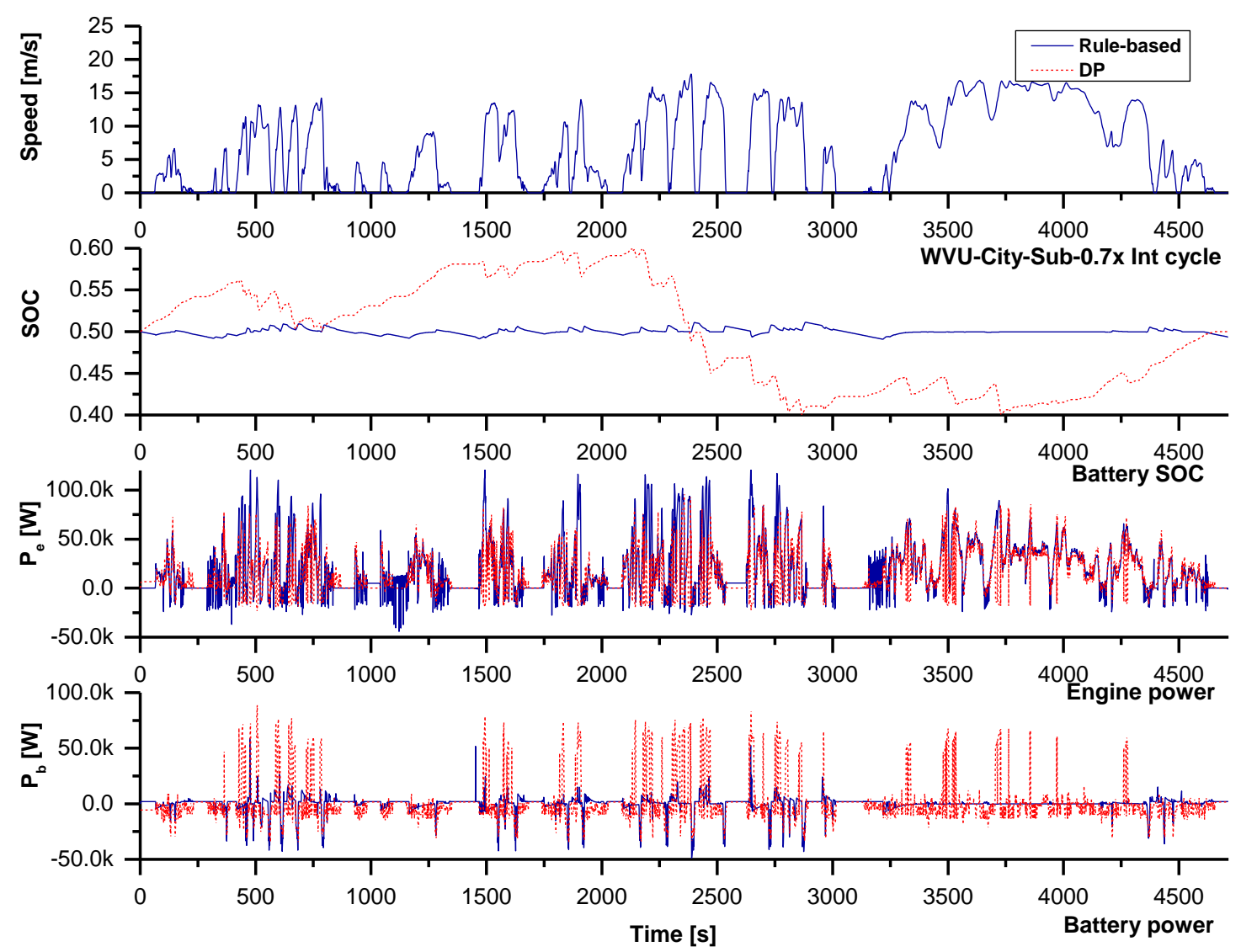

Figure A. 2 The operation parameters obtained with rule-based and DP $(\alpha=\beta=\gamma=0)$ in the WVU-CSI cycle 


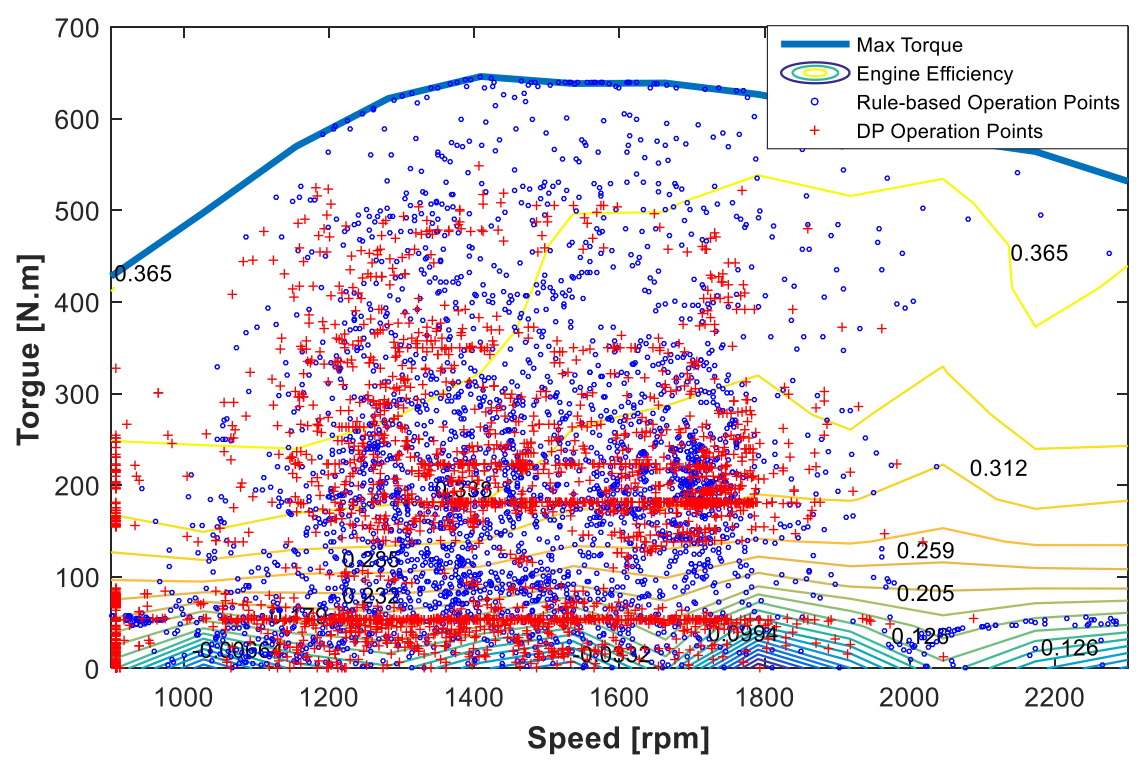

(a) Engine operation points distribution

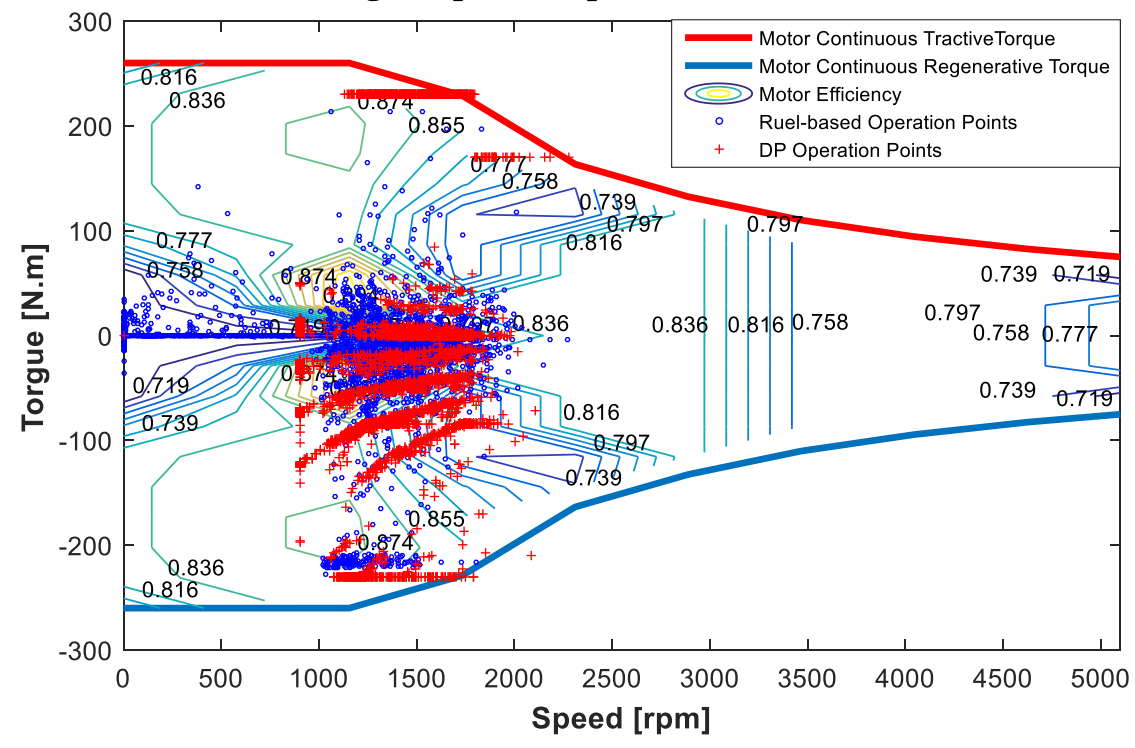

(a) Motor operation points distribution

Figure A. 3 Rule-based and DP $(\alpha=\beta=\gamma=0)$ power source operation points distribution in maps (WVU-CSI cycle) 

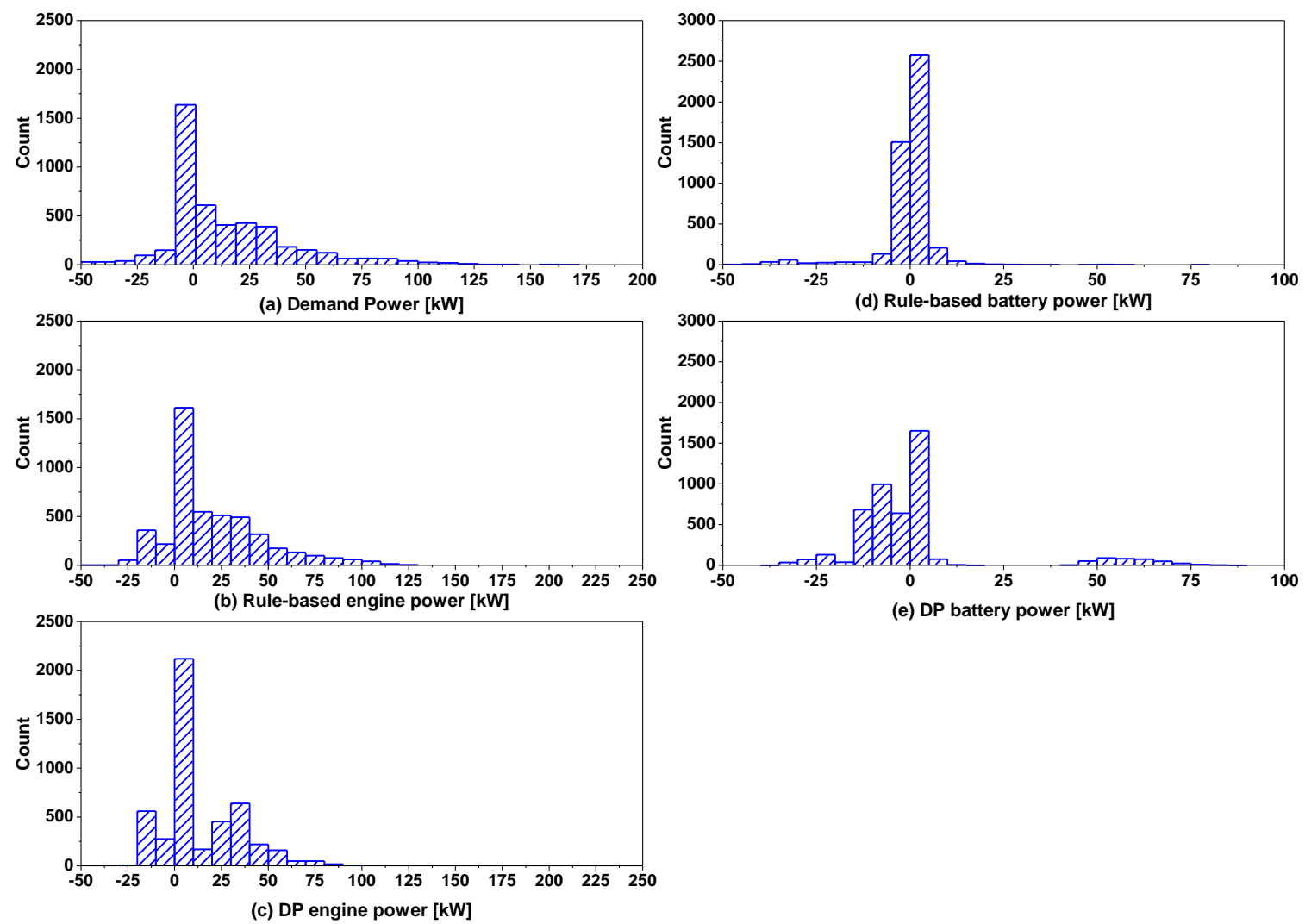

(e) DP battery power $[\mathrm{kW}]$

Figure A. 4 Distribution of the (a) demand power, (b) rule-based engine power, (c) DP engine power, (d) rule-based battery power and (e) DP battery power during the WVU-CSI cycle

Table A. 1 DP $(\alpha=\beta=\gamma=0)$ and rule-based simulation results for the WVU-CSI cycle

\begin{tabular}{|c|c|c|c|}
\hline & FE $(\mathbf{m p g})$ & FC $($ gram/mi) & NOx (gram/mi) \\
\hline Rule-based & 8.74 & 354.71 & 12.61 \\
\hline DP $(\boldsymbol{\alpha}=\mathbf{0}, \boldsymbol{\beta}=\mathbf{0}, \boldsymbol{\gamma}=\mathbf{0})$ & 11.78 & 267.42 & 10.41 \\
\hline
\end{tabular}

Note: $\alpha, \beta, \gamma$ is the weighting factors of battery current, engine turn on/off, and NOx emissions 
Appendix B: The Comparison between Optimal ECMS and DP Simulation Results over the WVU-CSI Cycle

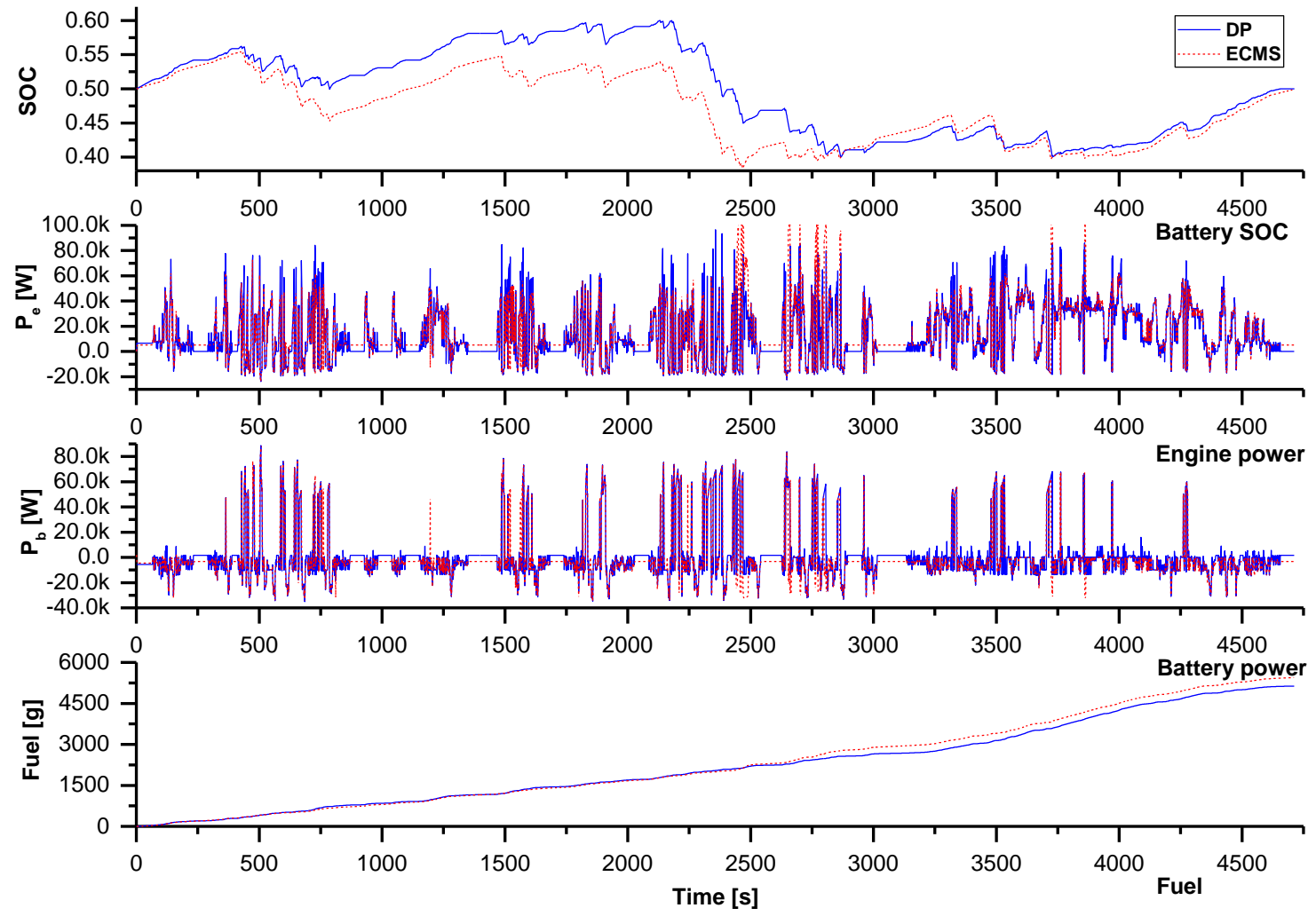

Figure B. 1 The operation parameters obtained with ECMS $\left(\lambda_{\text {opt }}=2.655\right)$ and DP $(\alpha=\beta=\gamma=0)$ (WVU-CSI cycle) 


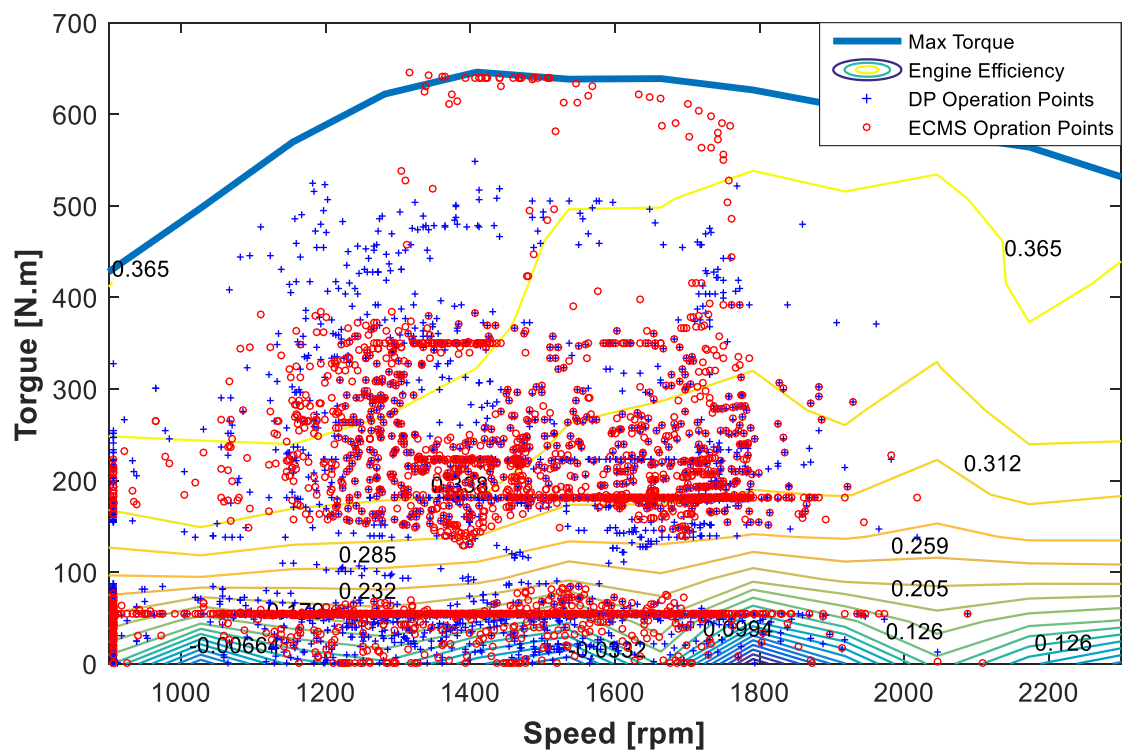

(a) Engine operation points distribution

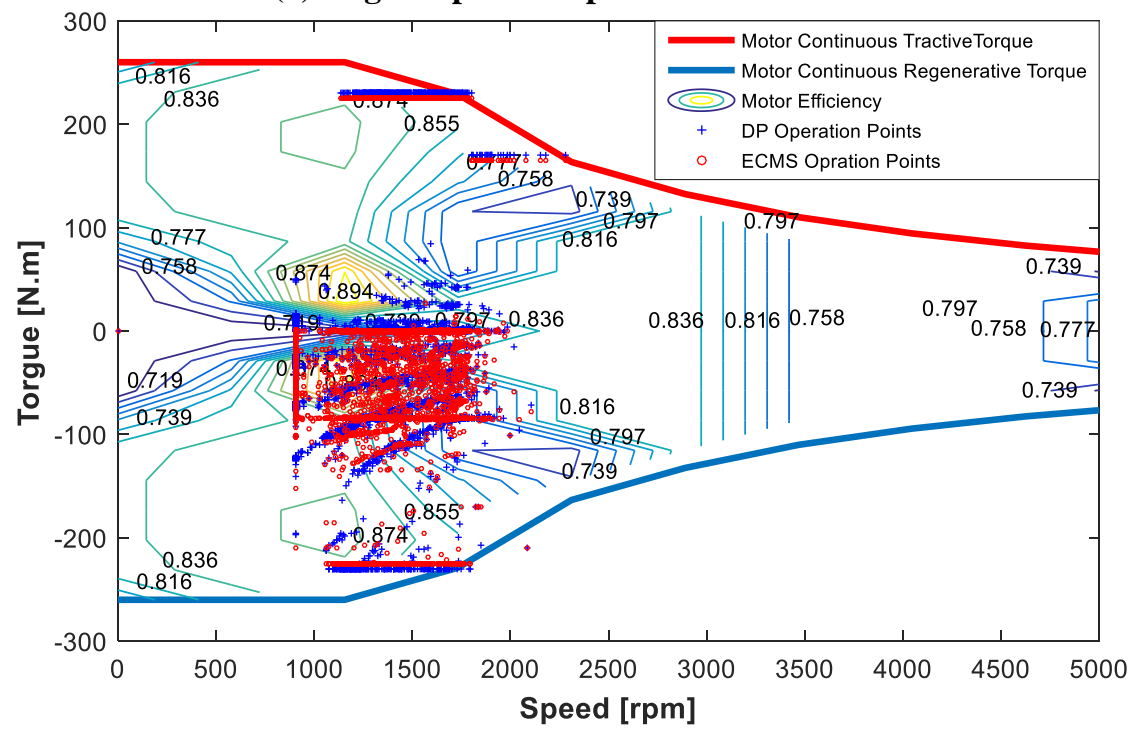

(b) Motor operation points distribution

Figure B. 2 Optimal ECMS $\left(\lambda_{\text {opt }}=2.655\right)$ and DP $(\alpha=\beta=\gamma=0)$ power source operation points distribution in maps (WVU-CSI cycle)

Table B. 1 DP $(\alpha=\beta=\gamma=0)$ and Optimal ECMS $(\lambda 0 p t=2.655)$ simulation results for the WVUCSI cycle

\begin{tabular}{|c|c|c|c|c|}
\hline & FE $(\mathbf{m p g})$ & $\Delta$ SOC & $F E_{\text {corr }}(\mathbf{m p g})$ & Gap to DP \\
\hline Optimal ECMS $\left(\lambda=\lambda_{\text {opt }}=\mathbf{2 . 6 5 5}\right)$ & 11.10 & -0.09 & 11.09 & $5.96 \%$ \\
\hline DP $(\boldsymbol{\alpha}=\mathbf{0}, \boldsymbol{\beta}=\mathbf{0}, \boldsymbol{\gamma}=\mathbf{0})$ & $\mathbf{1 1 . 7 8}$ & $\mathbf{0 . 0 0}$ & $\mathbf{1 1 . 7 8}$ & - \\
\hline
\end{tabular}


Appendix C: The Extracted $\lambda$ Values Obtained by ECMSwDP and the Comparison of ECMS with or without a Penalty $\left(\lambda=\lambda_{0}=\lambda_{\text {ave }}=2.115\right)$ and DP Simulation Results over the WVU-CSI Cycle

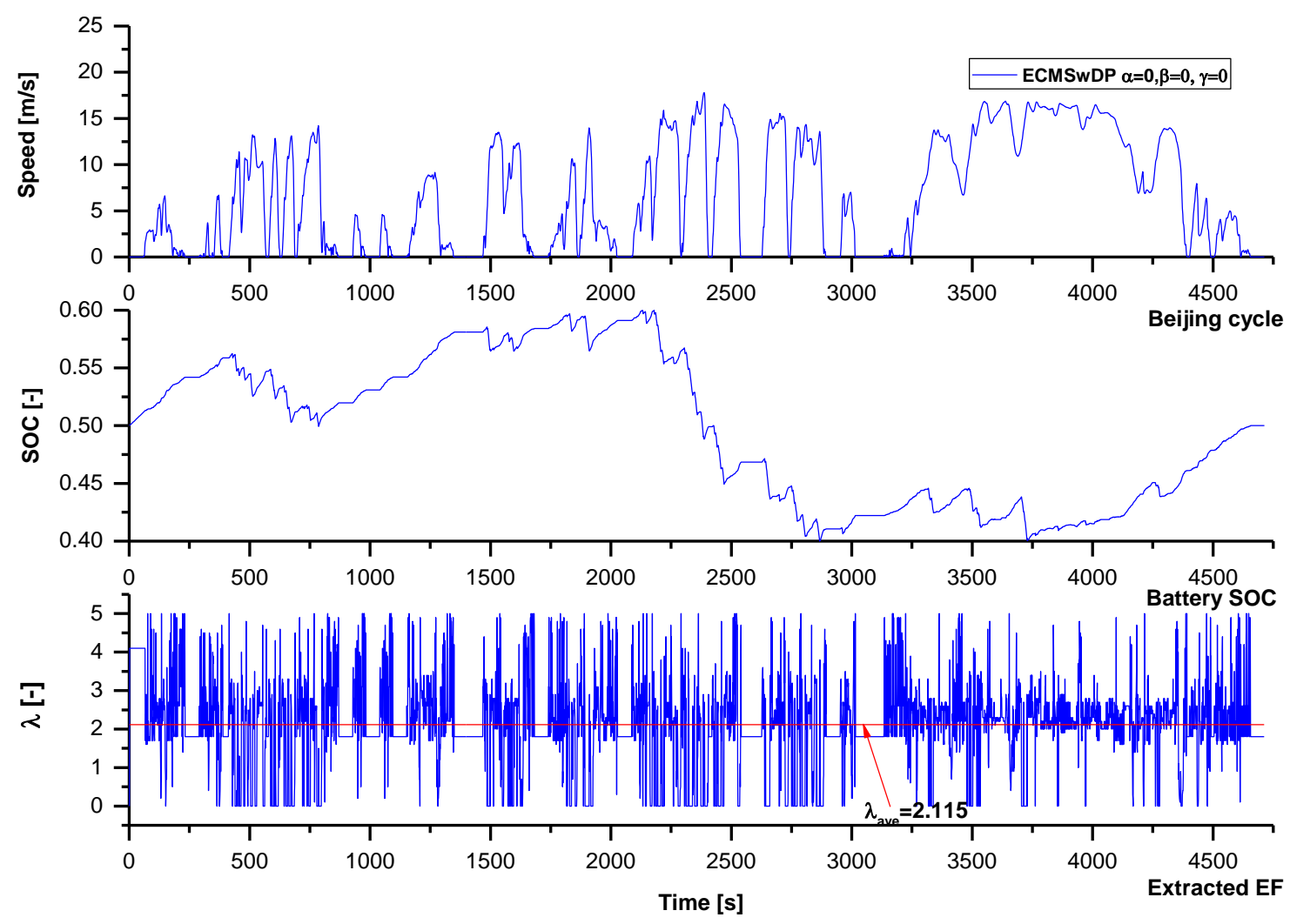

Figure $\mathrm{C}$. 1 The $\lambda$ values extracted using ECMSwDP over the WVU-CSI cycle 

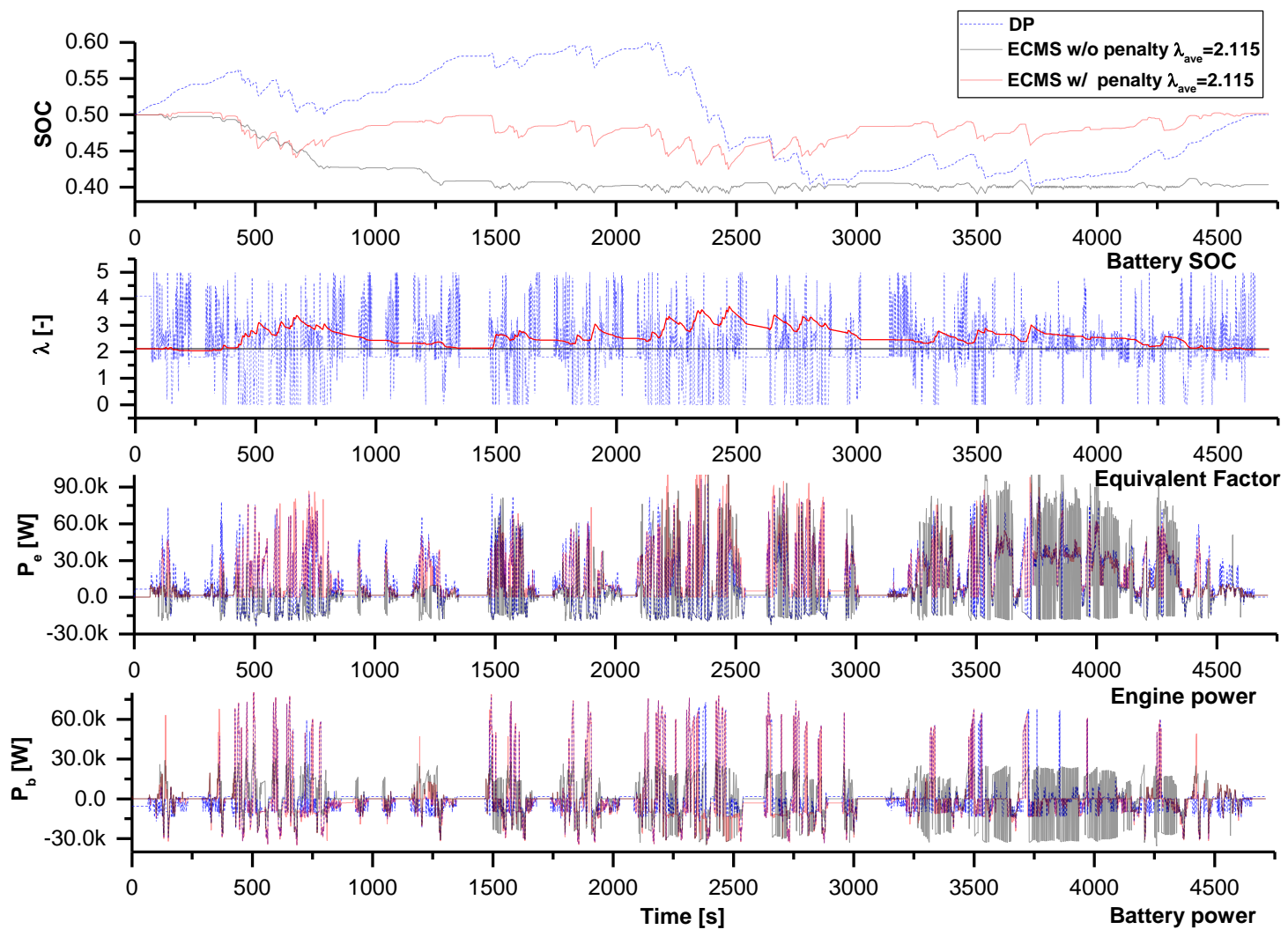

Figure C. 2 The operation parameters obtained using ECMS with or without penalty function $\left(\lambda_{\mathrm{ave}}=2.115\right)$ and $\mathrm{ECMSwDP}(\alpha=\beta=\gamma=0)$ simulation results (WVU-CSI cycle) 
Appendix D: The Obtained $\lambda$ Maps and the Simulation Results of A-ECMS Based on $\lambda$ maps as a Function of SOC, v, $\mathrm{P}_{\text {req }}$ and an Obtained by ECMSwDP over the WVU-CSI Cycle

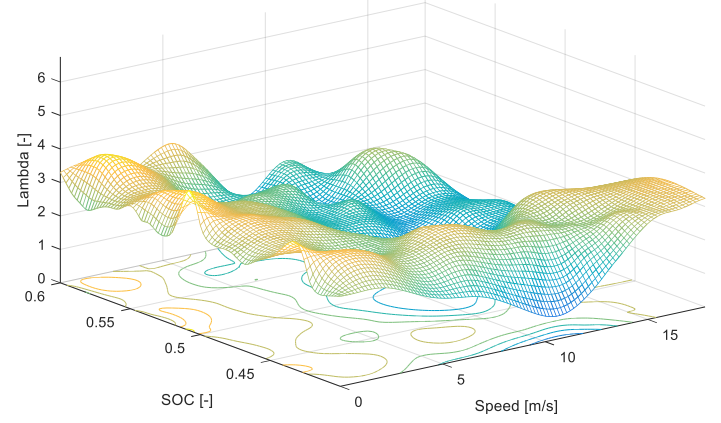

A. $\lambda(S O C, v)$

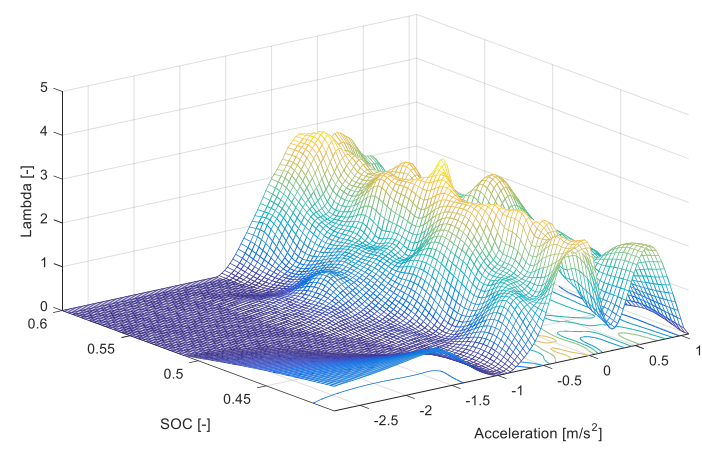

C. $\lambda(S O C, a)$

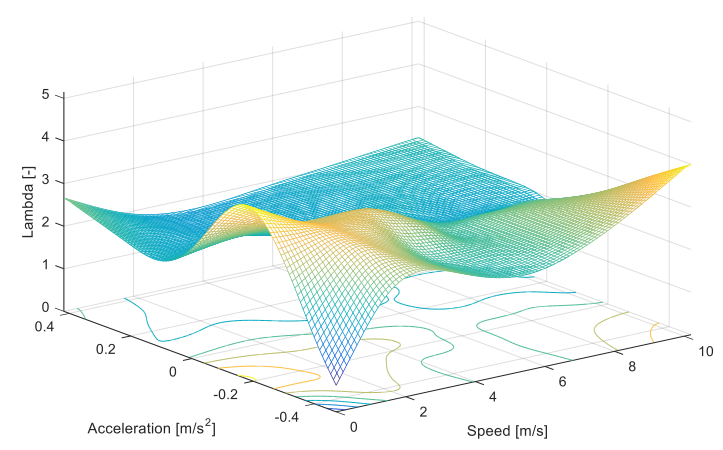

E. $\lambda(a, v)$

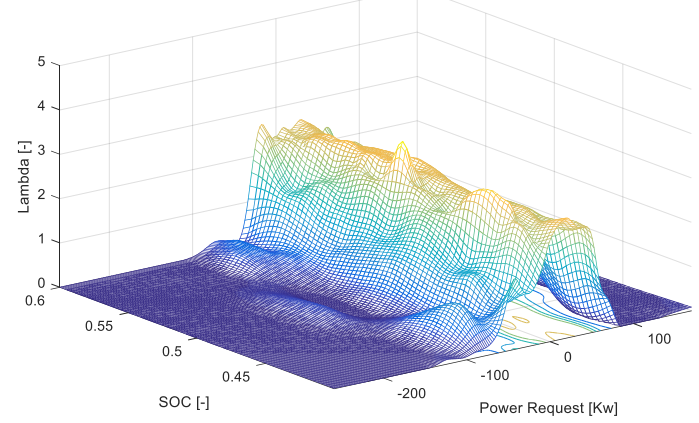

B. $\lambda\left(S O C, P_{\text {req }}\right)$

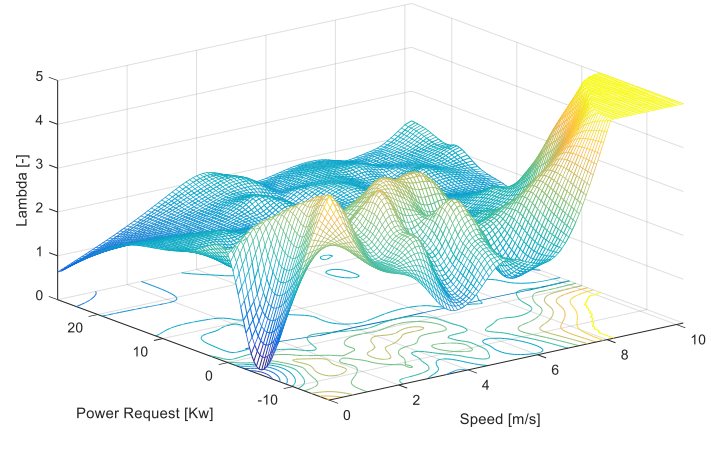

D. $\lambda\left(P_{\text {req }}, v\right)$

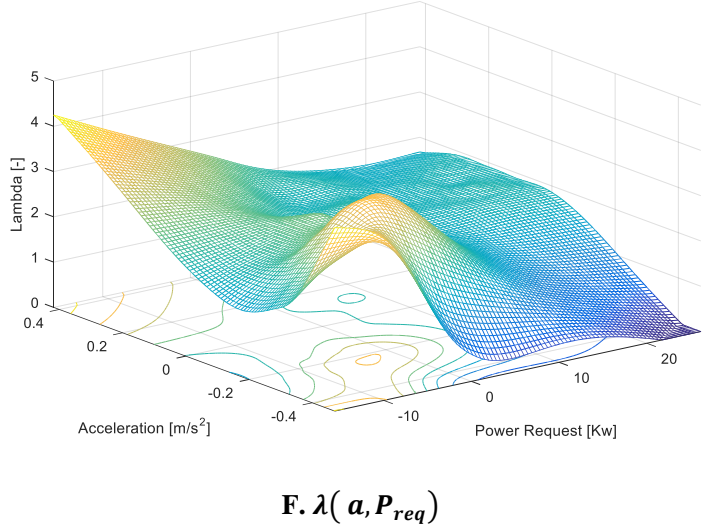

Figure D. 1 The $\lambda$ maps as a function of $S O C, v, P_{r e q}$ and $a$ obtained by ECMSwDP over the WVU-CSI cycle 

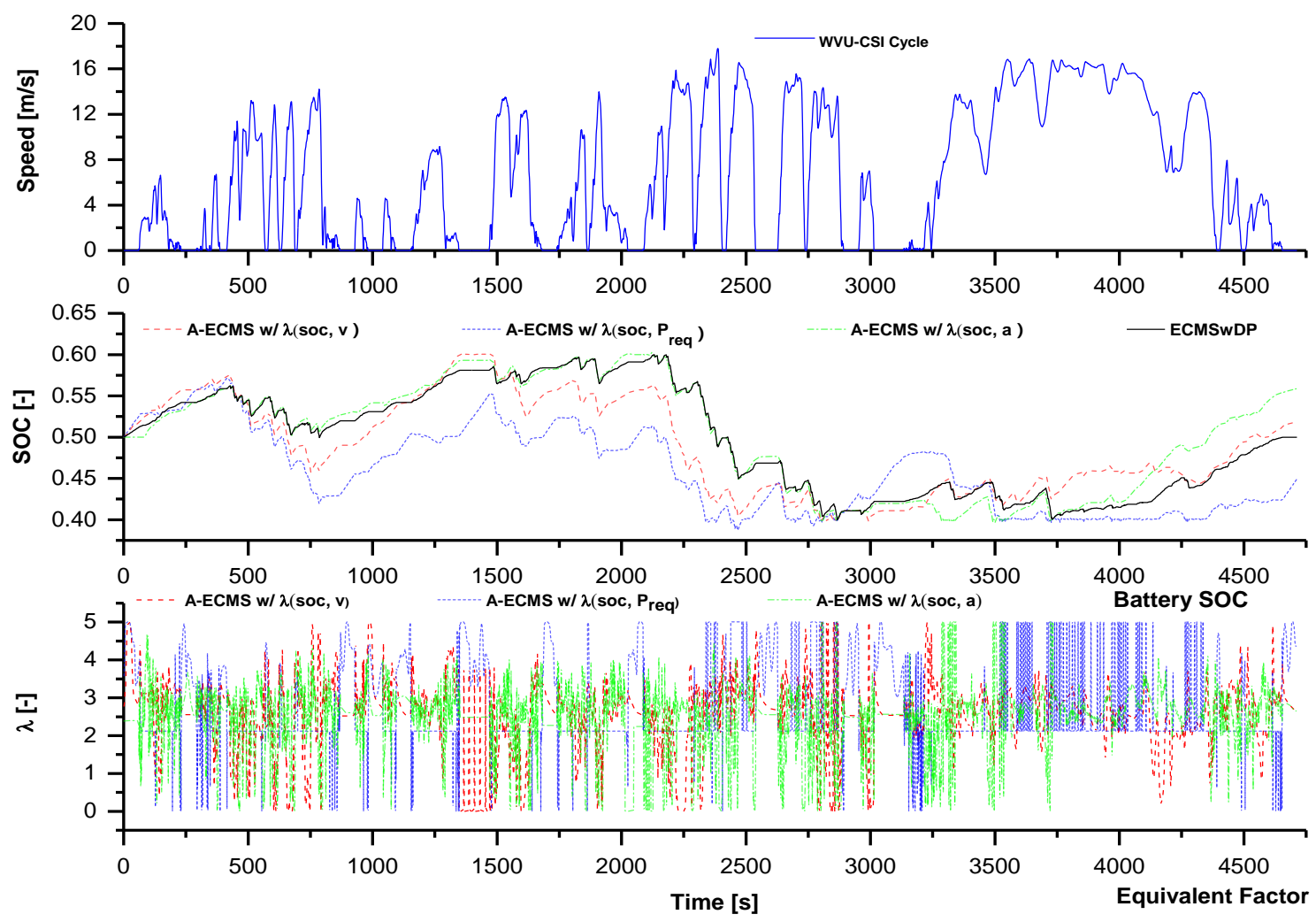

Figure D. 2 The operation parameters obtained with The operation parameters obtained with The operation parameters obtained with A-ECMS based on $\lambda$ maps as a function of $S O C, v, P_{r e q}$ and $a$ obtained by ECMSwDP and DP simulation results over the WVU-CSI cycle 

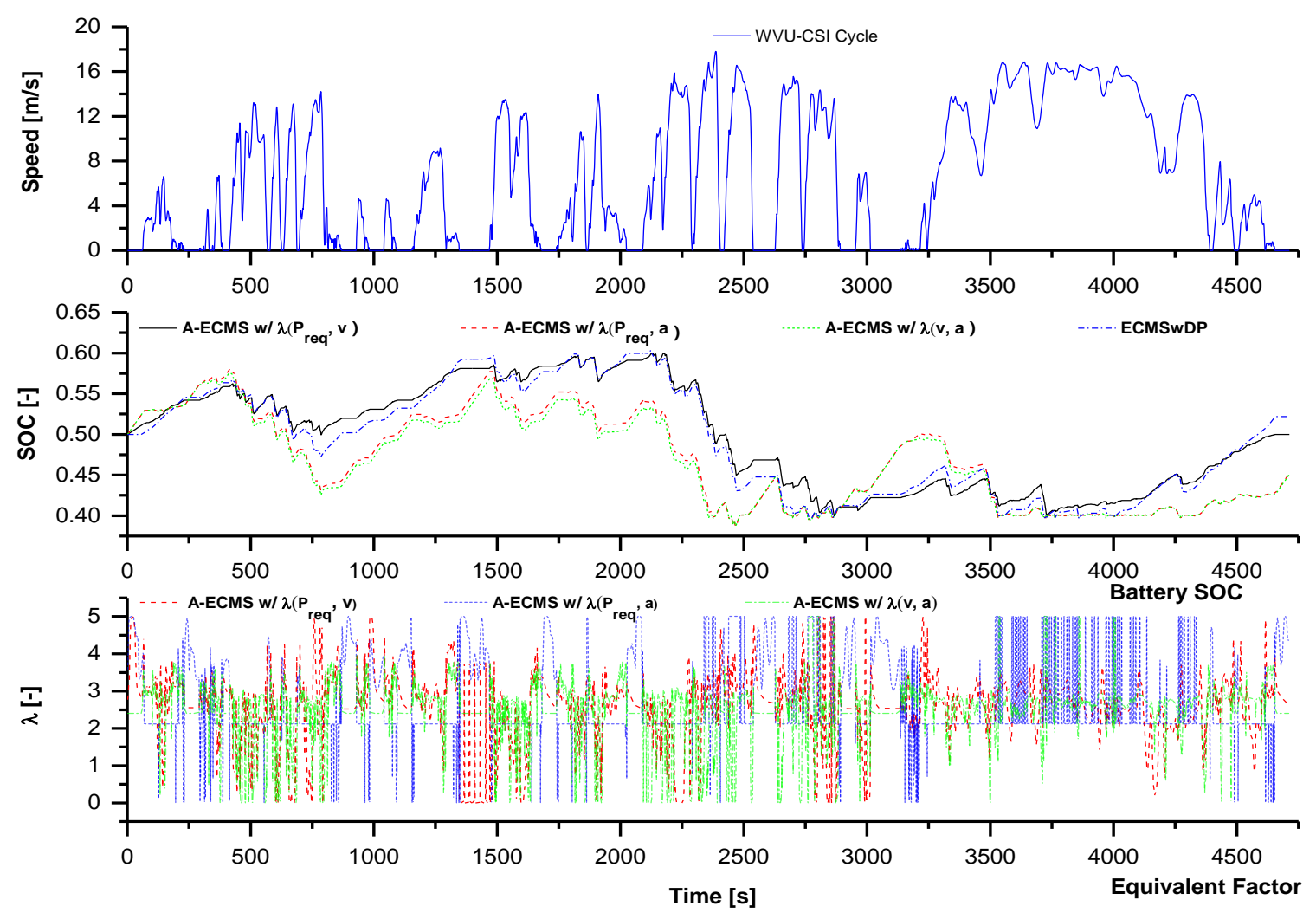

Figure D. 3 The comparison between A-ECMS based on $\lambda$ maps as function of $v, P_{r e q}$ and $a$ obtained by ECMSwDP and DP simulation results over the WVU-CSI cycle 
Appendix E: Simulation Results of A-ECMS Based on $\lambda$ Maps with Driving Pattern Identification over the WVU-CSI Cycle

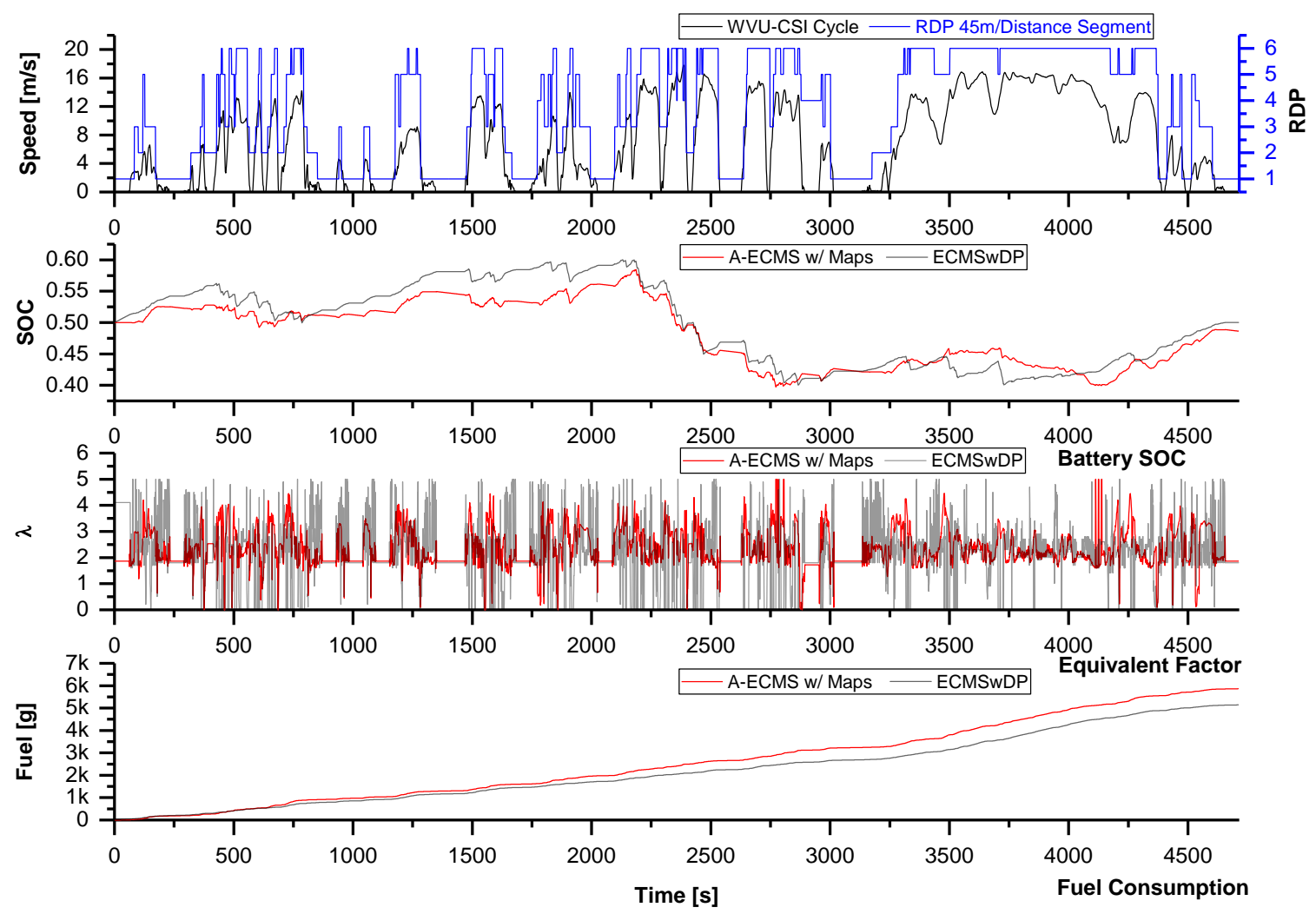

Figure E. 1 The comparison between A-ECMS based on $\lambda$ maps with driving pattern identification and ECMSwDP simulation results over the WVU-CSI cycle 
Appendix F: Simulation Results of A-ECMS with or without a Penalty based on the Average of the $\lambda$ Extracted with the Driving Pattern Identification over the WVU-CSI Cycle
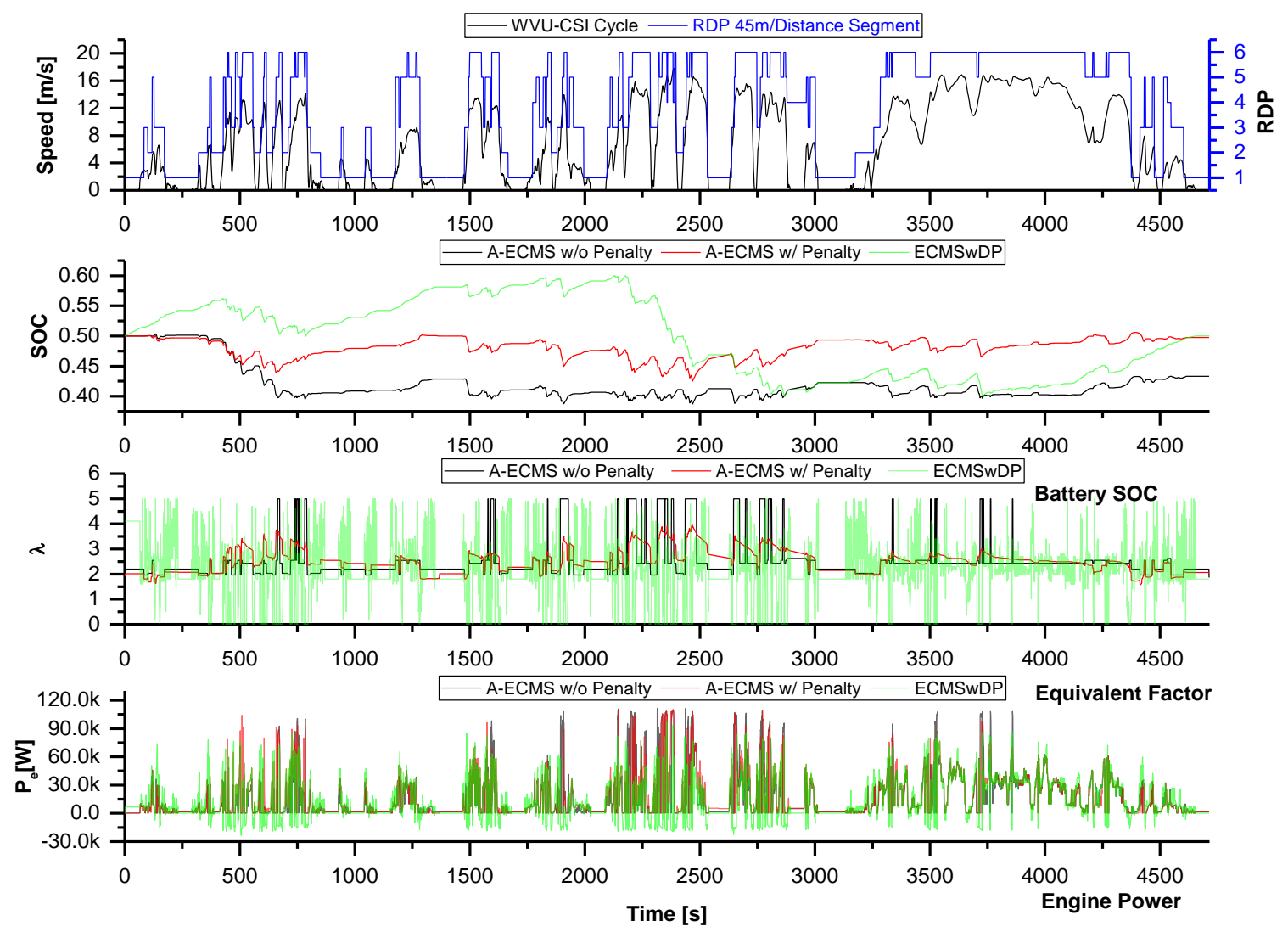

Figure F. 1 The comparison between A-ECMS with or without a penalty based on the average of the $\lambda$ extracted with the driving pattern identification and ECMSwDP simulation results over the WVU-CSI cycle 